\title{
Introduction of Aryl Fluorosulfates into the Realm of Catellani Reaction Substrates
}

\author{
Vidmantas Bieliūnas and Wim M. De Borggraeve* \\ Molecular Design and Synthesis, Department of Chemistry, KU Leuven, \\ Celestijnenlaan 200F, box 2404, 3001 Leuven, Belgium. \\ *E-mail: wim.deborggraeve@kuleuven.be
}

\section{Supporting Information}

1

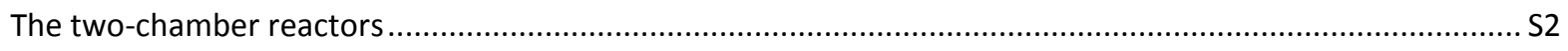

2 Optimization of reaction parameters for activated substrates ............................................................... S3

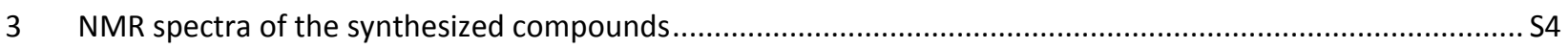

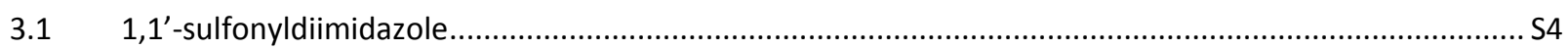

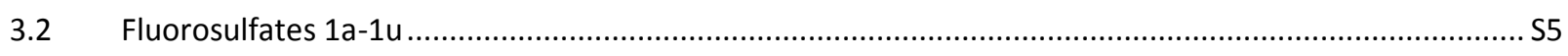

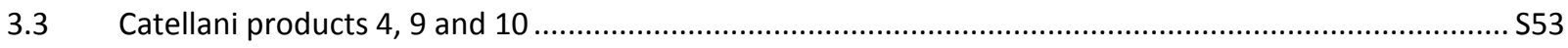




\section{The two-chamber reactors}

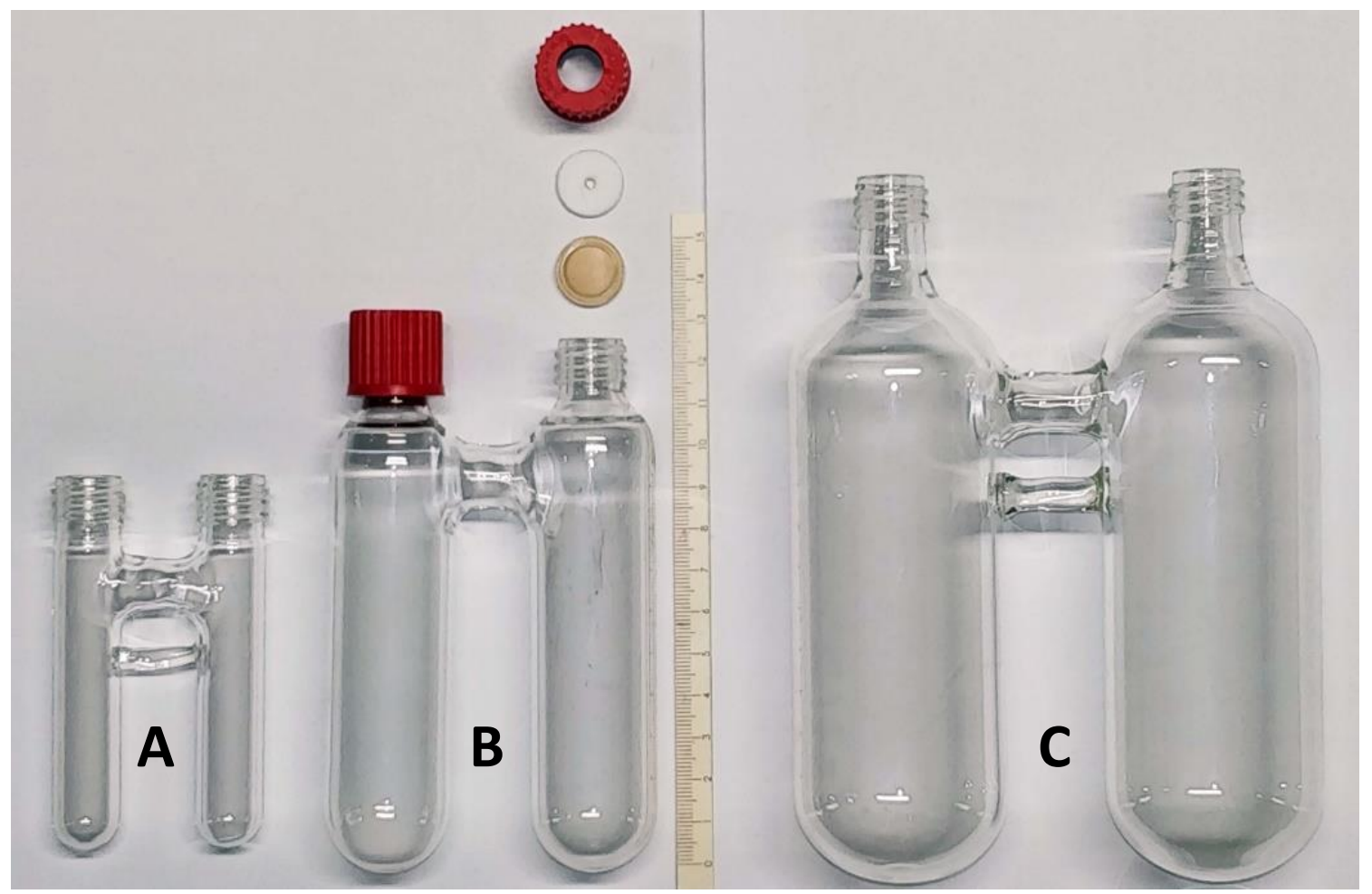

Figure S1-1. A small two chamber reactor $\left(\mathbf{A}, \mathrm{V}_{\text {total }}=10-12 \mathrm{~mL}\right)$, an intermediate-size two-chamber reactor $\left(\mathbf{B}, \mathrm{V}_{\text {total }}=100-110\right.$ $\mathrm{mL}$ ) with GL14 hollow cap, PTFE disk and septum assembly, and a large two-chamber reactor $\left(C, V_{\text {total }}=400-425 \mathrm{~mL}\right)$. Invented by the Skrydstrup group. ${ }^{2}$ 


\section{Optimization of reaction parameters for activated substrates}

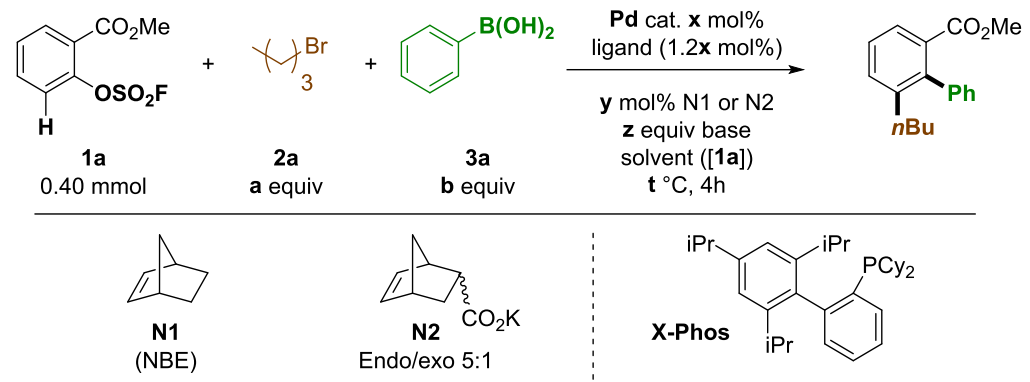

Table S1. Optimization of reaction conditions. Groups of directly comparable single-variable changes are shaded accordingly.

\begin{tabular}{|c|c|c|c|c|c|c|c|c|c|c|c|}
\hline Entry & a & b & Pd cat. & $x$ & Ligand & $y$ & Base (z) & Solvent & [1a], M & $t,{ }^{\circ} \mathrm{C}$ & Yield \\
\hline 1 & 2.00 & 1.20 & $\mathrm{Pd}(\mathrm{OAc})_{2}$ & 5 & - & 100 (N1) & $\mathrm{K}_{2} \mathrm{CO}_{3}(6.0)$ & DMF & 0.20 & 100 & $35 \%$ \\
\hline 2 & 2.00 & 1.20 & $\mathrm{Pd}(\mathrm{OAc})_{2}$ & 5 & - & 100 (N1) & $\mathrm{K}_{2} \mathrm{CO}_{3}(6.0)$ & DMF & 0.20 & 90 & $46 \%$ \\
\hline 3 & 2.00 & 1.20 & $\mathrm{Pd}(\mathrm{OAc})_{2}$ & 5 & - & 100 (N1) & $\mathrm{K}_{2} \mathrm{CO}_{3}(6.0)$ & DMF & 0.20 & 80 & $65 \%$ \\
\hline 4 & 3.00 & 1.20 & $\mathrm{Pd}(\mathrm{OAC})_{2}$ & 5 & - & 100 (N1) & $\mathrm{K}_{2} \mathrm{CO}_{3}(6.0)$ & DMF & 0.20 & 80 & $57 \%$ \\
\hline 5 & 1.50 & 1.20 & $\mathrm{Pd}(\mathrm{OAc})_{2}$ & 5 & - & 100 (N1) & $\mathrm{K}_{2} \mathrm{CO}_{3}(6.0)$ & DMF & 0.20 & 80 & $69 \%$ \\
\hline 6 & 1.50 & 1.20 & $\mathrm{Pd}(\mathrm{OAC})_{2}$ & 5 & - & 100 (N1) & $\mathrm{K}_{2} \mathrm{CO}_{3}(6.0)$ & DMF & 0.20 & 70 & $74 \%$ \\
\hline 7 & 1.50 & 1.20 & $\mathrm{Pd}(\mathrm{OAc})_{2}$ & 2 & - & 100 (N1) & $\mathrm{K}_{2} \mathrm{CO}_{3}(6.0)$ & DMF & 0.20 & 70 & $59 \%$ \\
\hline 8 & 1.50 & 1.20 & $\mathrm{Pd}(\mathrm{OAc})_{2}$ & 5 & - & 100 (N2) & $\mathrm{K}_{2} \mathrm{CO}_{3}(5.0)$ & DMF & 0.20 & 70 & $49 \%^{a}$ \\
\hline 9 & 1.50 & 1.20 & $\mathrm{Pd}(\mathrm{OAc})_{2}$ & 5 & - & 100 (N1) & $\mathrm{K}_{2} \mathrm{CO}_{3}(6.0)$ & DMAc & 0.20 & 70 & $65 \%$ \\
\hline 10 & 1.50 & 1.20 & $\mathrm{Pd}(\mathrm{OAc})_{2}$ & 5 & - & 100 (N1) & $\mathrm{K}_{2} \mathrm{CO}_{3}(6.0)$ & NMP & 0.20 & 70 & $60 \%$ \\
\hline 11 & 1.50 & 1.20 & $\mathrm{Pd}(\mathrm{OAc})_{2}$ & 5 & - & 100 (N1) & $\mathrm{K}_{2} \mathrm{CO}_{3}(6.0)$ & $\mathrm{MeCN}$ & 0.20 & 70 & $4 \%$ \\
\hline 12 & 1.50 & 1.20 & $\mathrm{Pd}(\mathrm{OAc})_{2}$ & 5 & - & 100 (N1) & $\mathrm{K}_{2} \mathrm{CO}_{3}(6.0)$ & $\mathrm{GVL}^{\mathrm{b}}$ & 0.20 & 70 & $0 \%$ \\
\hline 13 & 1.50 & 1.20 & $\mathrm{Pd}(\mathrm{OAc})_{2}$ & 5 & - & 100 (N1) & $\mathrm{K}_{2} \mathrm{CO}_{3}(6.0)$ & DMF & 0.20 & 60 & $61 \%^{c}$ \\
\hline 14 & 1.50 & 1.20 & $\mathrm{Pd}(\mathrm{OAc})_{2}$ & 5 & - & 100 (N1) & $\mathrm{K}_{2} \mathrm{CO}_{3}(6.0)$ & DMF & 0.20 & 50 & $10 \%^{c}$ \\
\hline 15 & 1.50 & 1.20 & $\mathrm{Pd}(\mathrm{OAc})_{2}$ & 5 & - & 100 (N1) & $\mathrm{Cs}_{2} \mathrm{CO}_{3}(6.0)$ & DMF & 0.20 & 70 & $68 \%$ \\
\hline 16 & 1.50 & 1.20 & $\mathrm{Pd}(\mathrm{OAc})_{2}$ & 5 & - & 100 (N1) & KOAC (6.0) & DMF & 0.20 & 70 & $26 \%$ \\
\hline 17 & 1.50 & 1.20 & $\mathrm{Pd}(\mathrm{OAc})_{2}$ & 5 & - & 100 (N1) & TEA (6.0) & DMF & 0.20 & 70 & $0 \%$ \\
\hline 18 & 1.50 & 1.20 & $\mathrm{Pd}(\mathrm{OAc})_{2}$ & 5 & - & 100 (N1) & $\mathrm{Bu}_{4} \mathrm{NOAC}(6.0)$ & DMF & 0.20 & 70 & $0 \%$ \\
\hline 19 & 1.50 & 1.20 & $\mathrm{Pd} / \mathrm{C}(10 \%)$ & 10 & - & 100 (N1) & $\mathrm{K}_{2} \mathrm{CO}_{3}(6.0)$ & DMF & 0.20 & 70 & $5 \%$ \\
\hline 20 & 1.50 & 1.20 & $\mathrm{Pd}\left(\mathrm{PPh}_{3}\right)_{4}$ & 5 & - & 100 (N1) & $\mathrm{K}_{2} \mathrm{CO}_{3}(6.0)$ & DMF & 0.20 & 70 & $0 \%^{d}$ \\
\hline 21 & 1.50 & 1.20 & $\mathrm{Pd}(\mathrm{OAc})_{2}$ & 5 & - & 100 (N1) & $\mathrm{K}_{2} \mathrm{CO}_{3}(6.0)$ & DMF & 0.50 & 70 & $64 \%$ \\
\hline 22 & 1.50 & 1.20 & $\mathrm{Pd}(\mathrm{OAc})_{2}$ & 5 & - & 50 (N1) & $\mathrm{K}_{2} \mathrm{CO}_{3}(6.0)$ & DMF & 0.50 & 70 & $78 \%$ \\
\hline 23 & 1.20 & 1.20 & $\mathrm{Pd}(\mathrm{OAc})_{2}$ & 5 & - & 50 (N1) & $\mathrm{K}_{2} \mathrm{CO}_{3}(6.0)$ & DMF & 0.50 & 70 & $81 \%$ \\
\hline 24 & 1.20 & 1.20 & $\mathrm{Pd}(\mathrm{OAc})_{2}$ & 5 & - & 50 (N1) & $\mathrm{K}_{2} \mathrm{CO}_{3}(6.0)$ & DMF & 1.00 & 70 & $54 \%$ \\
\hline 25 & 1.20 & 1.20 & $\mathrm{Pd}(\mathrm{OAc})_{2}$ & 5 & - & 25 (N1) & $\mathrm{K}_{2} \mathrm{CO}_{3}(6.0)$ & DMF & 0.50 & 70 & $76 \% \mathrm{e}^{\mathrm{e}}$ \\
\hline 26 & 1.10 & 1.20 & $\mathrm{Pd}(\mathrm{OAc})_{2}$ & 5 & - & 50 (N1) & $\mathrm{K}_{2} \mathrm{CO}_{3}(6.0)$ & DMF & 0.50 & 70 & $79 \%$ \\
\hline 27 & 1.20 & $1.25^{f}$ & $\mathrm{Pd}(\mathrm{OAc})_{2}$ & 5 & - & 50 (N1) & $\mathrm{K}_{2} \mathrm{CO}_{3}(6.0)$ & DMF & 0.50 & 70 & $\underline{82 \%}$ \\
\hline 28 & 1.20 & $1.25^{f}$ & $\mathrm{Pd}(\mathrm{OAc})_{2}$ & 7.5 & - & 50 (N1) & $\mathrm{K}_{2} \mathrm{CO}_{3}(6.0)$ & DMF & 0.50 & 70 & $83 \% g$ \\
\hline 29 & 1.20 & $1.25^{f}$ & $\mathrm{Pd}(\mathrm{OAC})_{2}$ & 5 & X-Phos & 50 (N1) & $\mathrm{K}_{2} \mathrm{CO}_{3}(6.0)$ & DMF & 0.50 & 70 & $74 \%$ \\
\hline
\end{tabular}

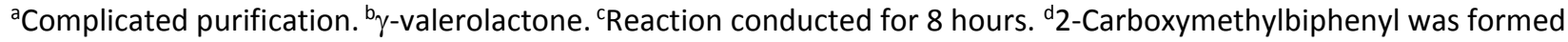

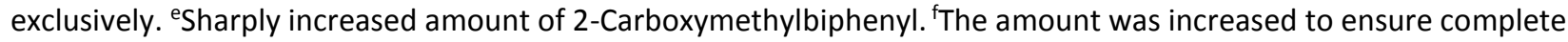
conversion over multiple repetitions. ${ }^{\mathrm{g} T h e}$ increase in yield was insufficient to justify the $50 \%$ increase in catalyst loading. 


\section{NMR spectra of the synthesized compounds}

\subsection{1,1'-sulfonyldiimidazole}
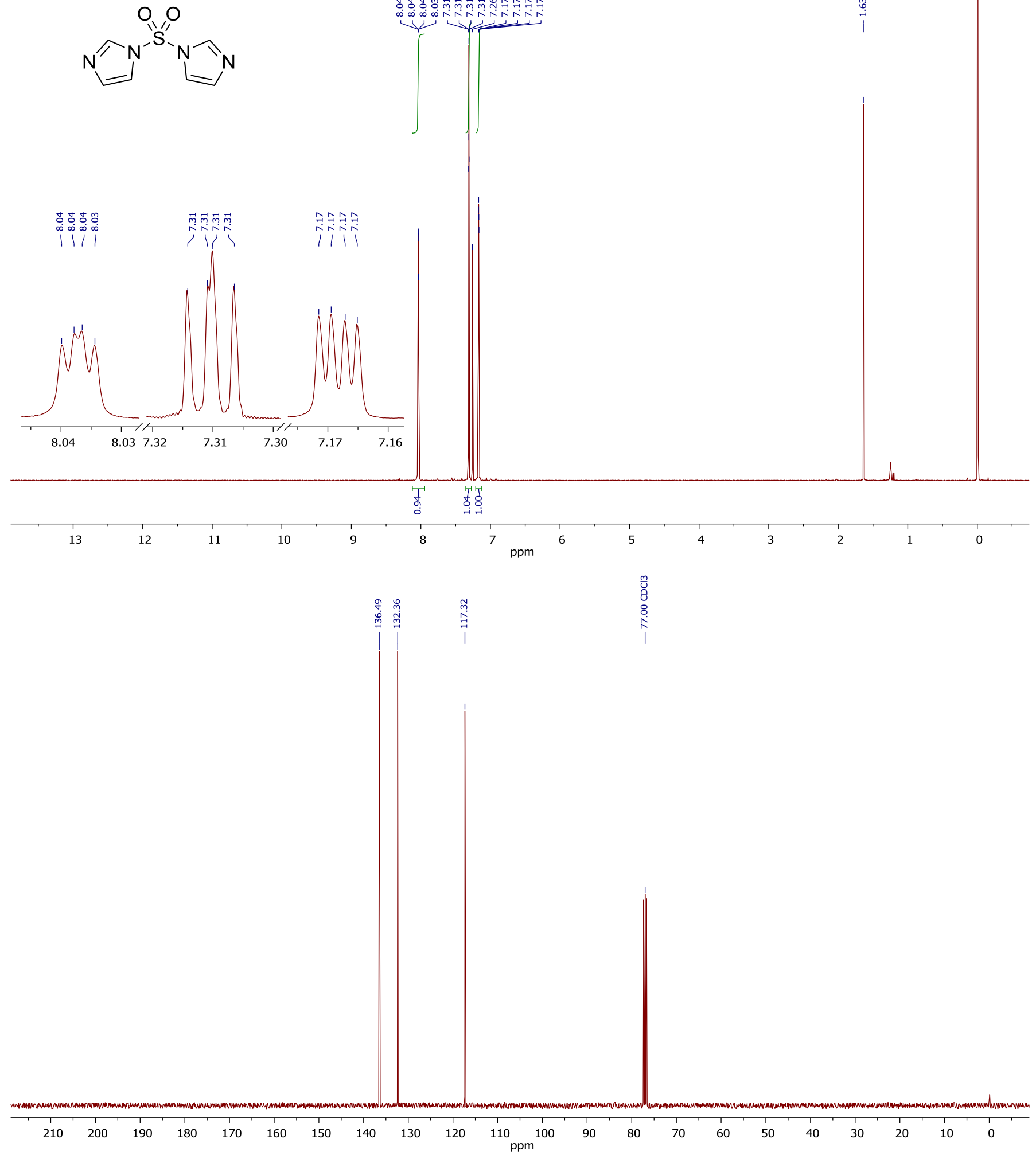


\subsection{Fluorosulfates 1a-1u}<smiles>COC(=O)c1ccccc1OS(=O)(=O)F</smiles>
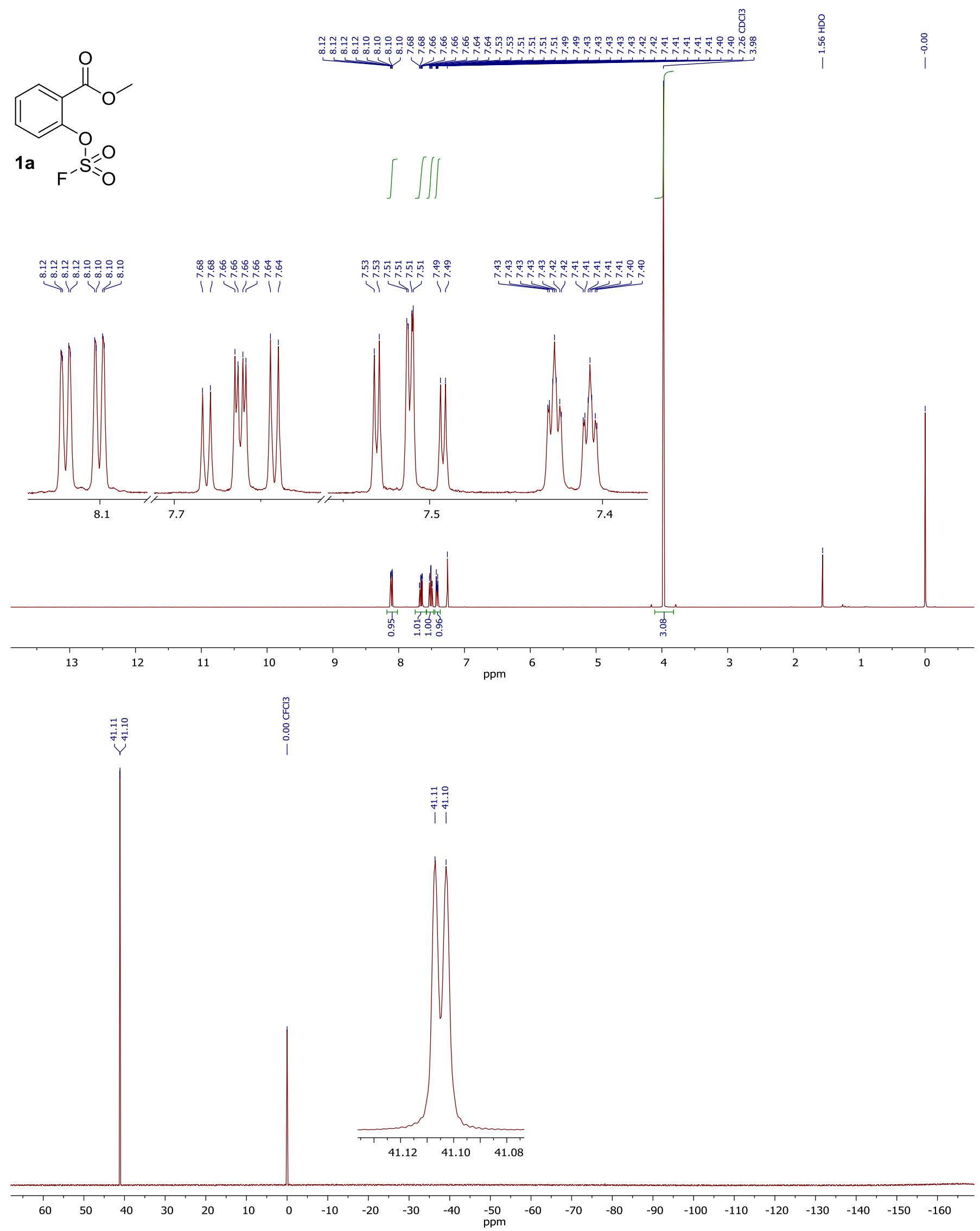

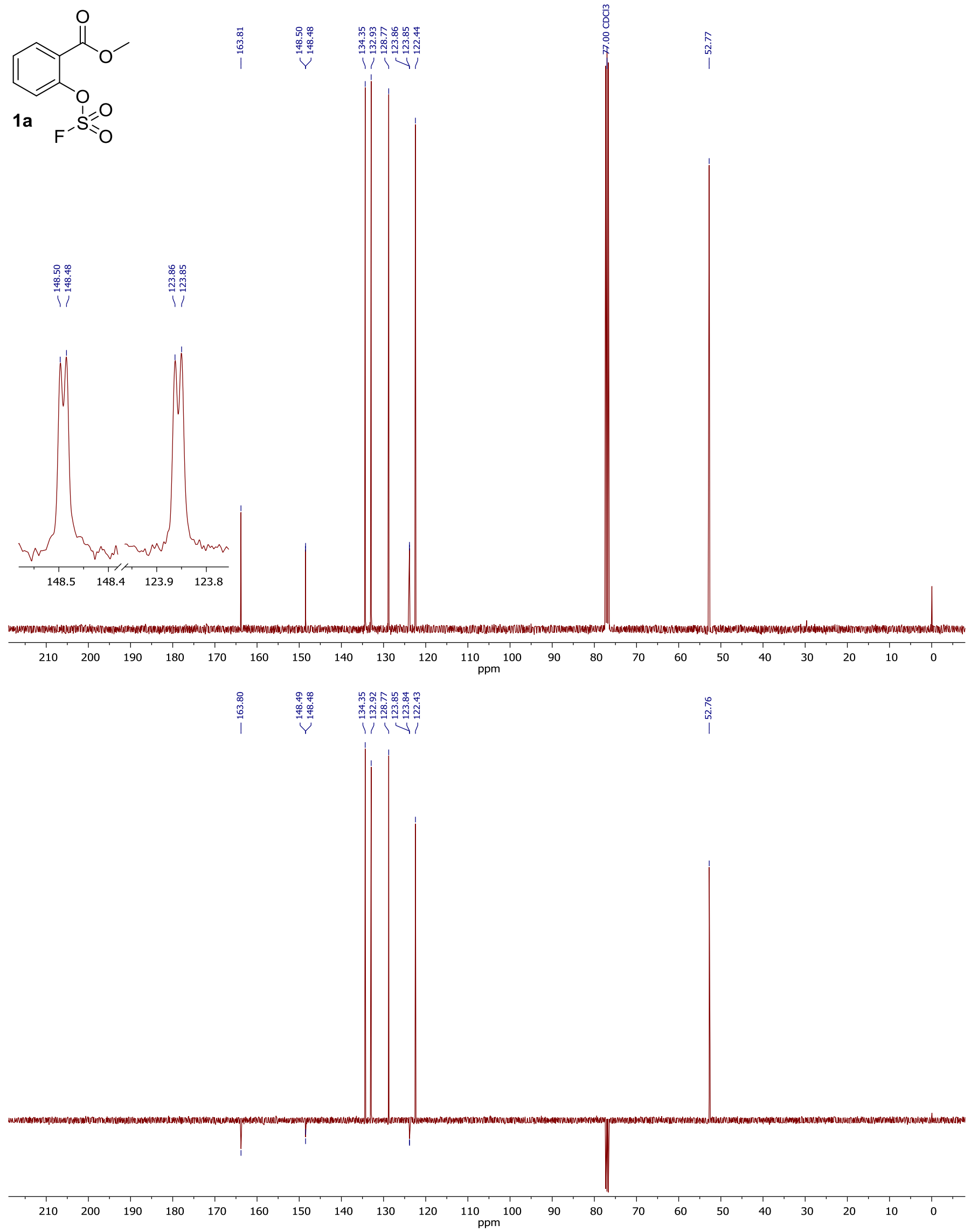

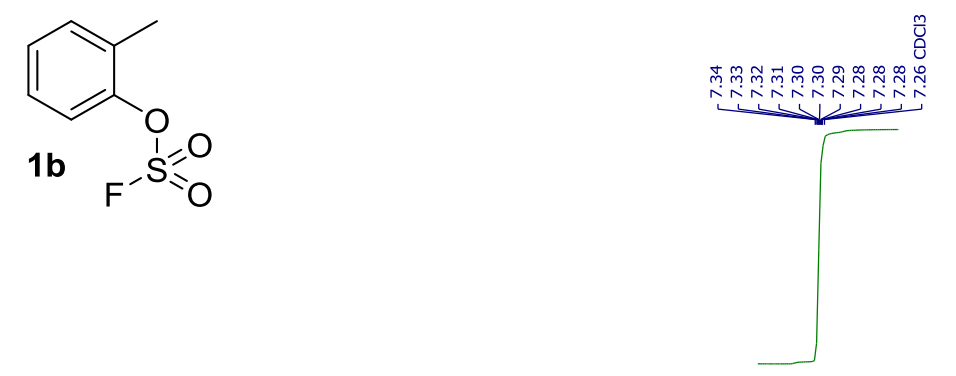

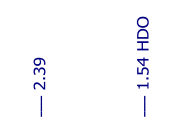
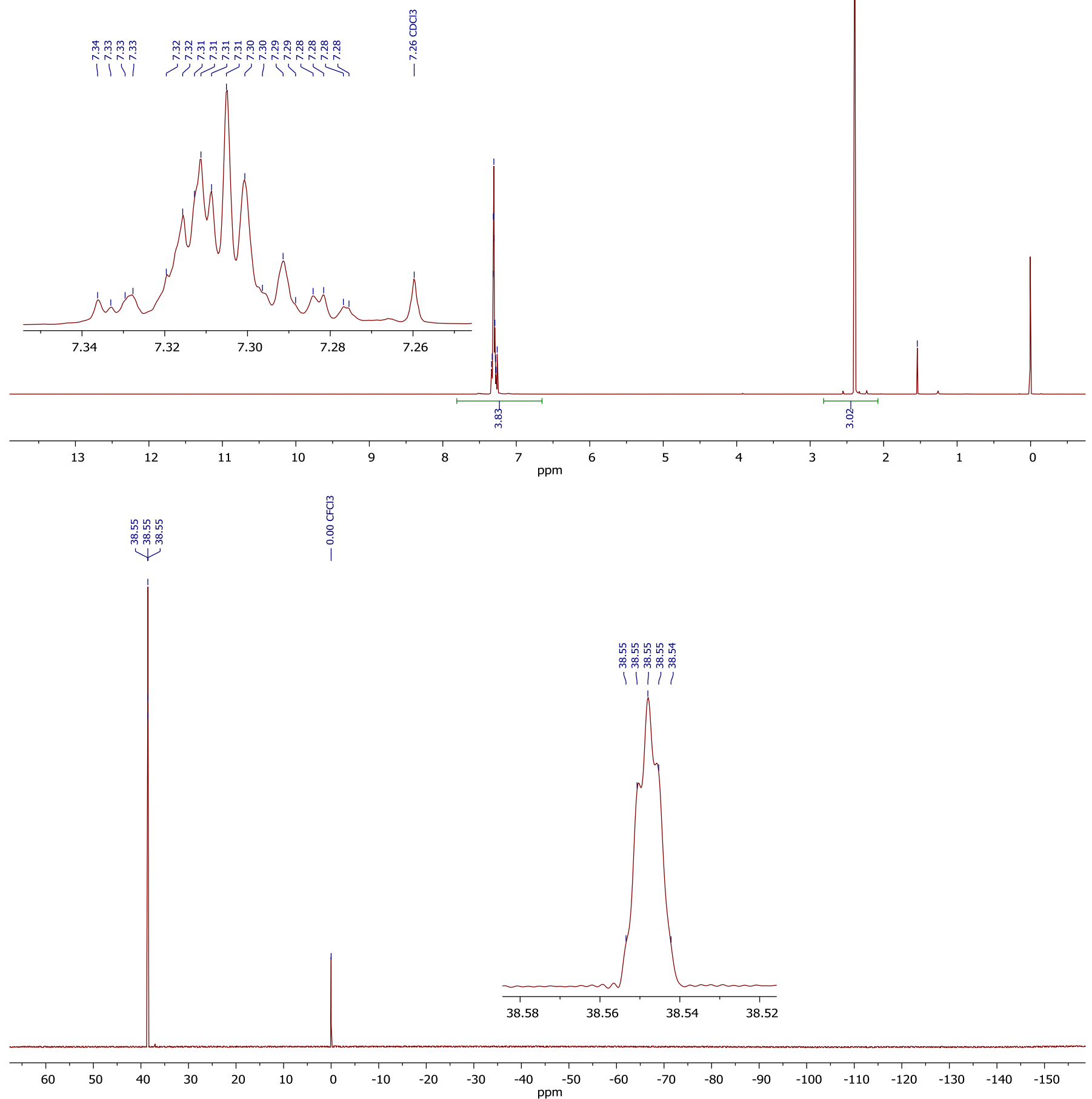


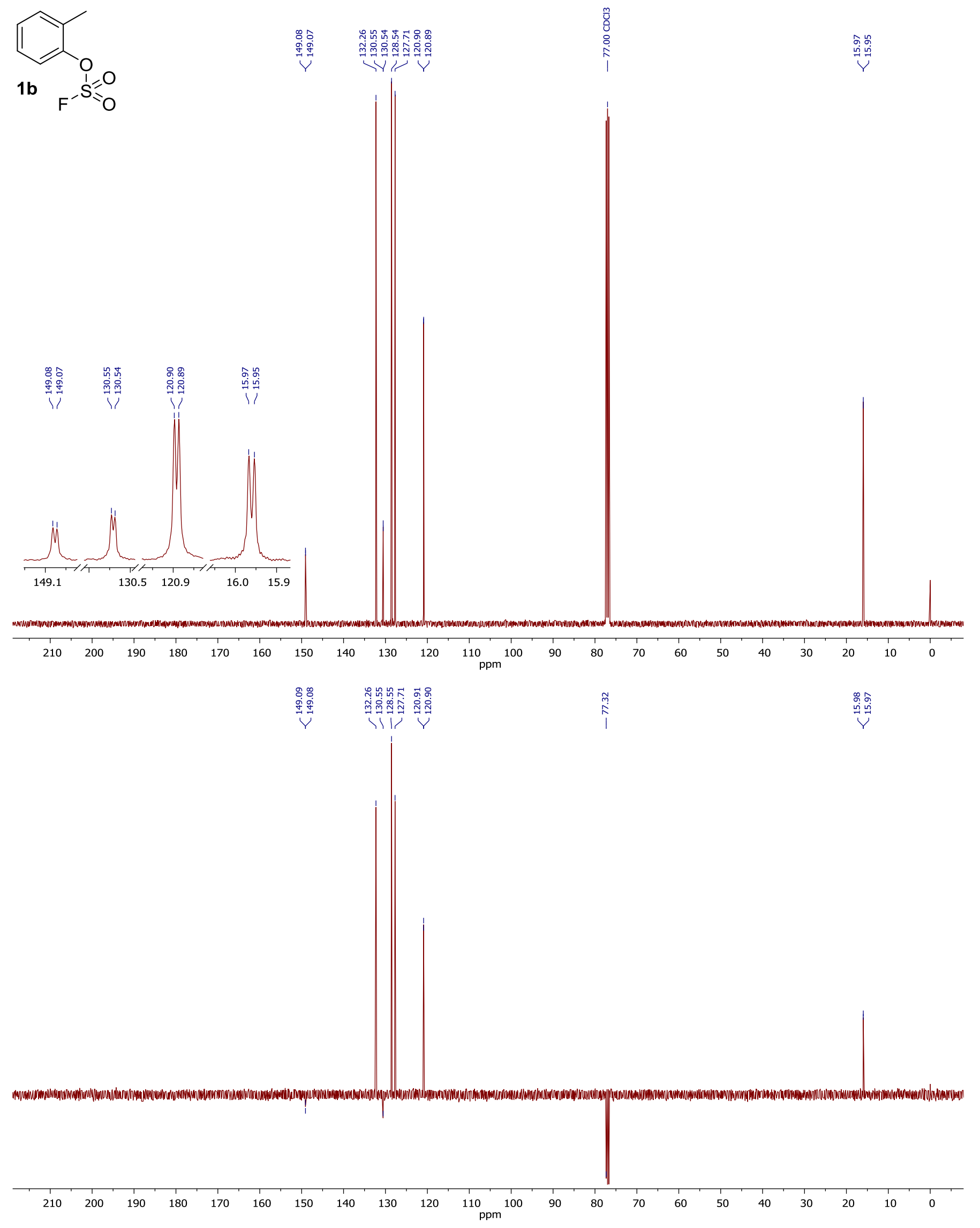


<smiles>COc1ccccc1OS(=O)(=O)F</smiles>
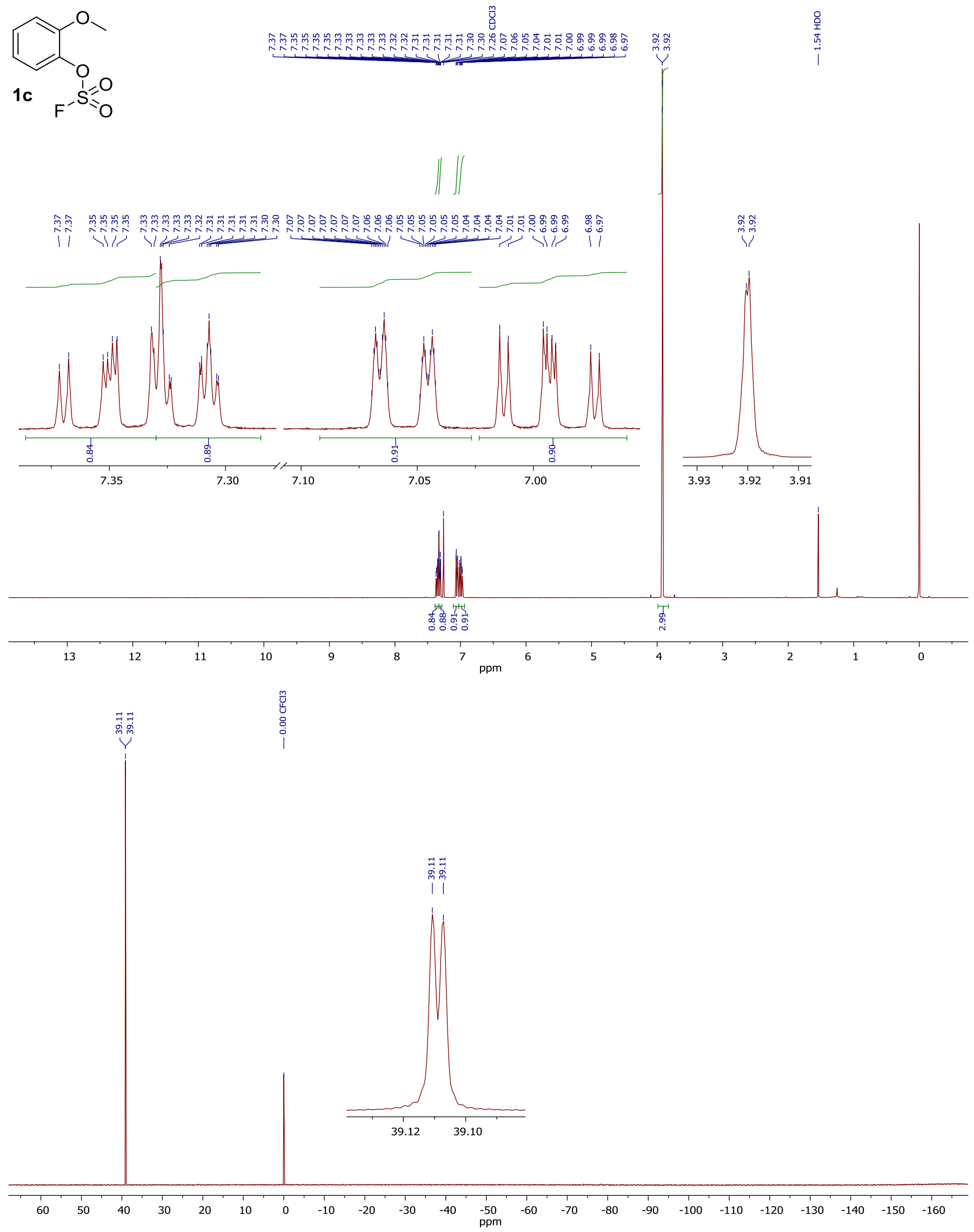


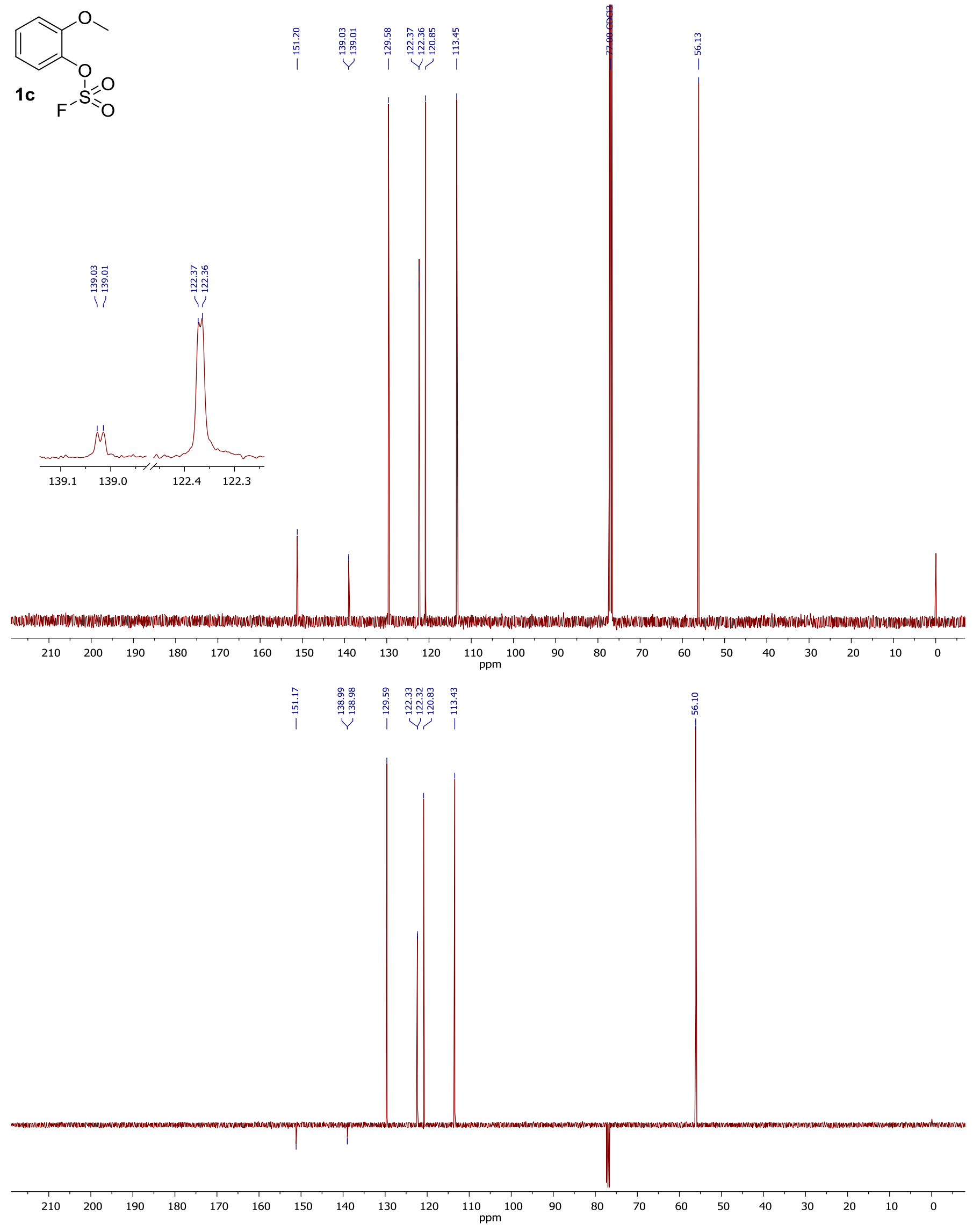



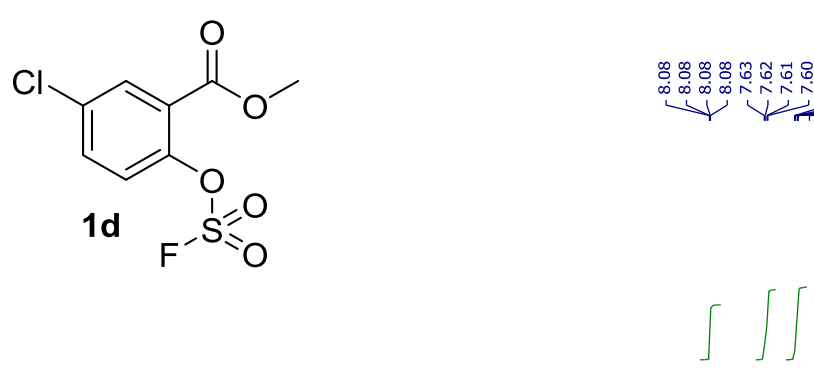

0
0
0
0
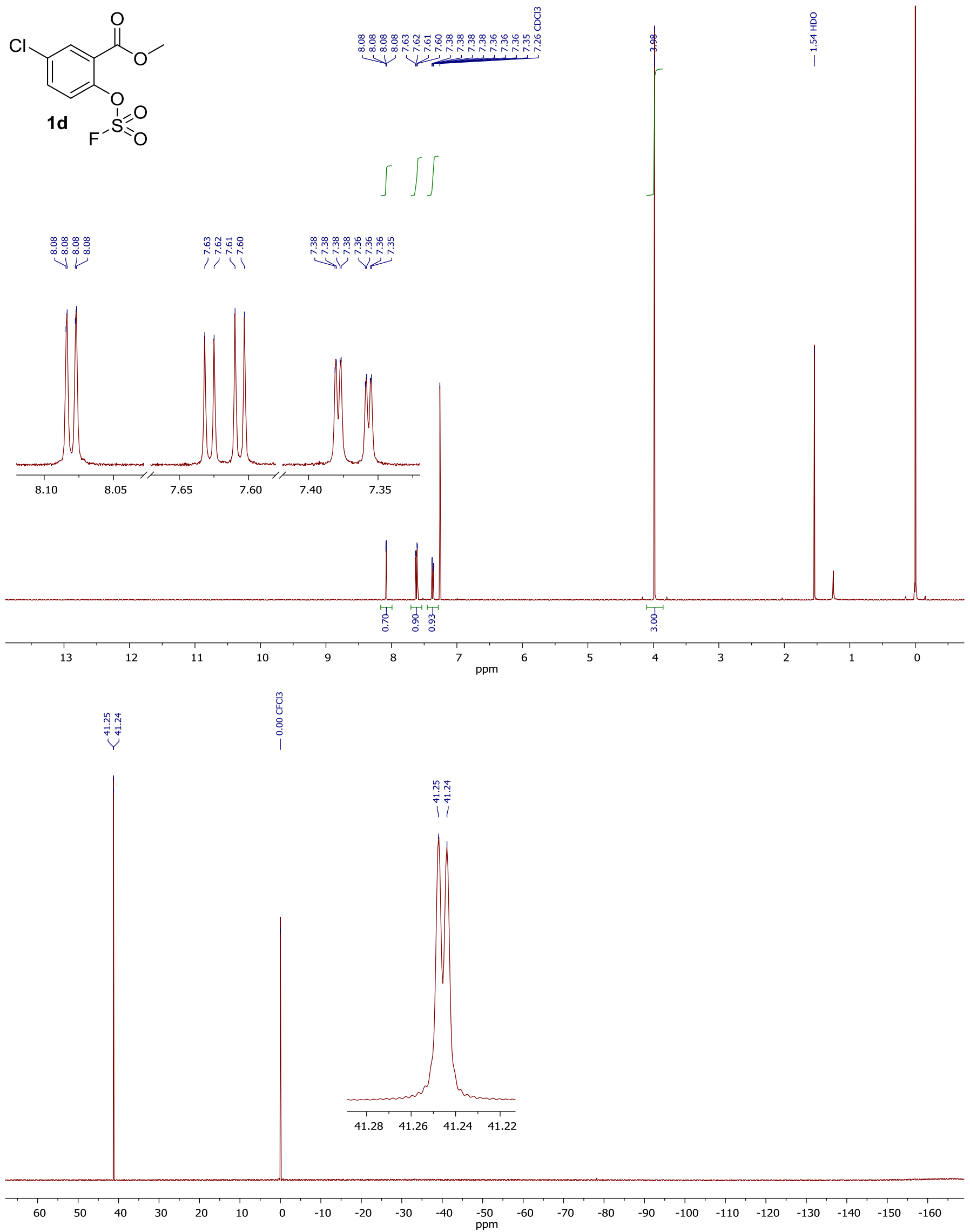

S11 

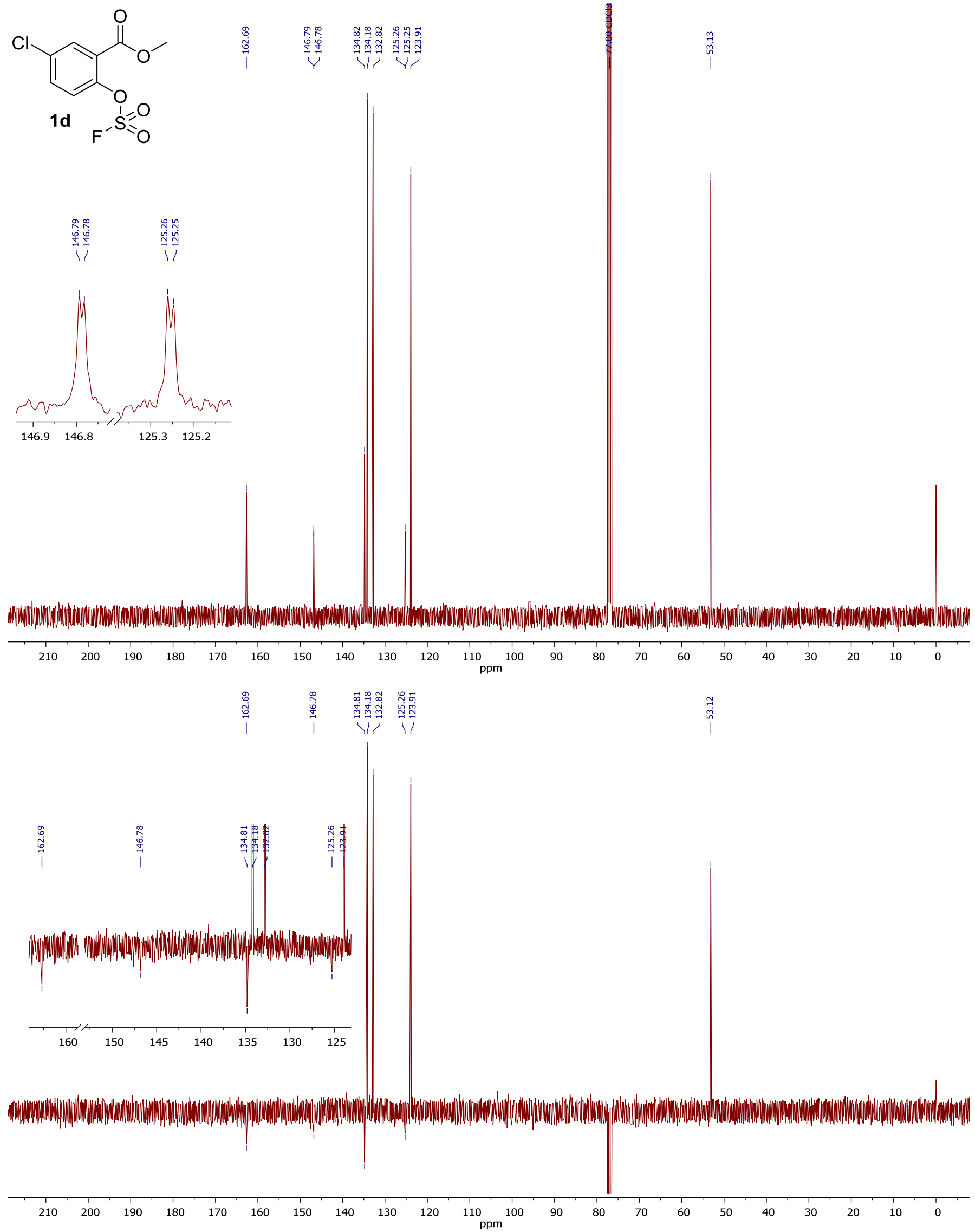

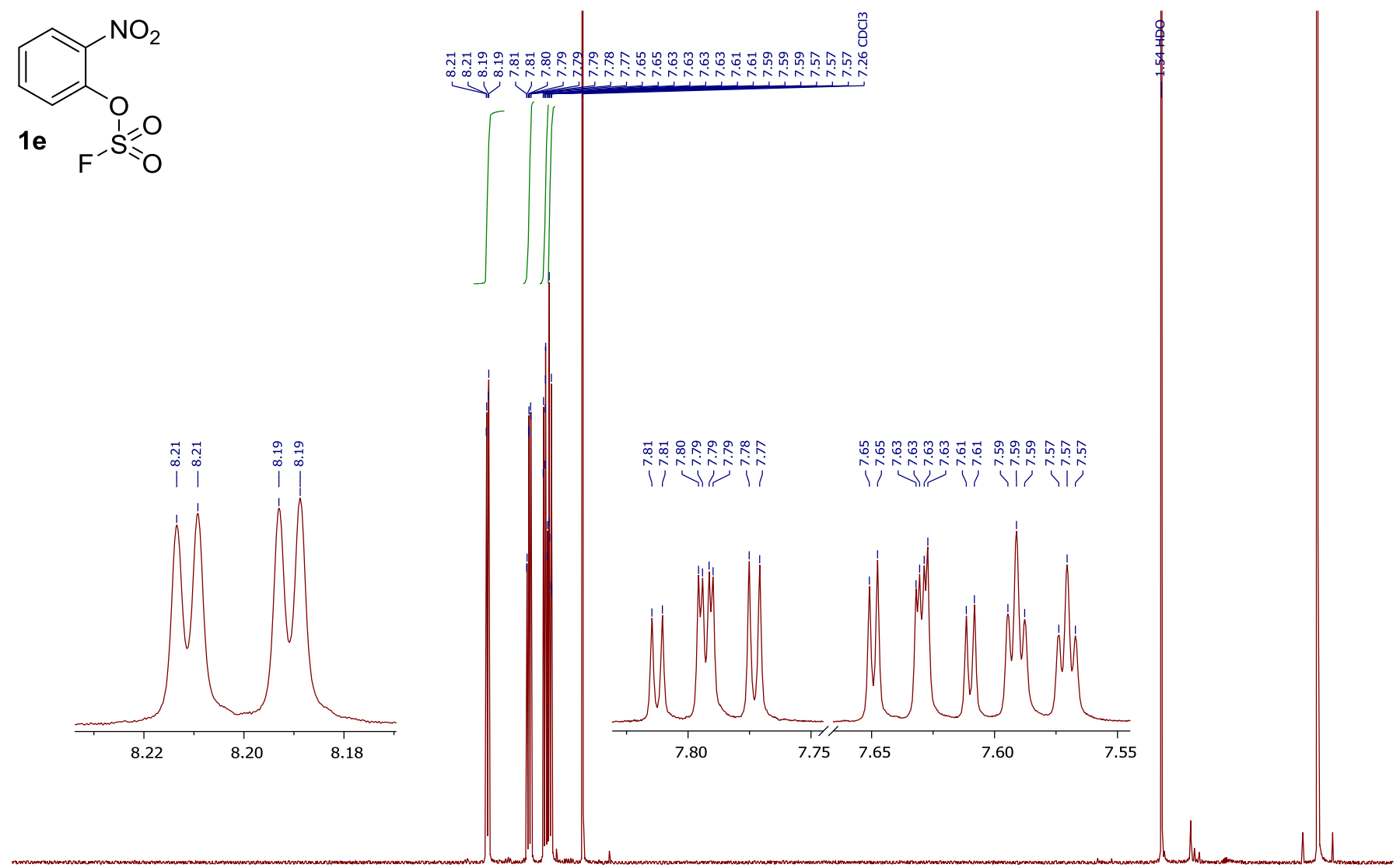

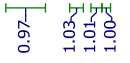

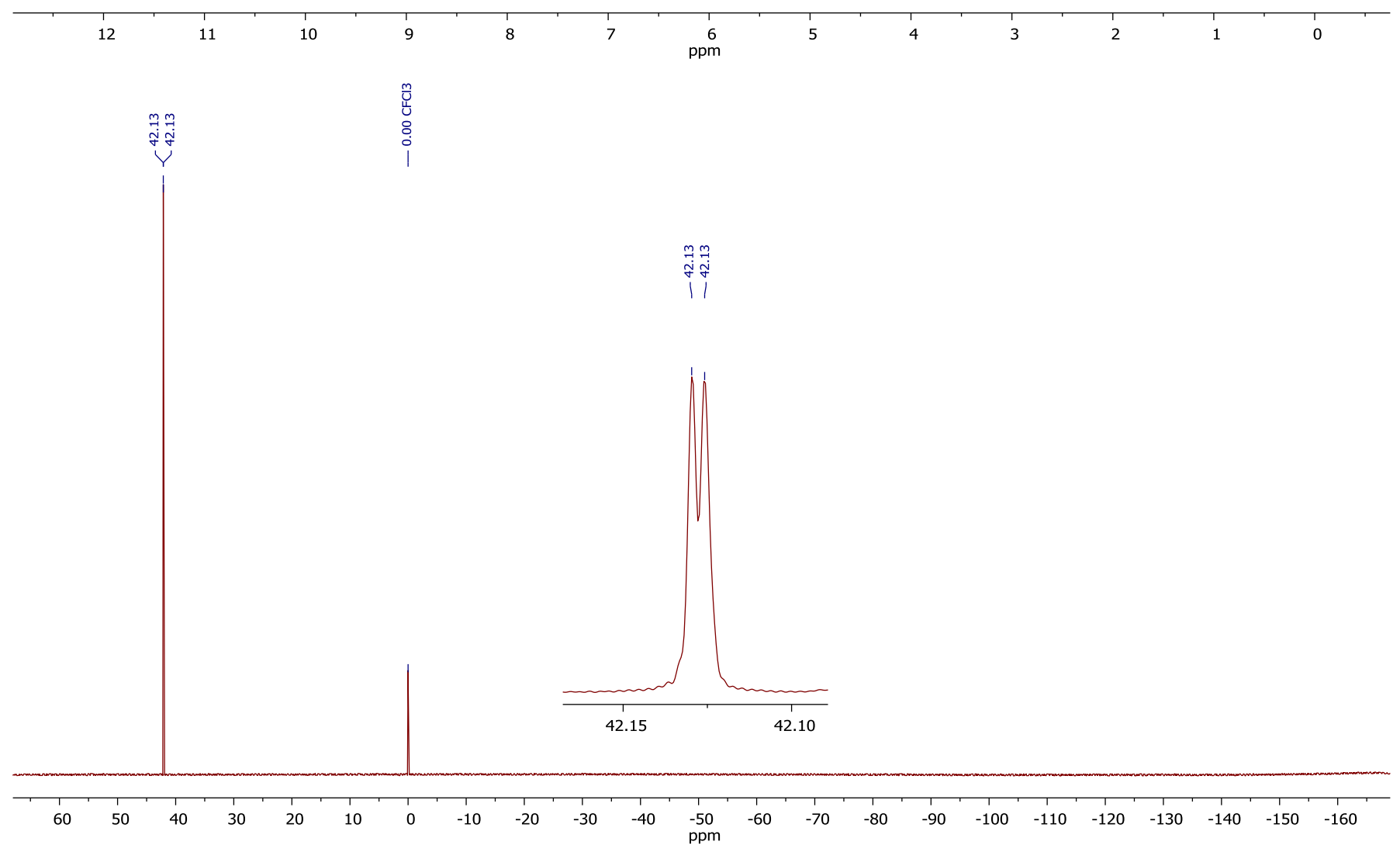




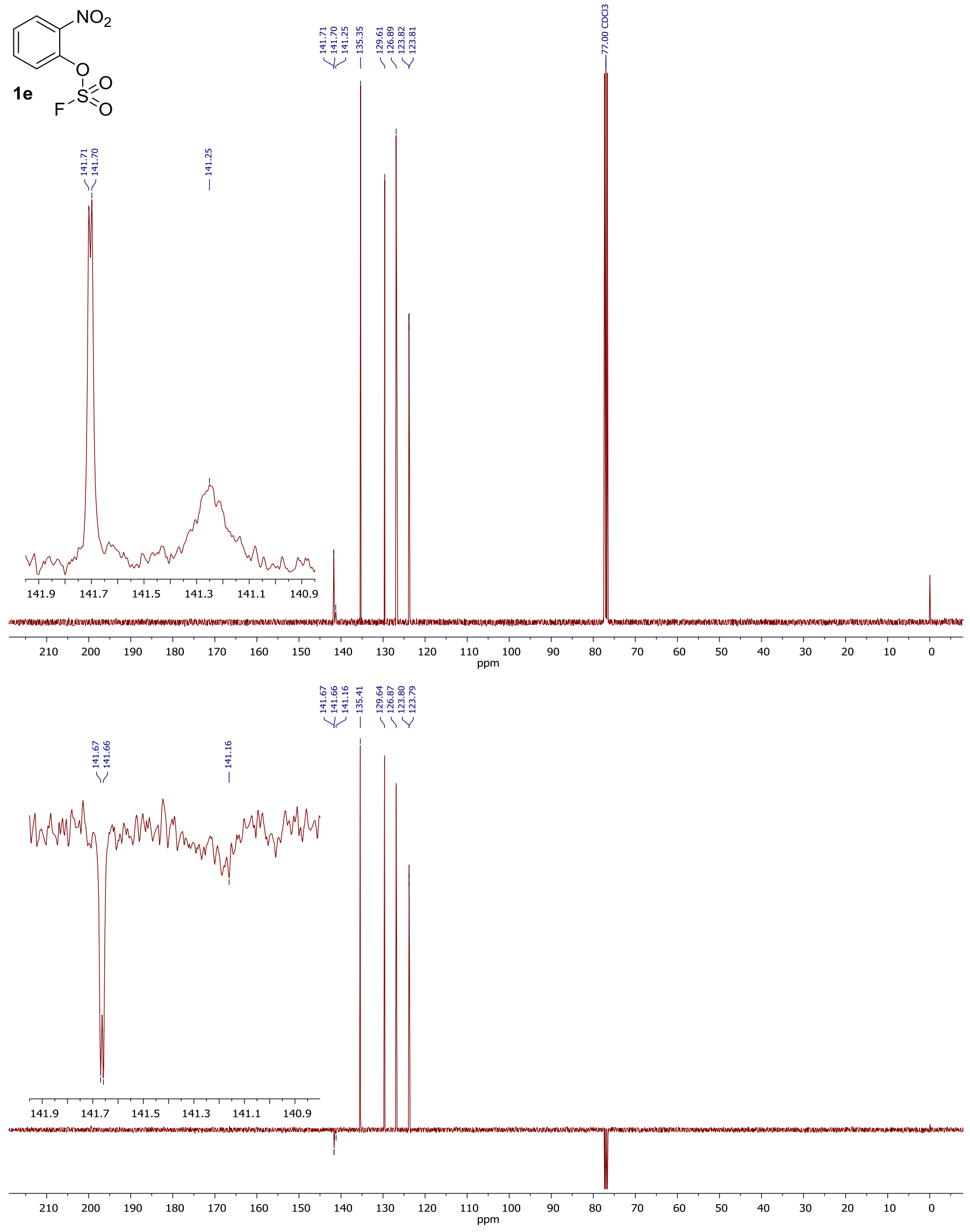


<smiles>COc1ccc(OS(=O)(=O)F)c([N+](=O)[O-])c1</smiles>
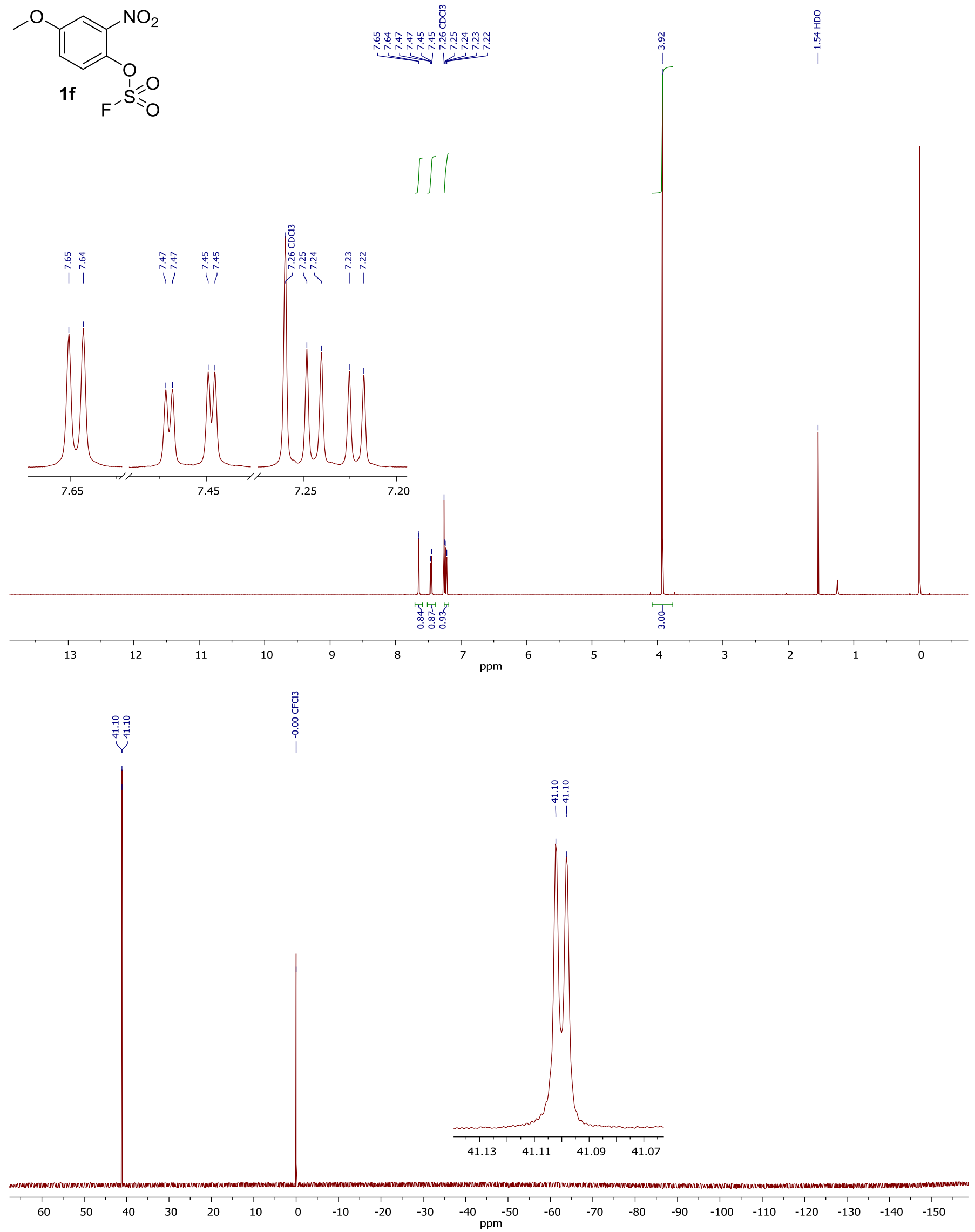

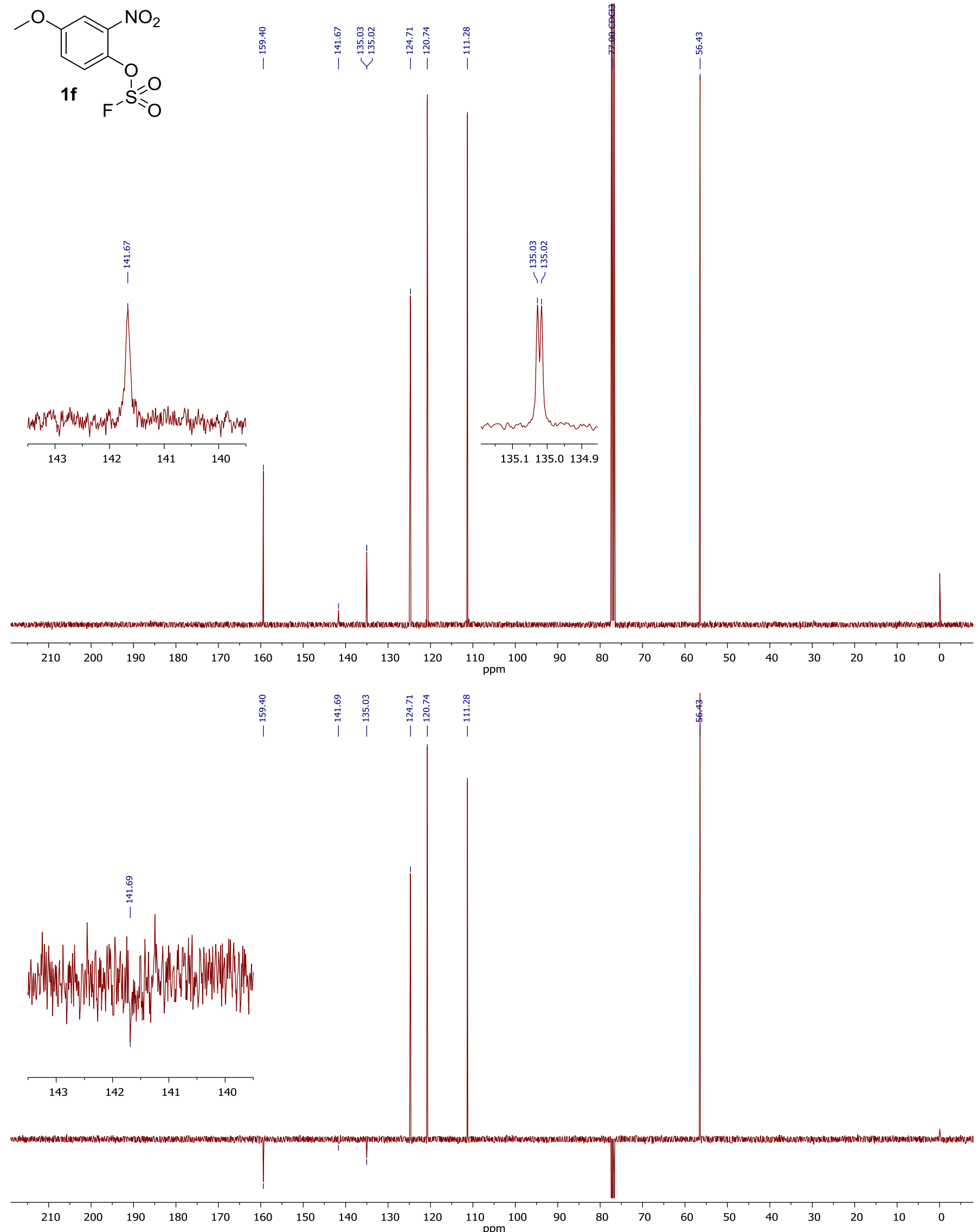


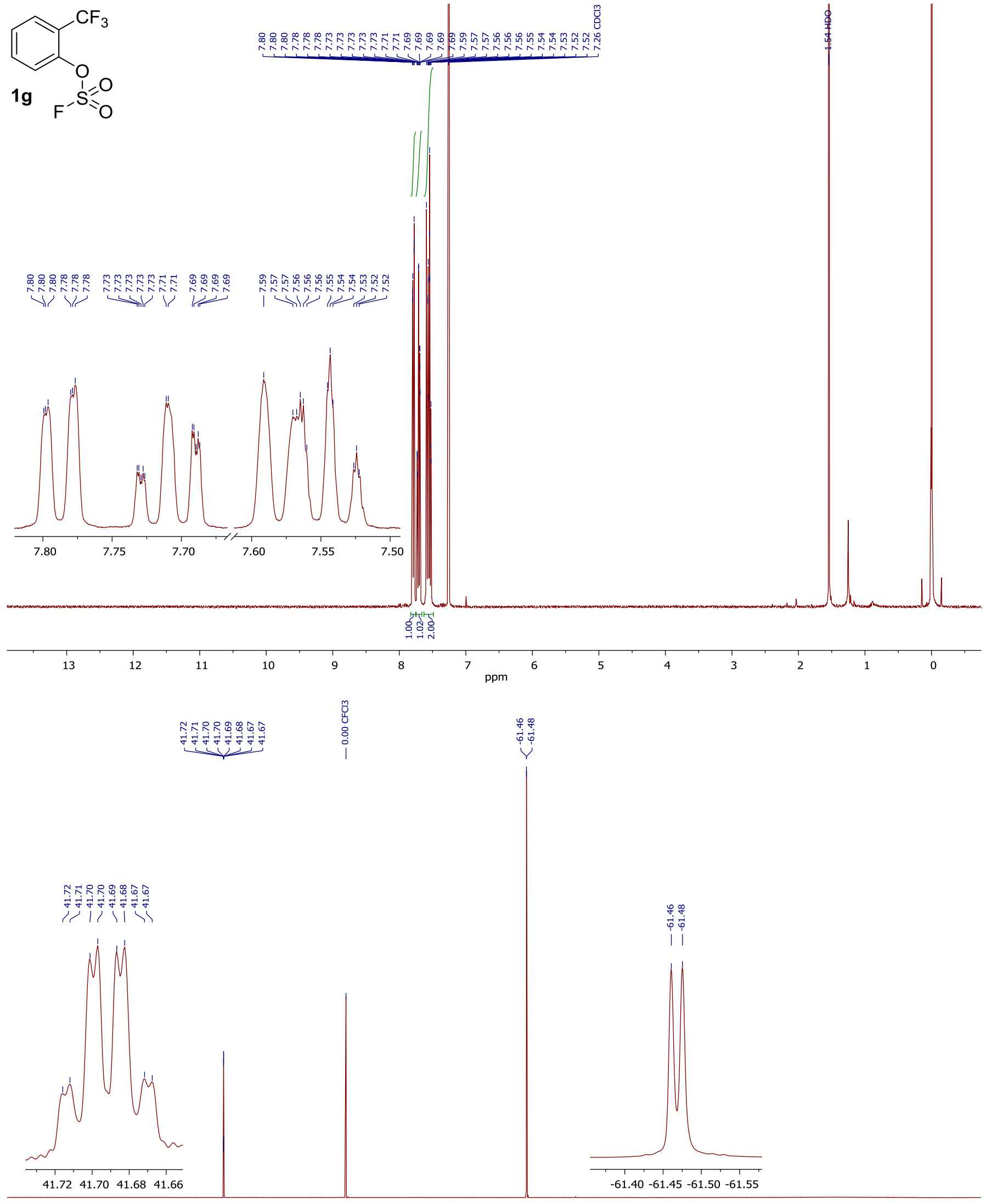

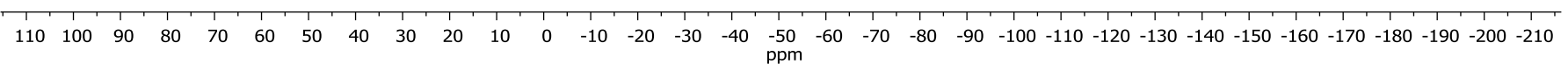


<smiles>O=S(=O)(F)Oc1ccccc1C(F)(F)F</smiles>

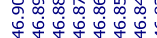

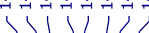

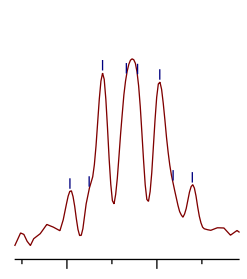

$146.90 \quad 146.85$

חై

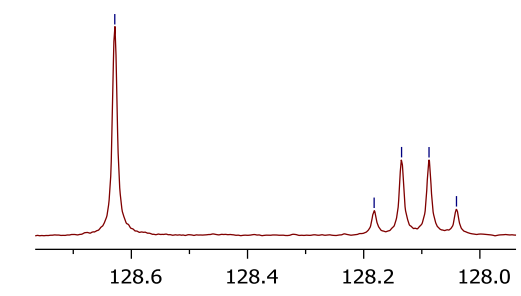

ட ஹ

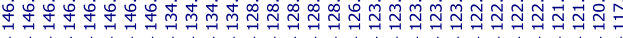
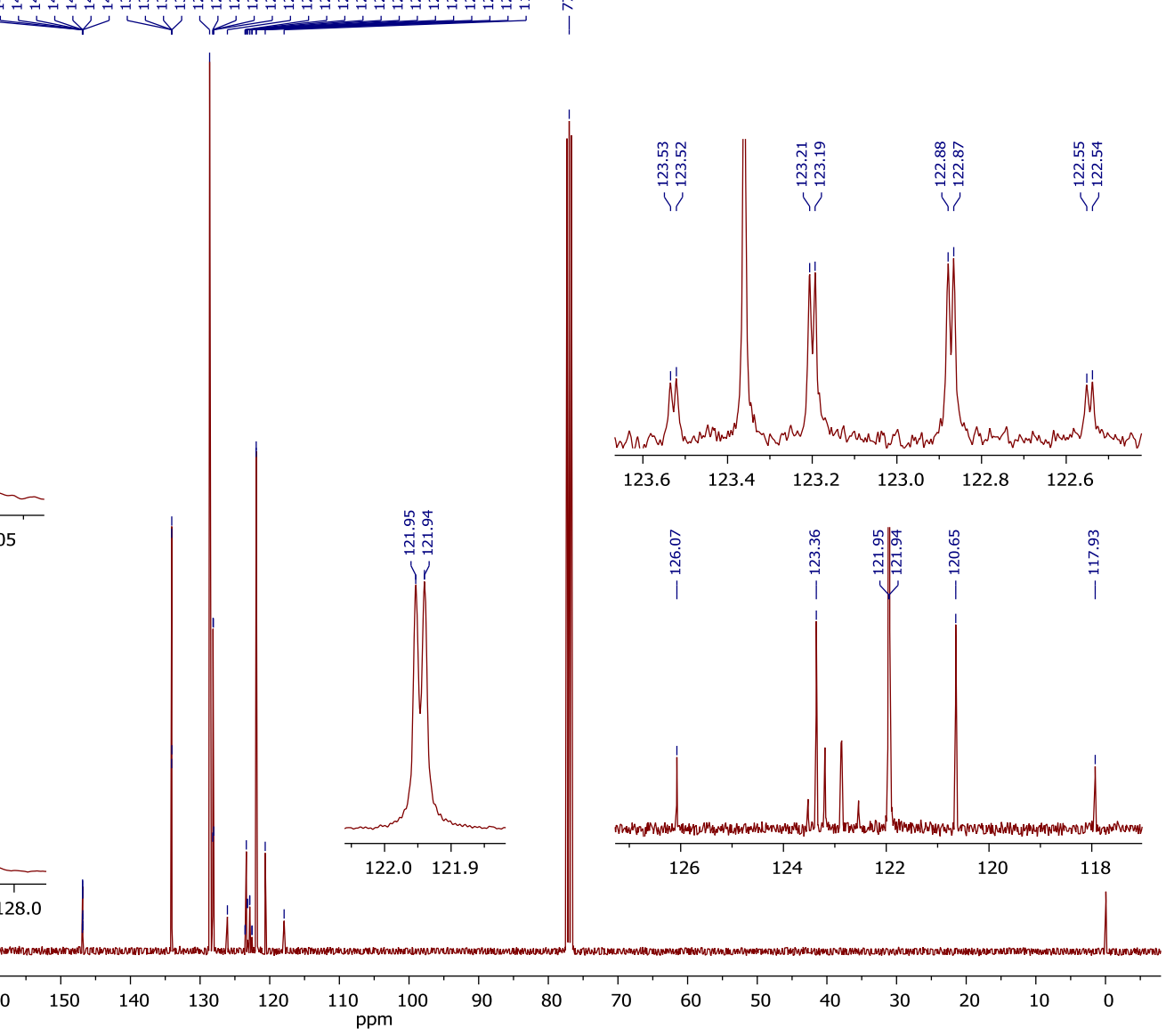

क.

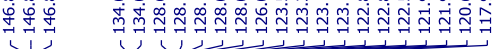

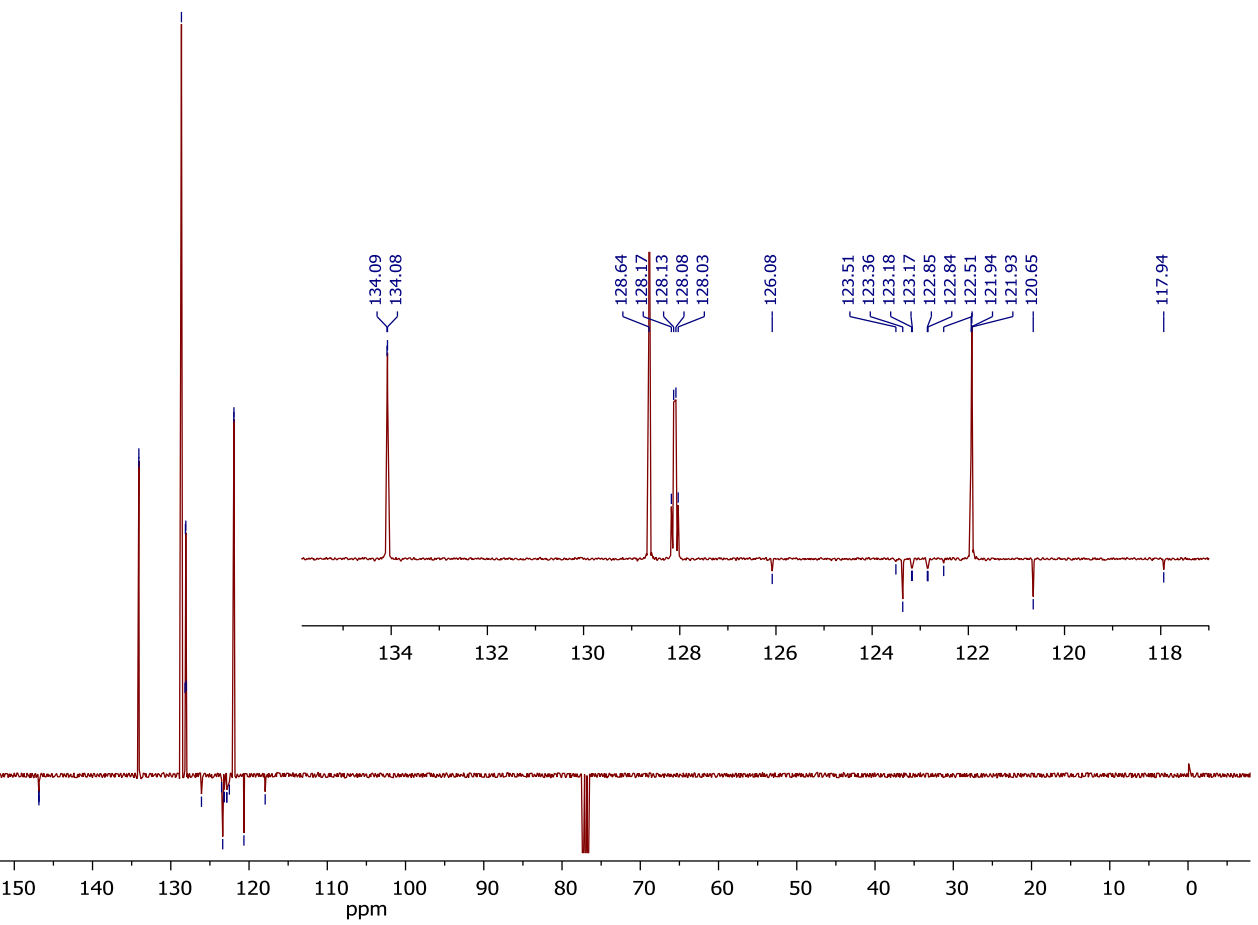


<smiles>O=S(=O)(F)Oc1cc(F)ccc1C(F)(F)F</smiles>

吕

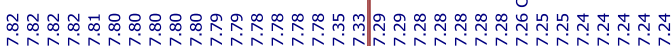
จุ

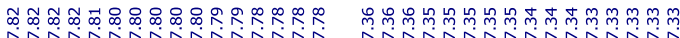

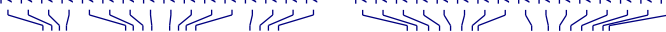
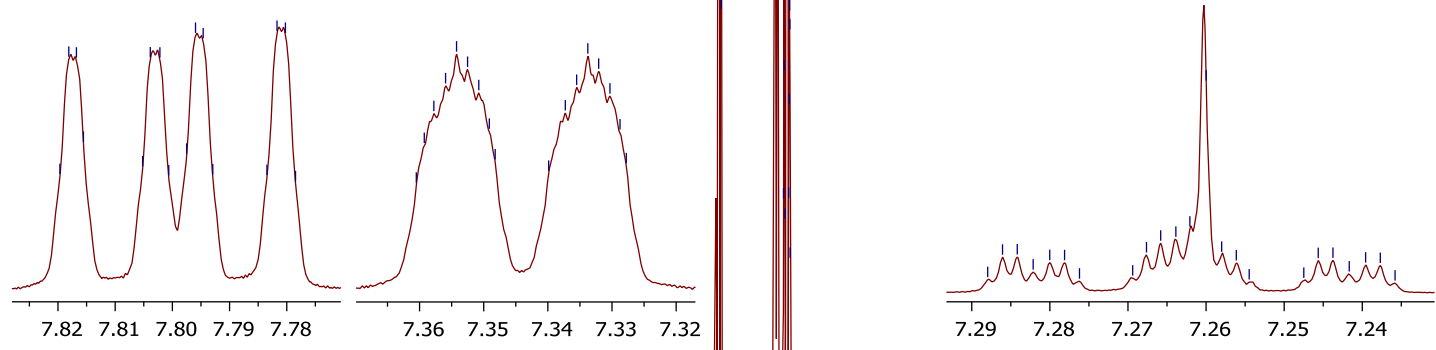

1. Ill hu 1 1 गु

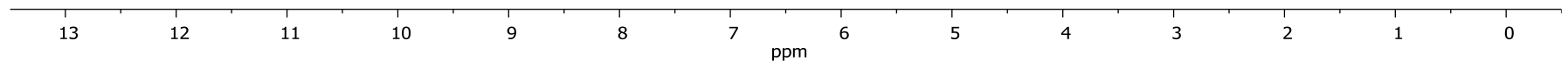
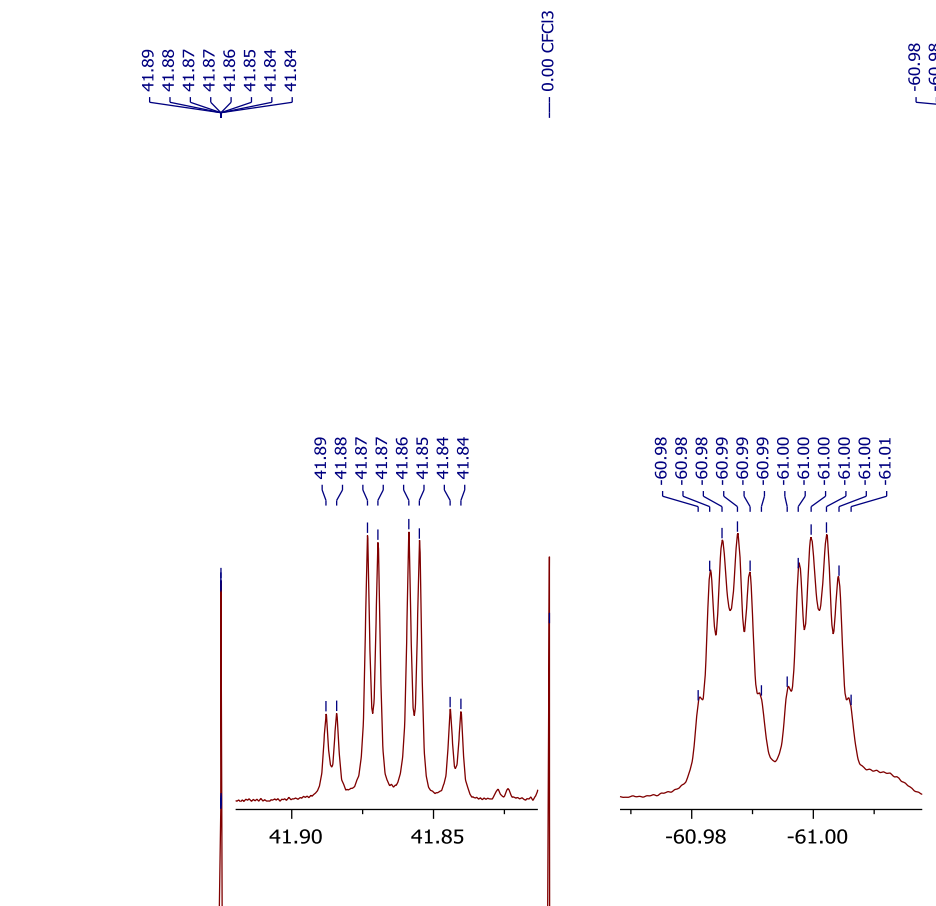

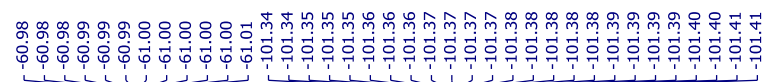
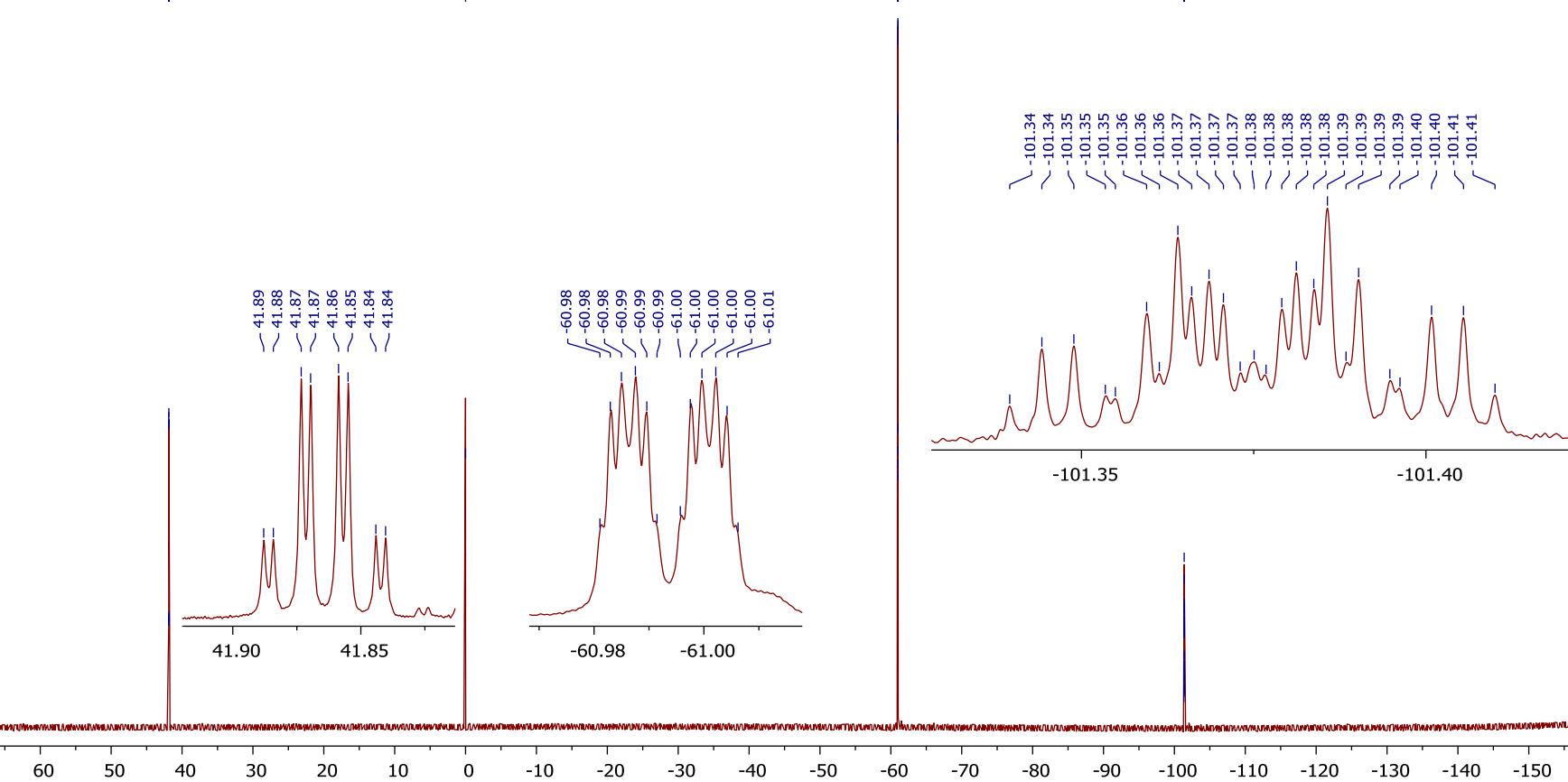

$-40 p^{-50}$ 


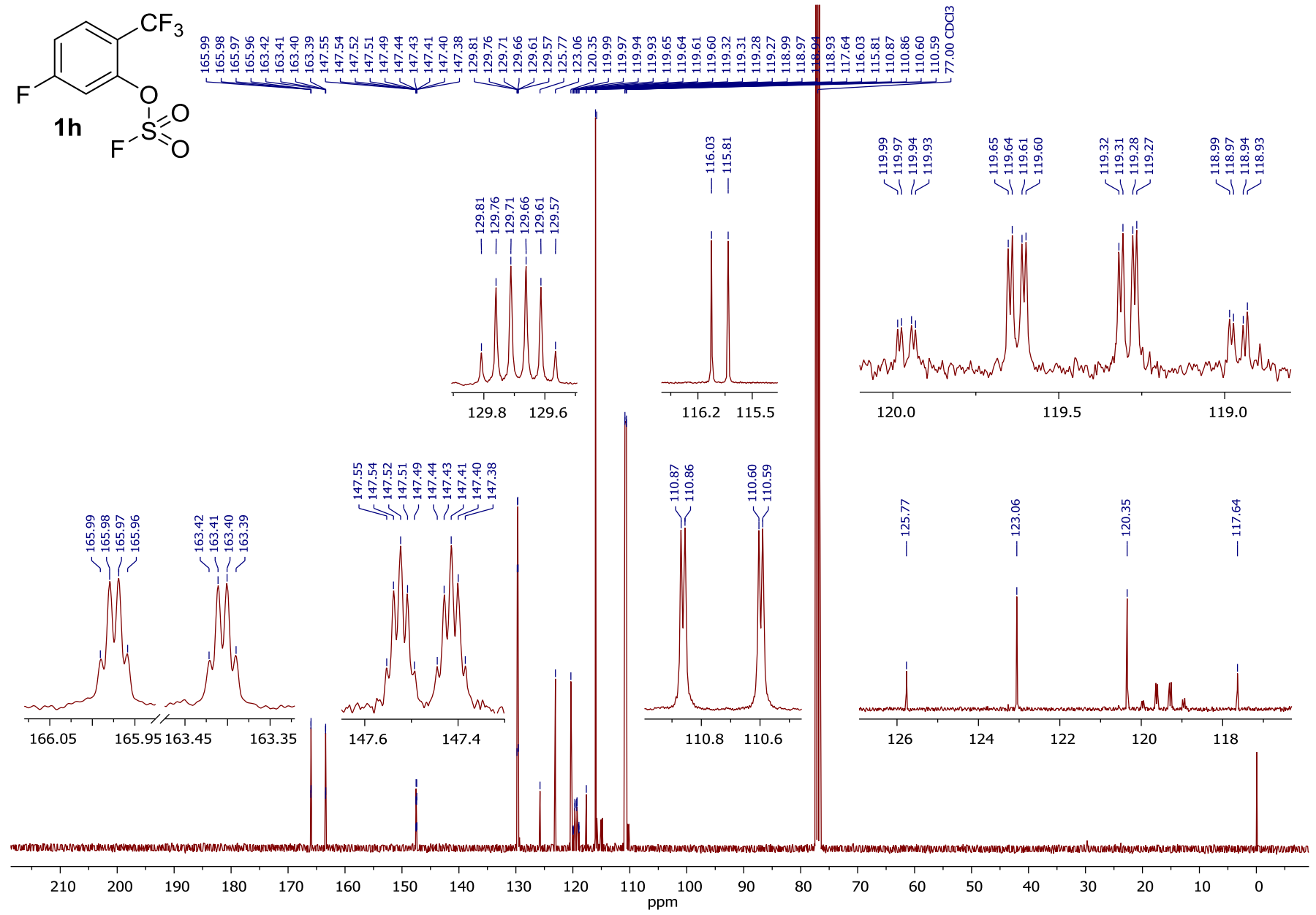

V|

V)

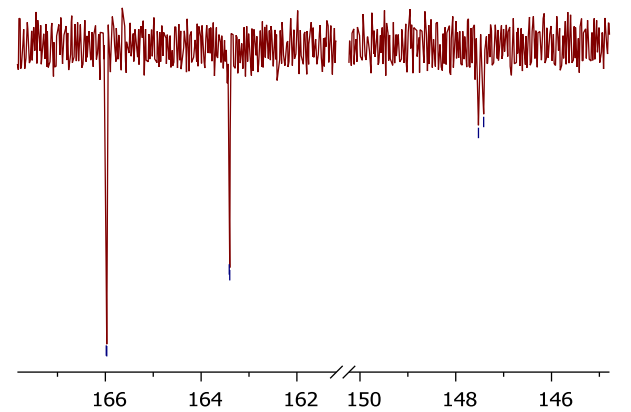

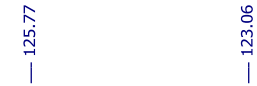

|

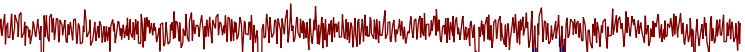

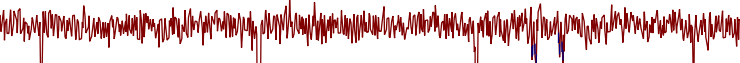

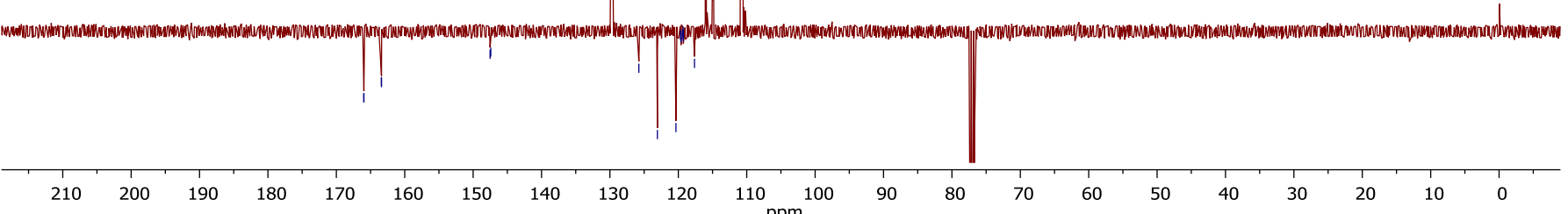



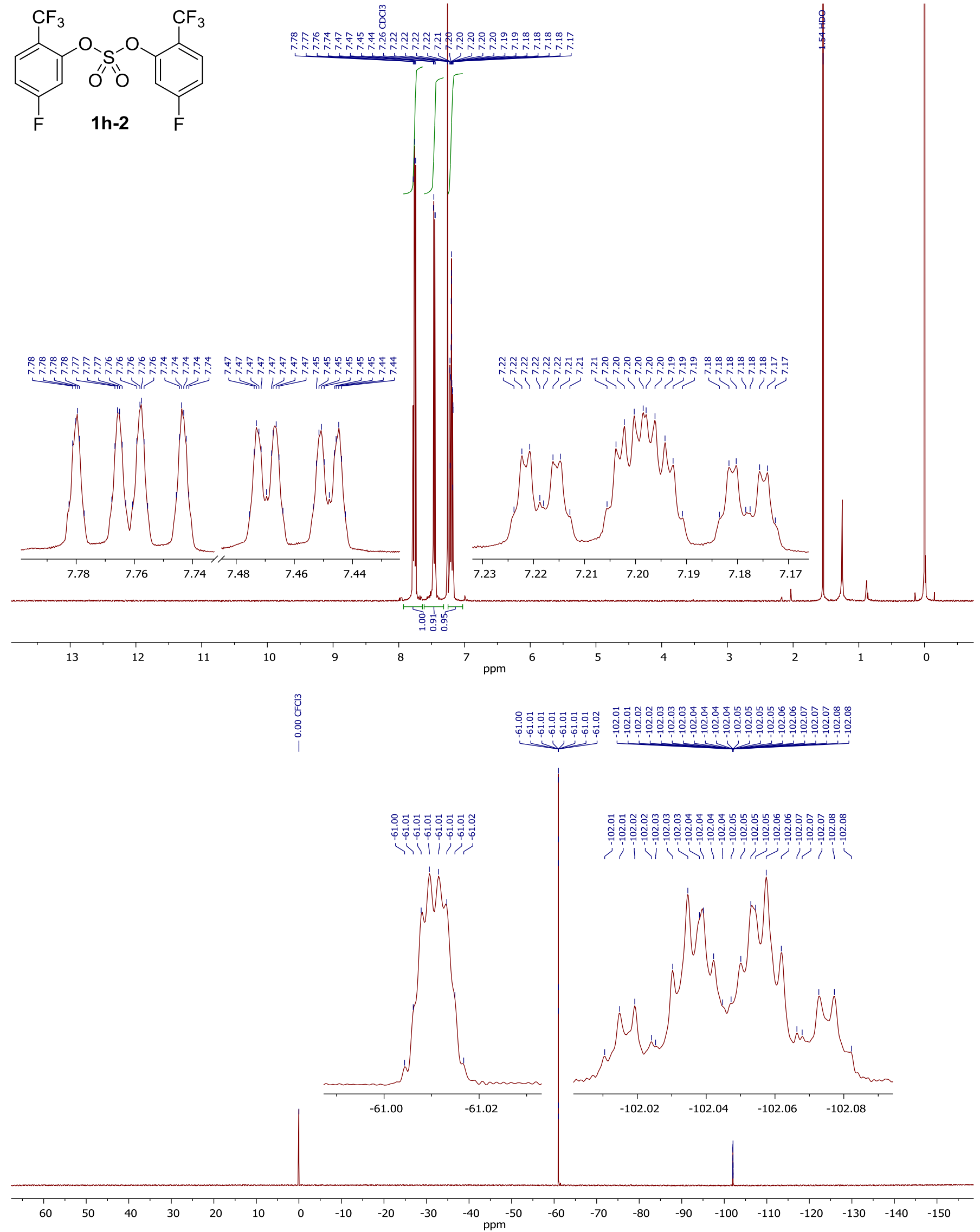

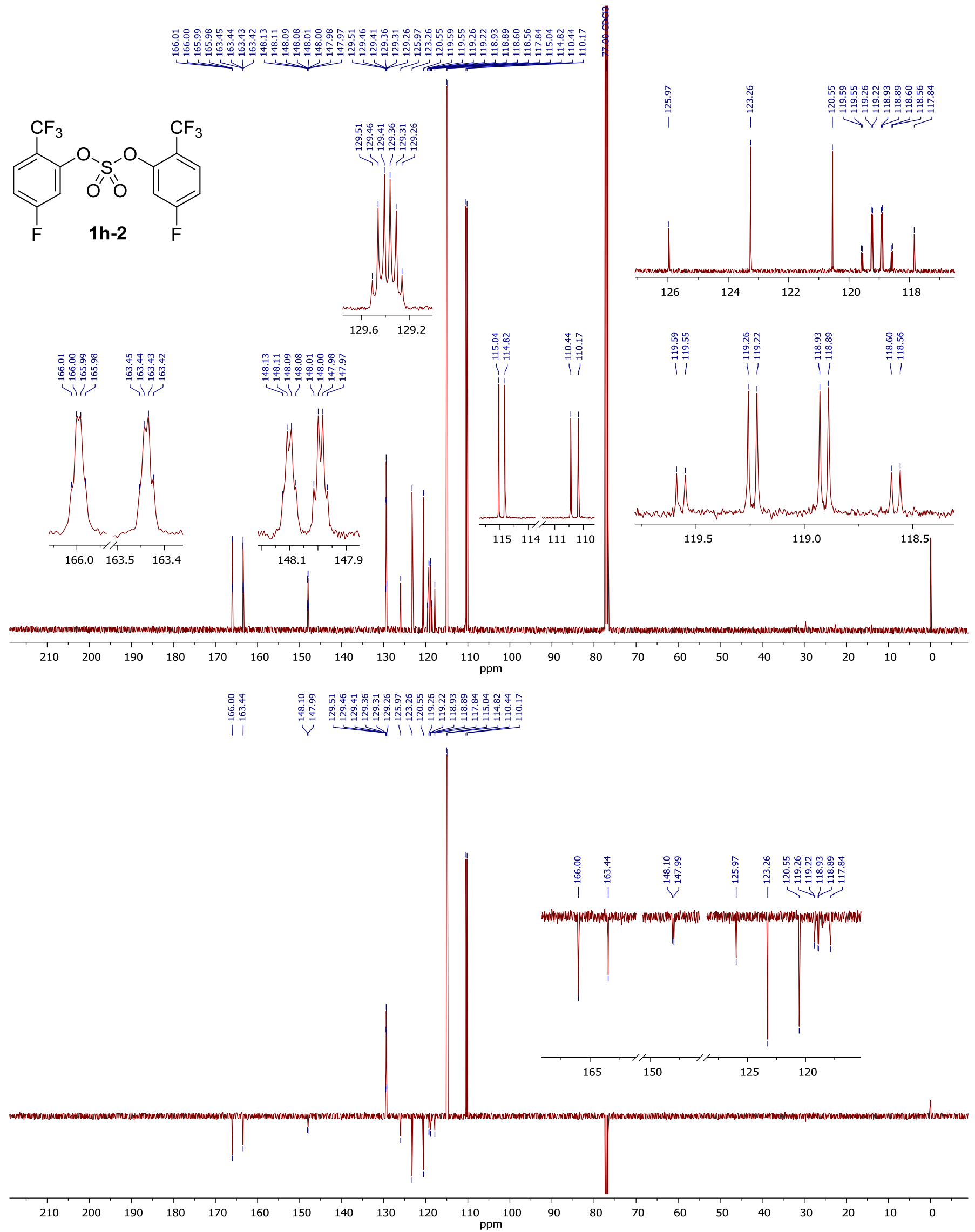


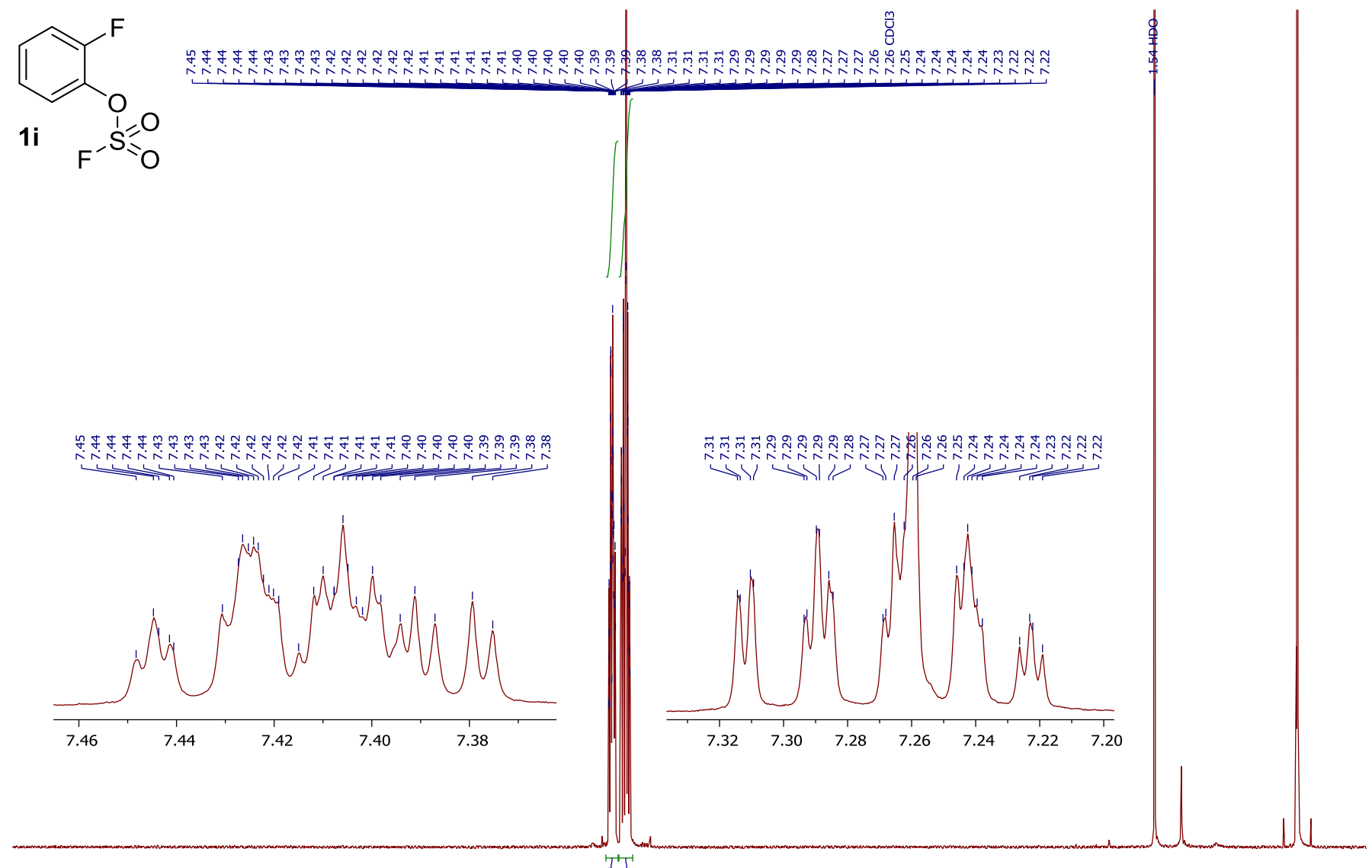

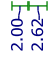
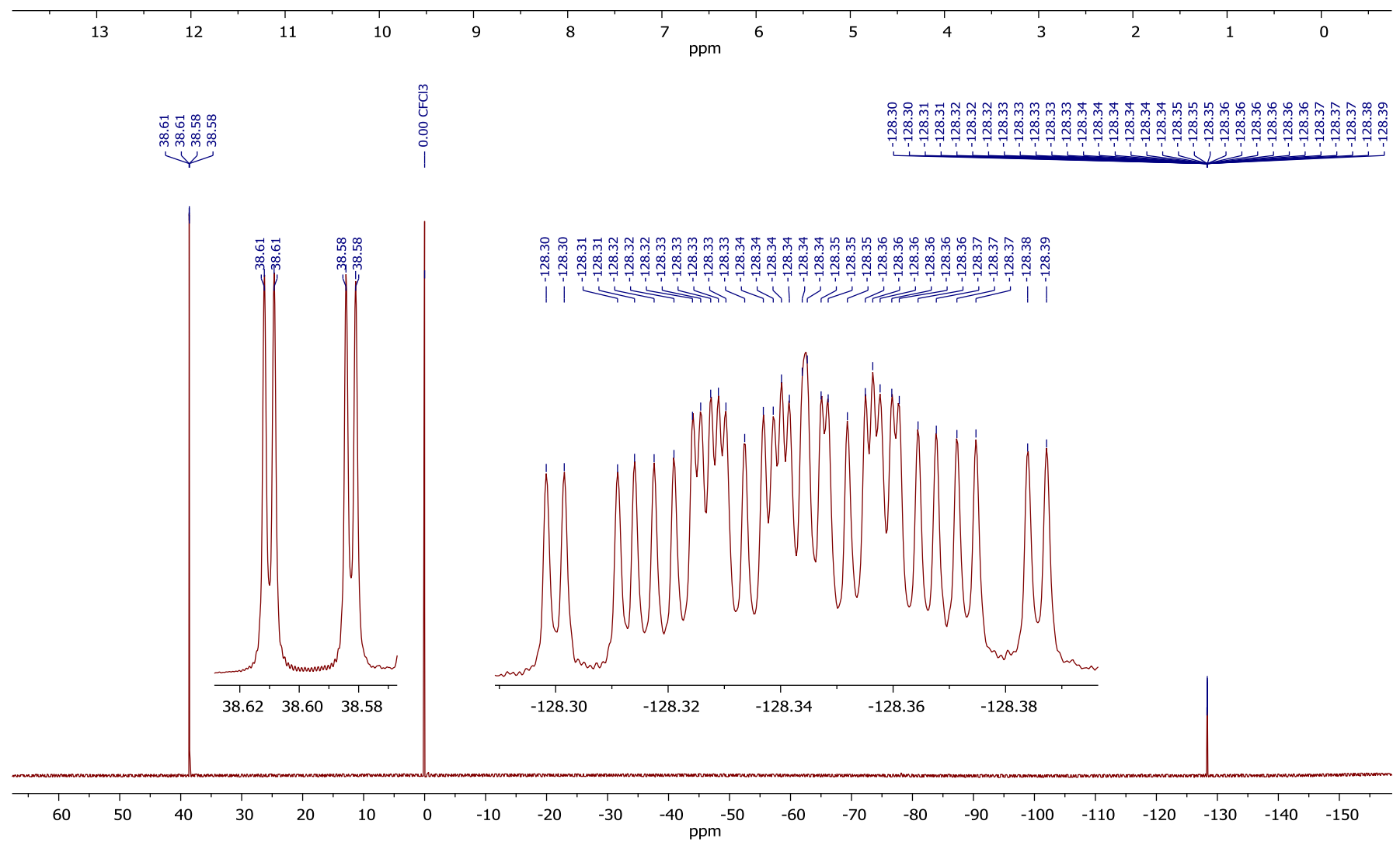
<smiles>O=S(=O)(F)Oc1ccccc1F</smiles>

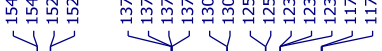

1i $\quad F^{-} \stackrel{1}{=}=0$
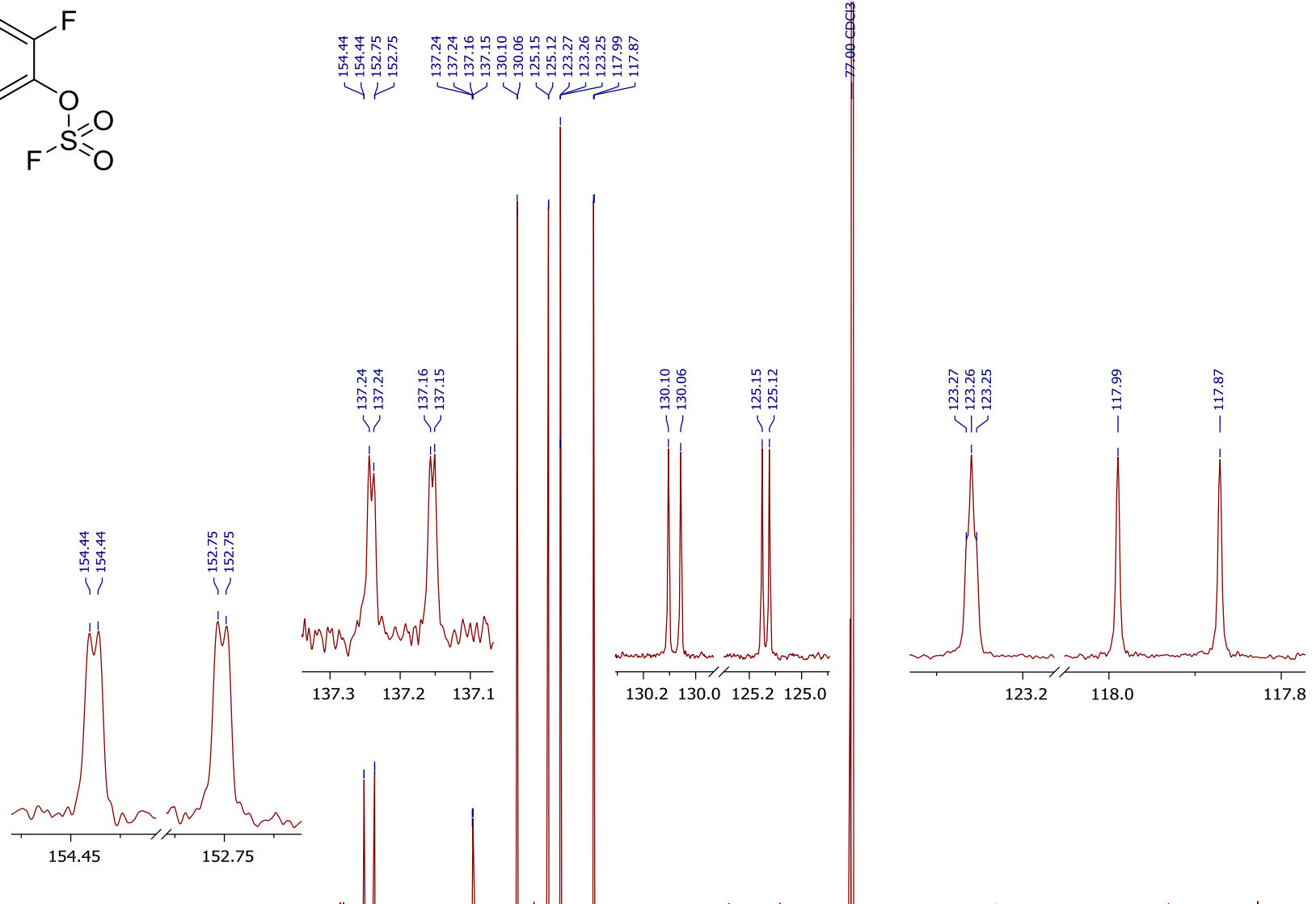

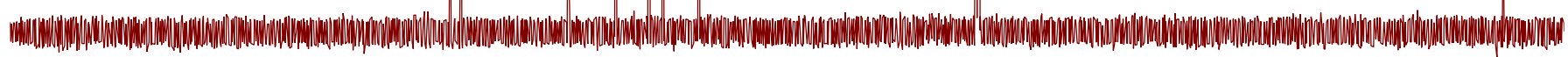

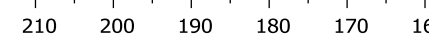

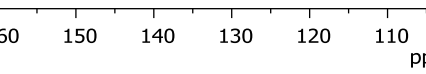

$\mathrm{ppm}^{100}$

80

60

$50 \quad 40$

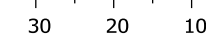

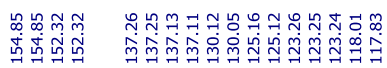

$\checkmark V$

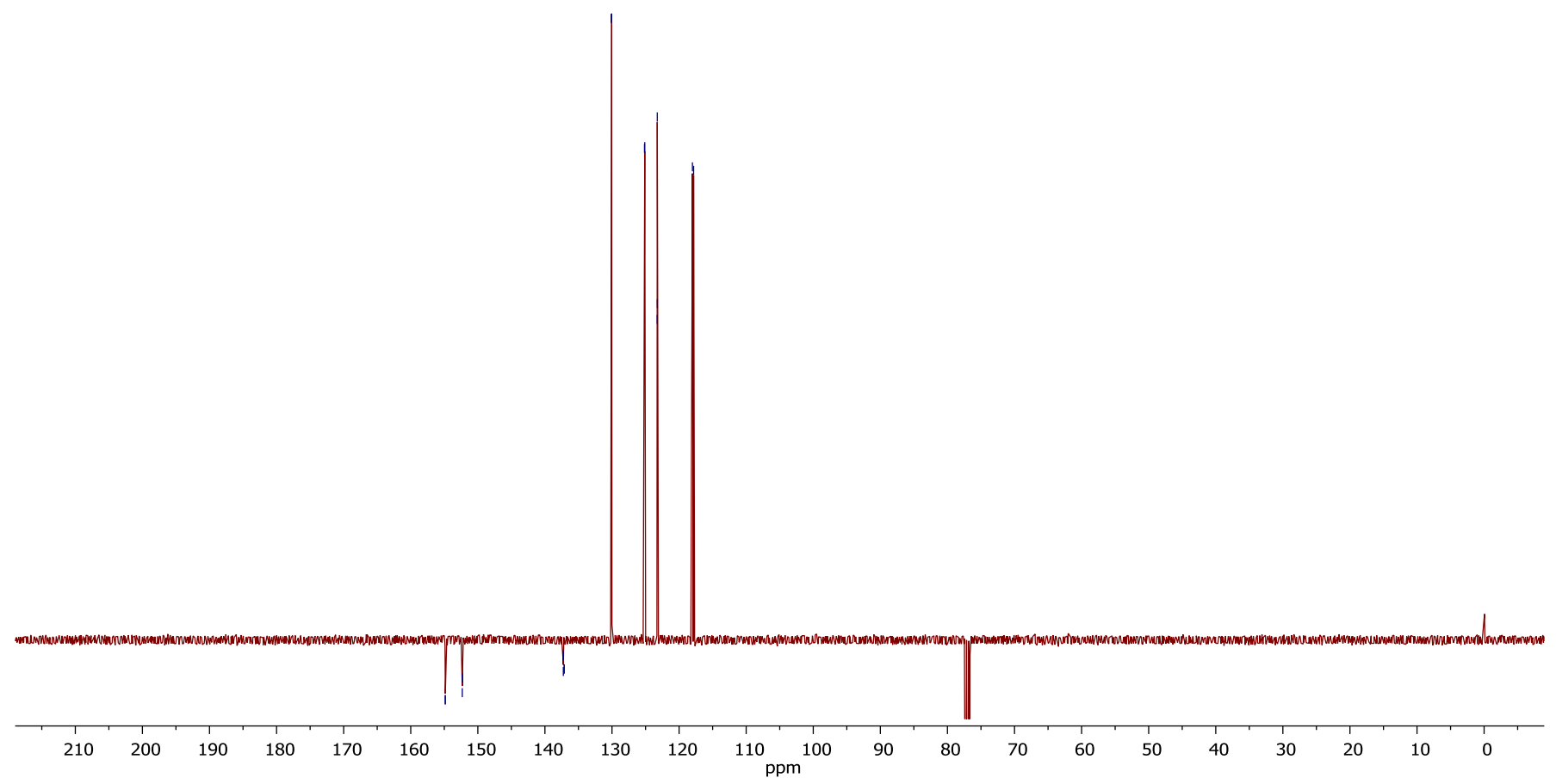



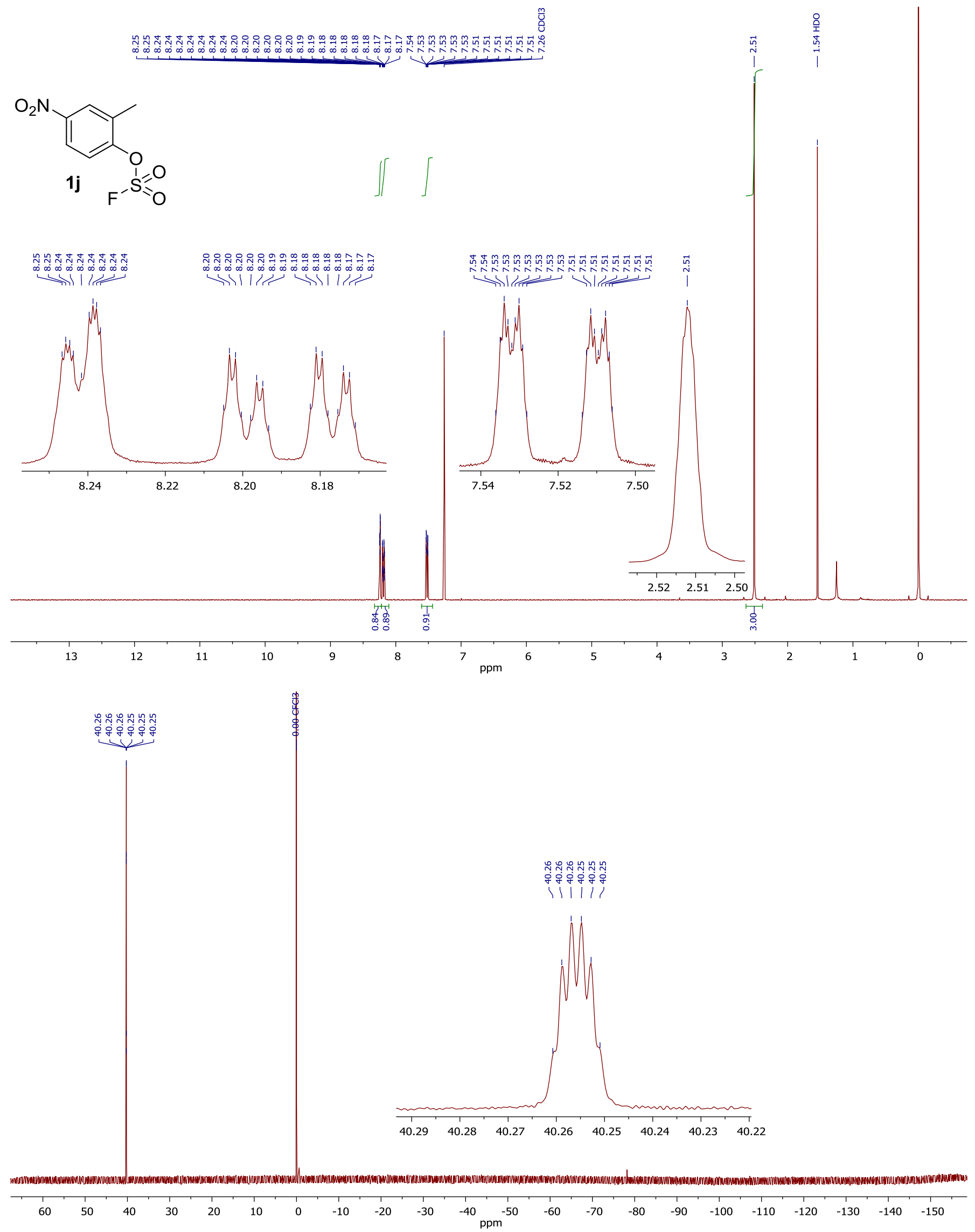

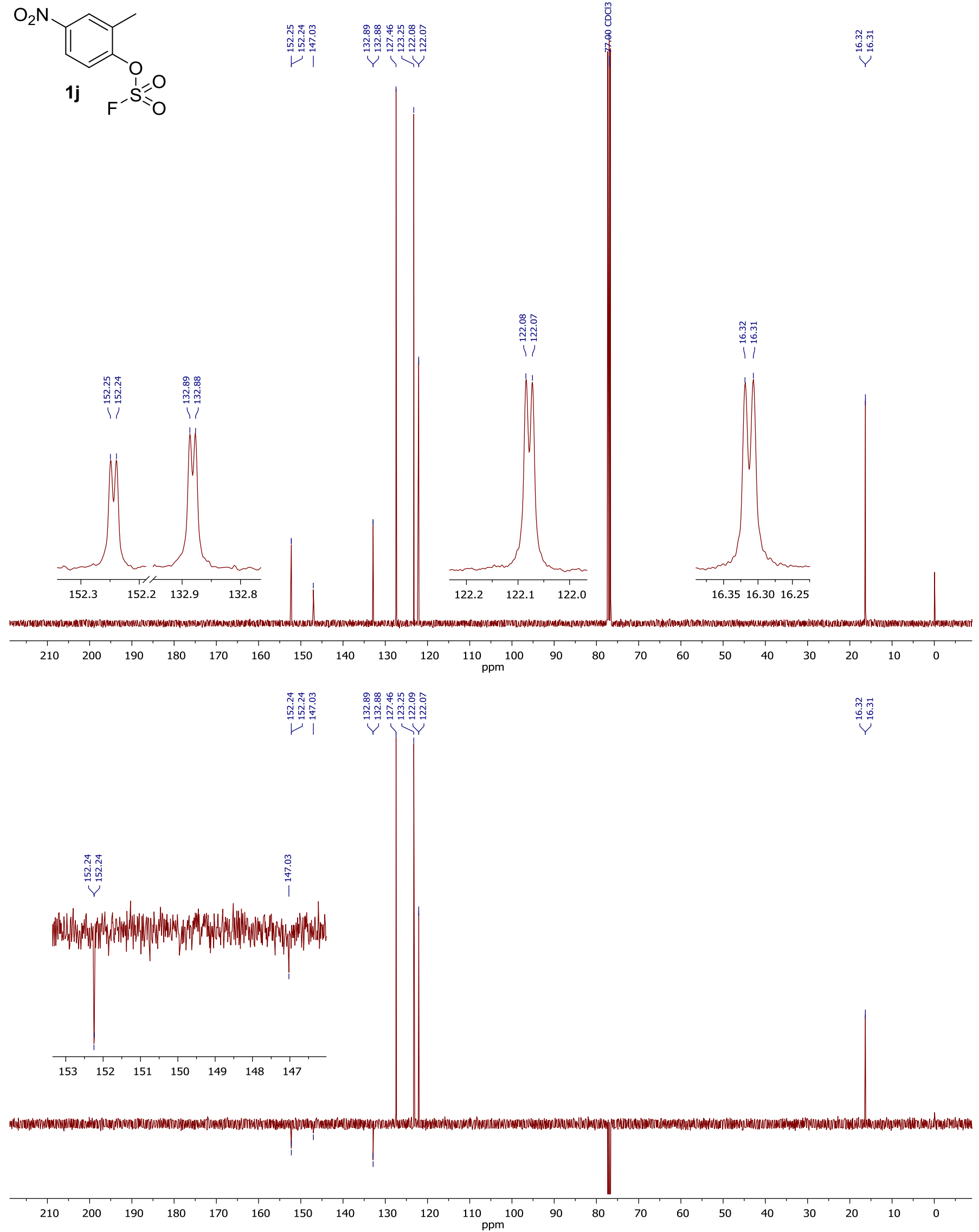
<smiles>CC(=O)c1ccc(OS(=O)(=O)F)c(C)c1</smiles>
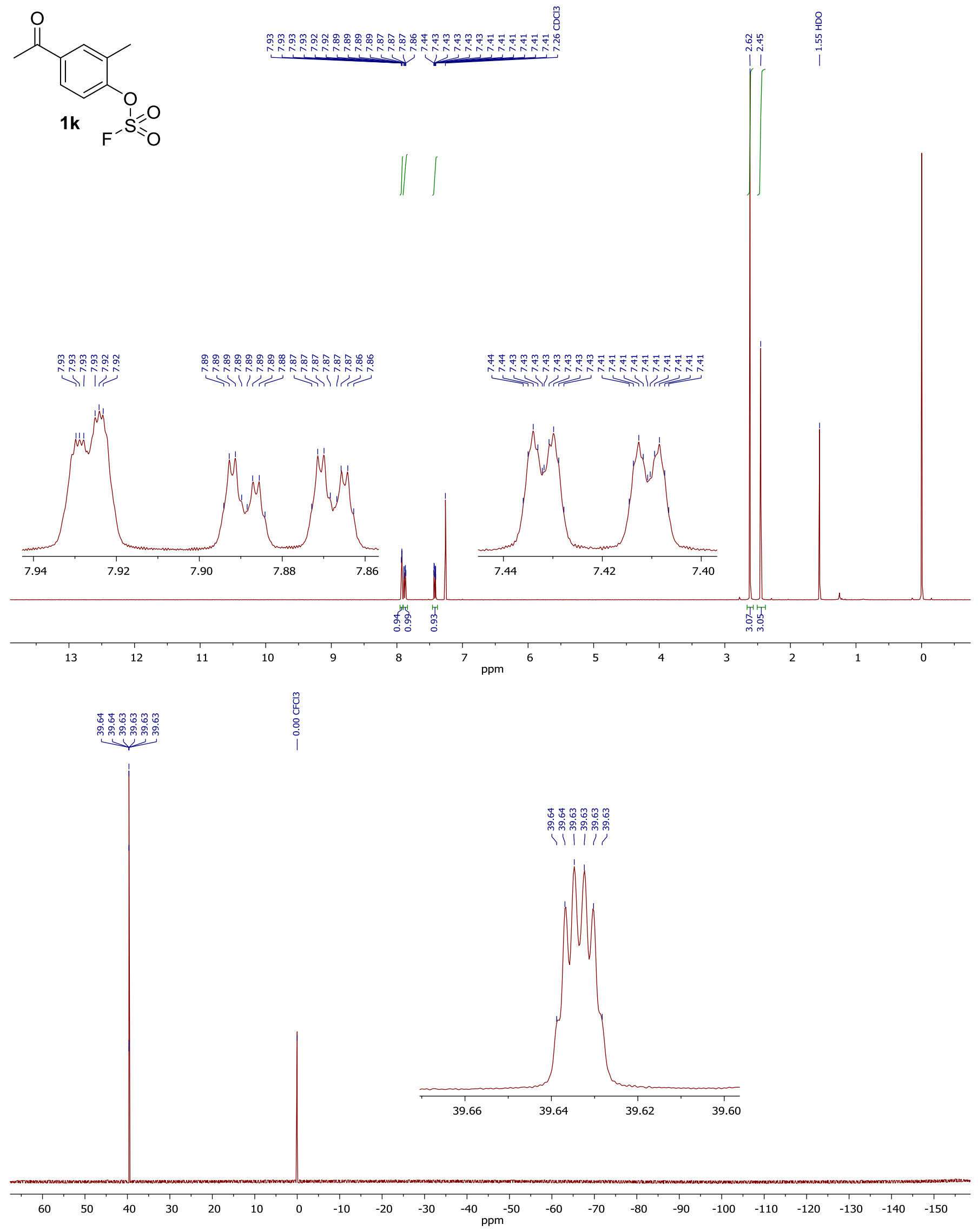

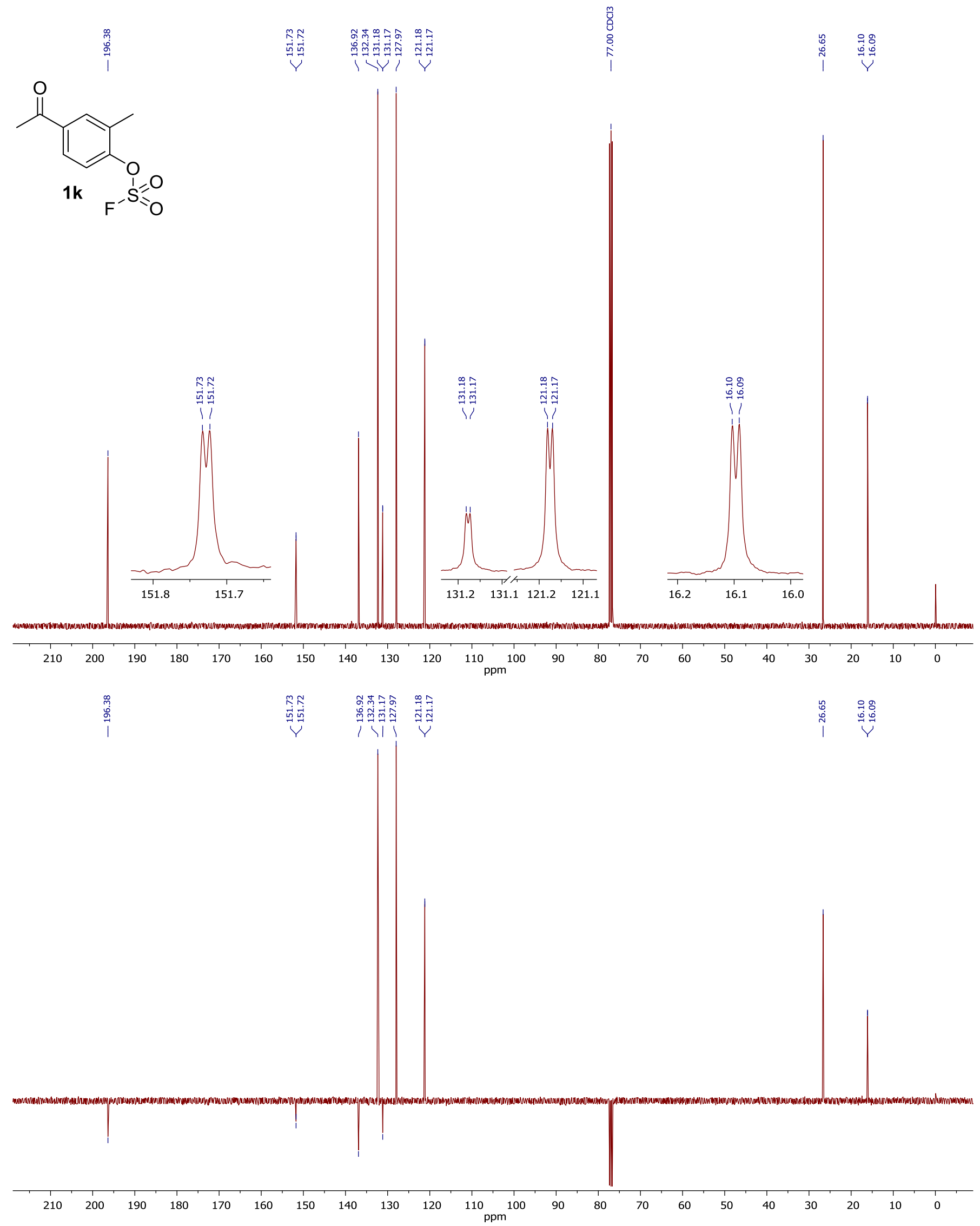

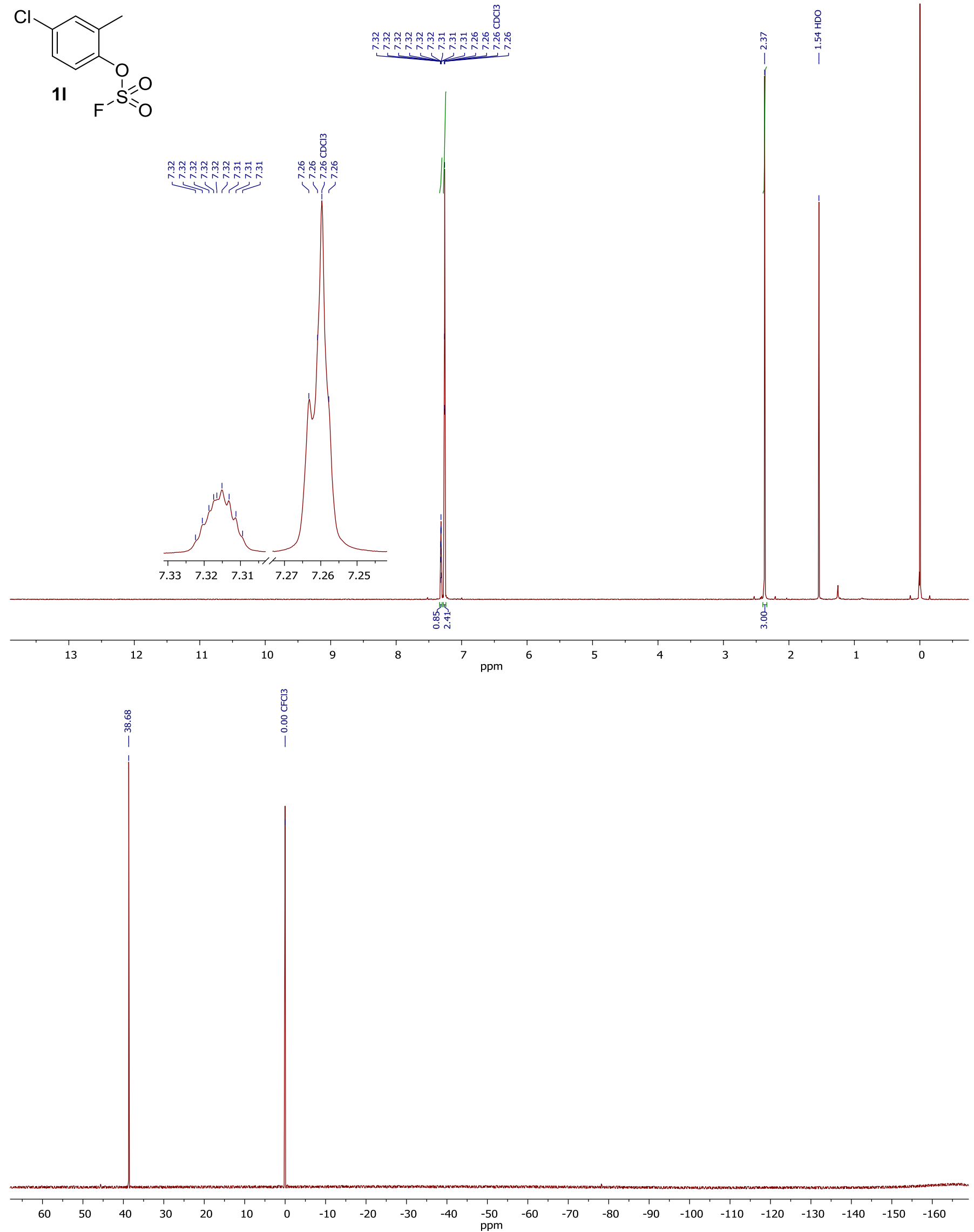

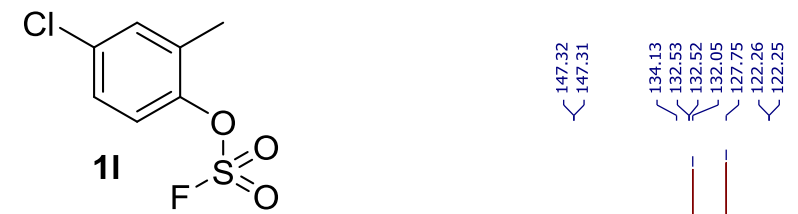

बूล

1) $F^{-S} \stackrel{1}{=}=0$
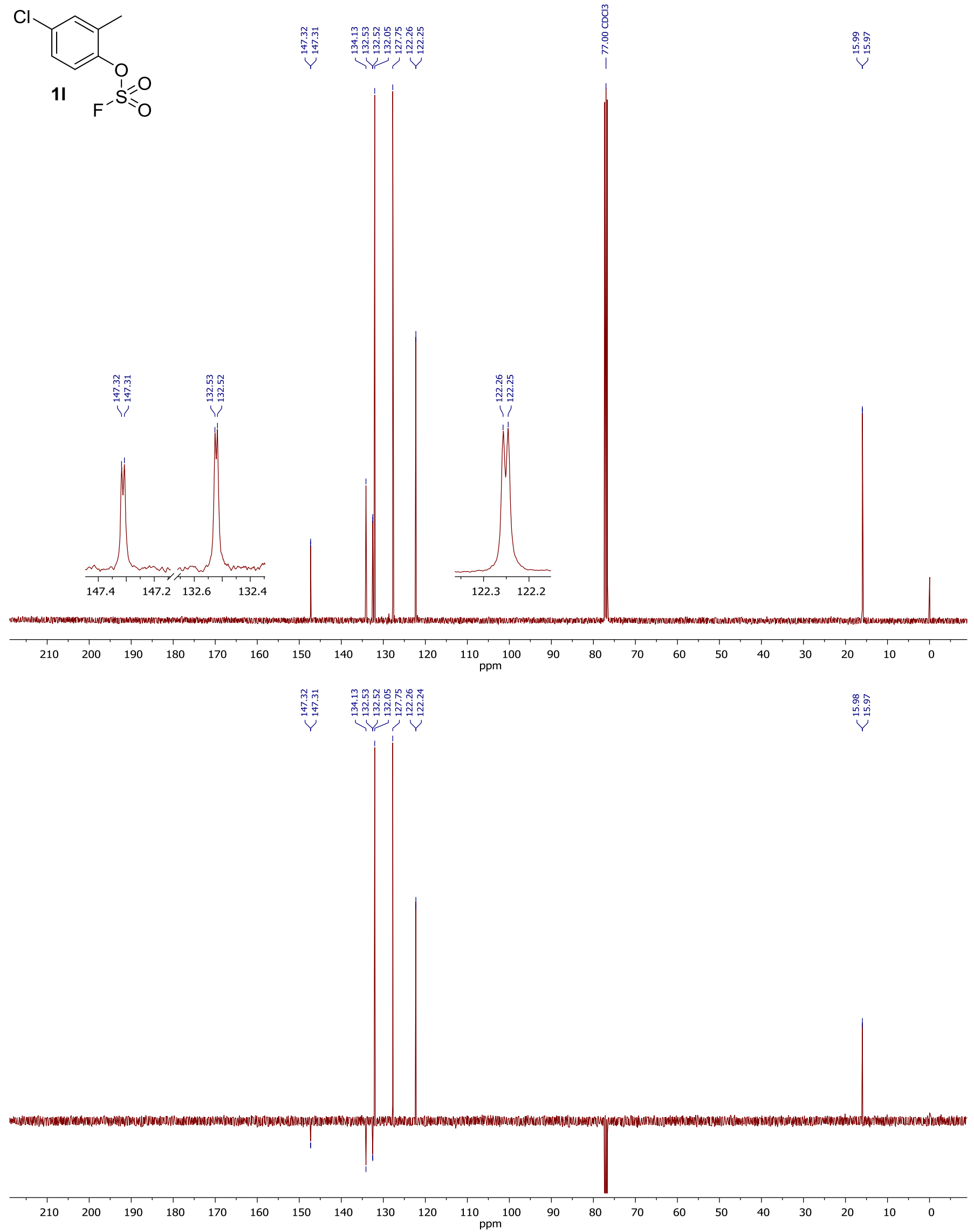

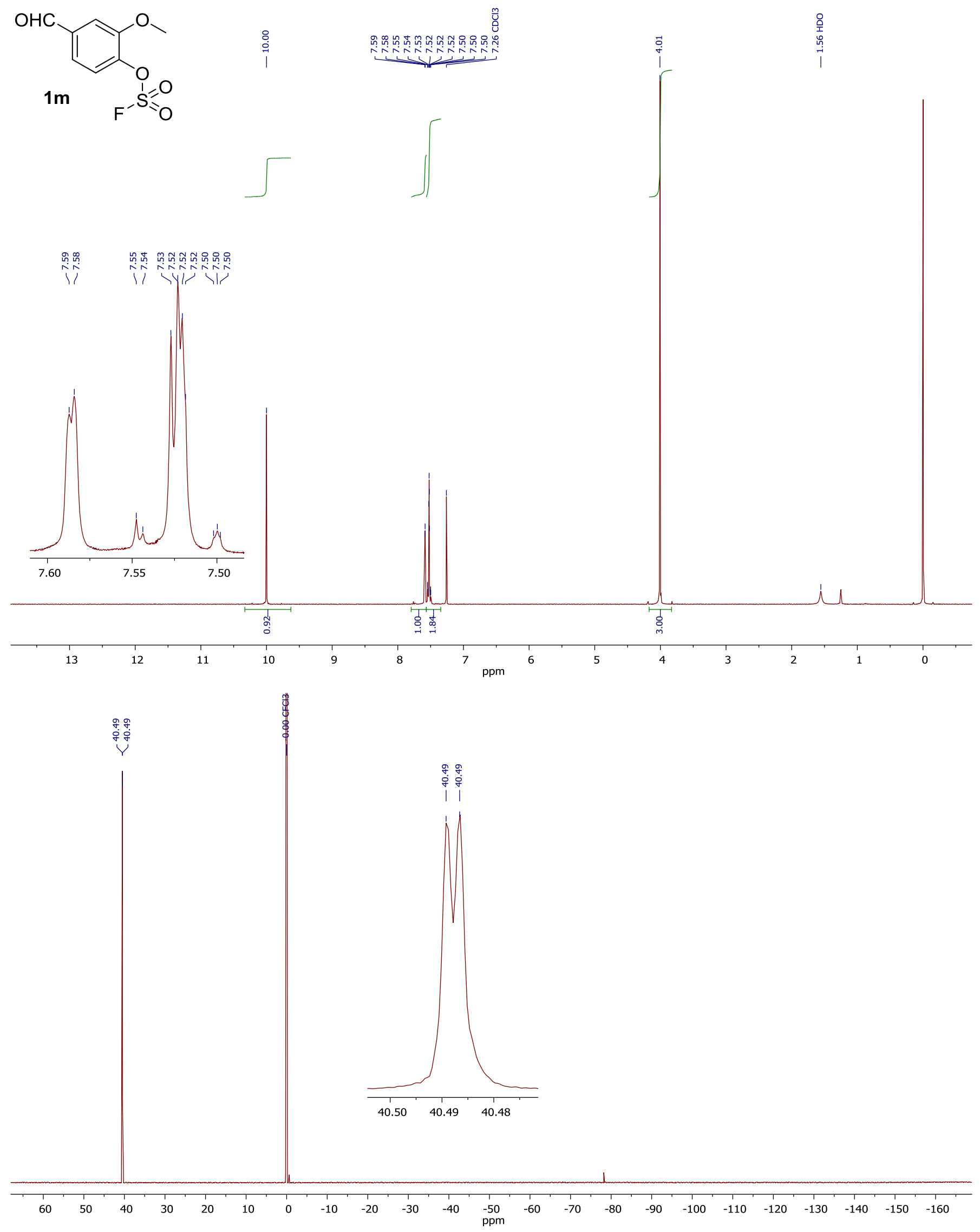


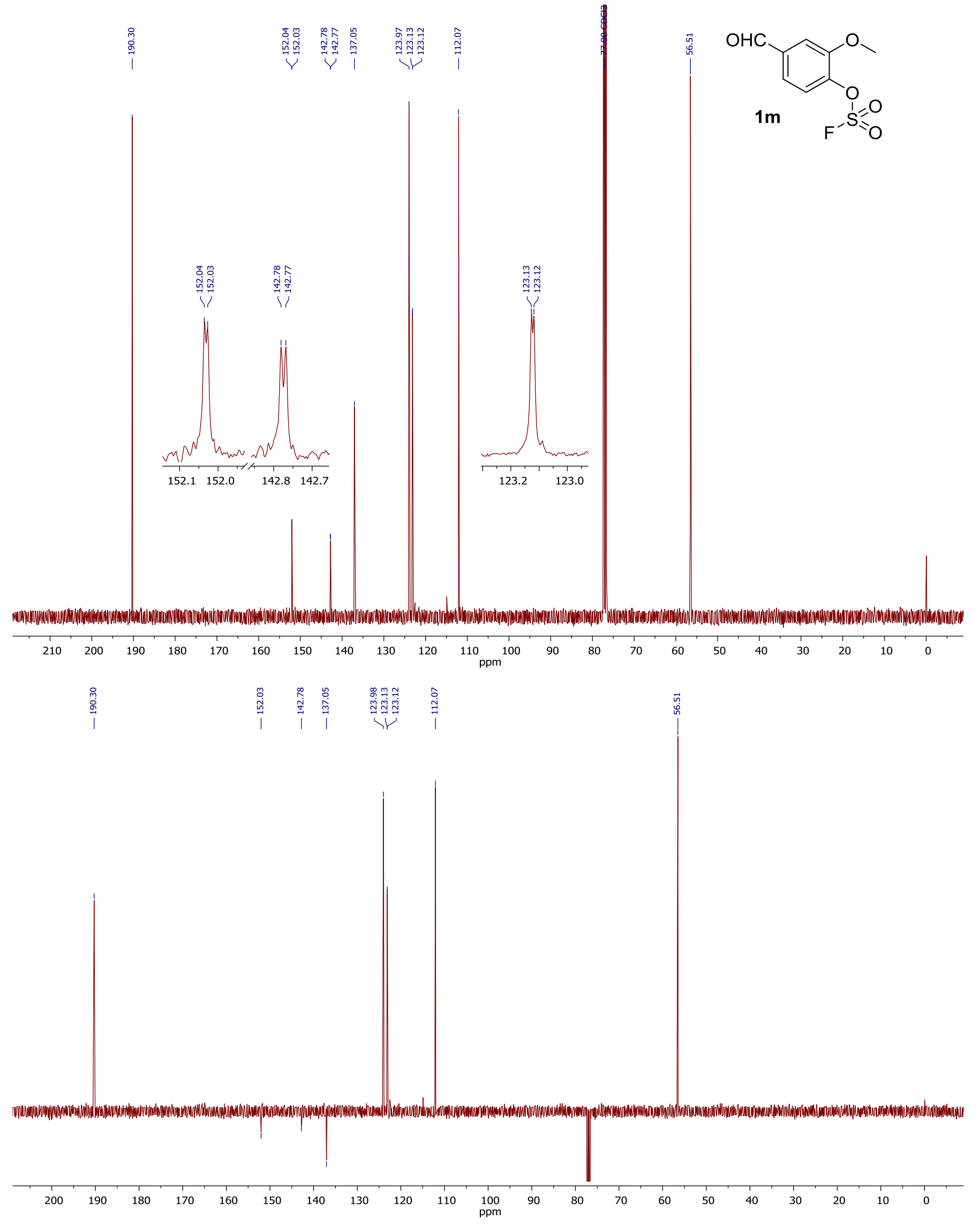


<smiles>O=Cc1ccccc1OS(=O)(=O)F</smiles>

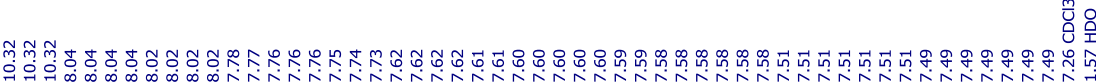

1n $F^{-S}=0$
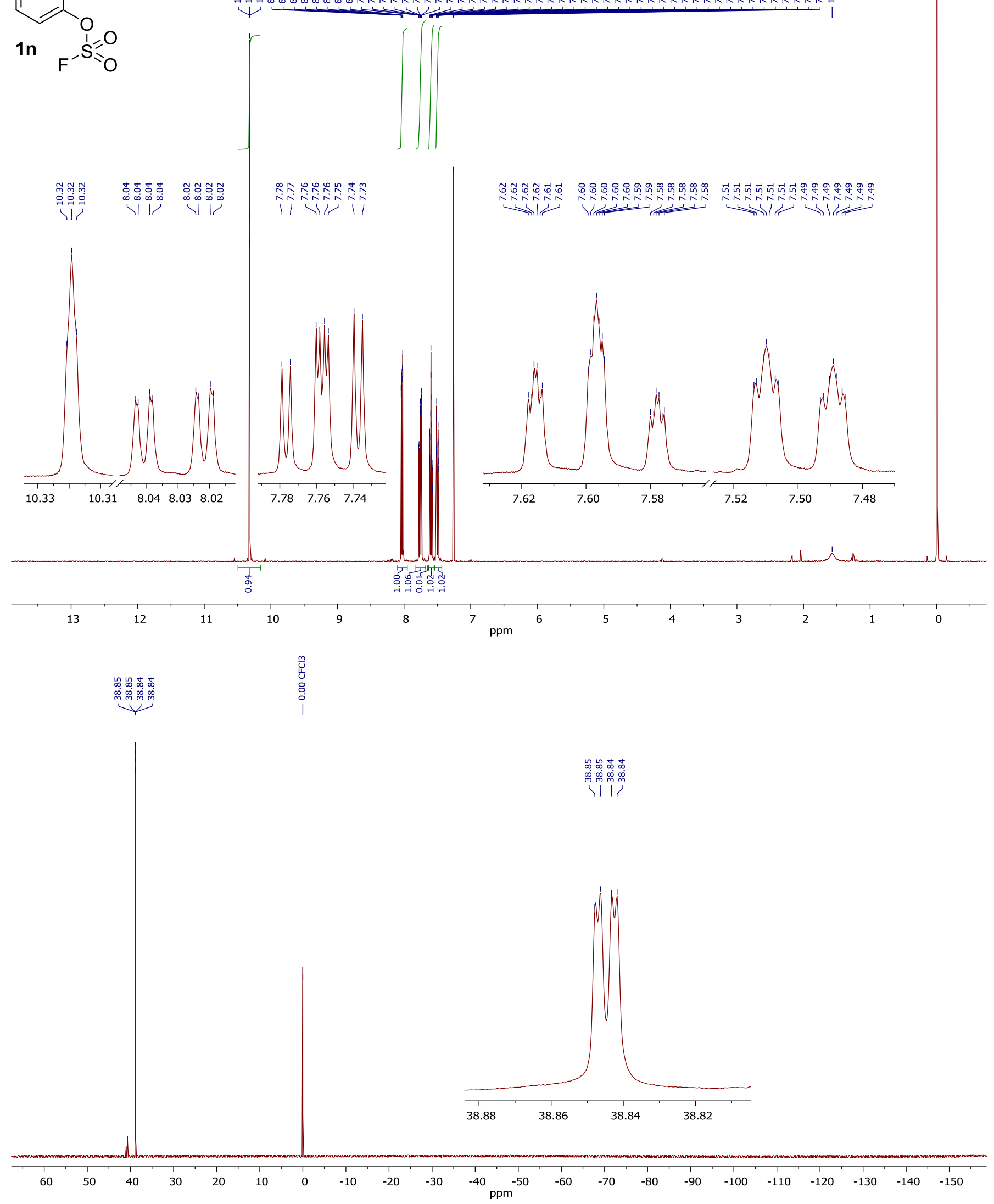


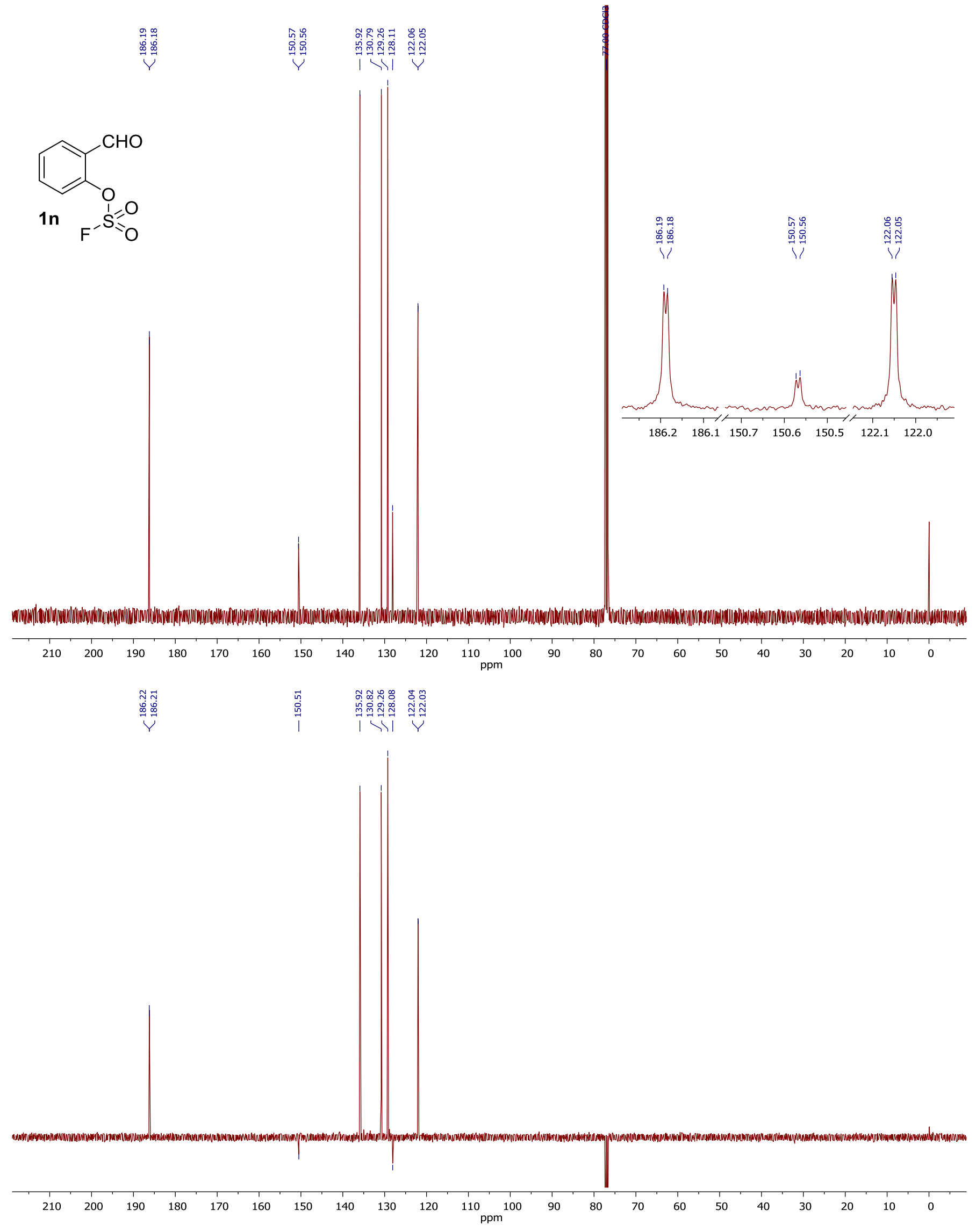



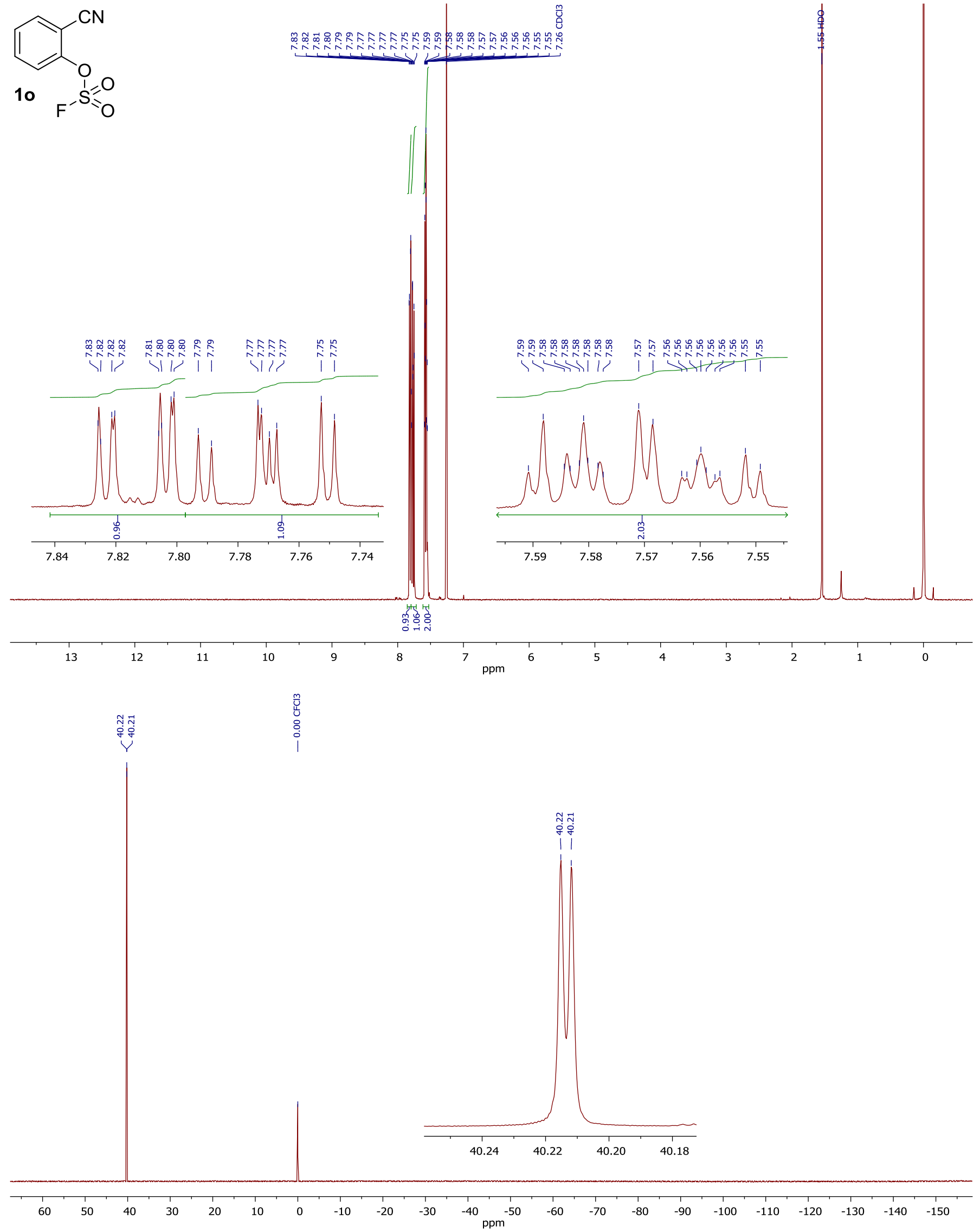

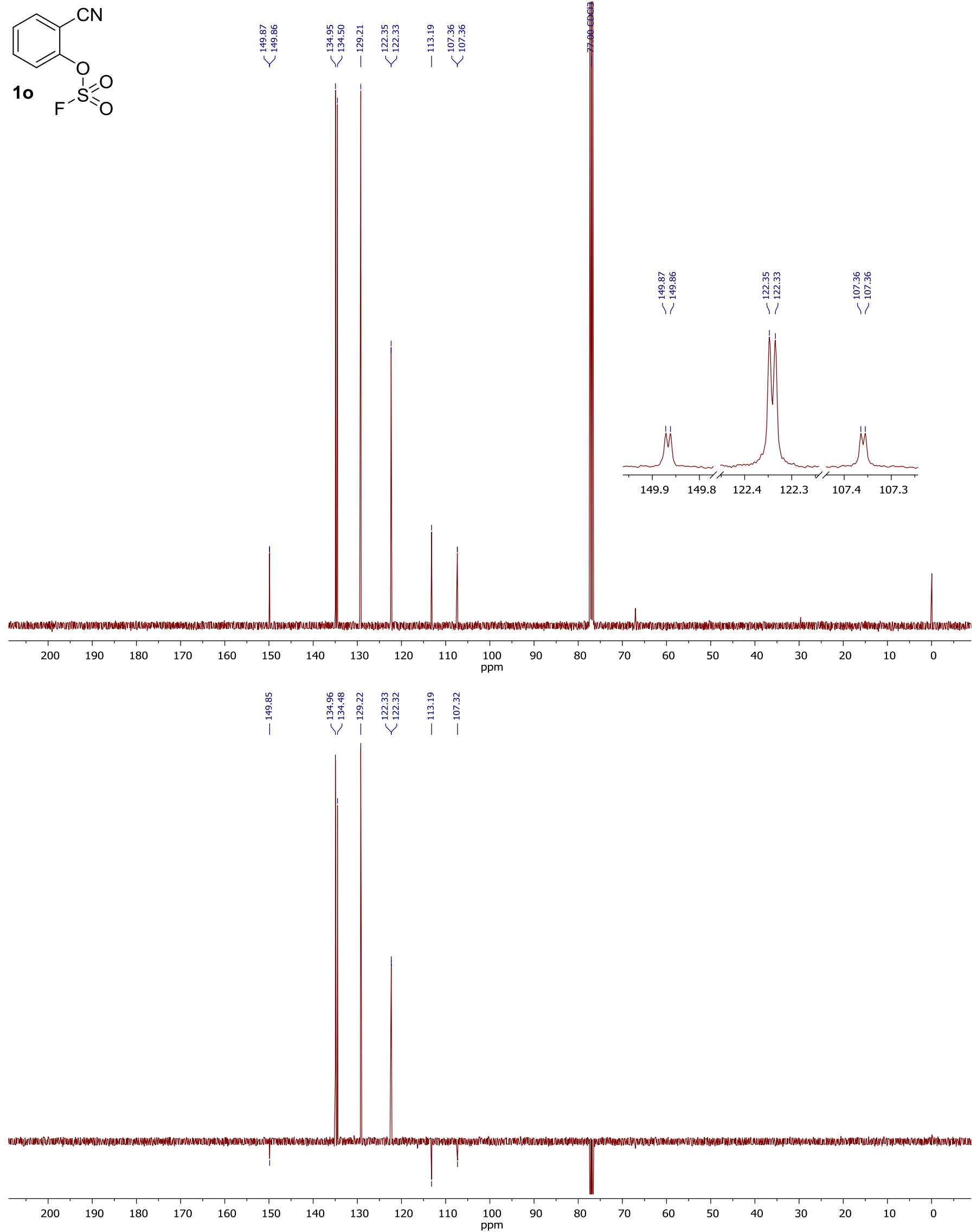

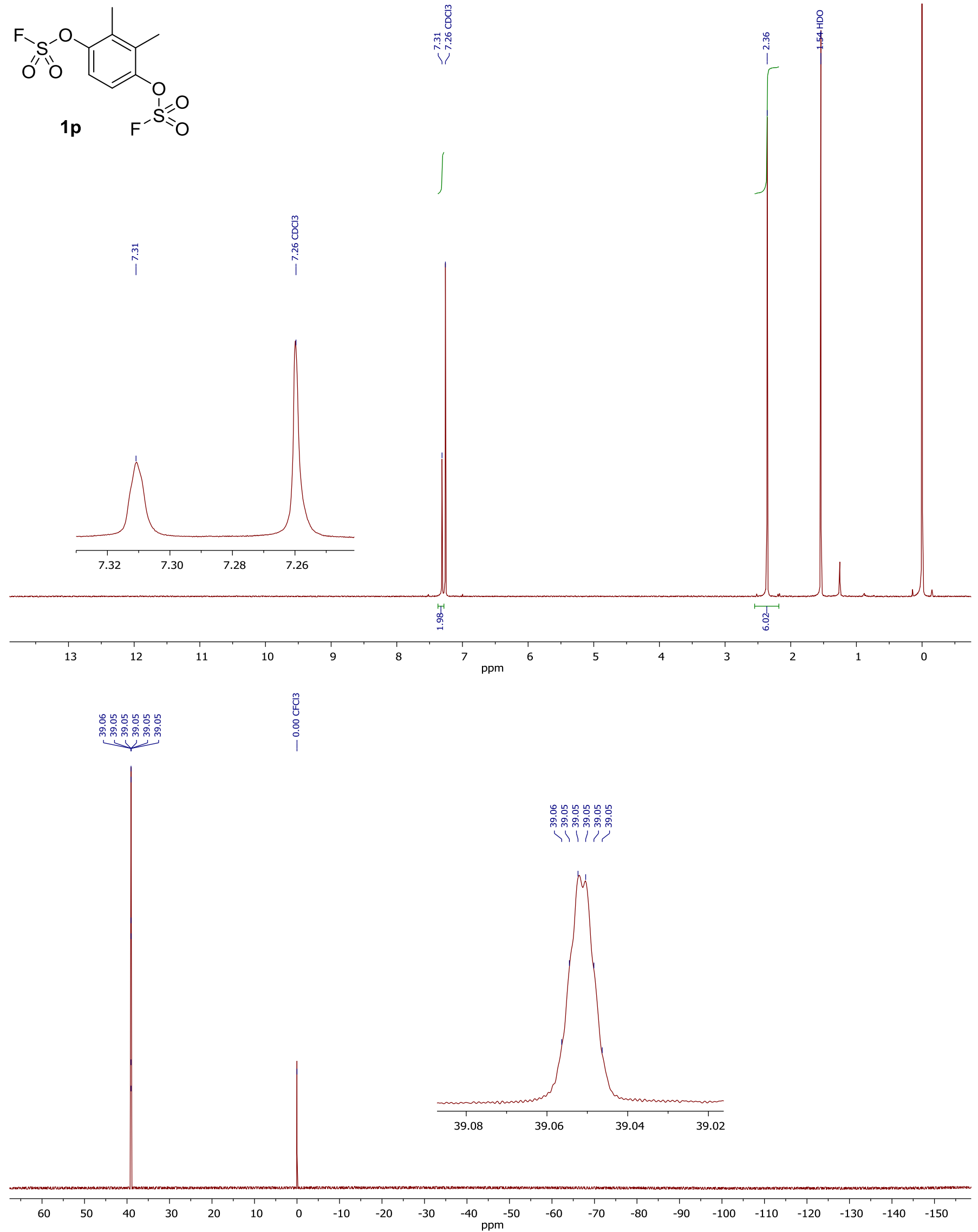

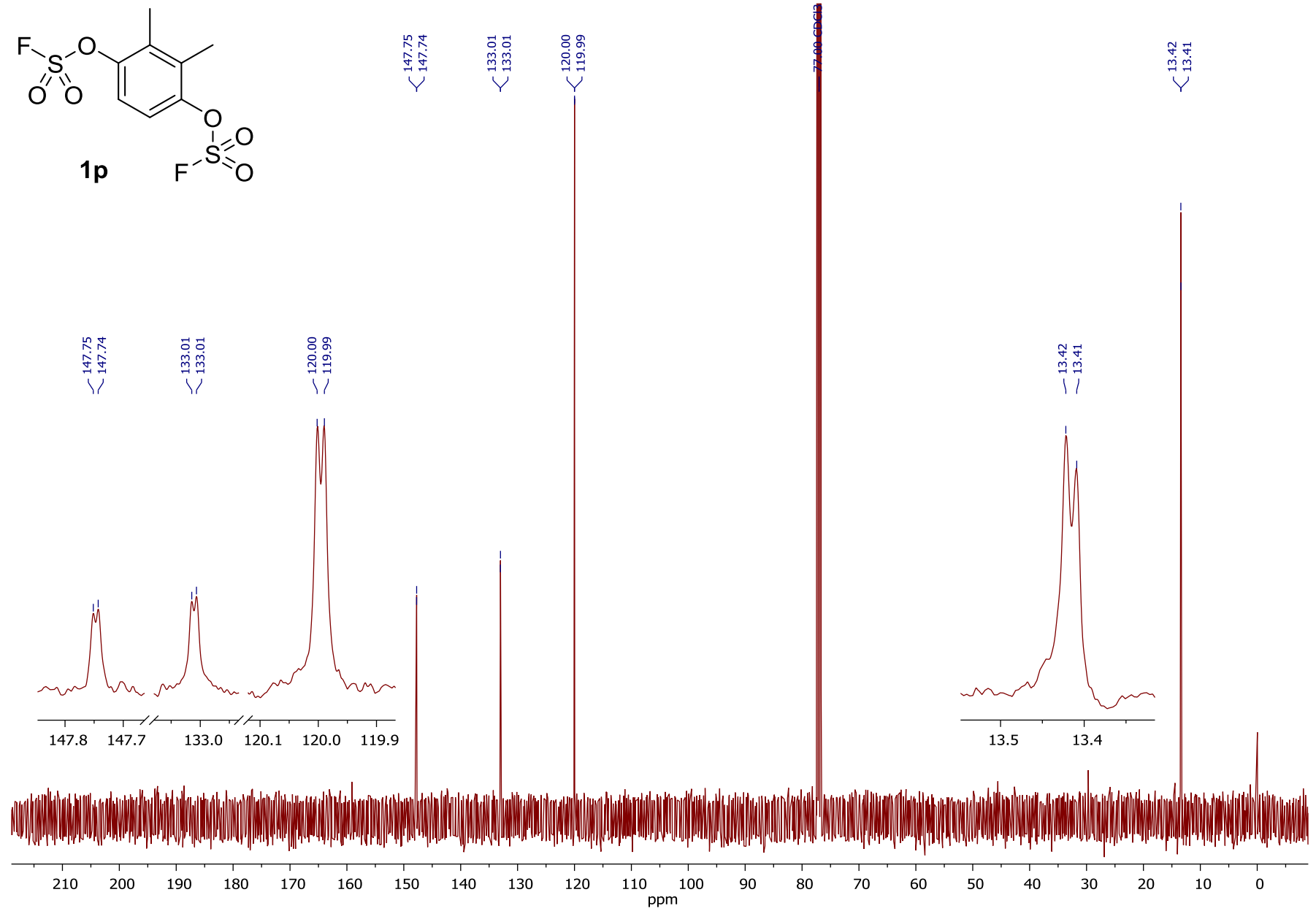

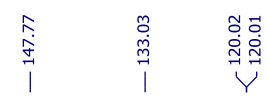
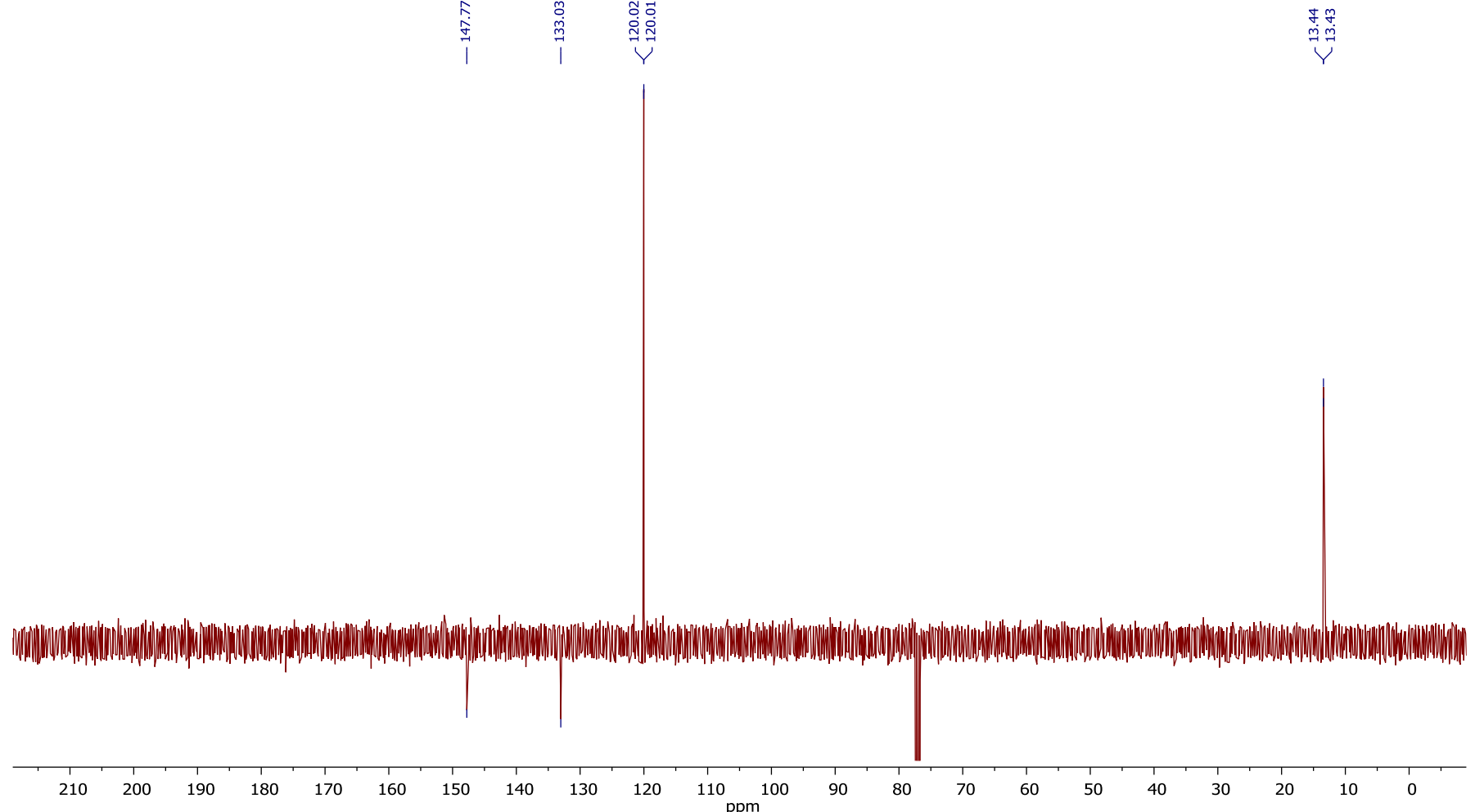
<smiles>CCC(=O)c1ccc(OS(=O)(=O)F)cc1</smiles>
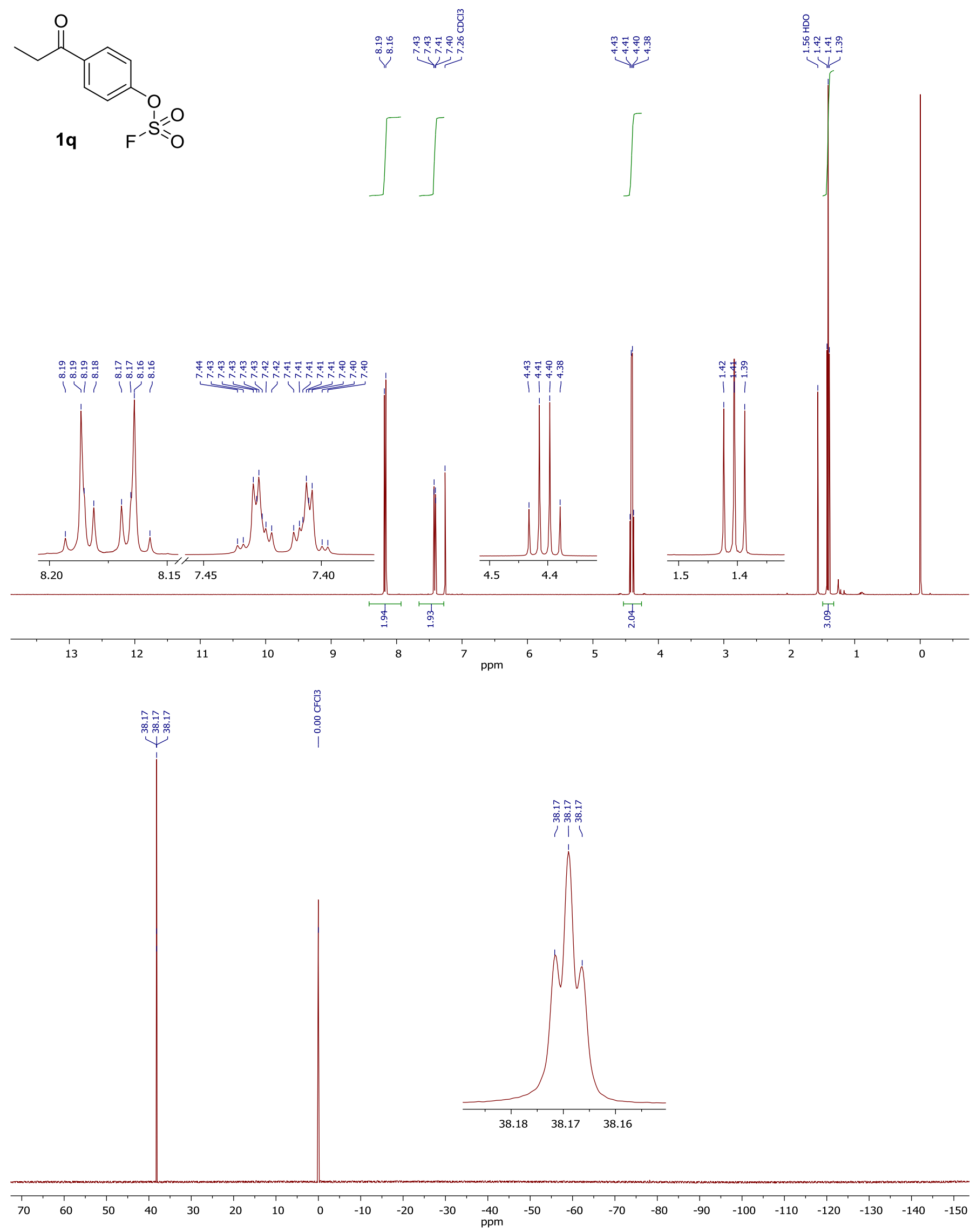

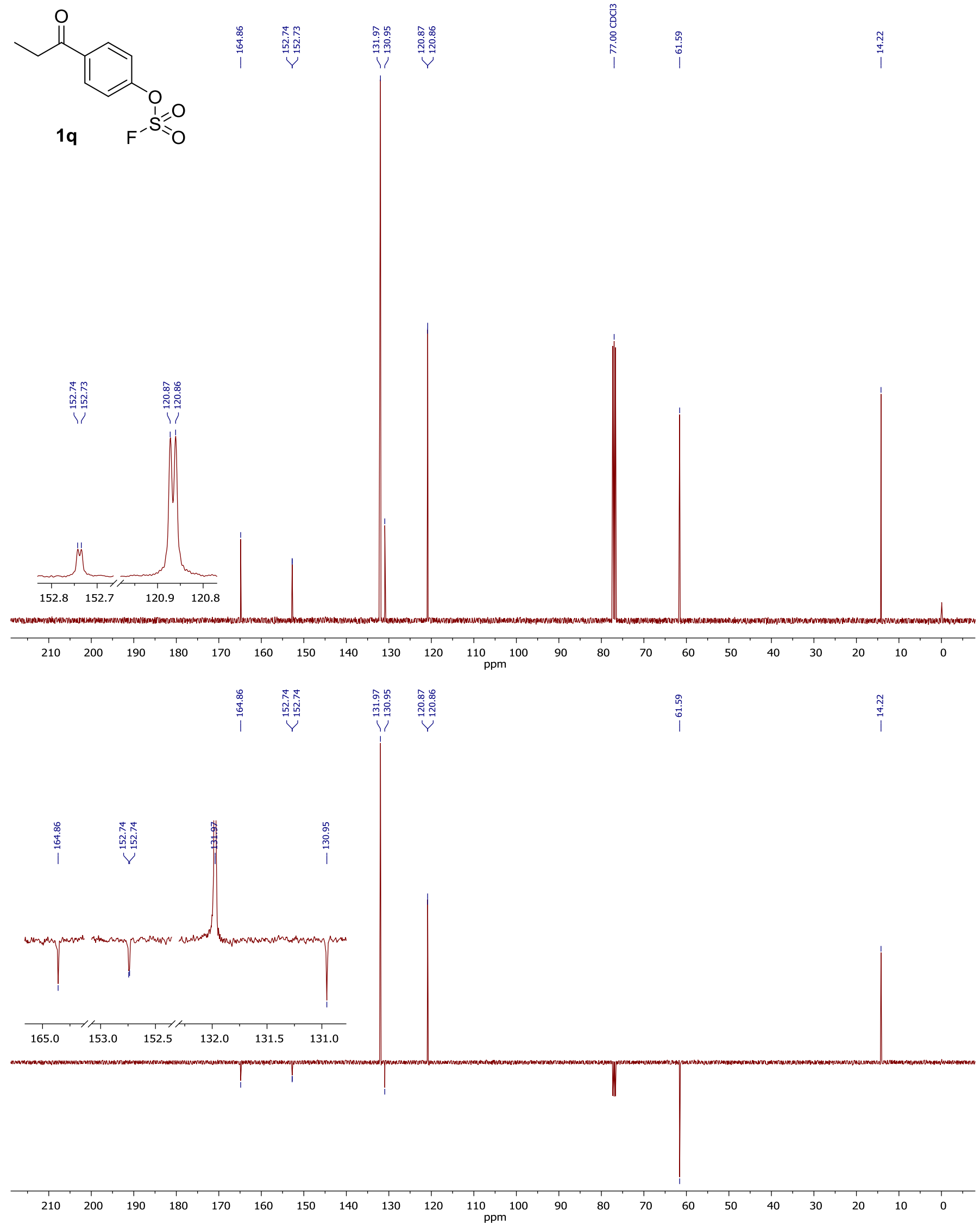

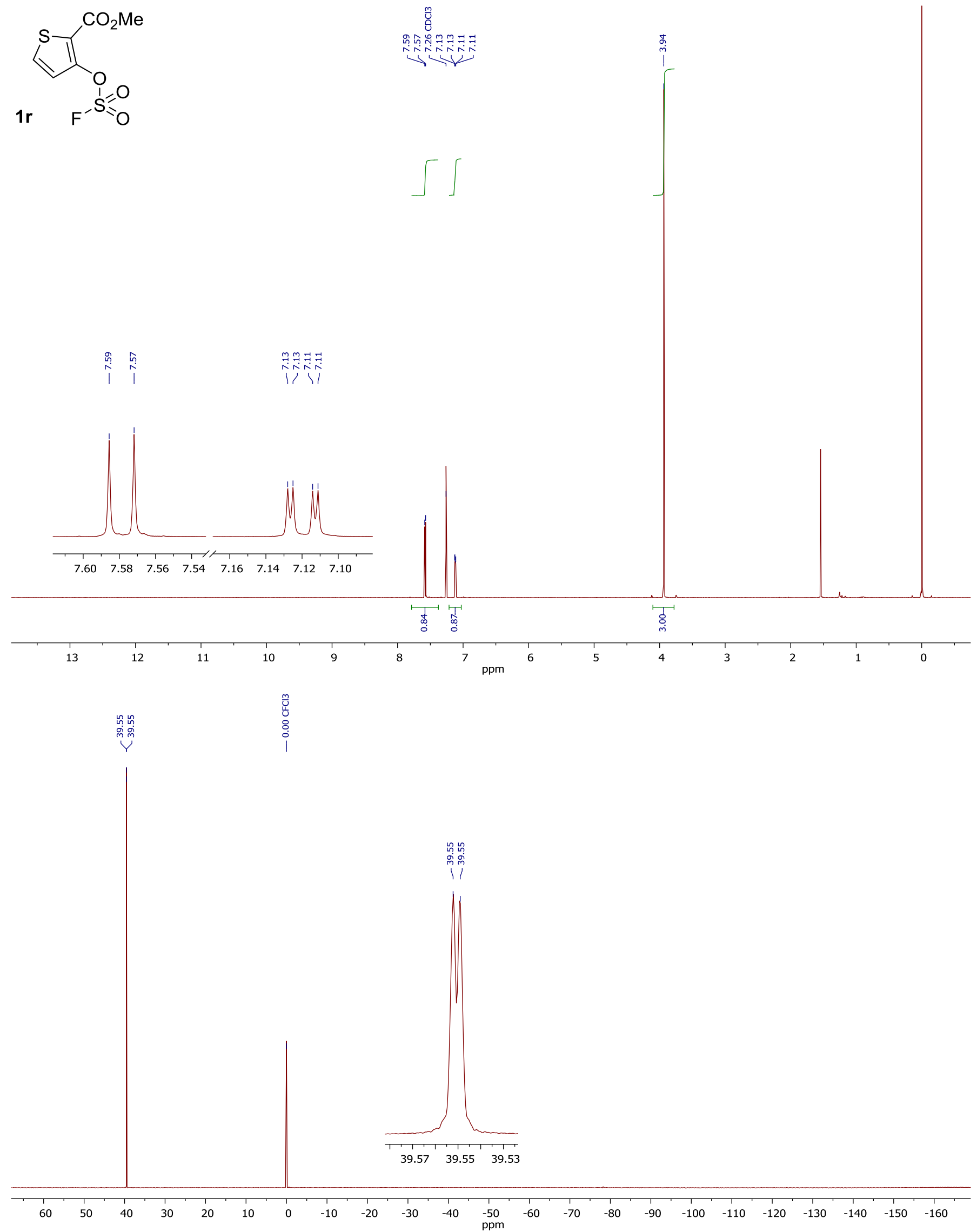


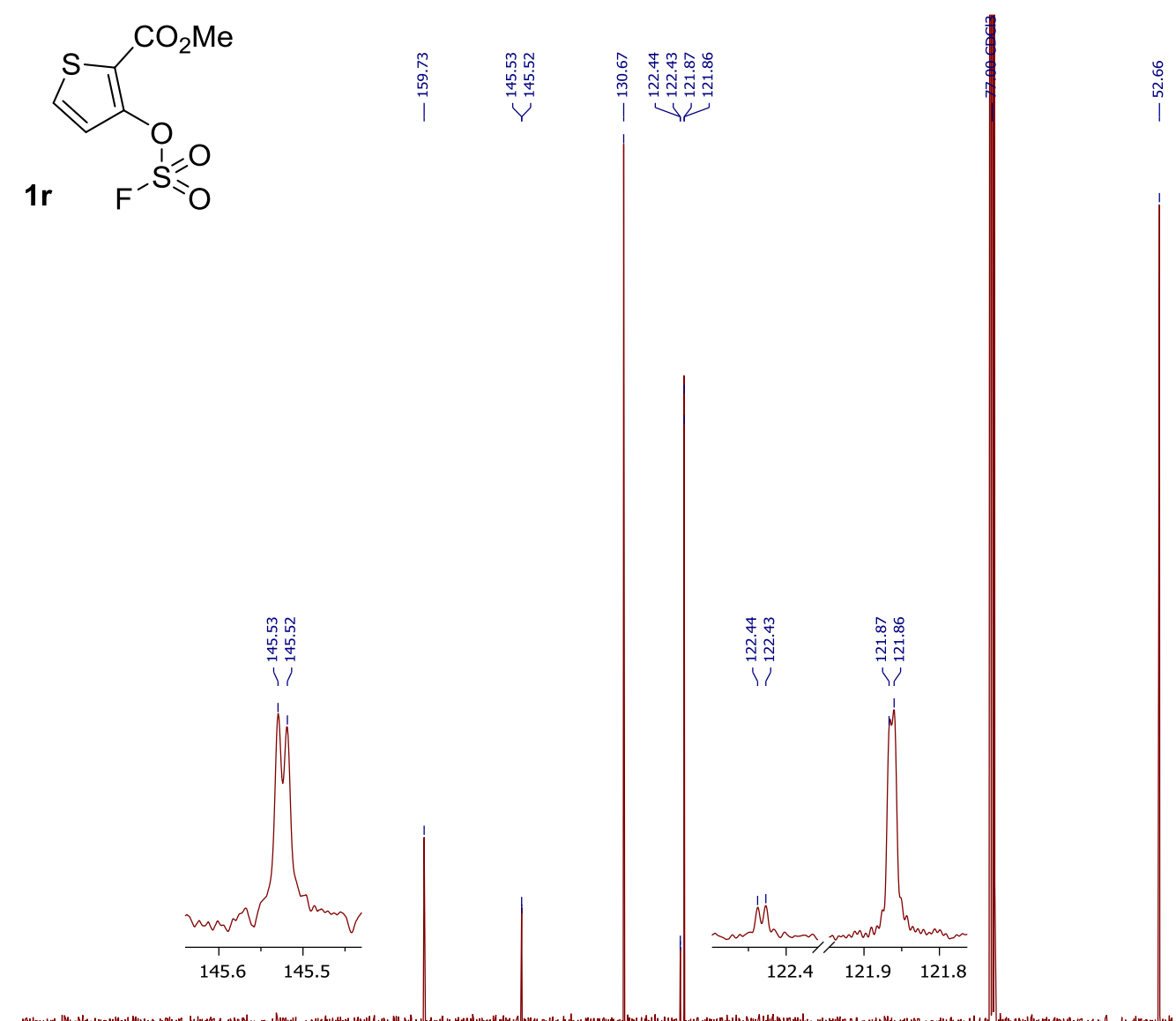

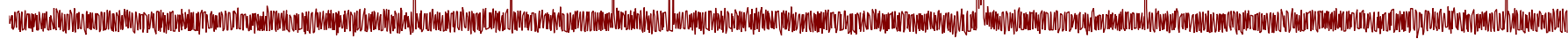
$\begin{array}{llllllllllllllllllllll}210 & 200 & 190 & 180 & 170 & 160 & 150 & 140 & 130 & 120 & 110 & 100 & 90 & 80 & 70 & 60 & 50 & 40 & 30 & 20 & 10 & 0\end{array}$

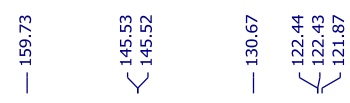

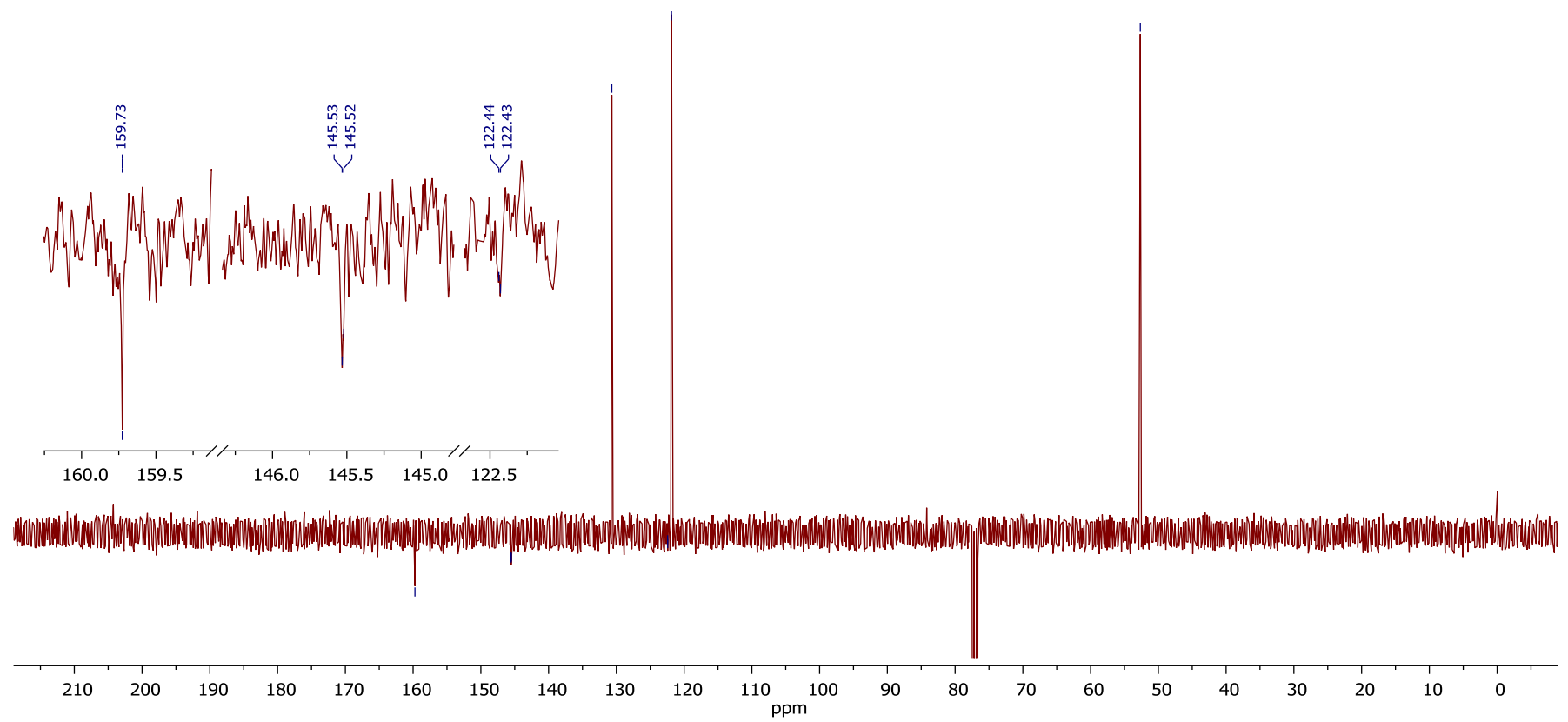



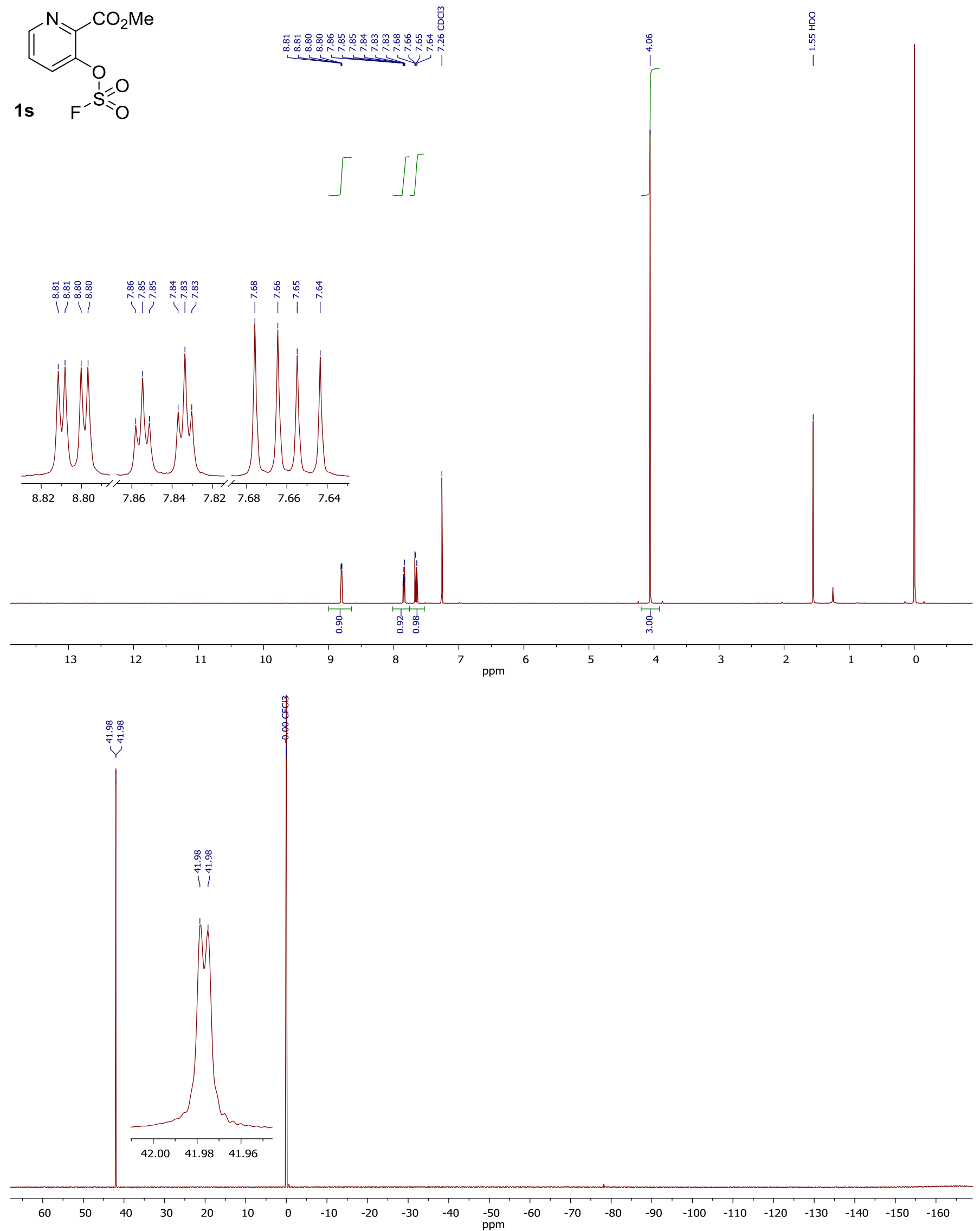

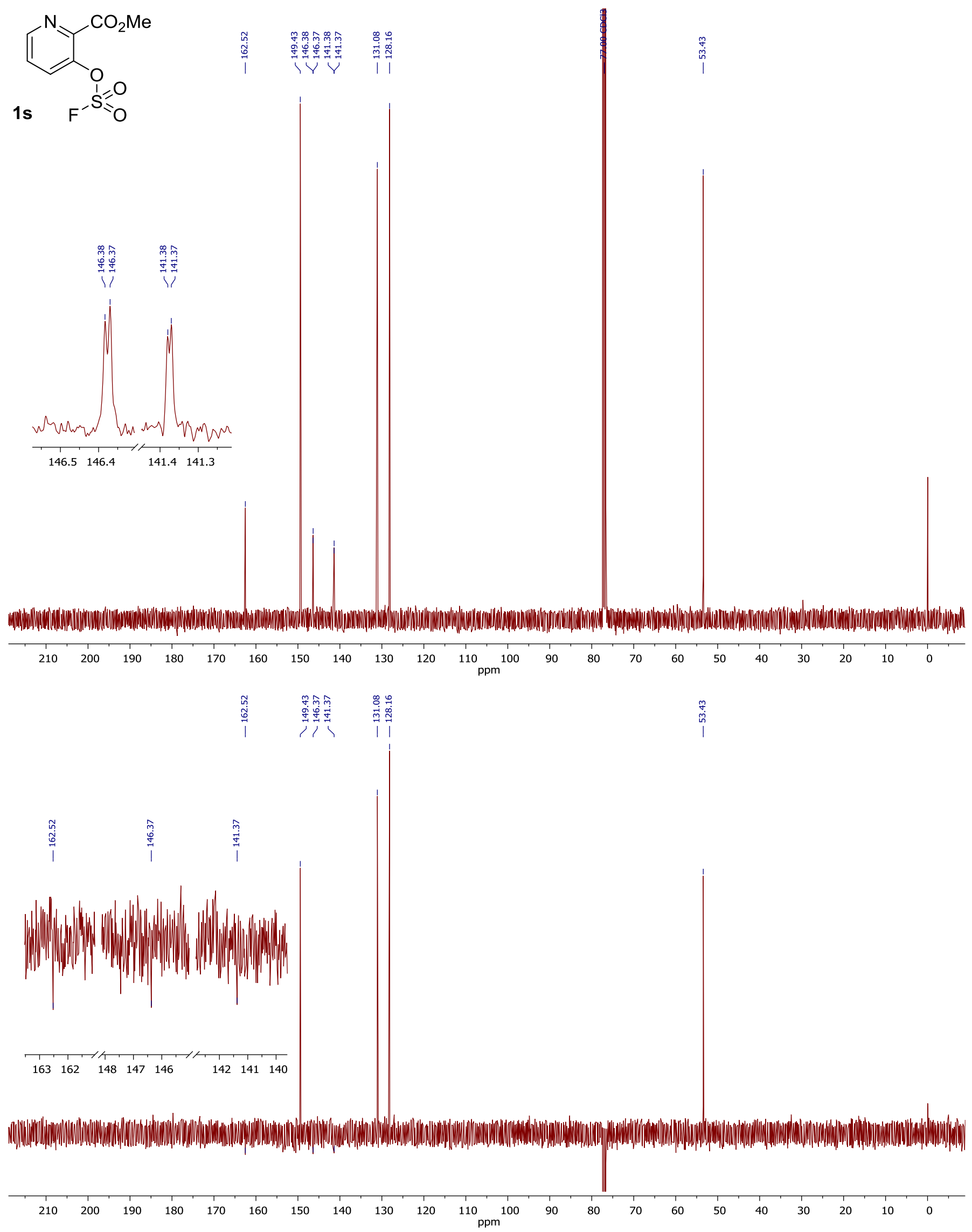


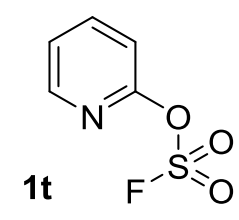

m.

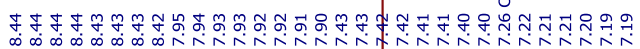

(1)

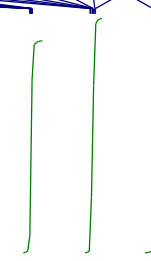

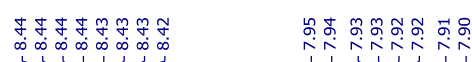

जोl

।रोा। ।र
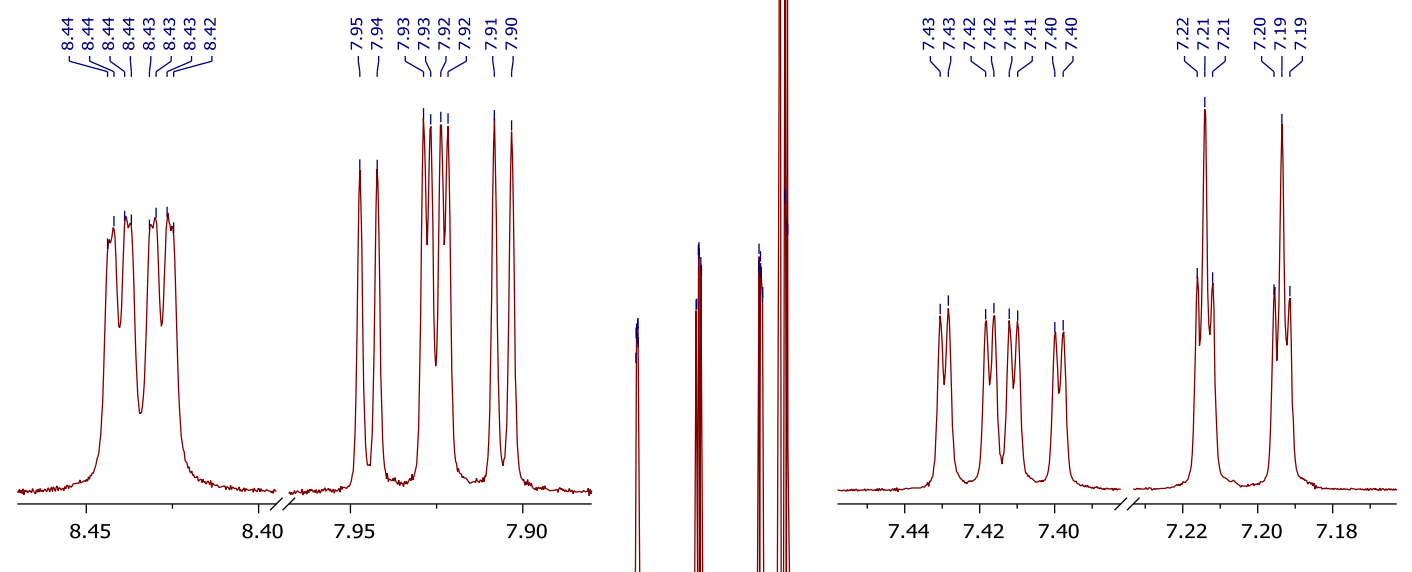

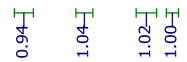

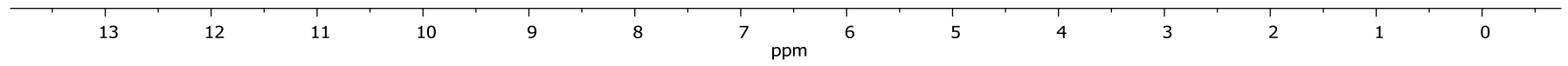

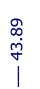

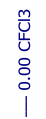
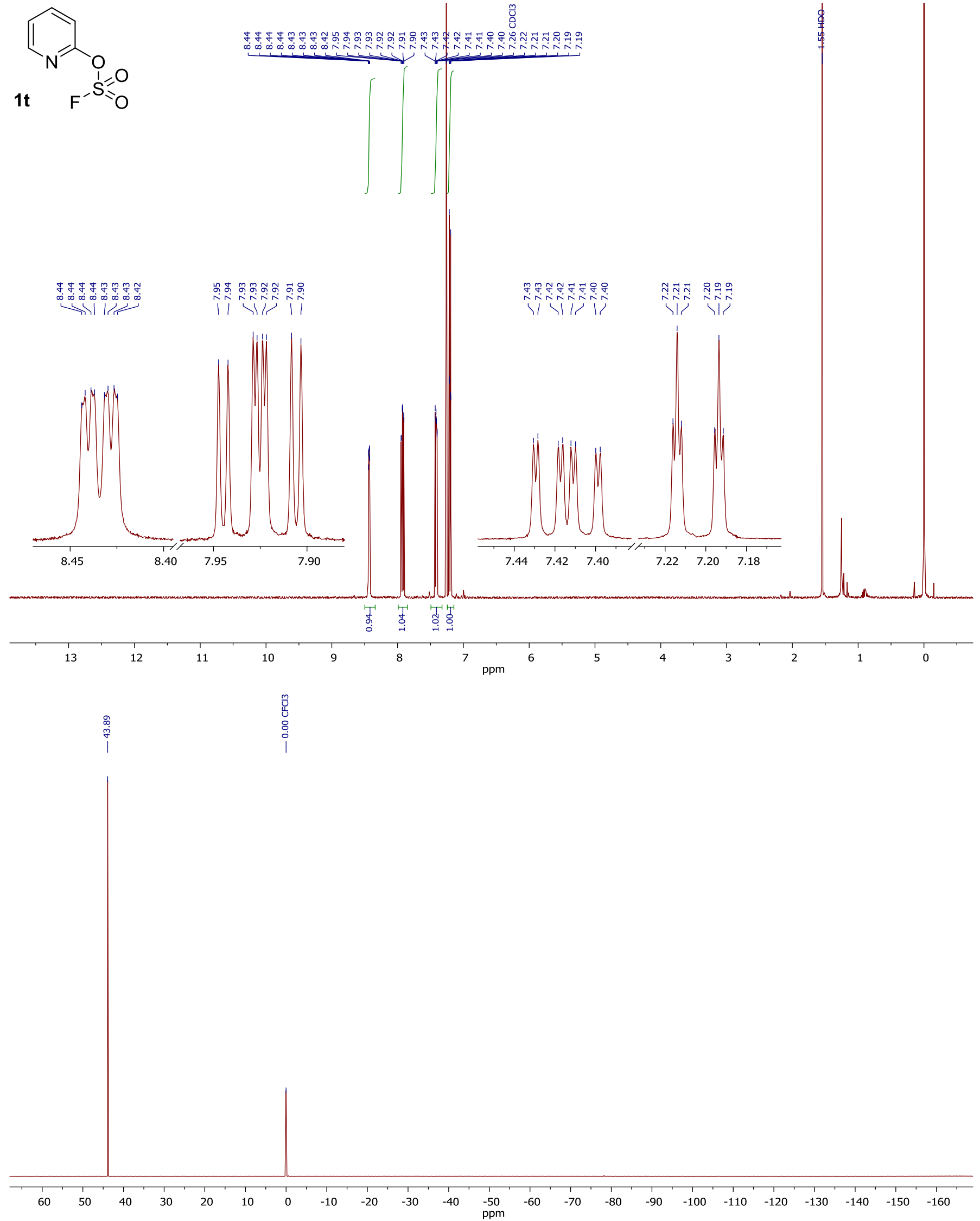


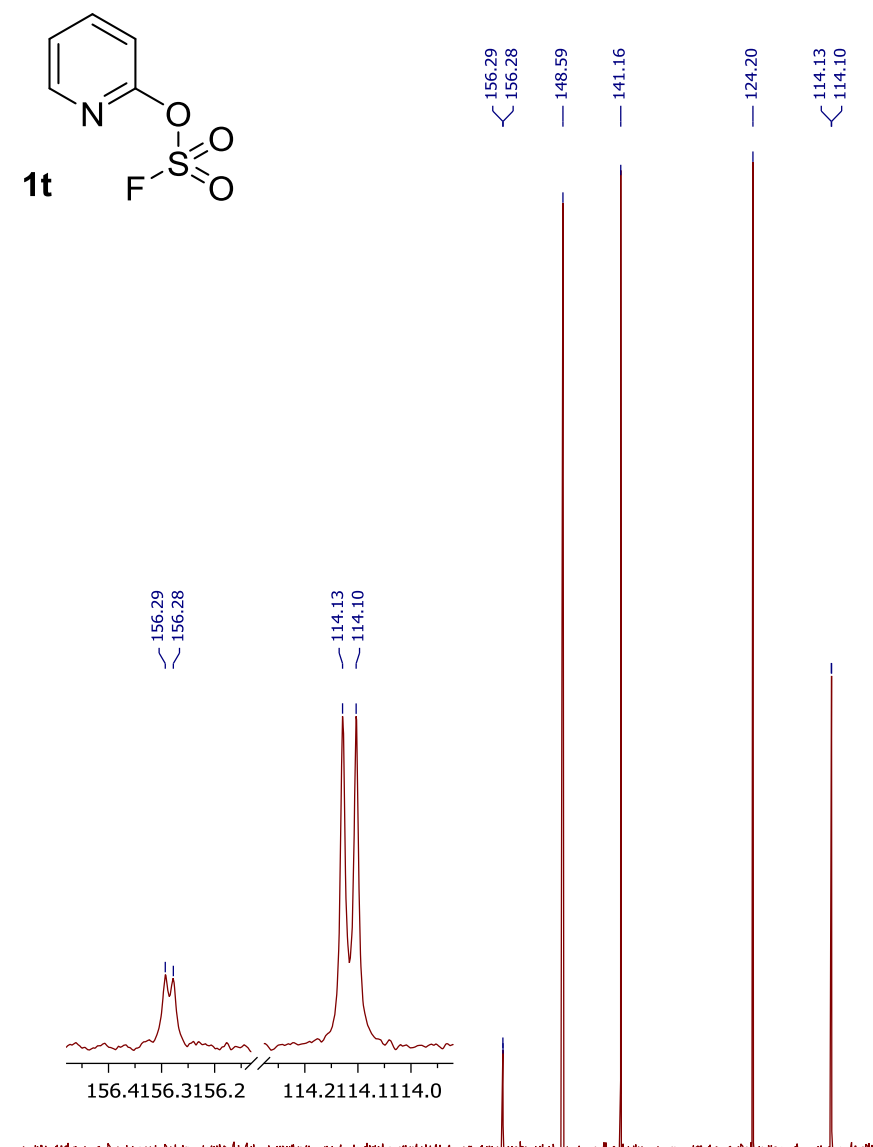

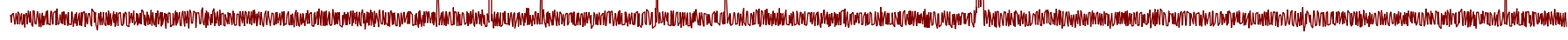

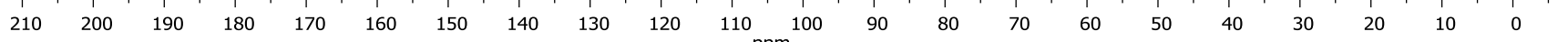

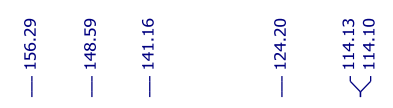

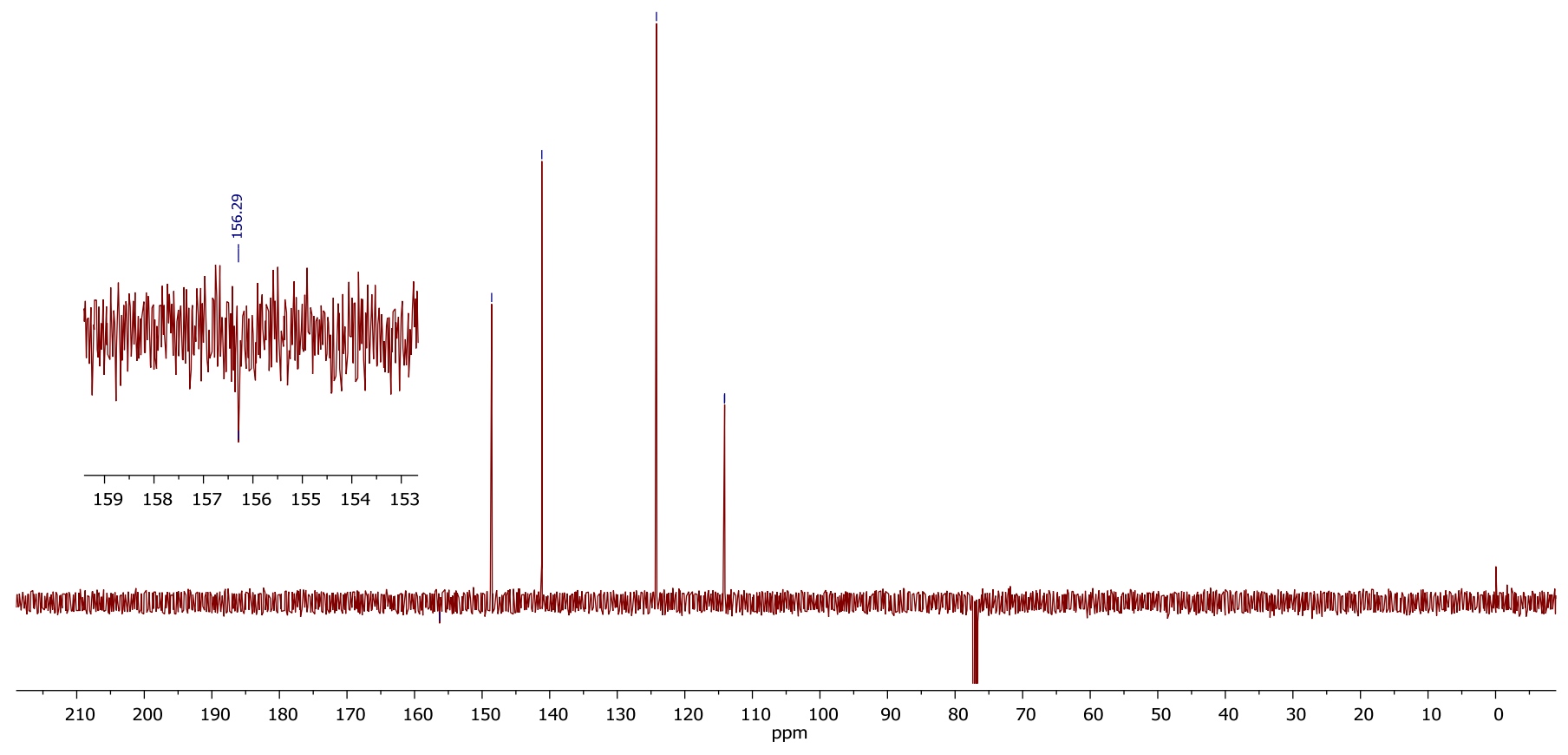



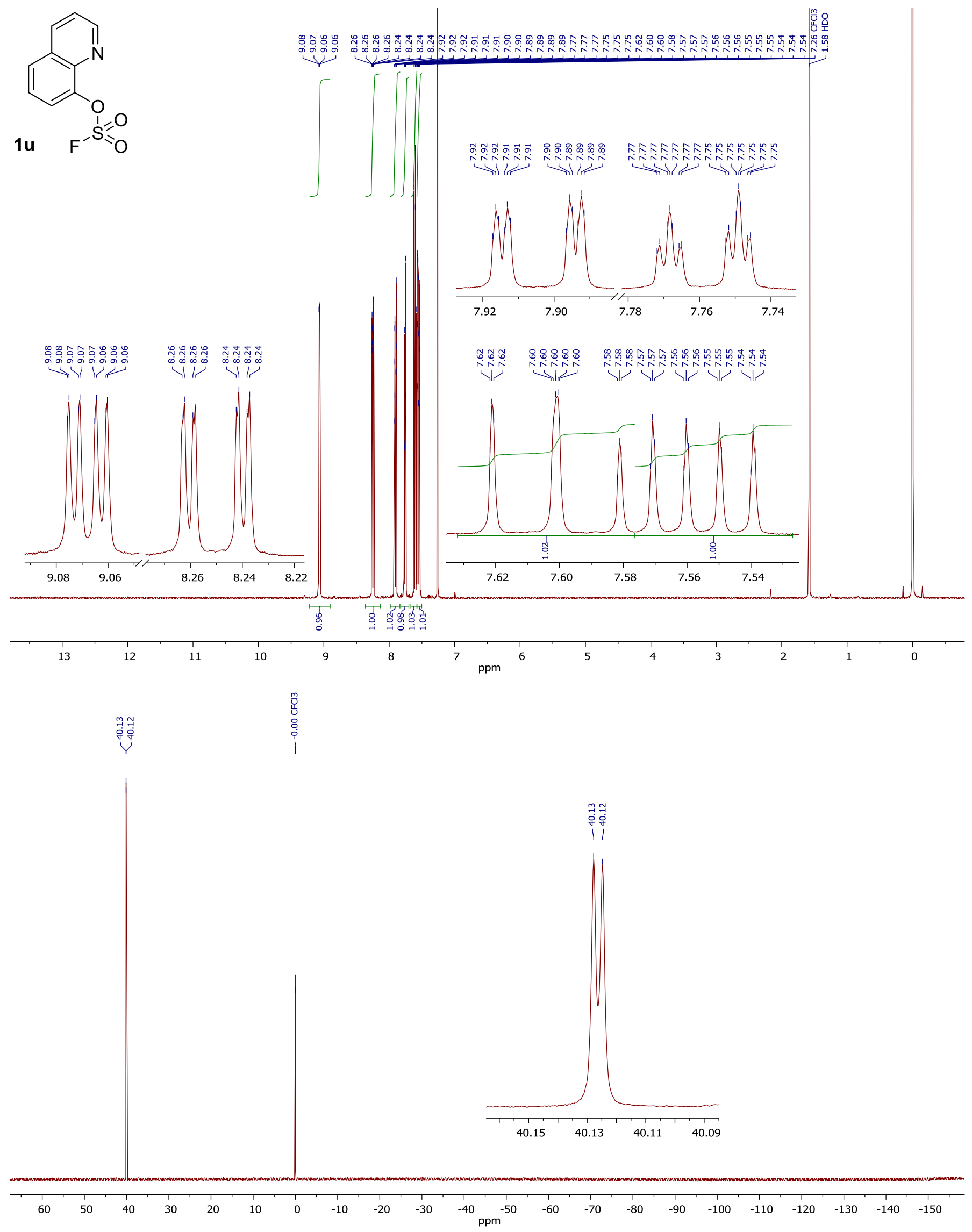

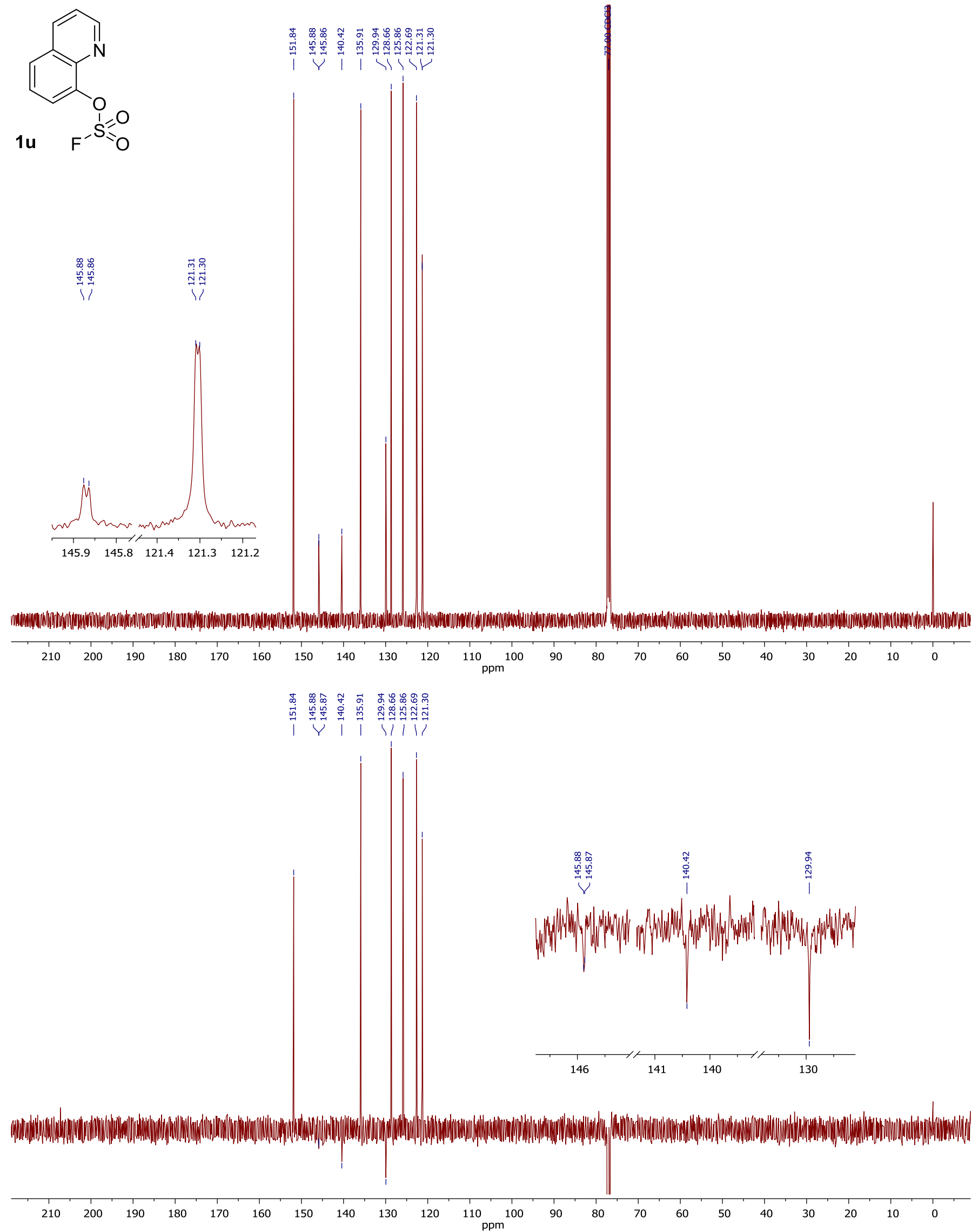
<smiles>COC(=O)c1ccccc1OS(=O)(=O)C(F)(F)F</smiles>

$5 a$

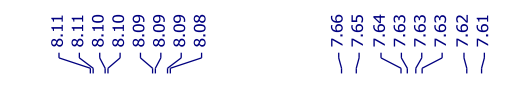

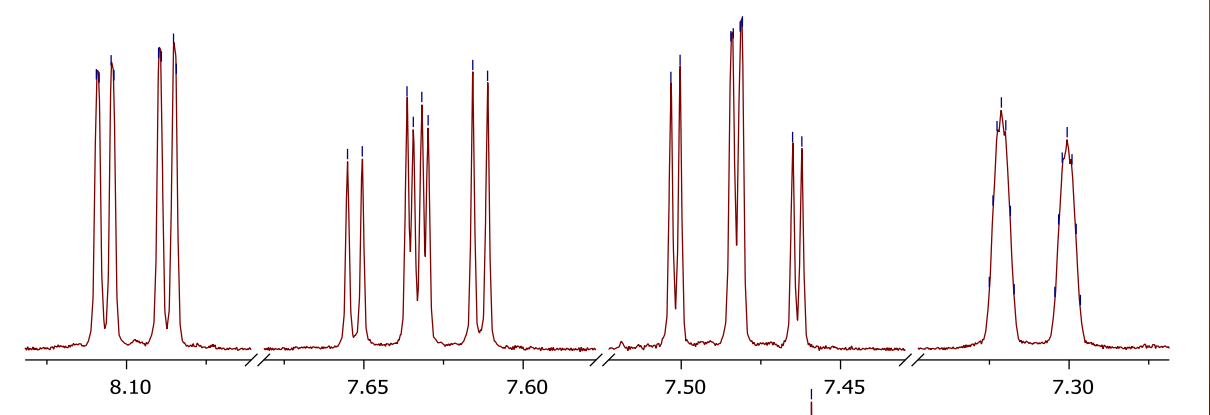
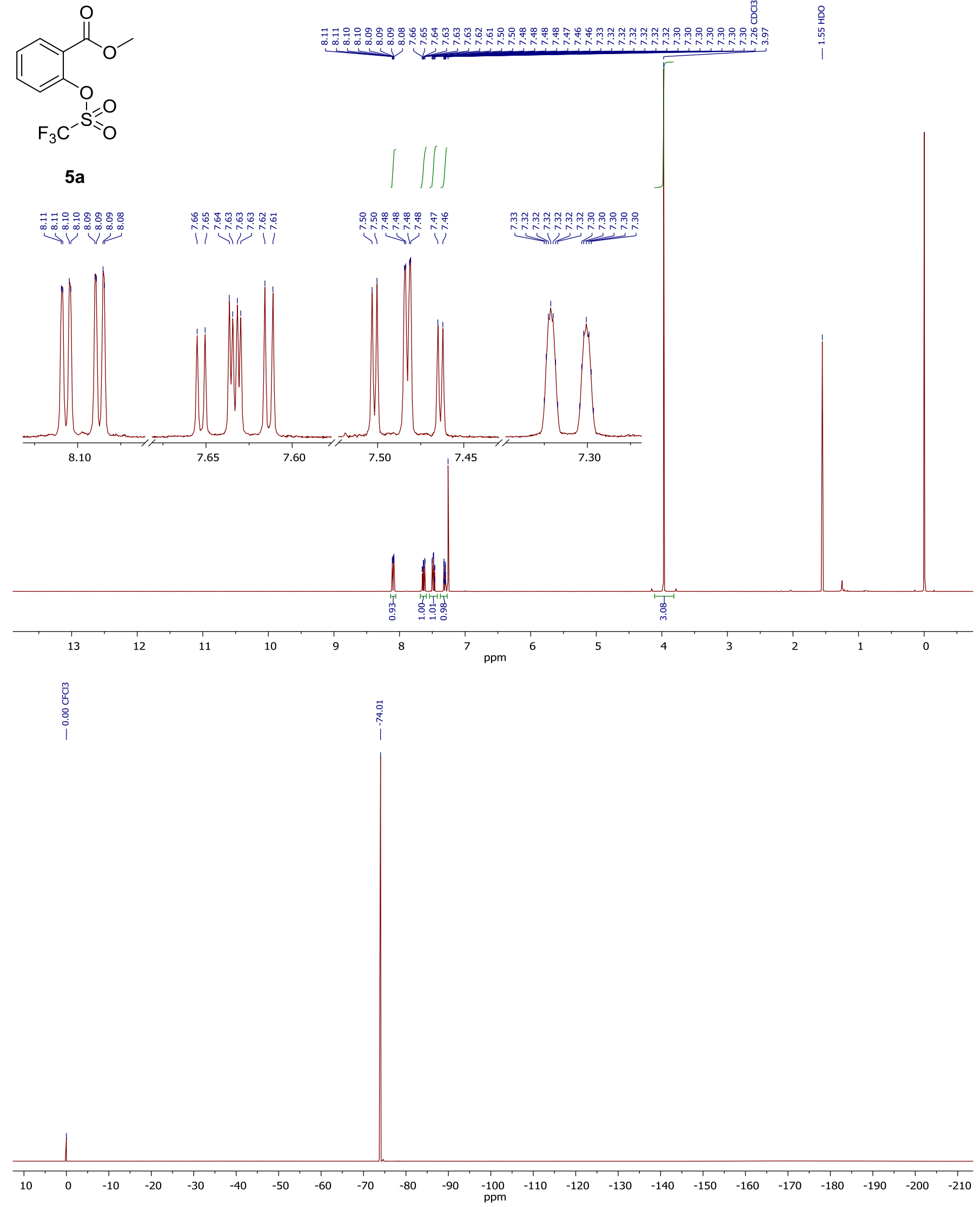

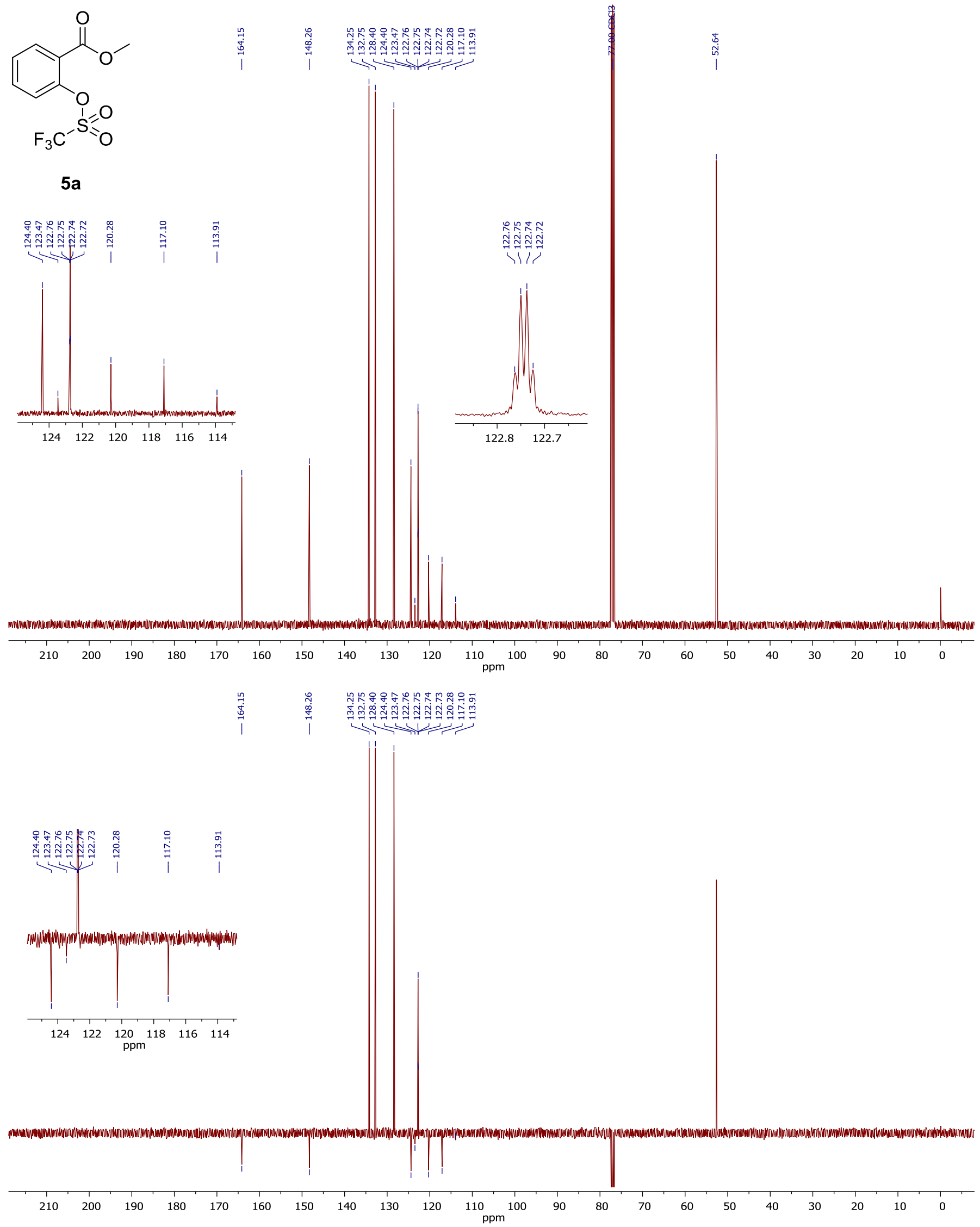
<smiles>COC(=O)c1ccccc1OS(C)(=O)=O</smiles>

$6 a$
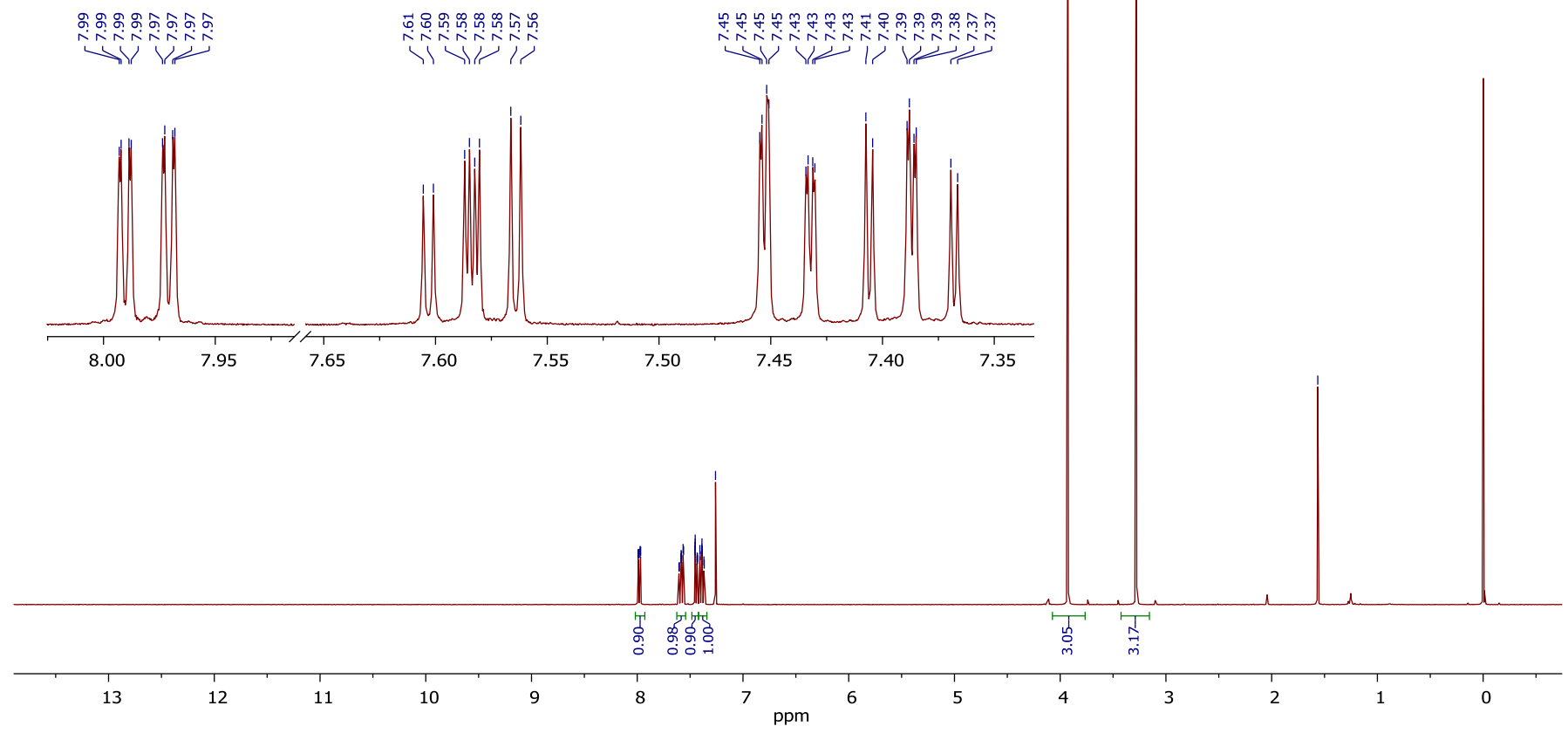


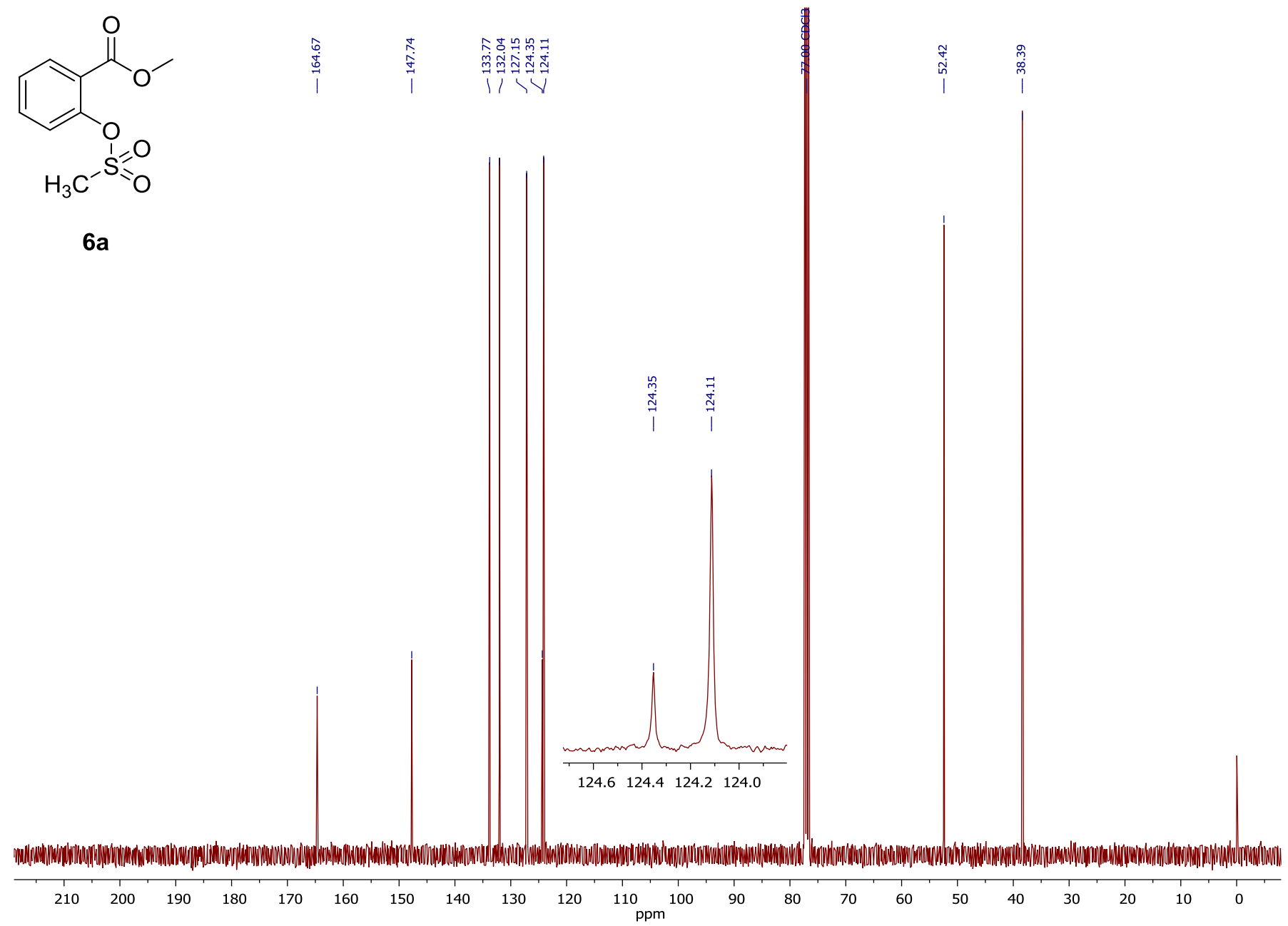




\subsection{Catellani products 4, 9 and 10}

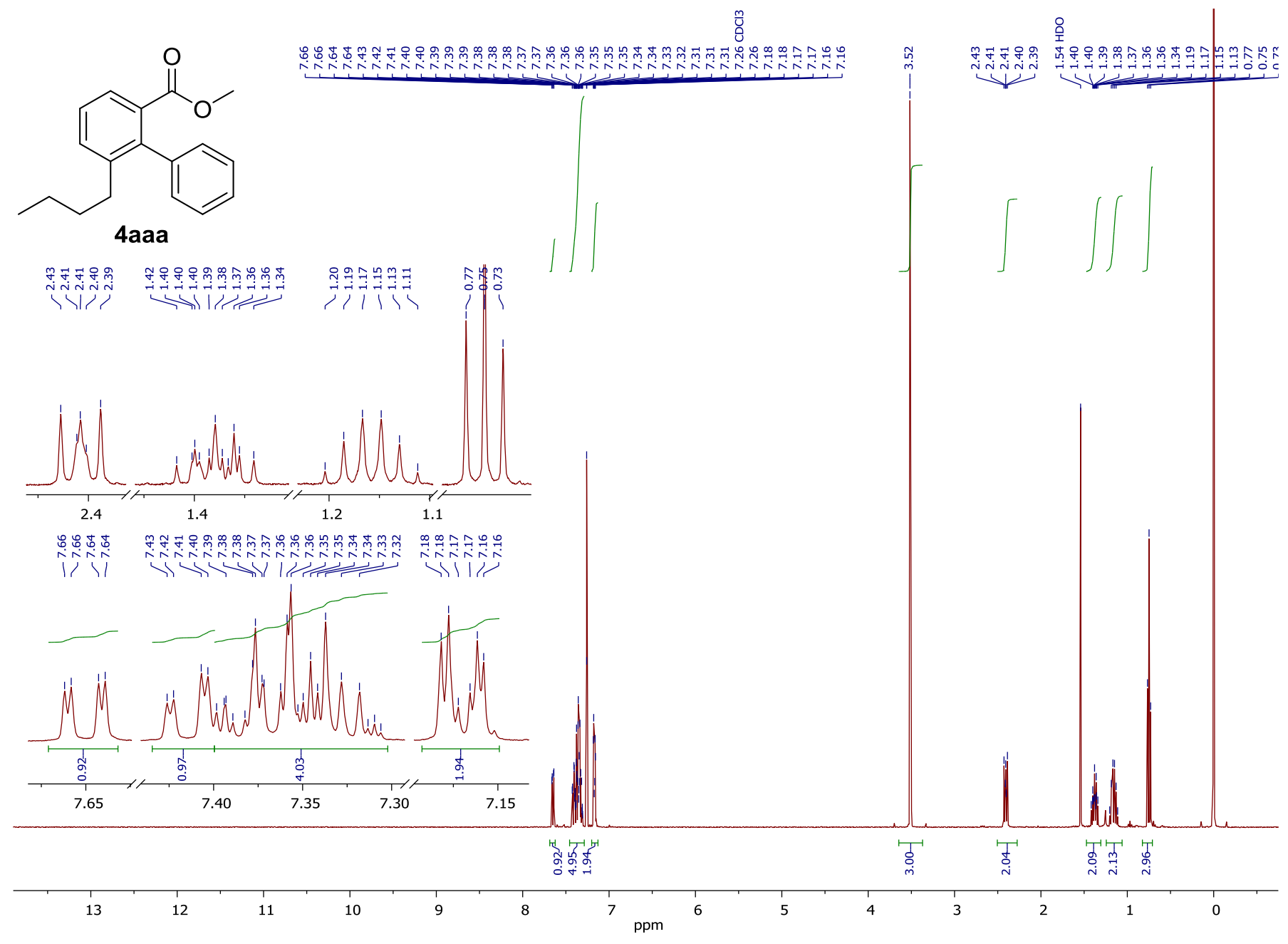


<smiles>CCCCc1cccc(C(=O)OC)c1-c1ccccc1</smiles><smiles></smiles>

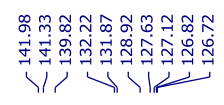

泀

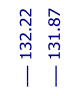

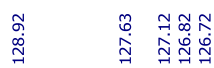

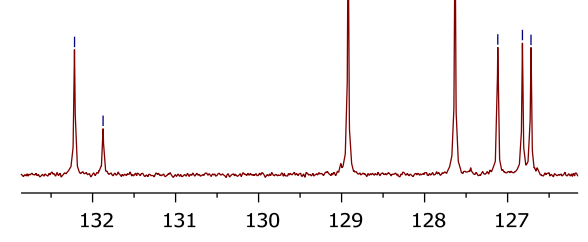

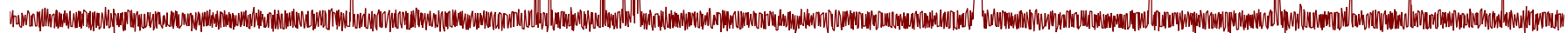

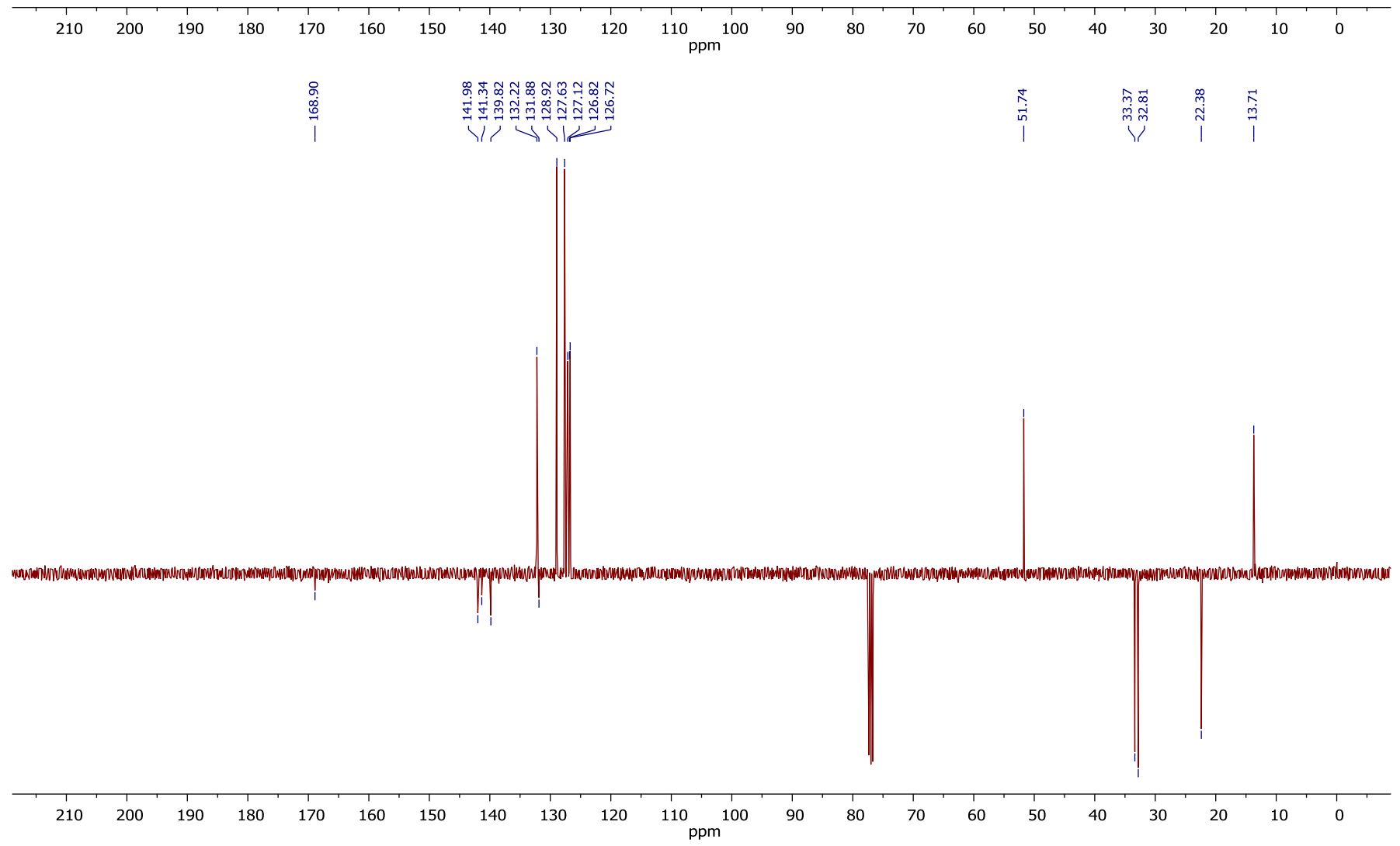


<smiles>CCCCc1cc(Cl)cc(C(=O)OC)c1-c1ccccc1</smiles>

范

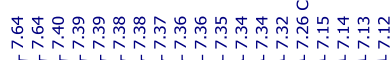

일

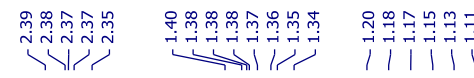

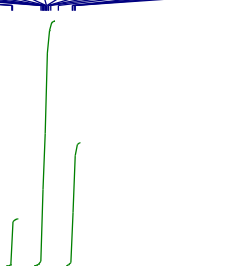

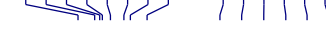

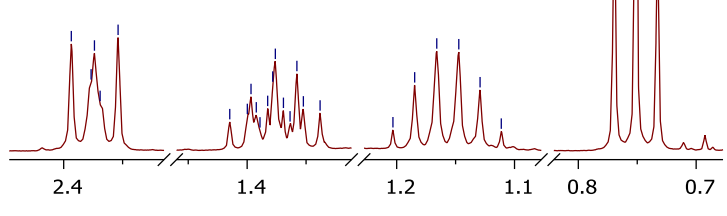

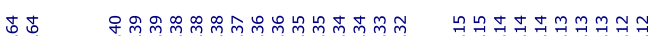

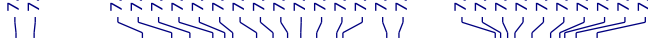
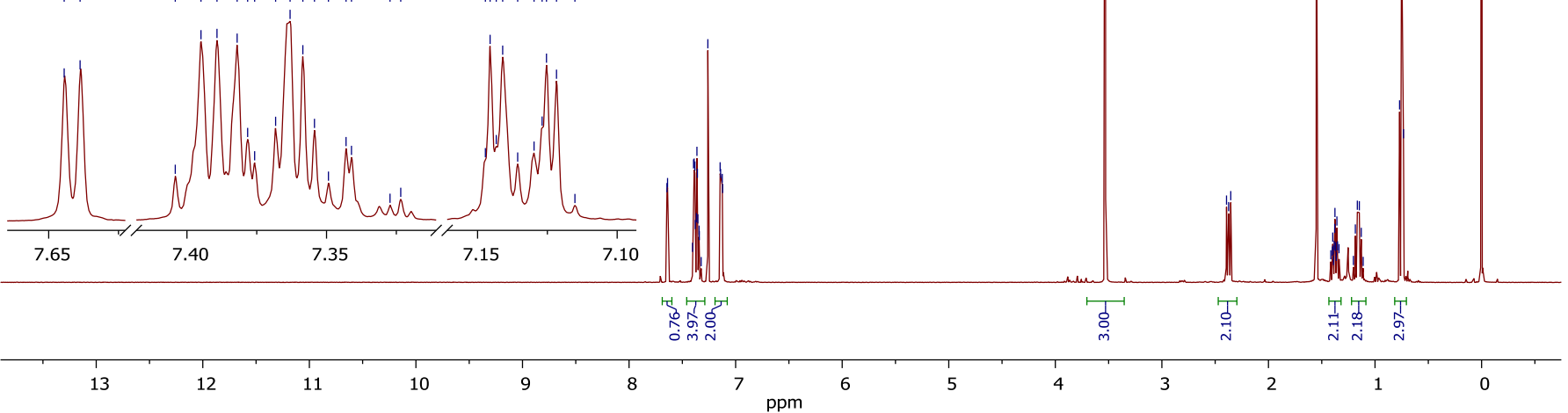

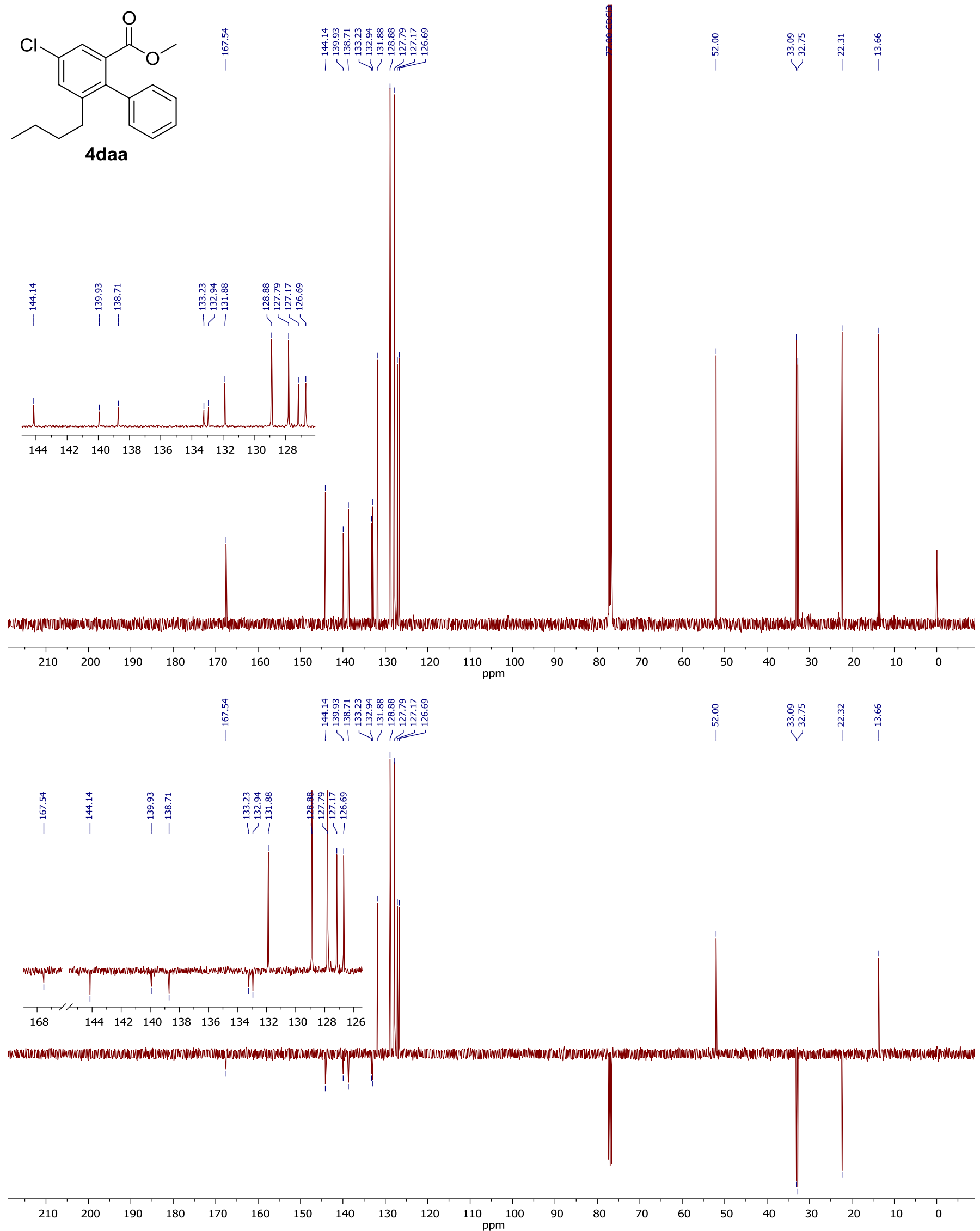

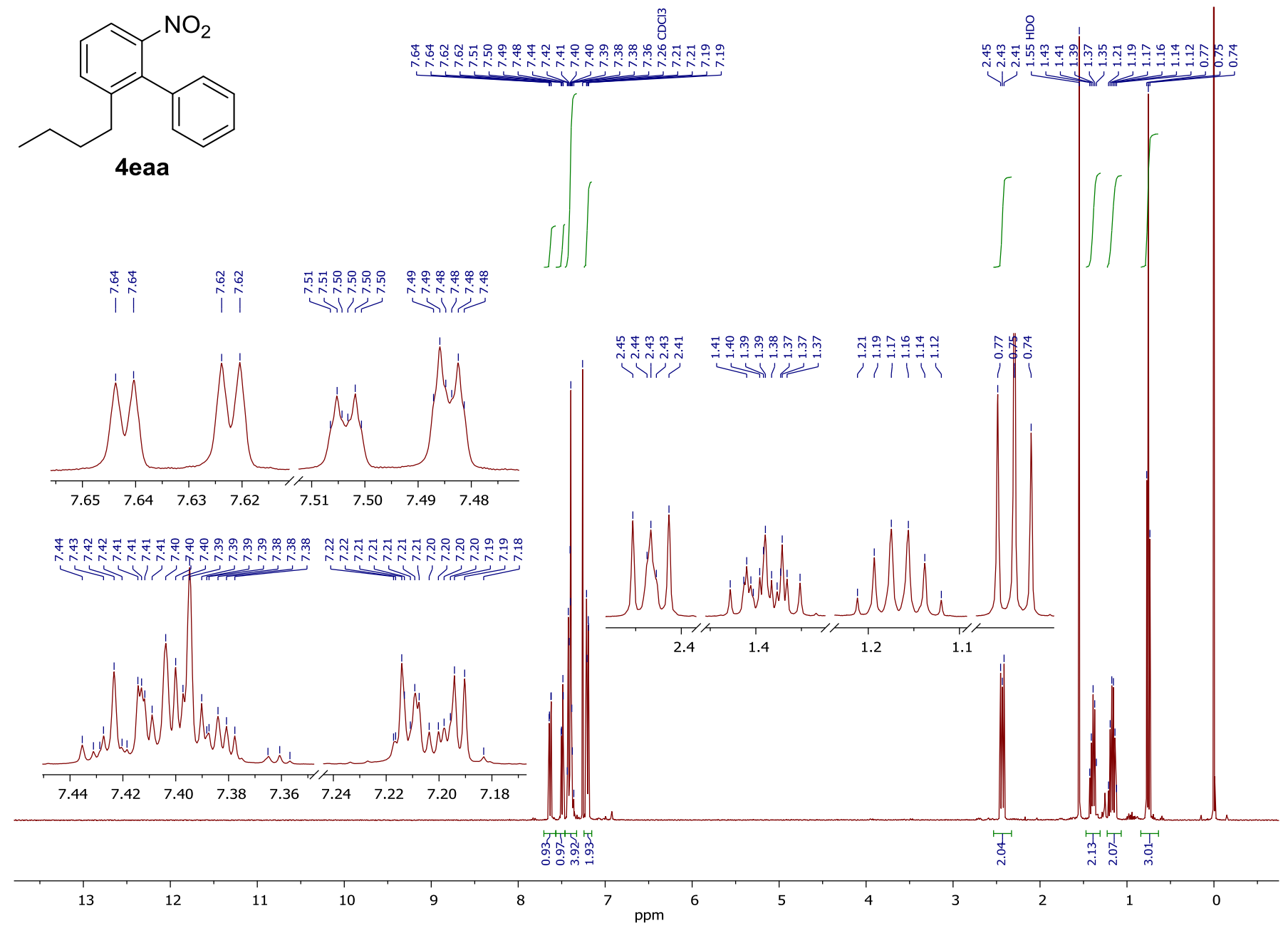
<smiles>CCCCc1cccc([N+](=O)[O-])c1-c1ccccc1</smiles>
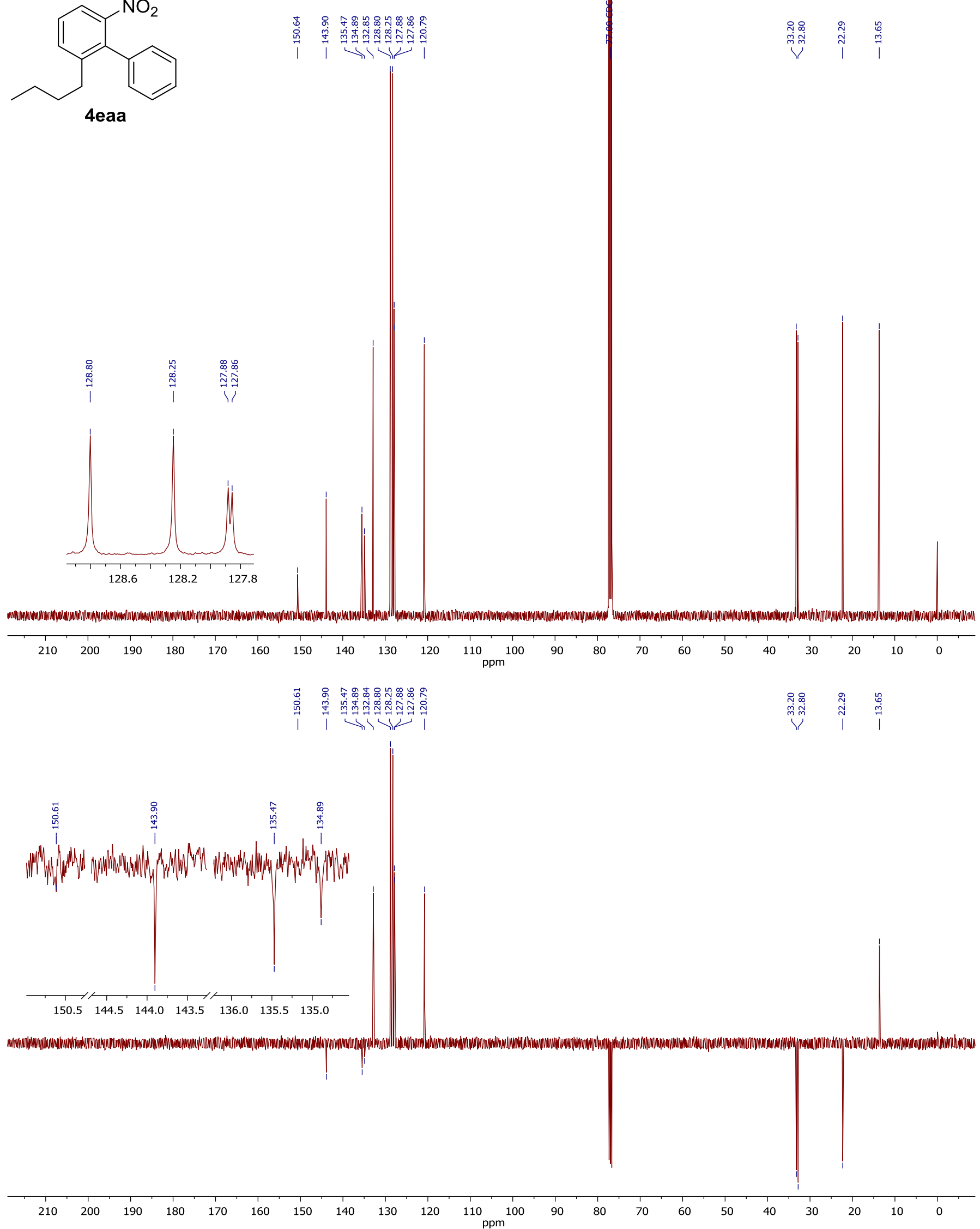


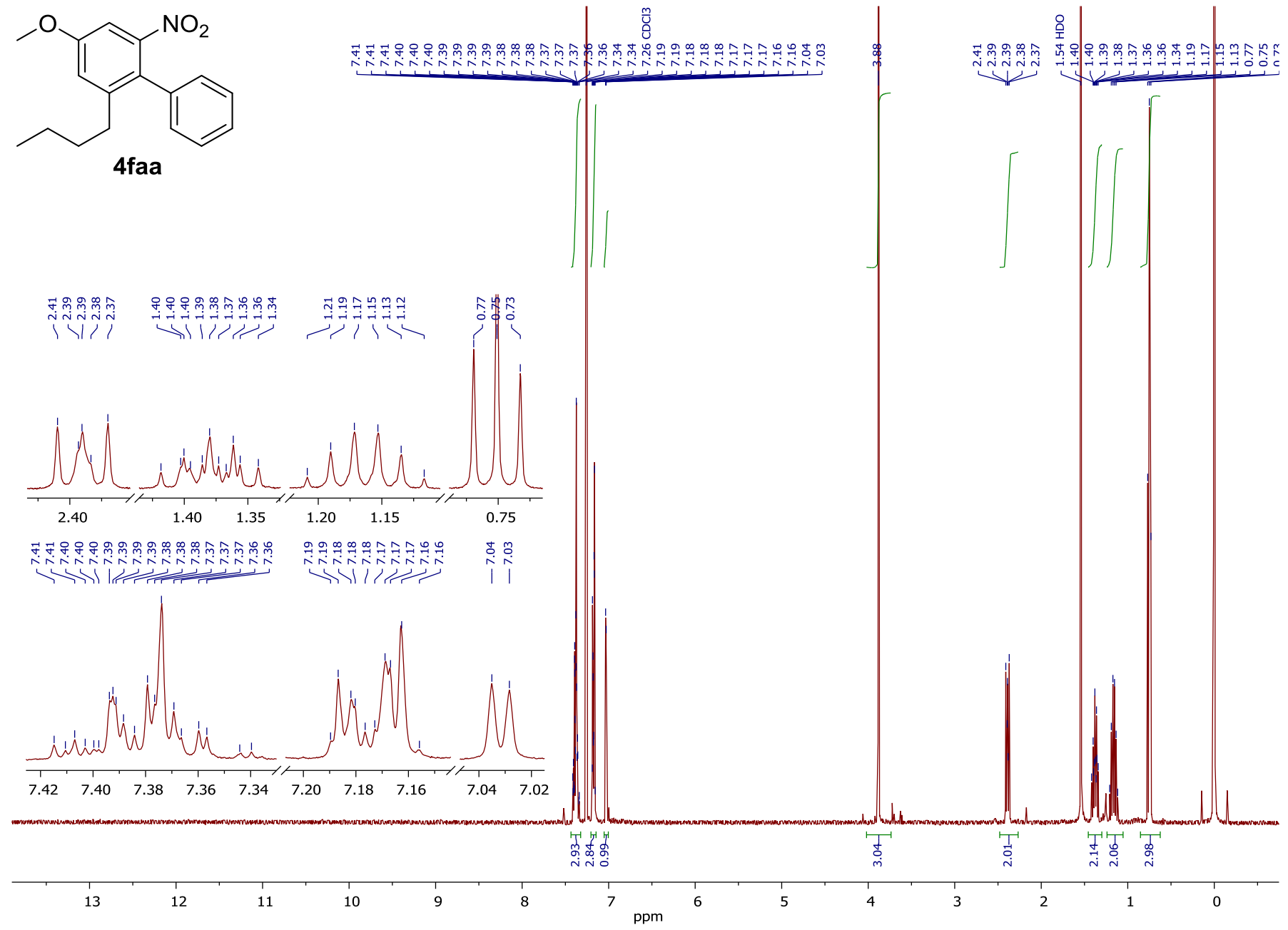


<smiles>CCCCc1cc(OC)cc([N+](=O)[O-])c1-c1ccccc1</smiles>

$4 f a a$
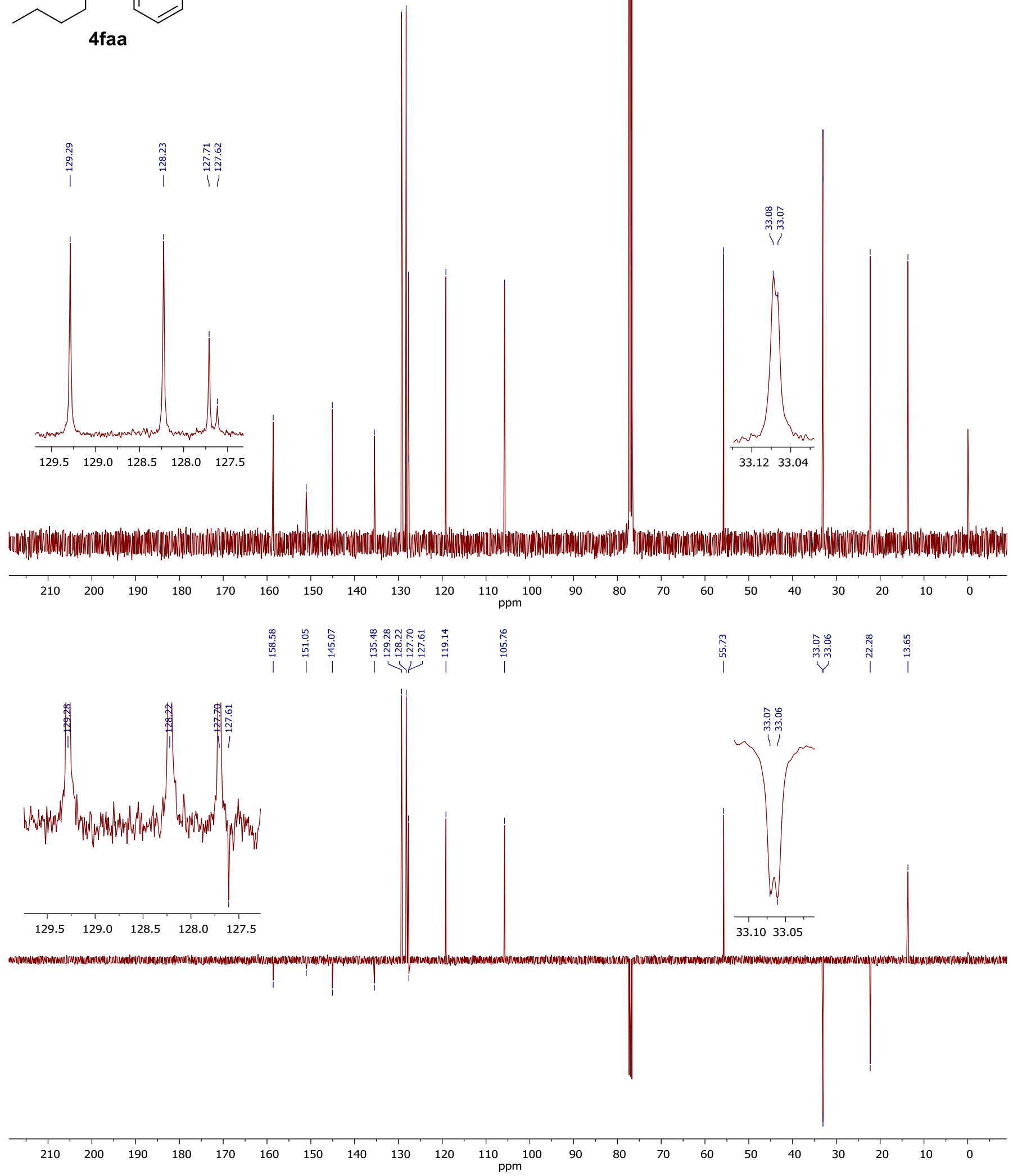
<smiles>CCCCc1cccc(C(F)(F)F)c1-c1ccccc1</smiles>

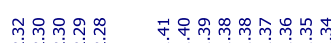

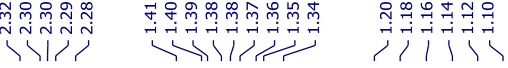

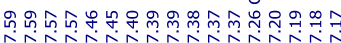

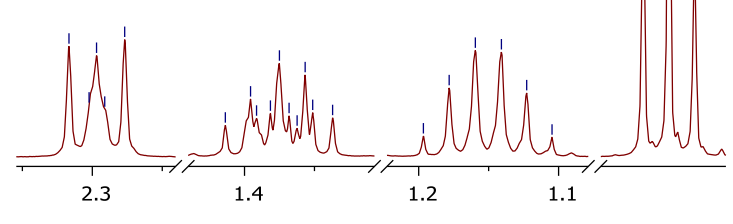

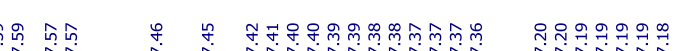

il
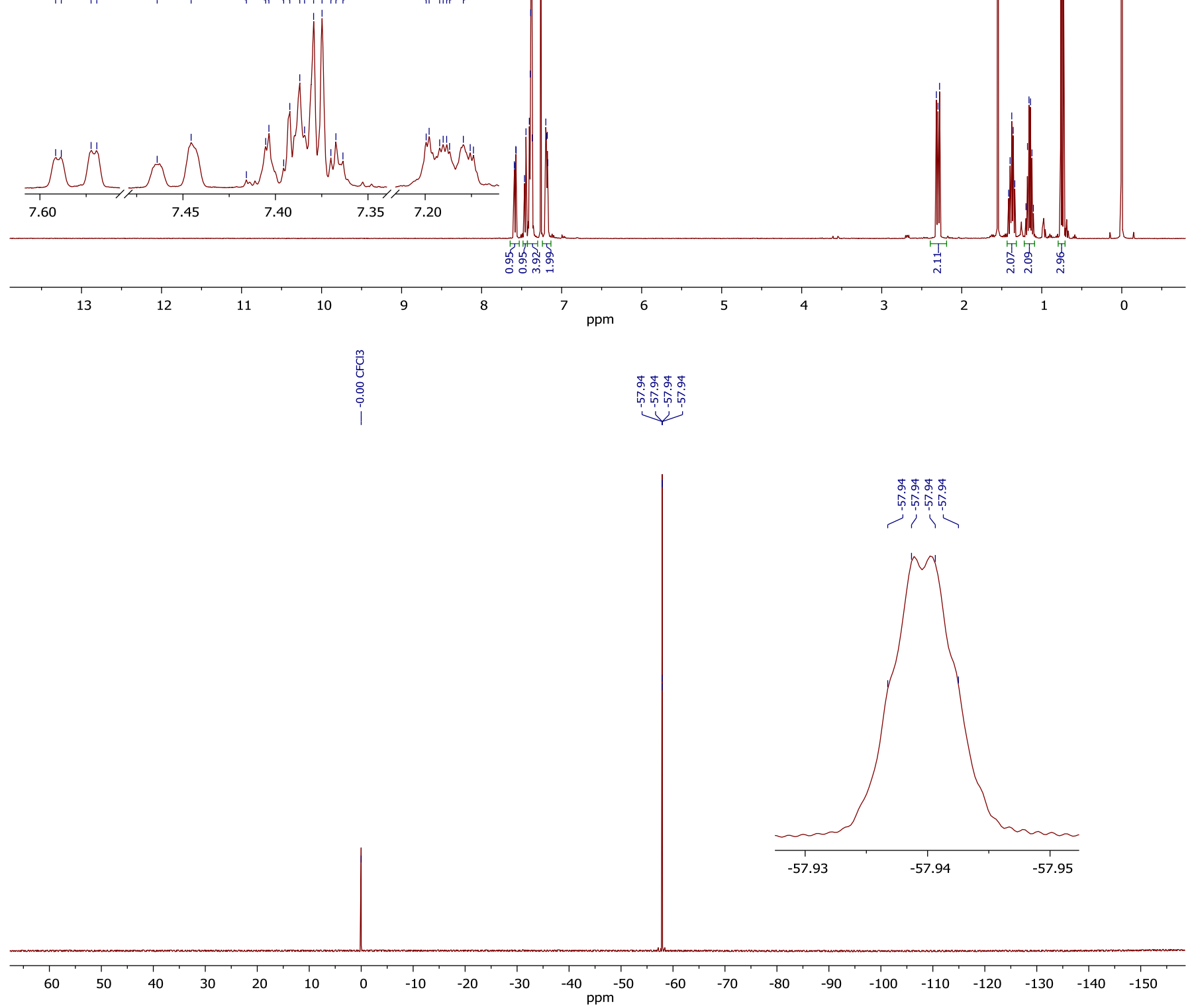


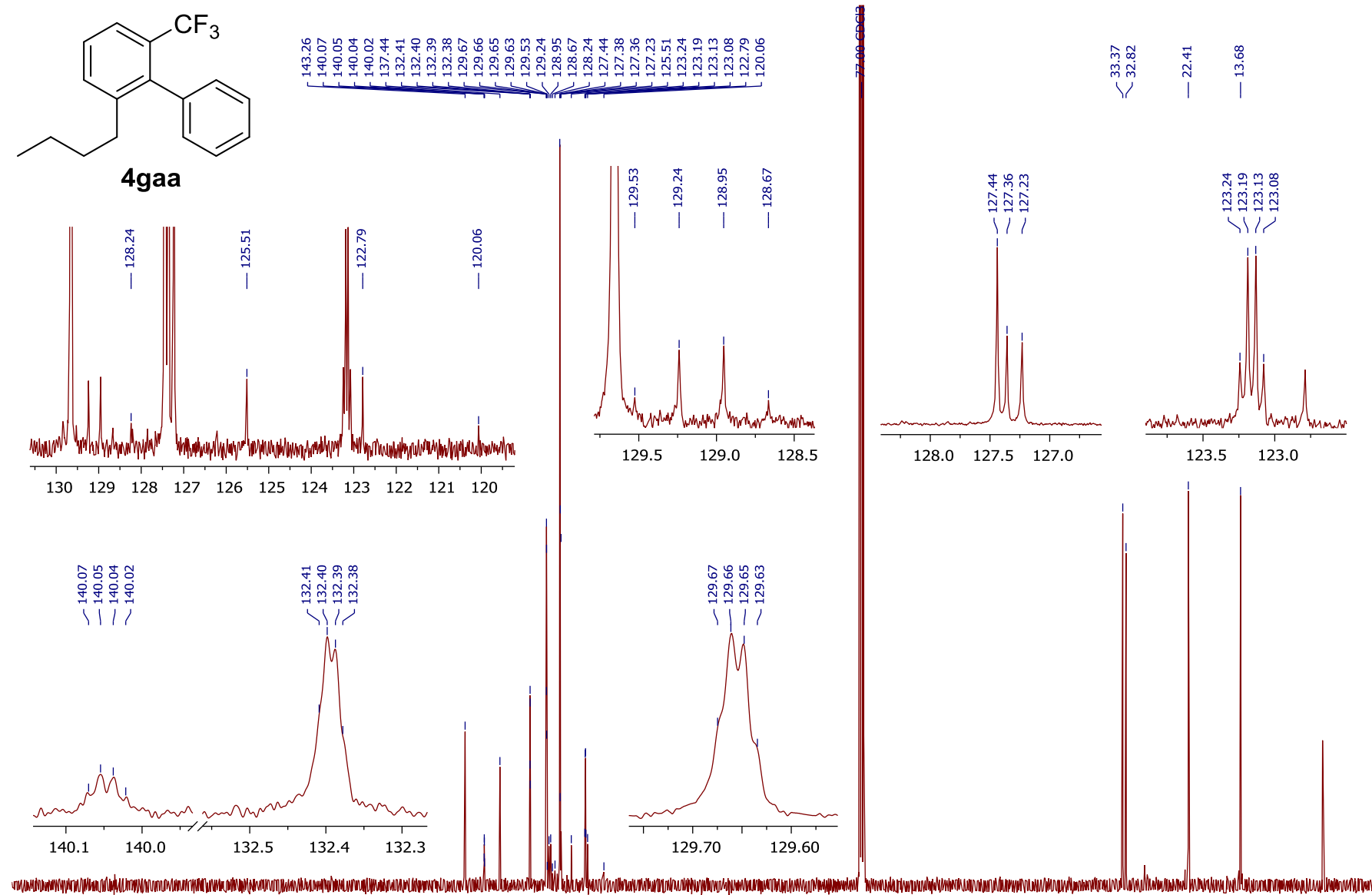

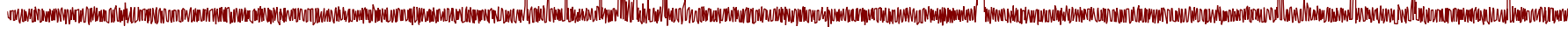

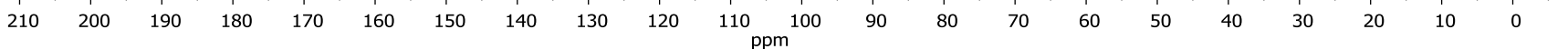

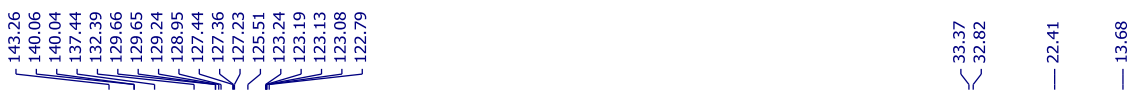

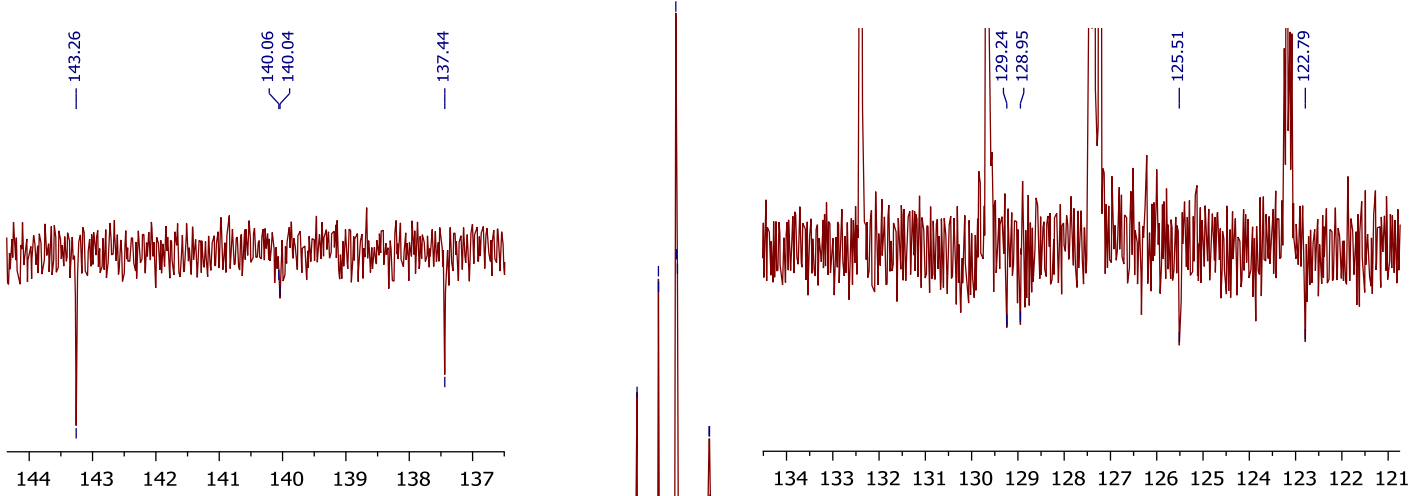

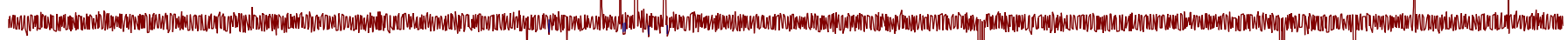

$\begin{array}{lllllllllllllllllllllll}210 & 200 & 190 & 180 & 170 & 160 & 150 & 140 & 130 & 120 & 110 & 100 & 90 & 80 & 70 & 60 & 50 & 40 & 30 & 20 & 10 & 0\end{array}$


<smiles>CCCCc1c(F)ccc(C(F)(F)F)c1-c1ccccc1</smiles>

4haa

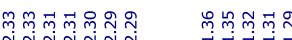

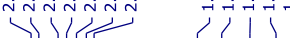

8

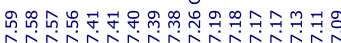
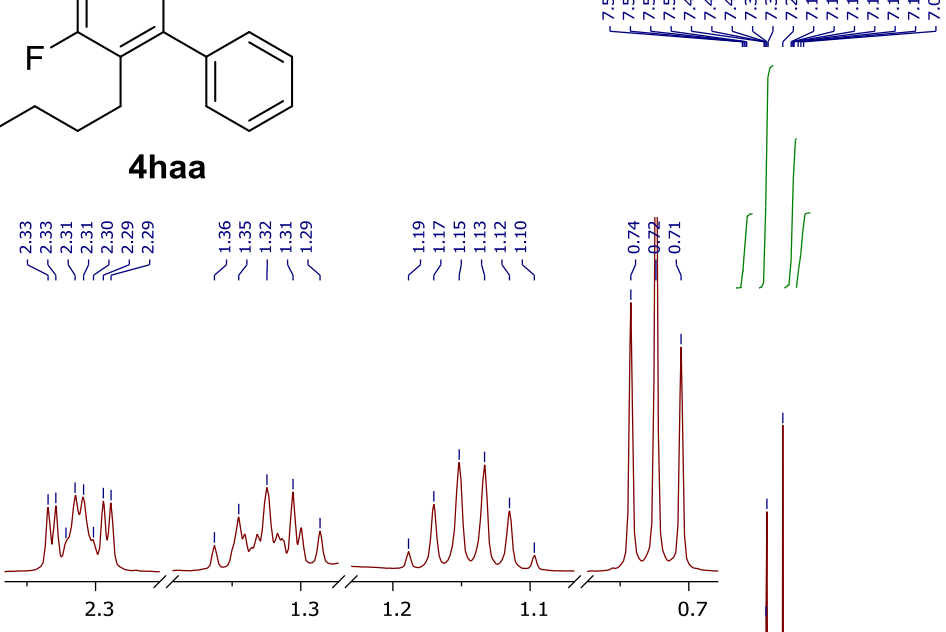

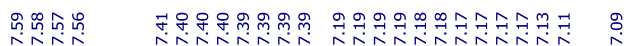

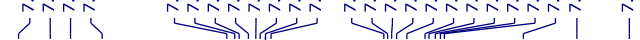
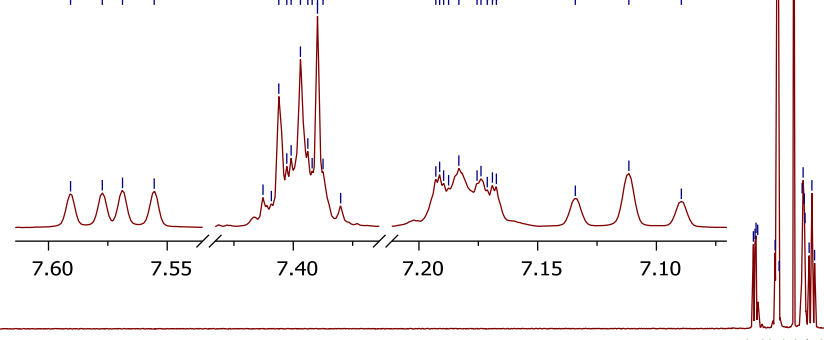

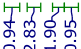

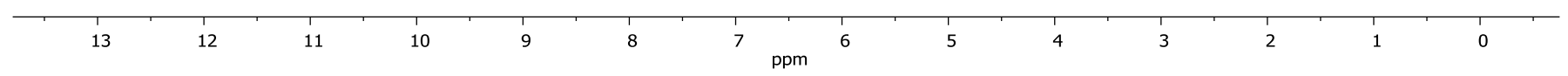

0

फिकिजिएकी

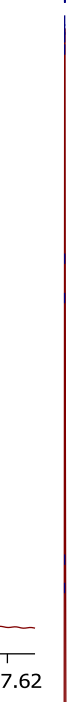

ț

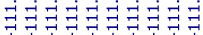

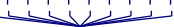

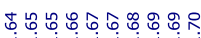
$\exists \exists \exists \exists \exists \exists \exists \exists \exists$

111111111

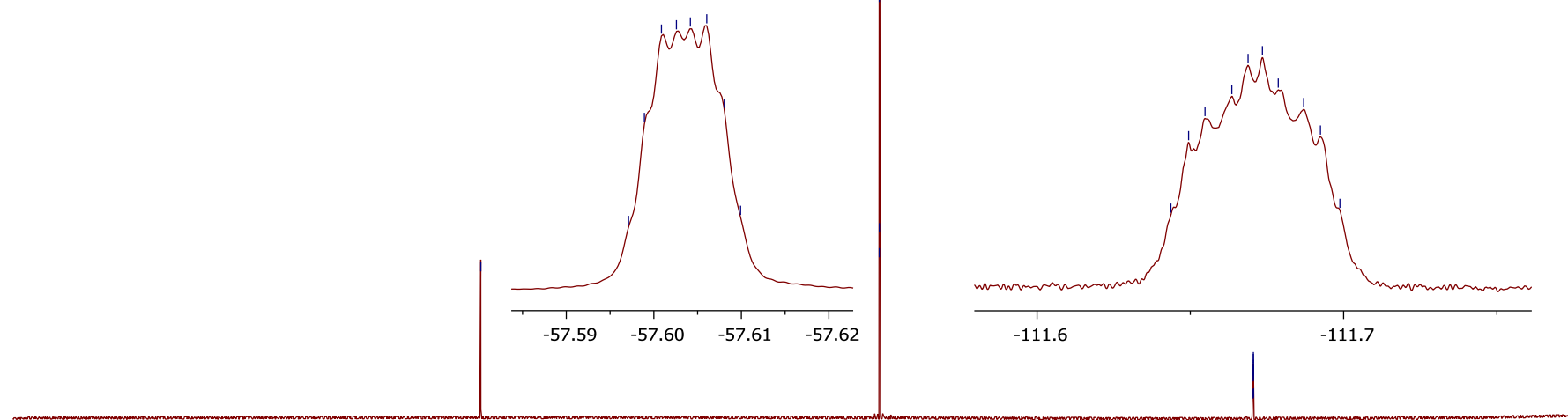


<smiles>CCCCc1c(F)ccc(C(F)(F)F)c1-c1ccccc1</smiles>

4haa

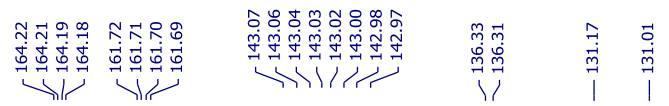

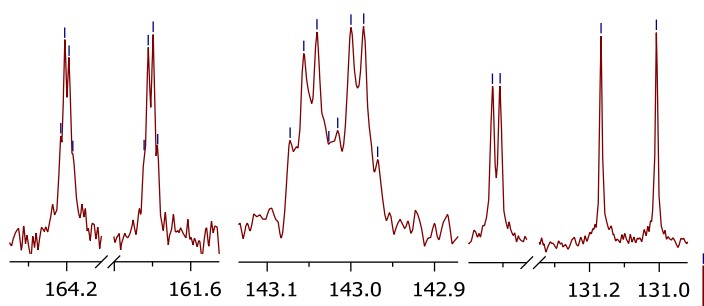

רึ||

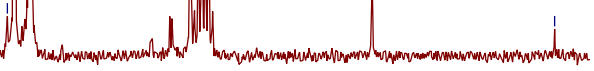
$\begin{array}{lllllllll}128 & 127 & 126 & 125 & 124 & 123 & 122 & 121 & 120\end{array}$ Yiv
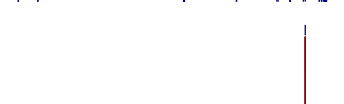

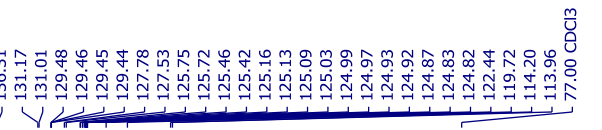

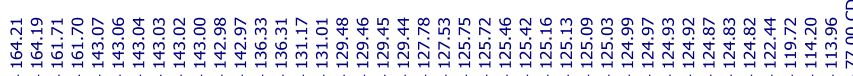

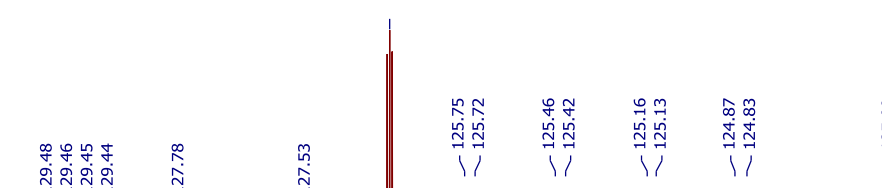

ตำ

2્木

रोiा?

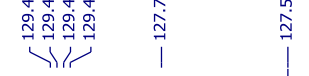
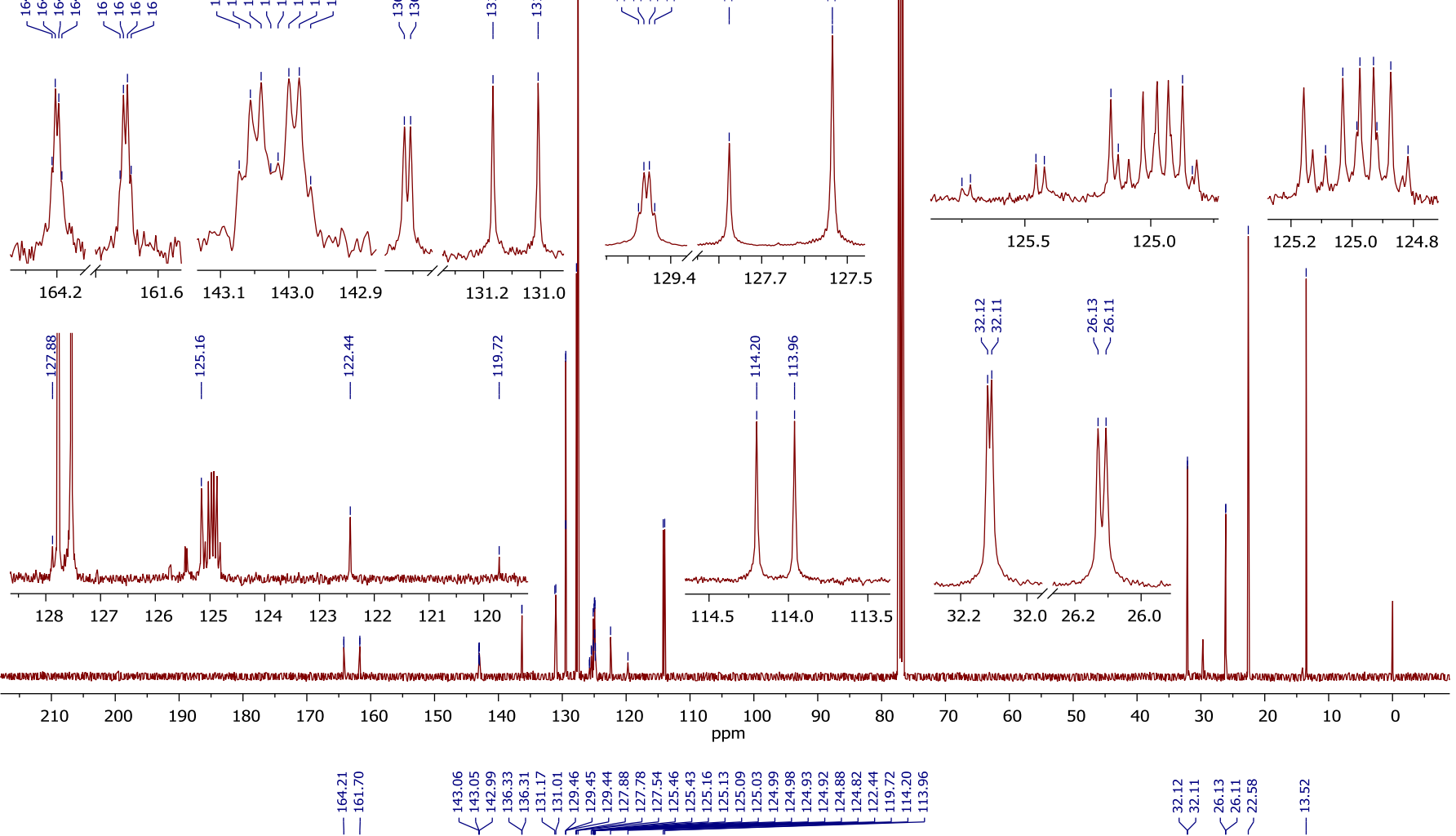

i

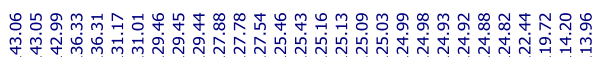

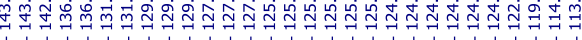

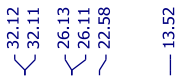

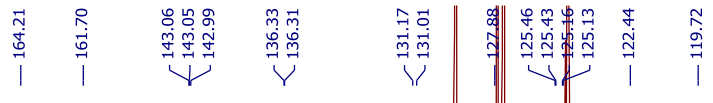
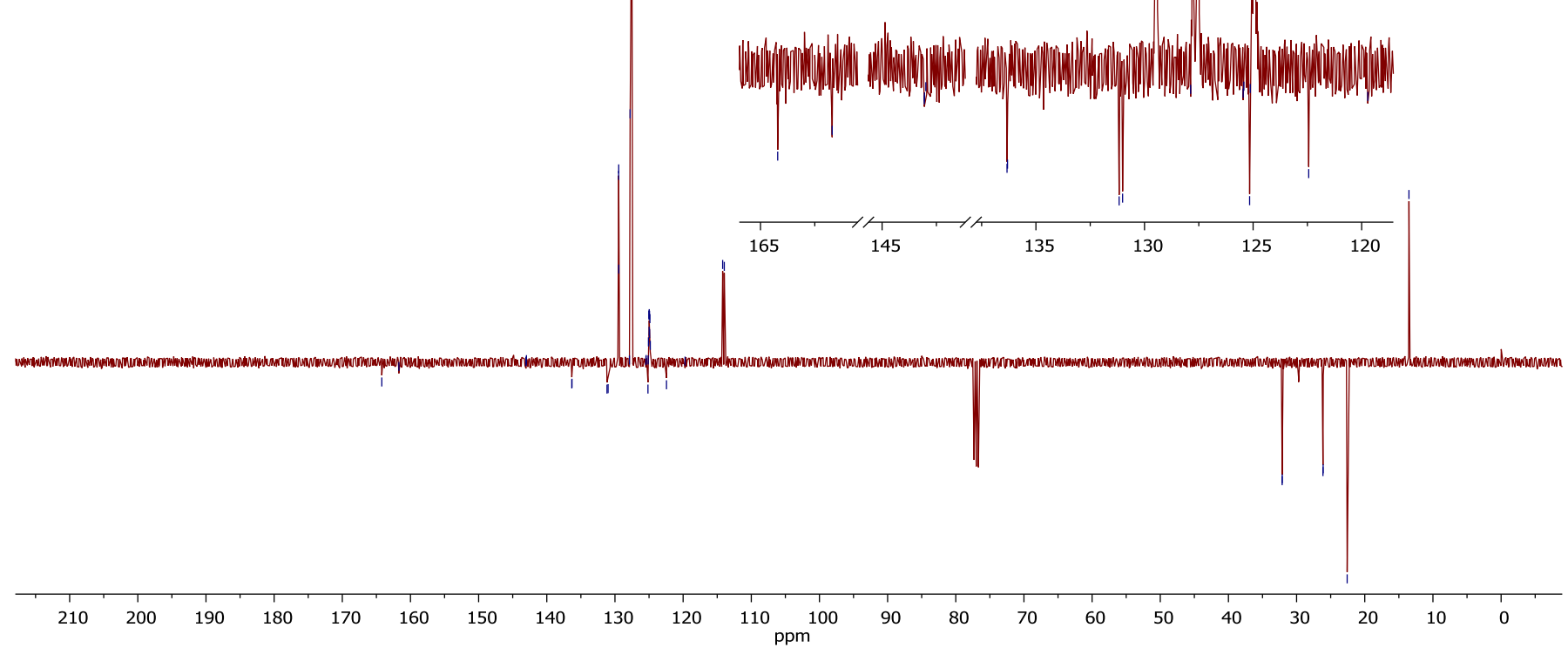

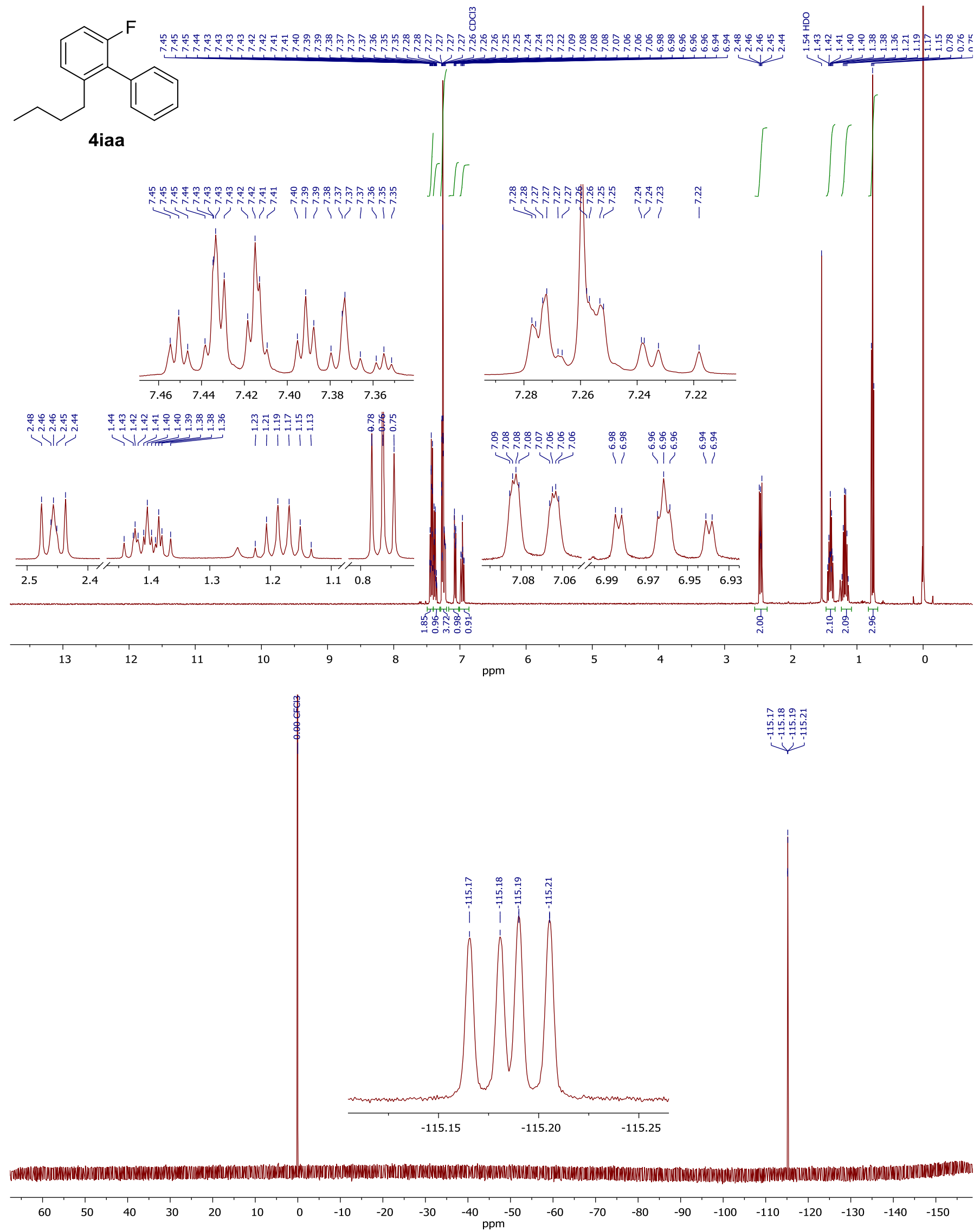

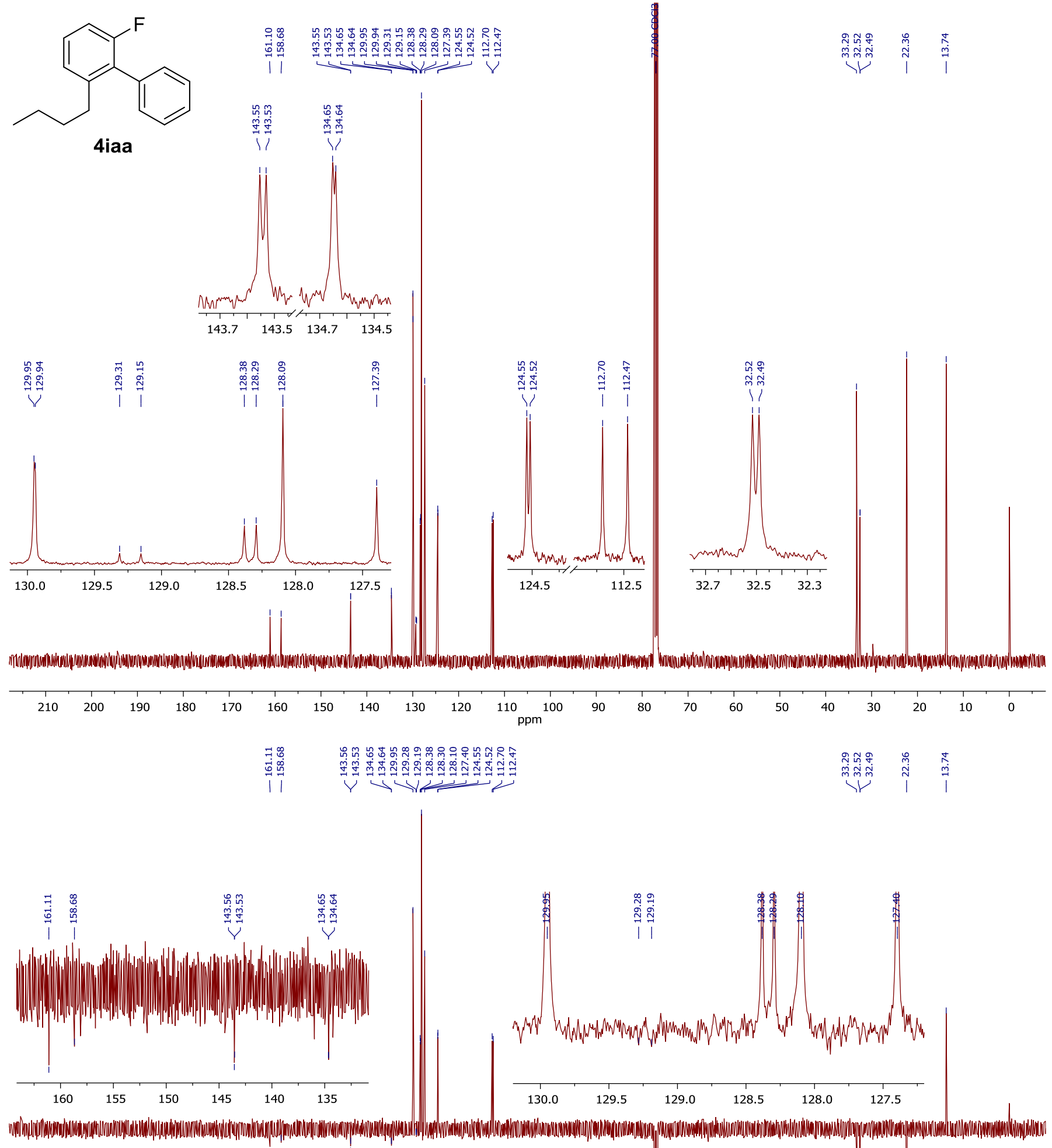

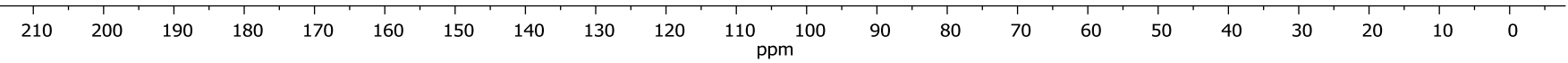




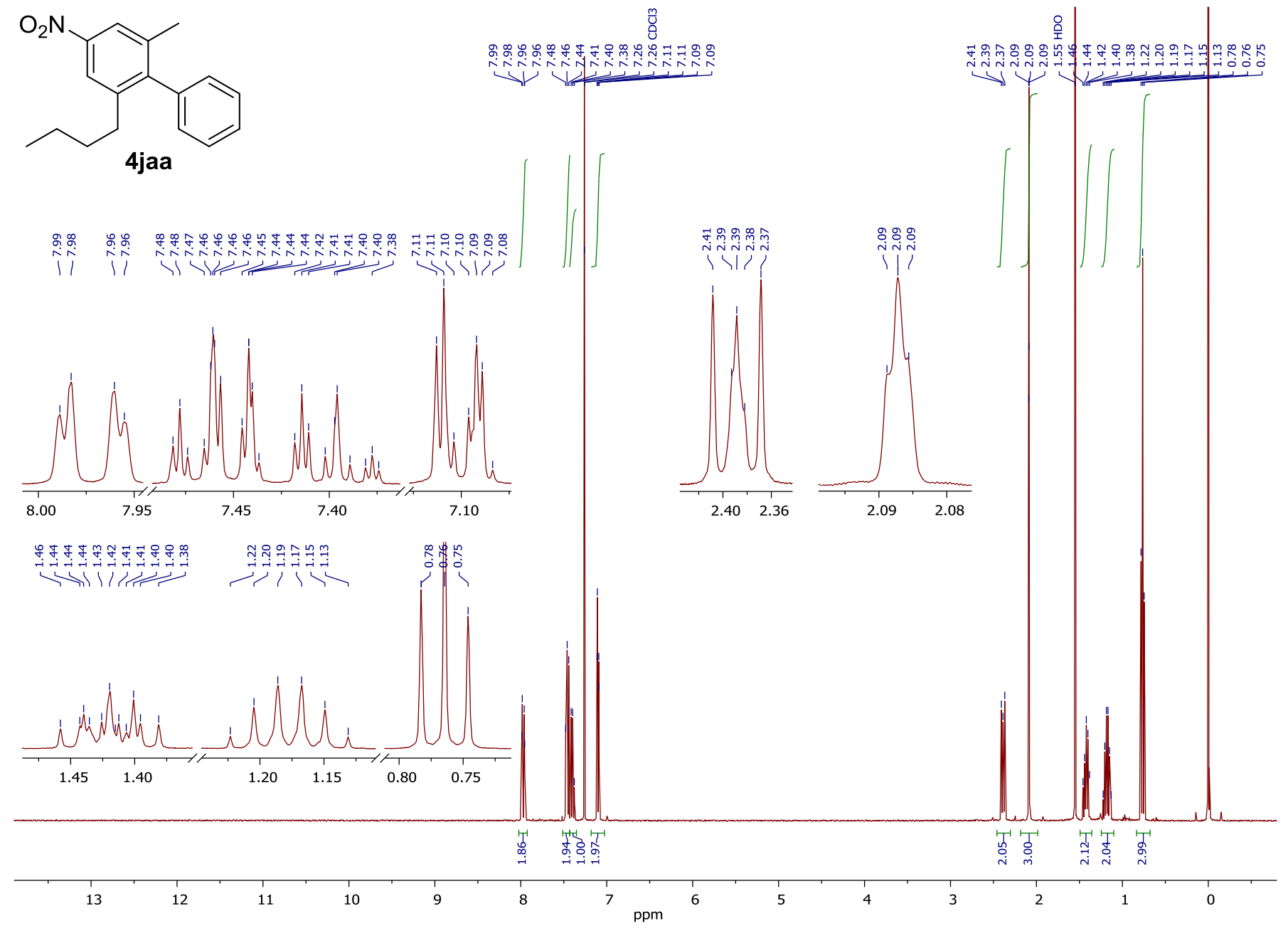



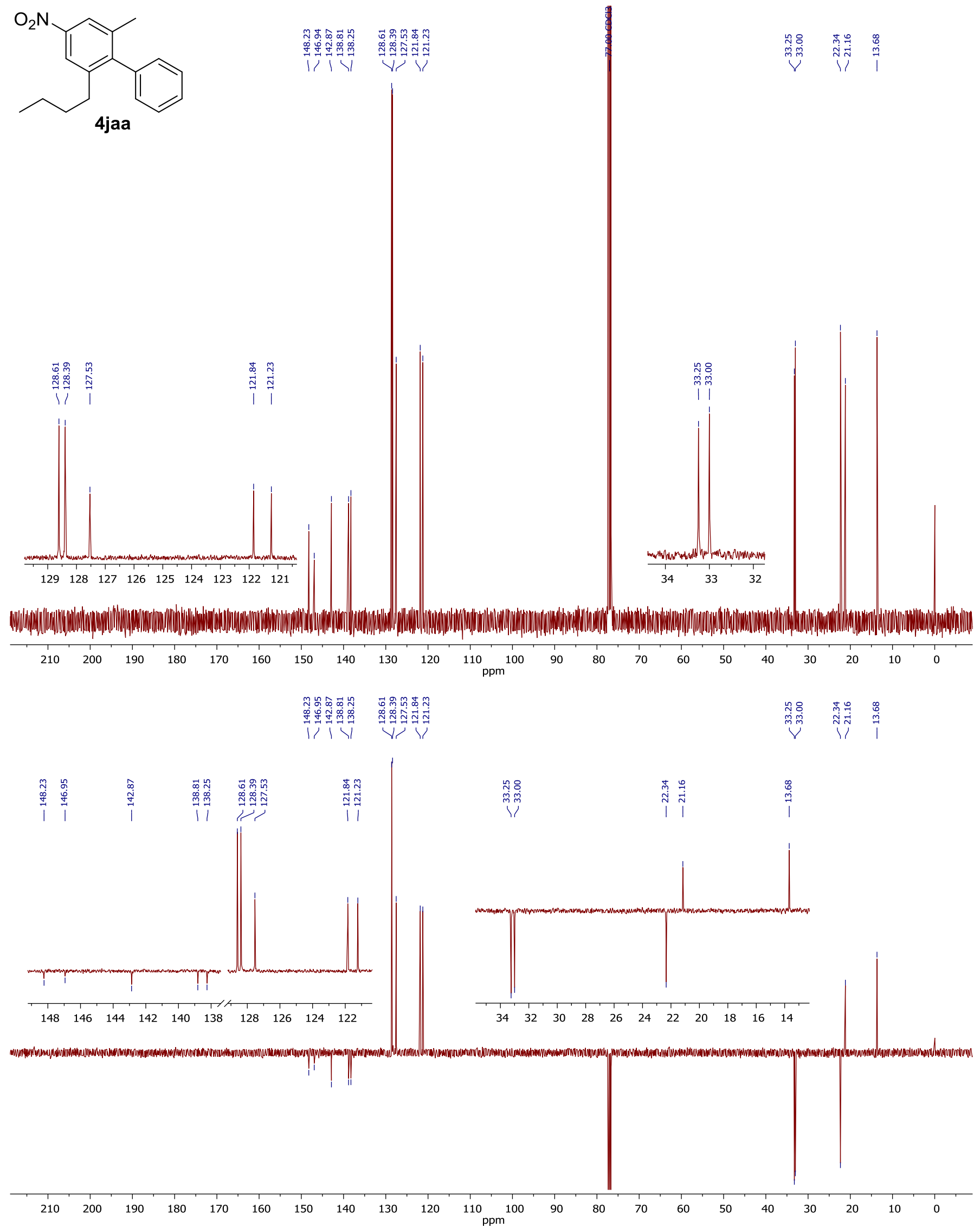


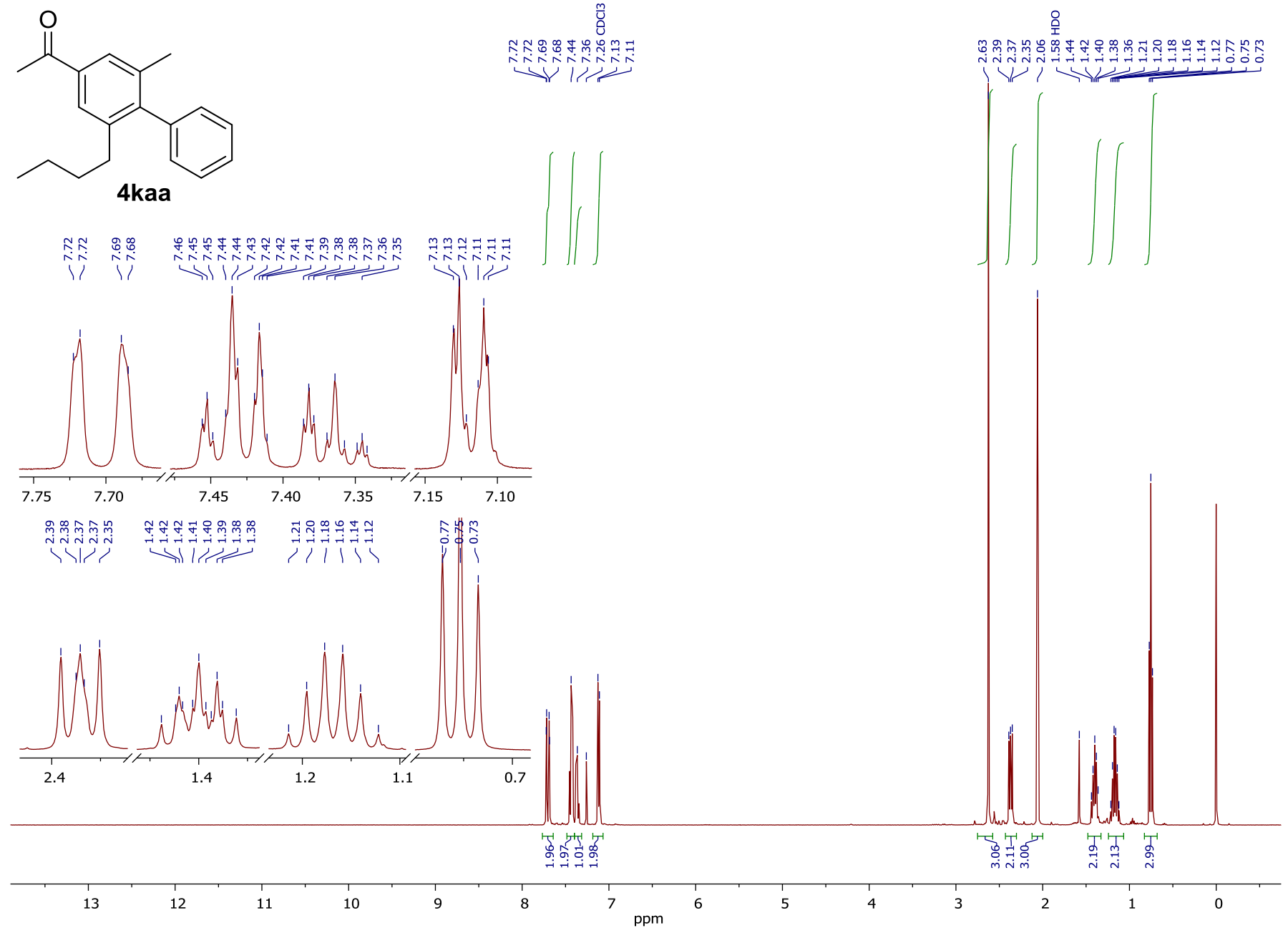



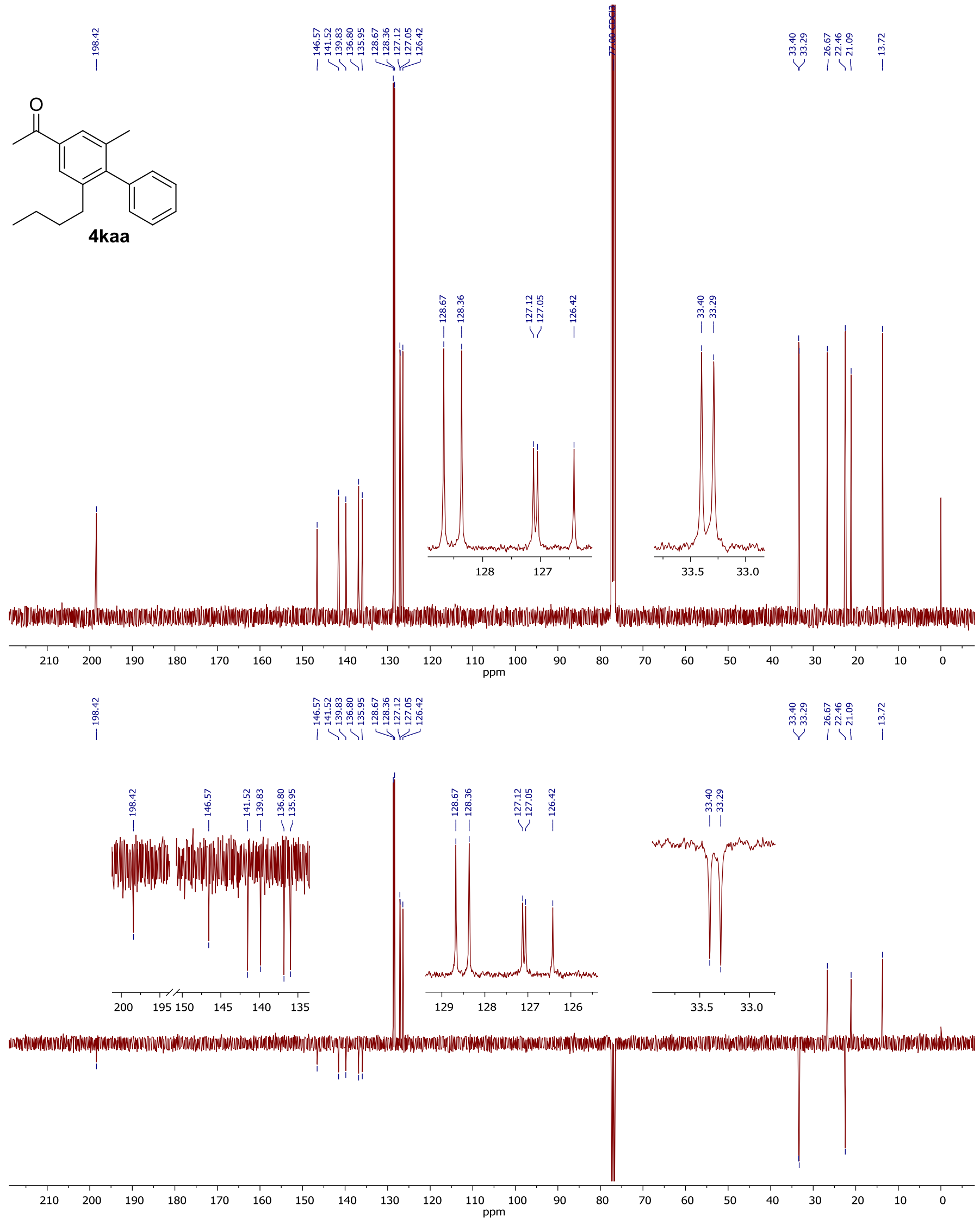
<smiles>CCCCc1cc(C=O)cc(OC)c1-c1ccccc1</smiles>
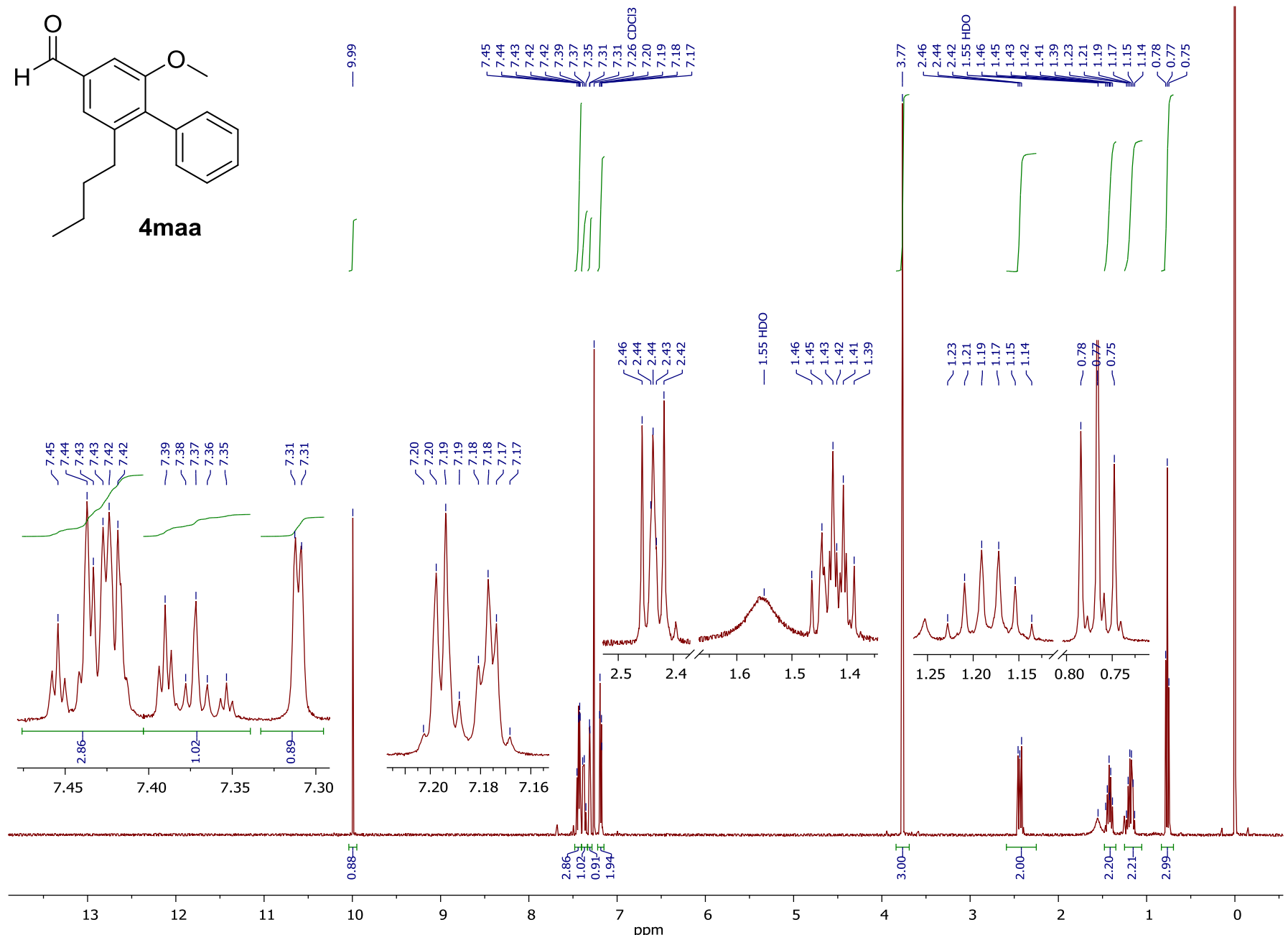


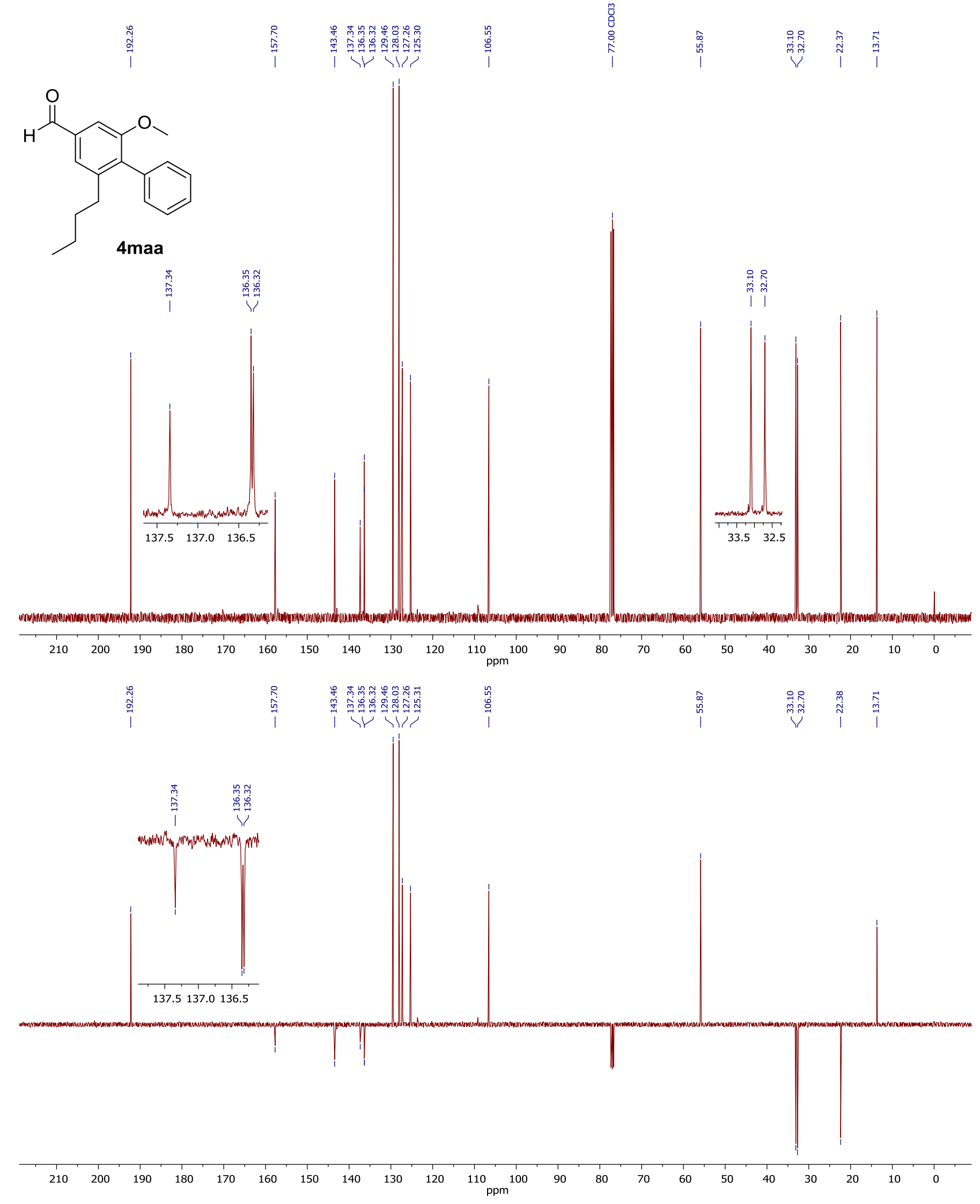



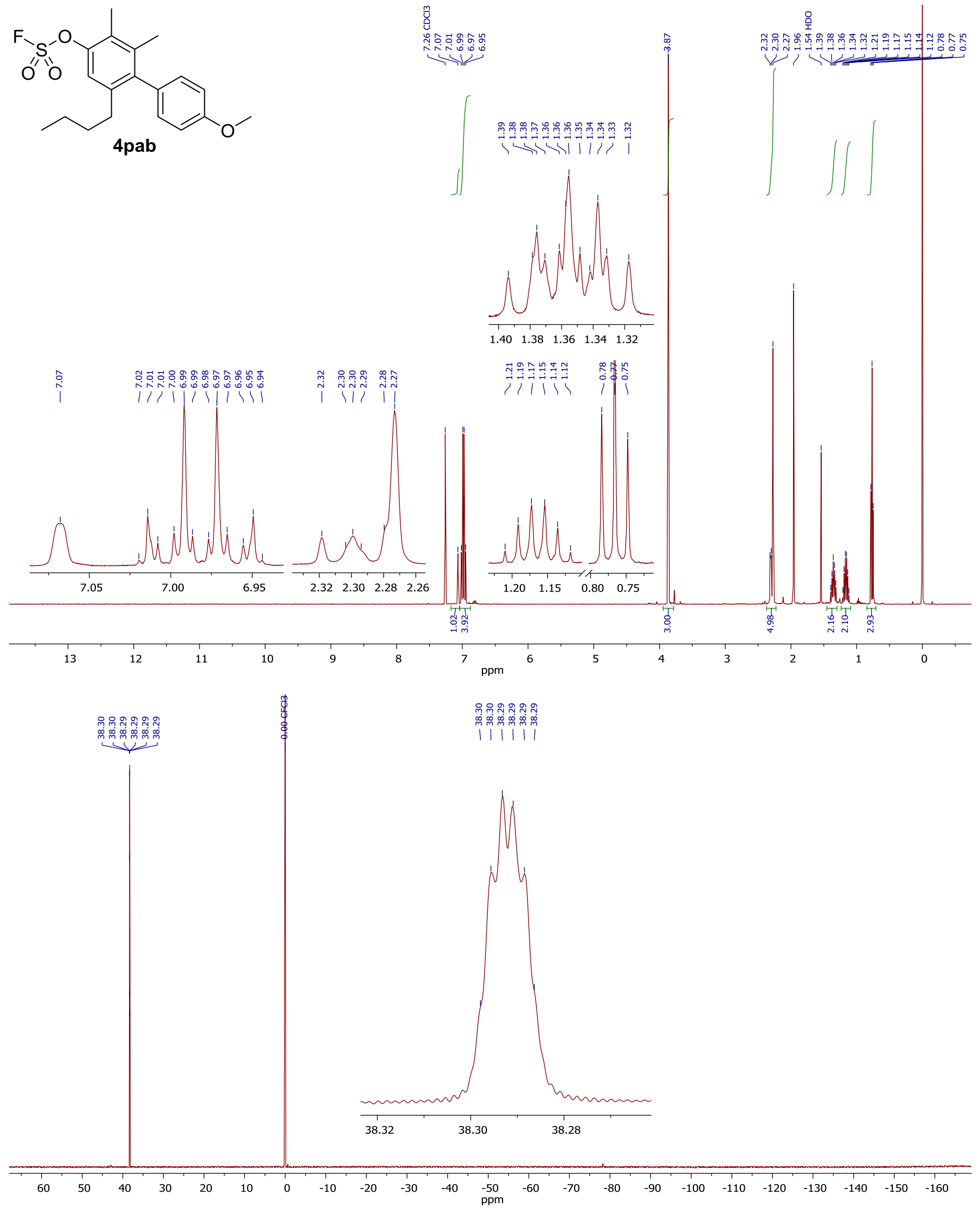


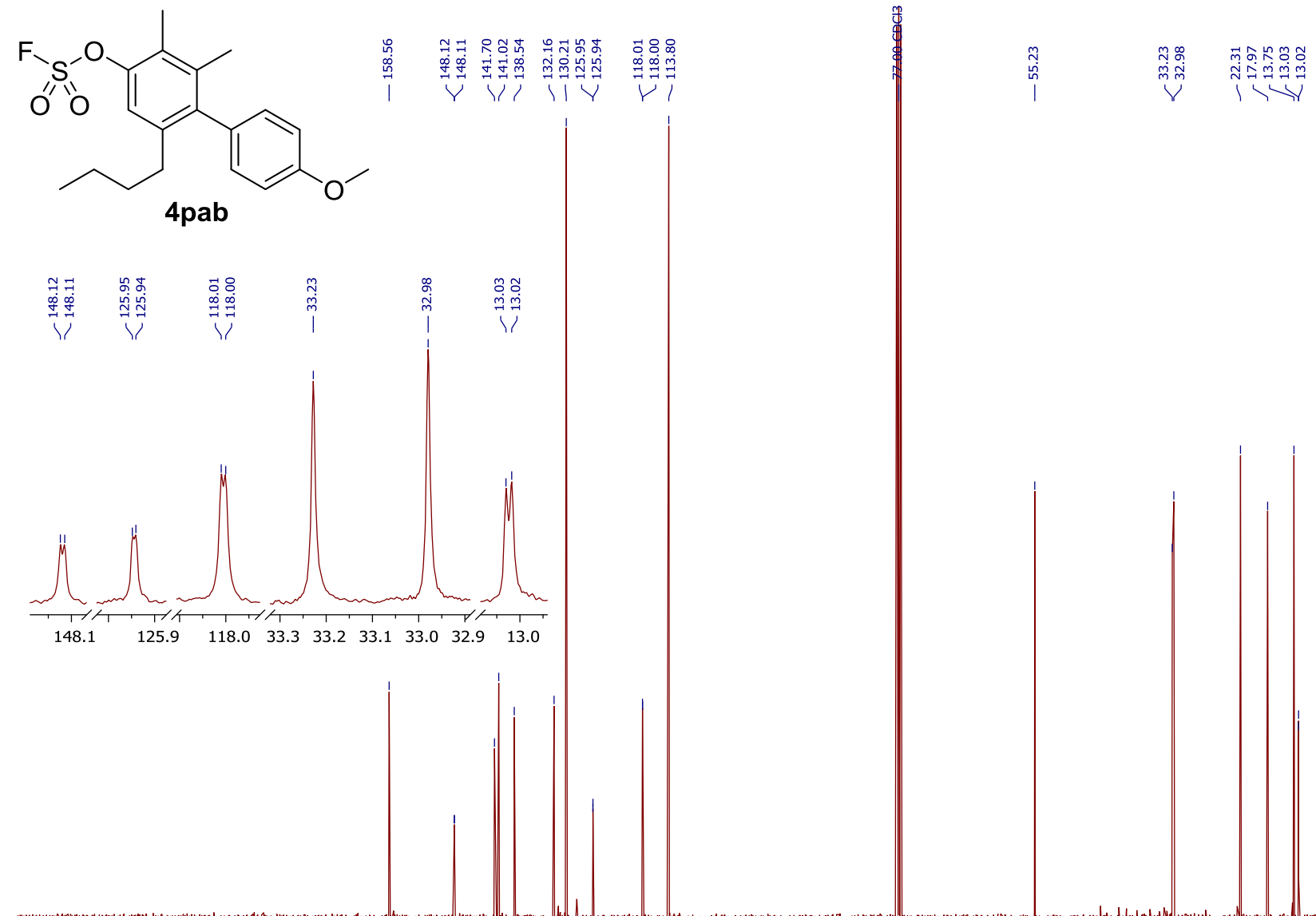

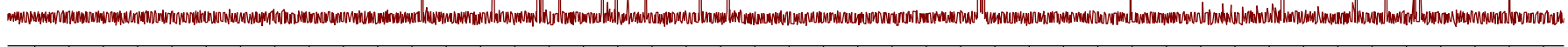
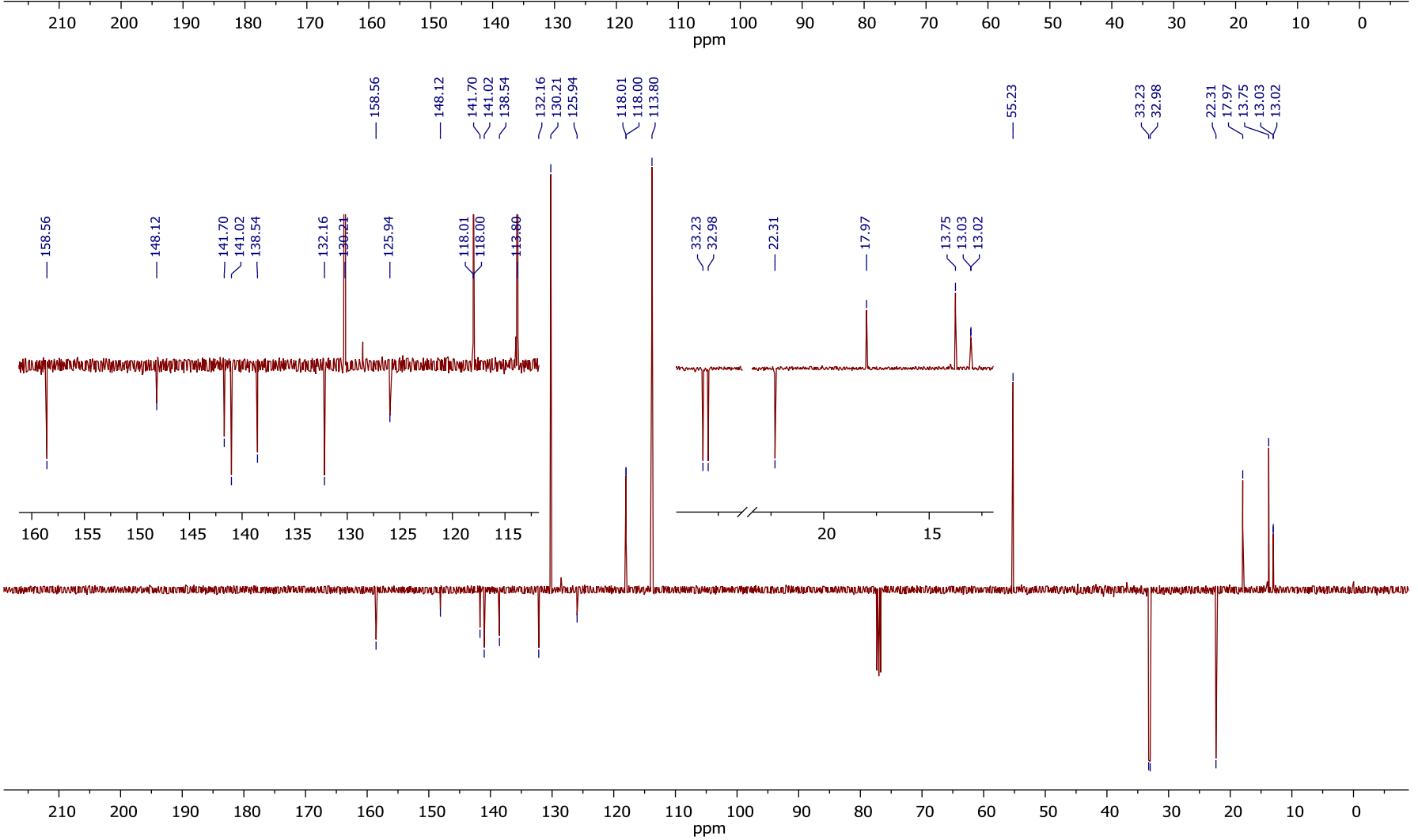


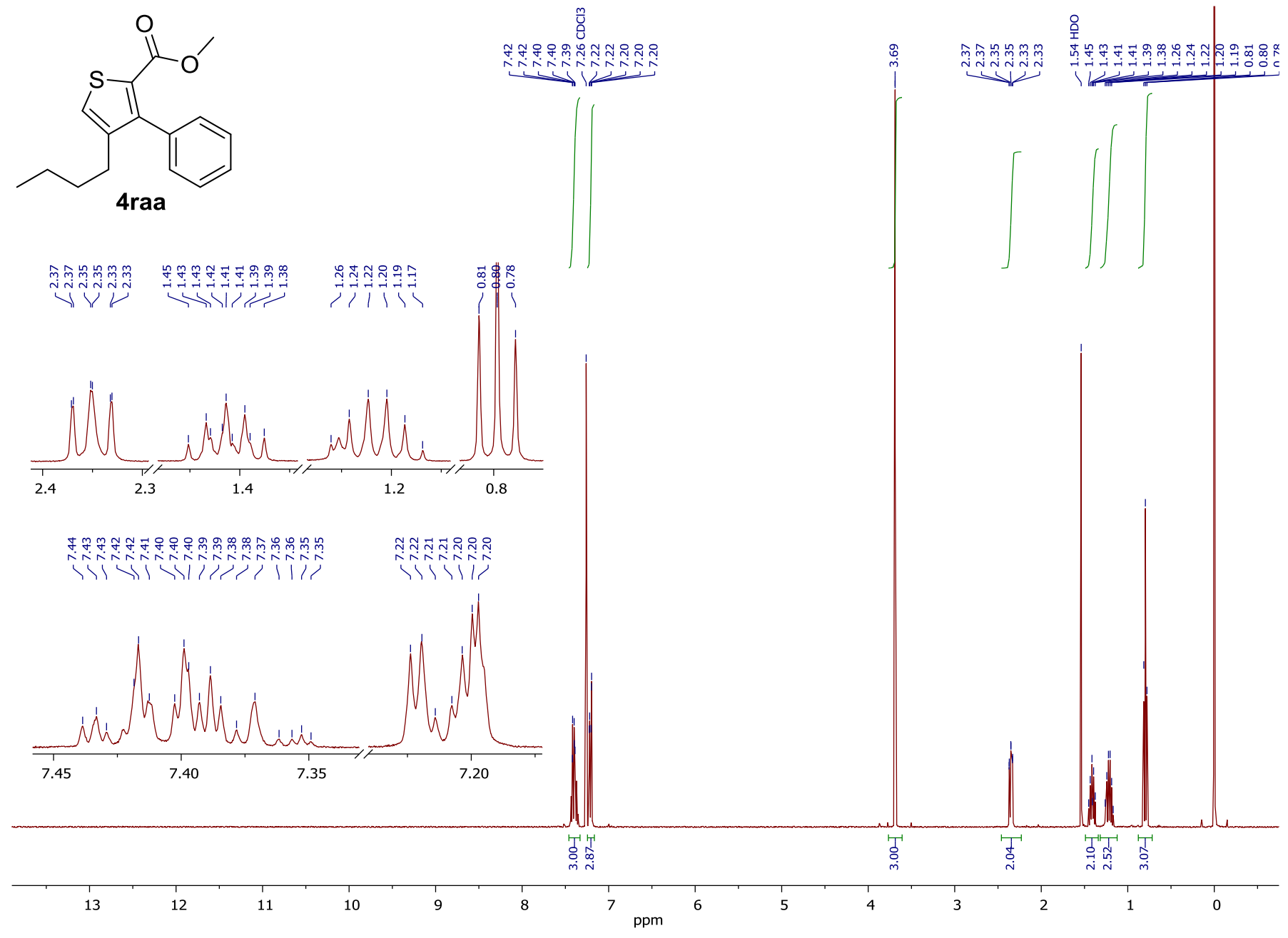



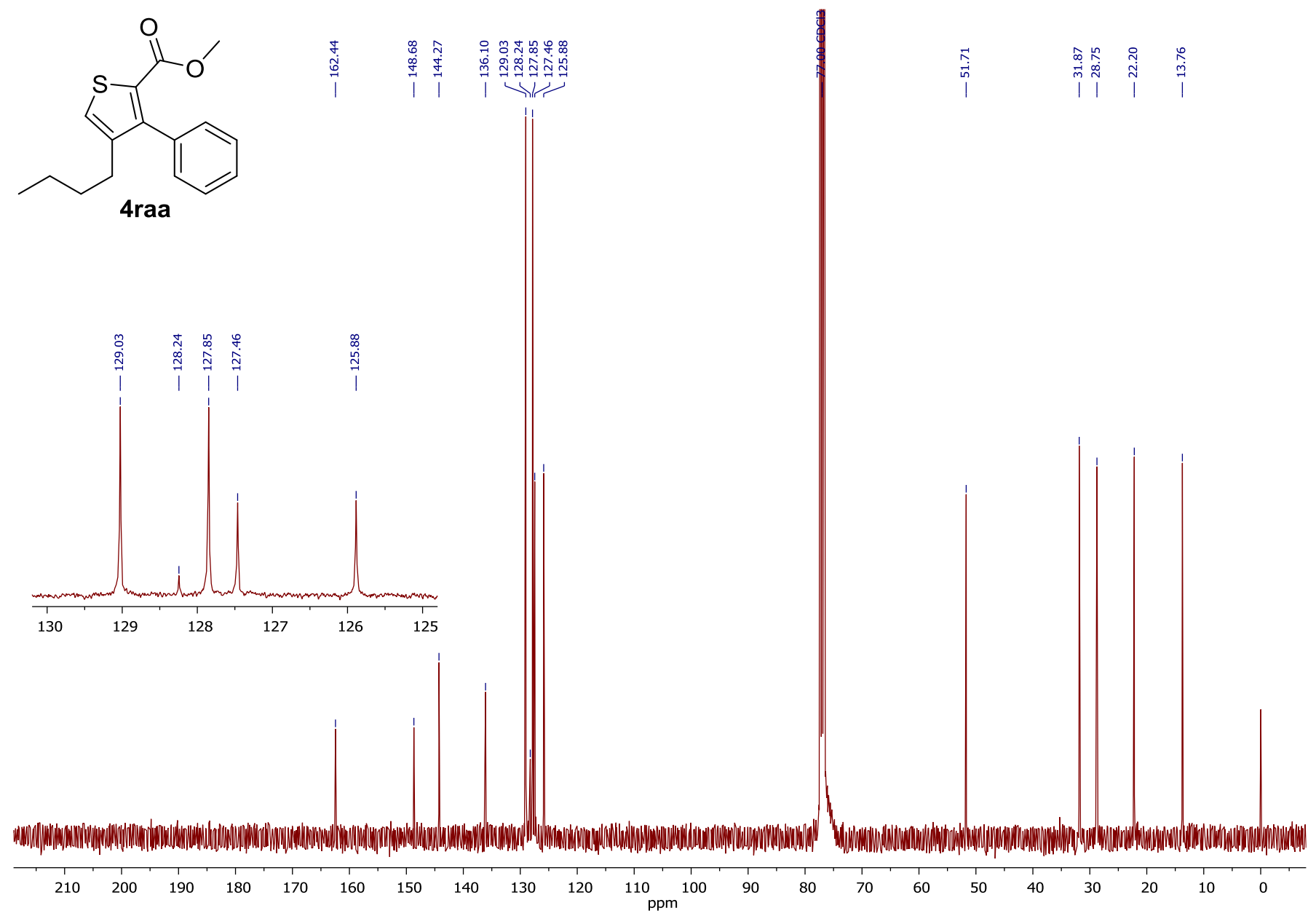

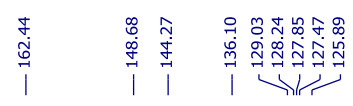

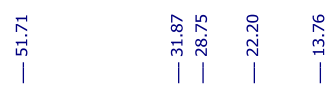
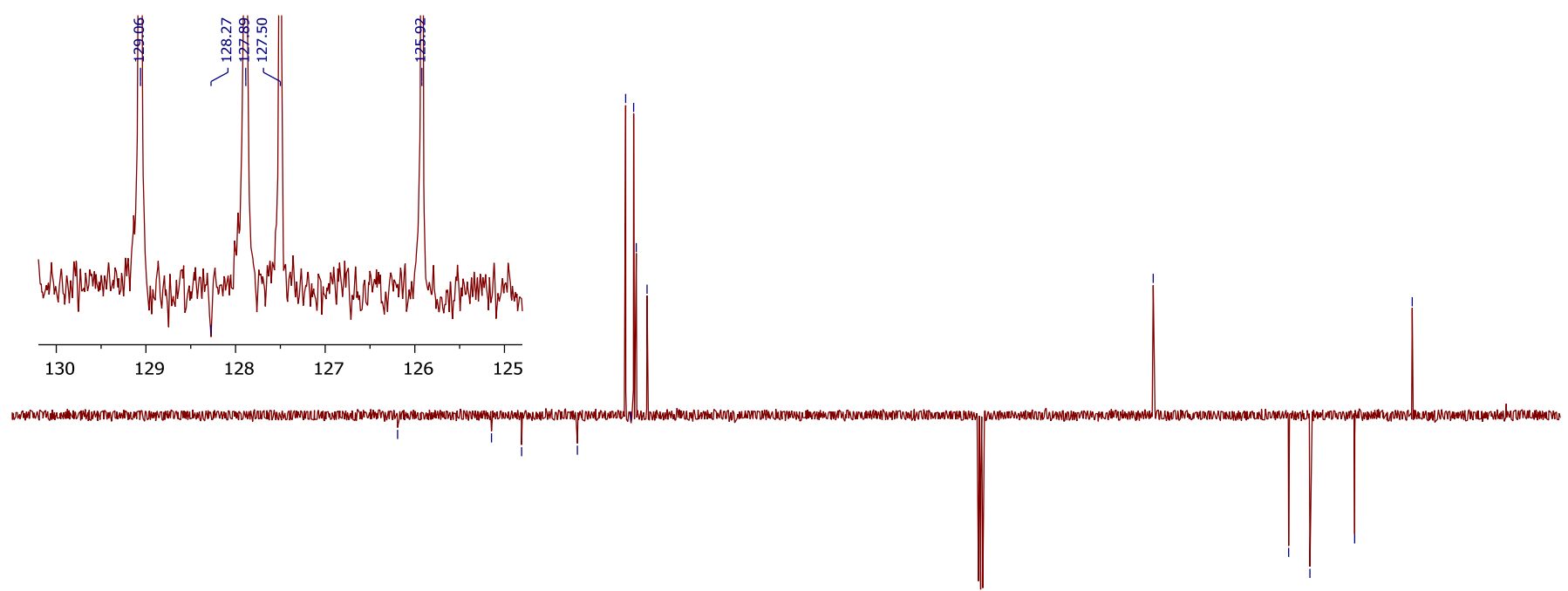

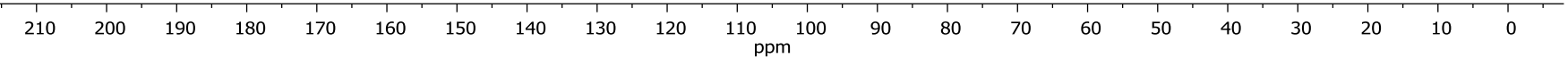



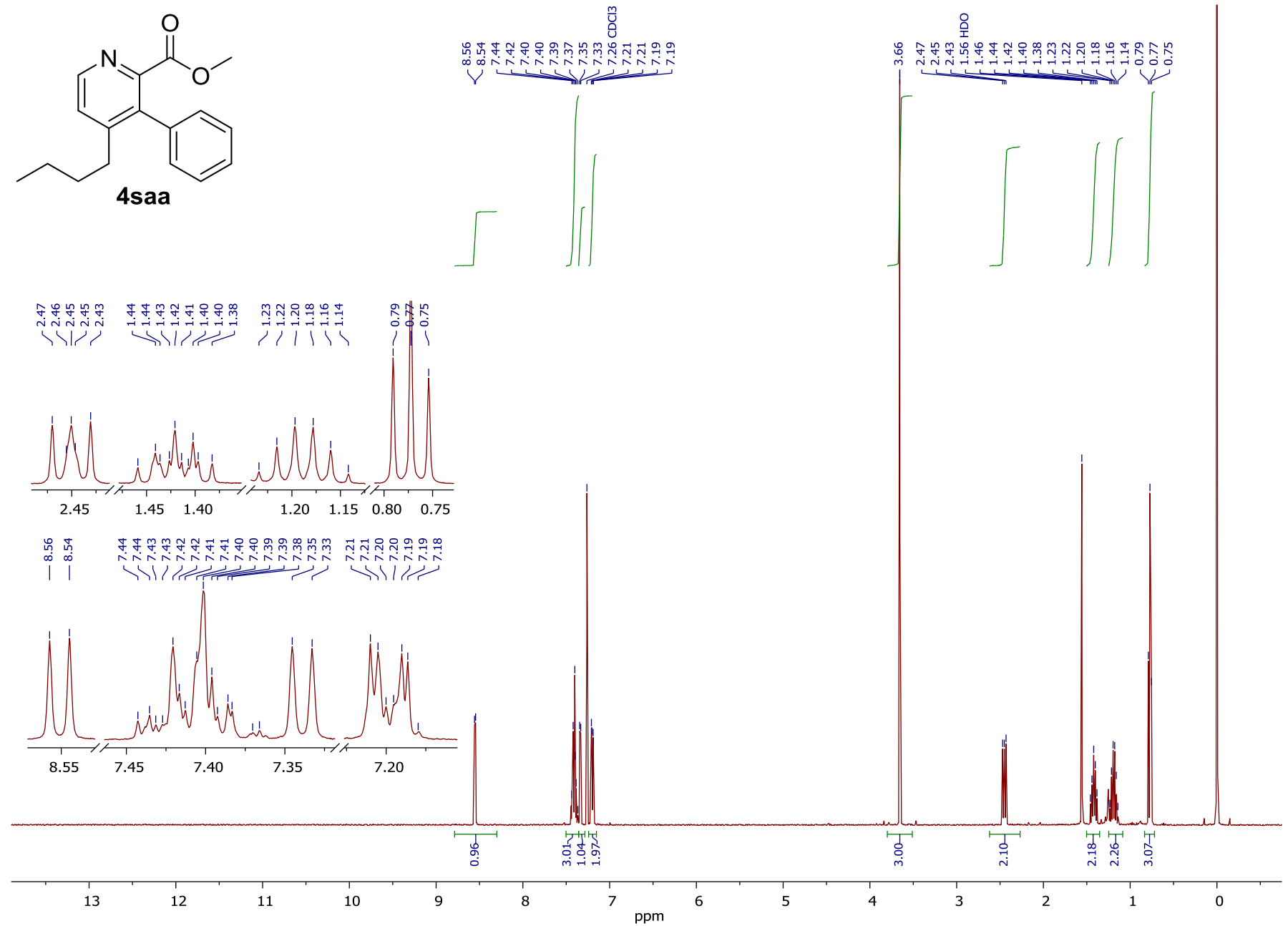

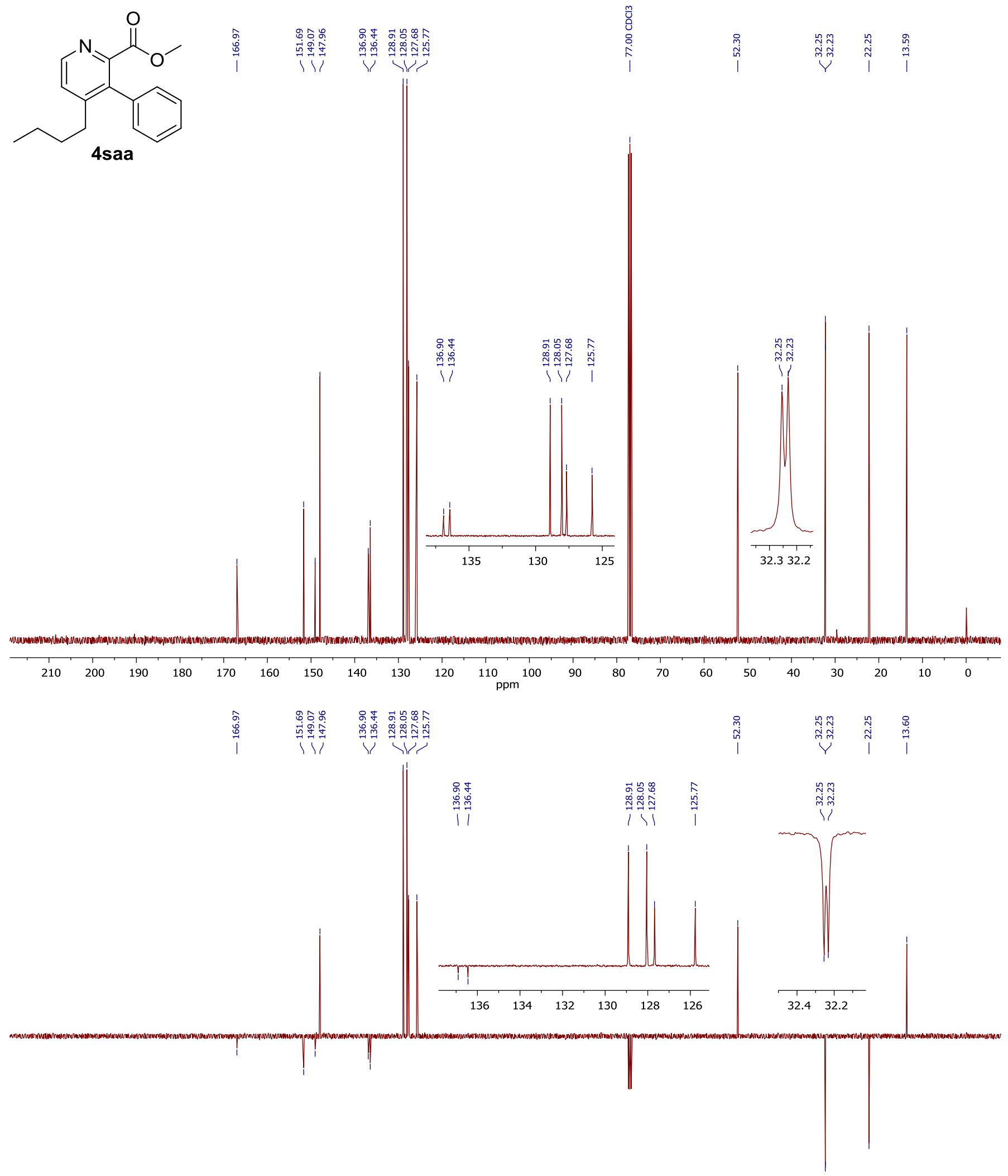

$\begin{array}{lllllllllllllllllllllll}210 & 200 & 190 & 180 & 170 & 160 & 150 & 140 & 130 & 120 & 110 & 100 & 90 & 80 & 70 & 60 & 50 & 40 & 30 & 20 & 10 & 0 & \mathrm{ppm}\end{array}$ 
<smiles>COC(=O)c1cccc(CC2CC2)c1-c1ccccc1</smiles>

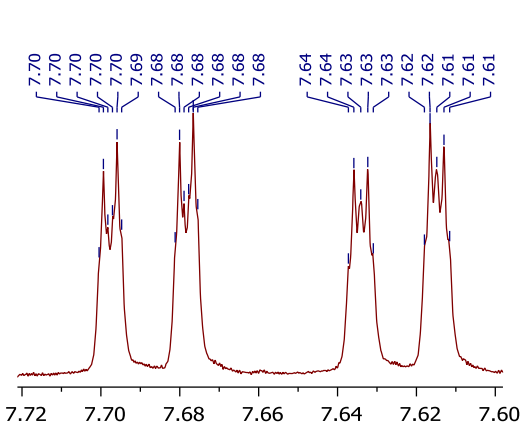

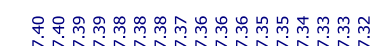
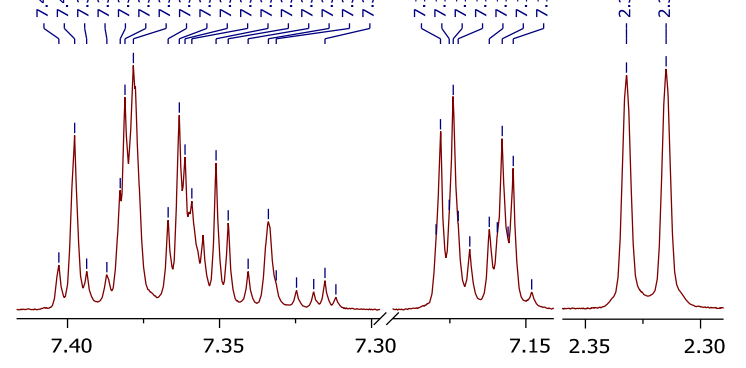

范

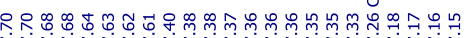

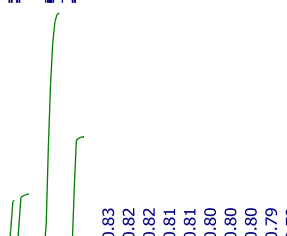

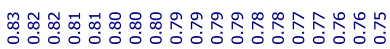
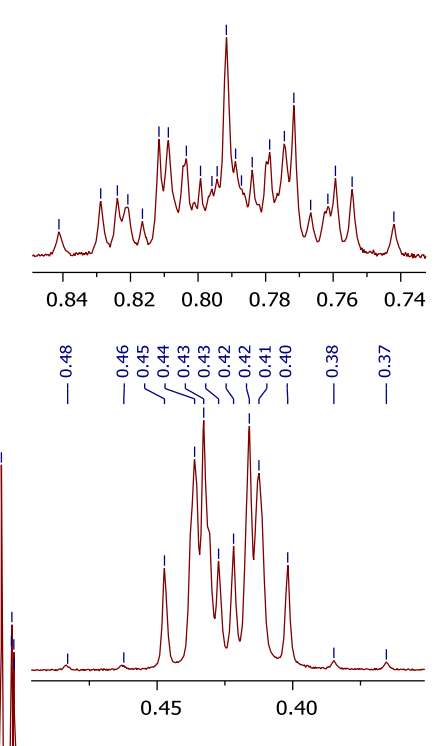

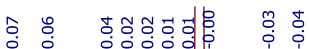
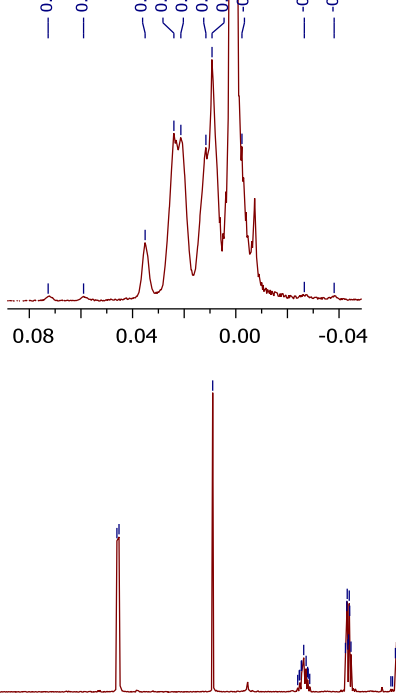

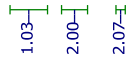

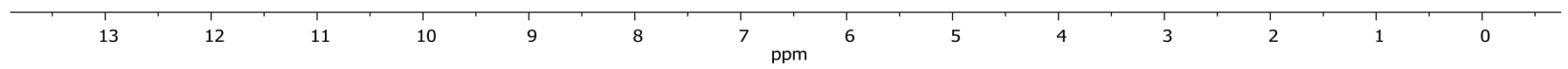



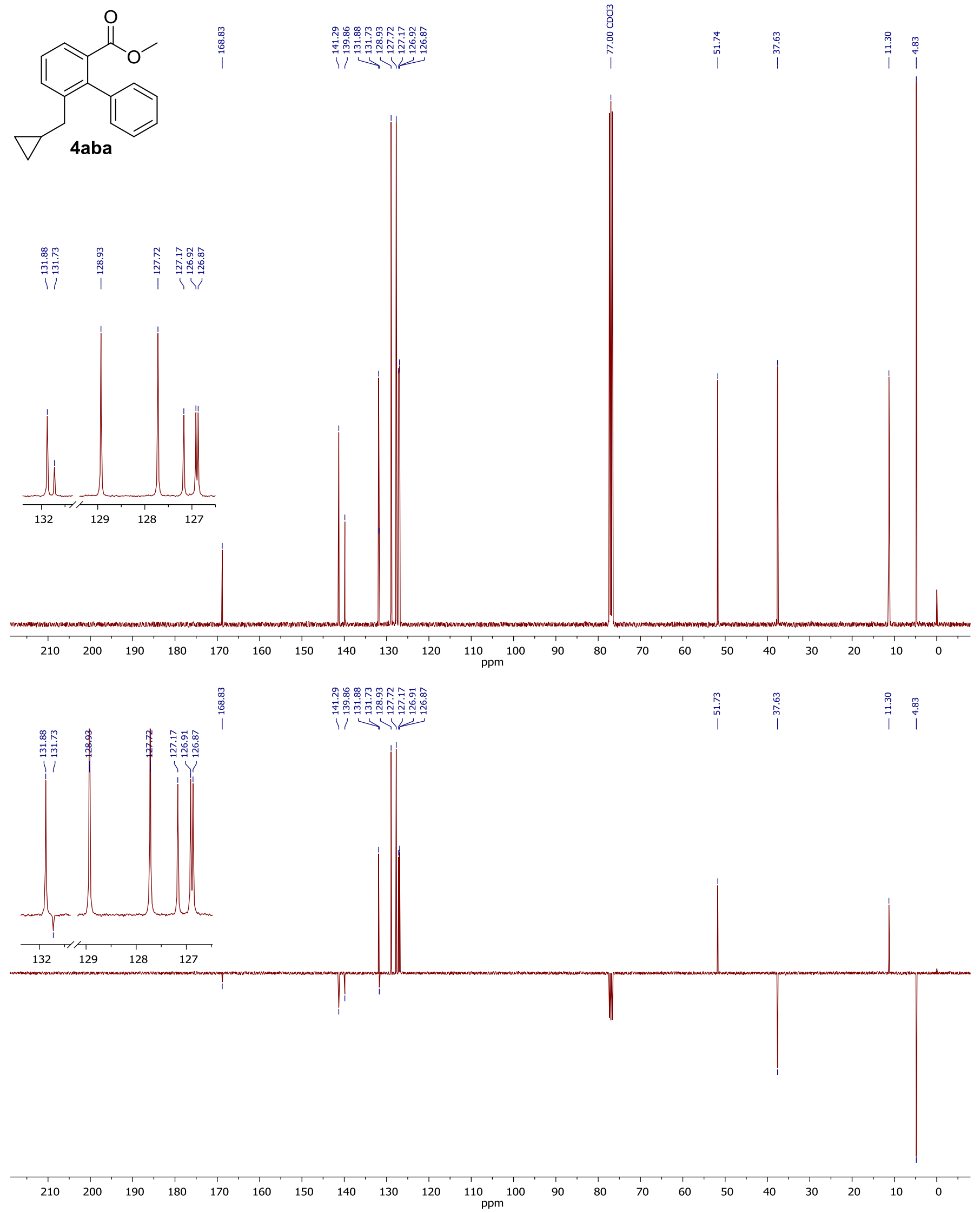
<smiles>COC(=O)c1cccc(C(C)C)c1-c1ccccc1</smiles>

4aca

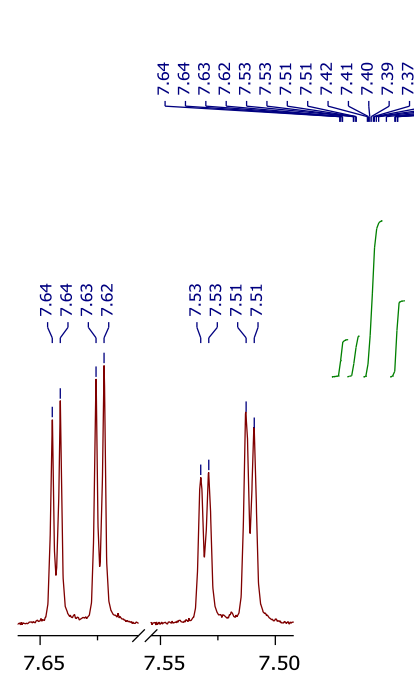

范

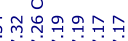

$\sin 20$
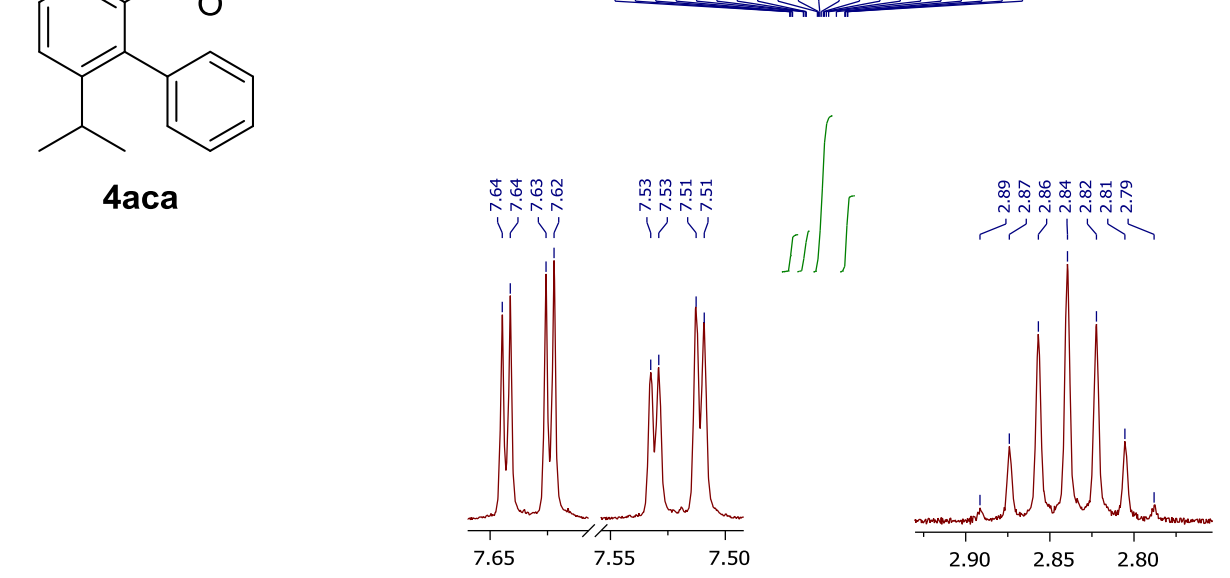

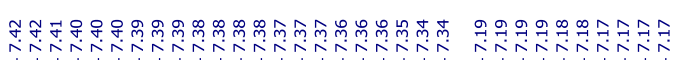

यंरोiाi,

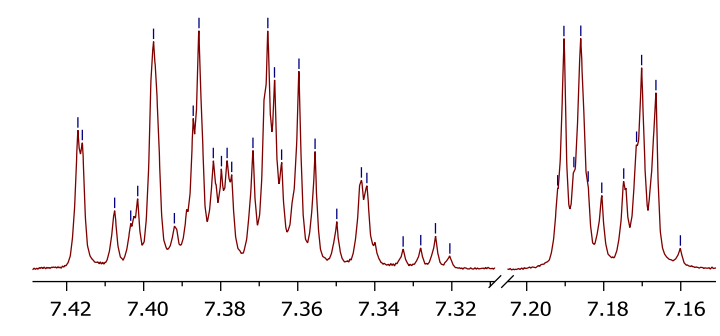

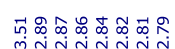

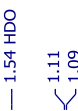

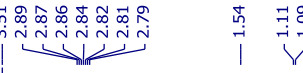

궁

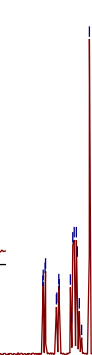

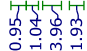

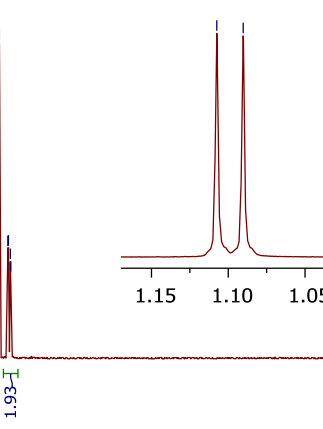

$\begin{array}{lllllllll}13 & 12 & 11 & 10 & 9 & 8 & 7 & \text { ppm }\end{array}$

$\begin{array}{lll}6 & 5 & 4\end{array}$

3

(1)

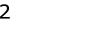
ד 

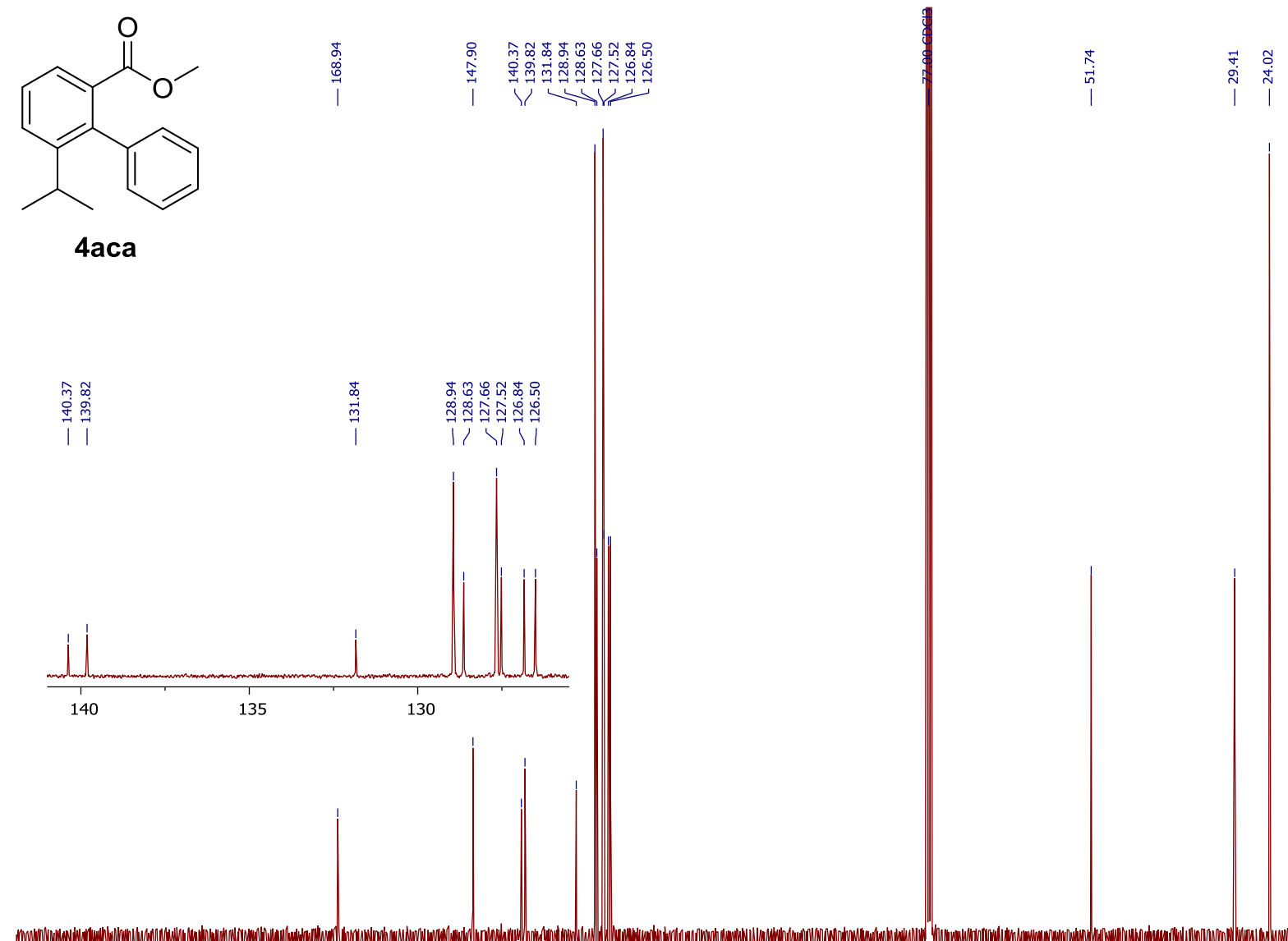

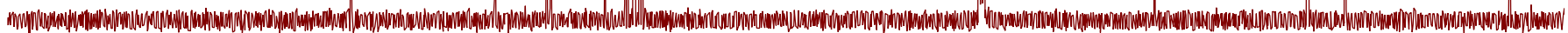

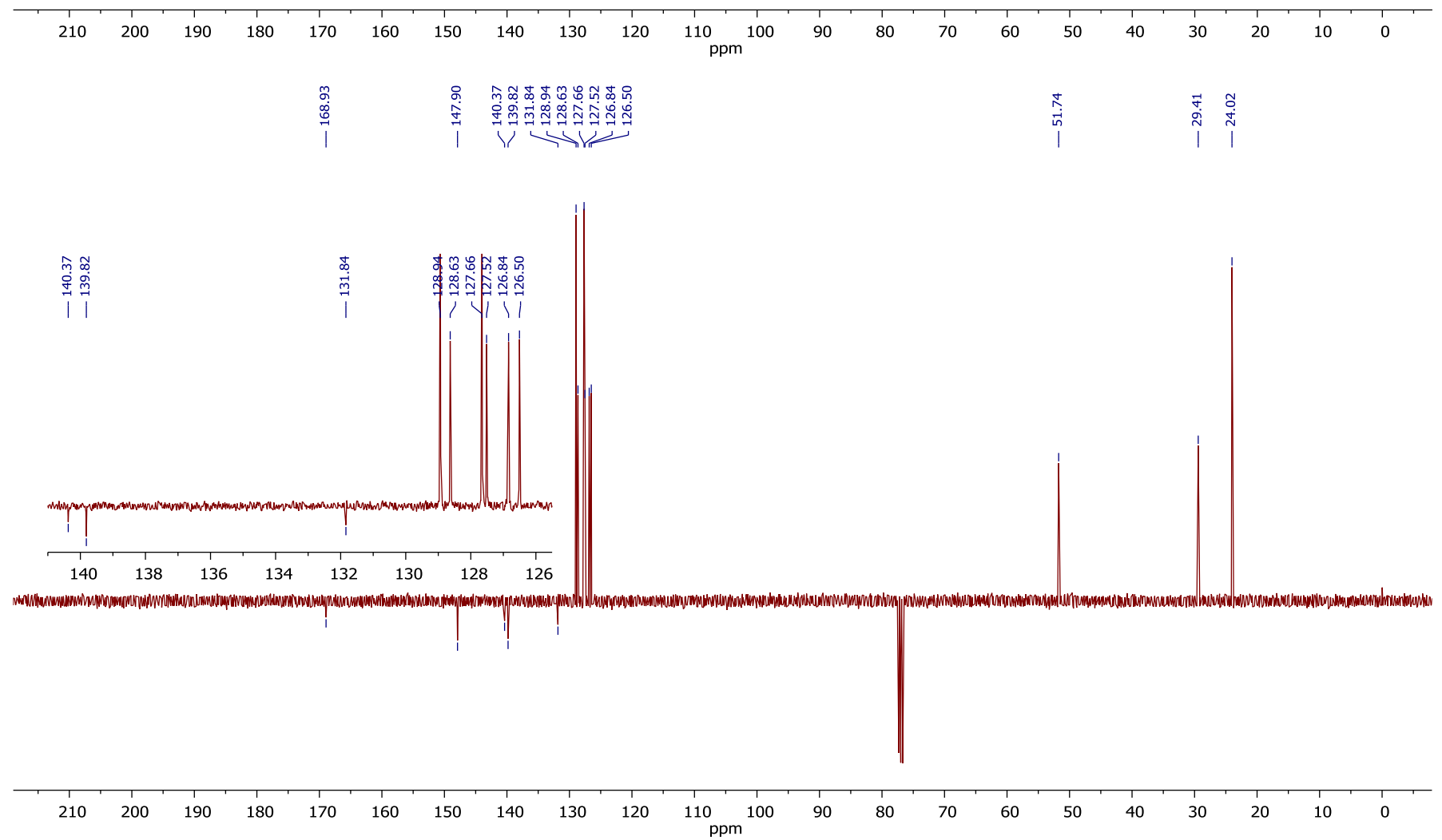




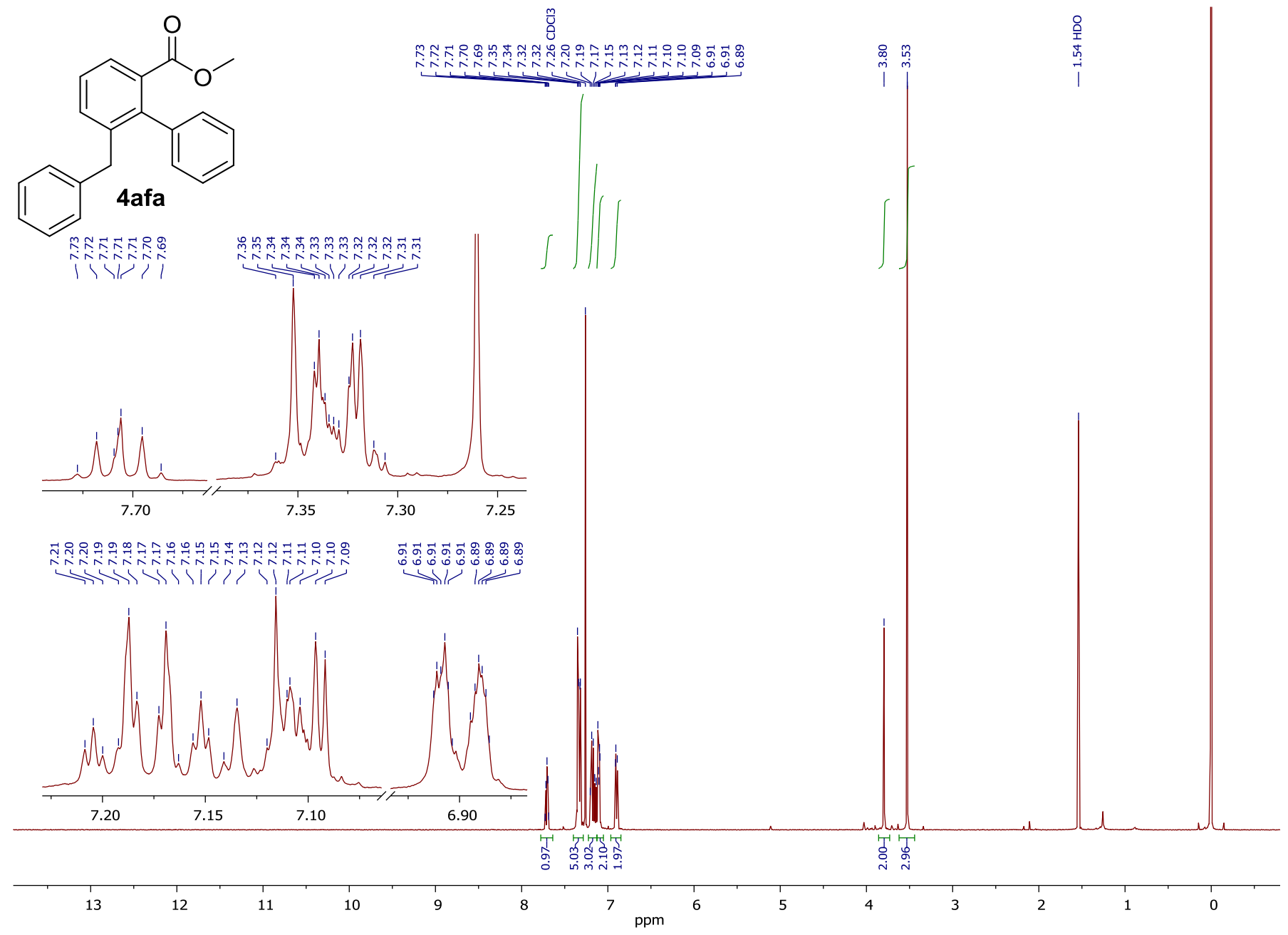


<smiles>COC(=O)c1cccc(Cc2ccccc2)c1-c1ccccc1</smiles>
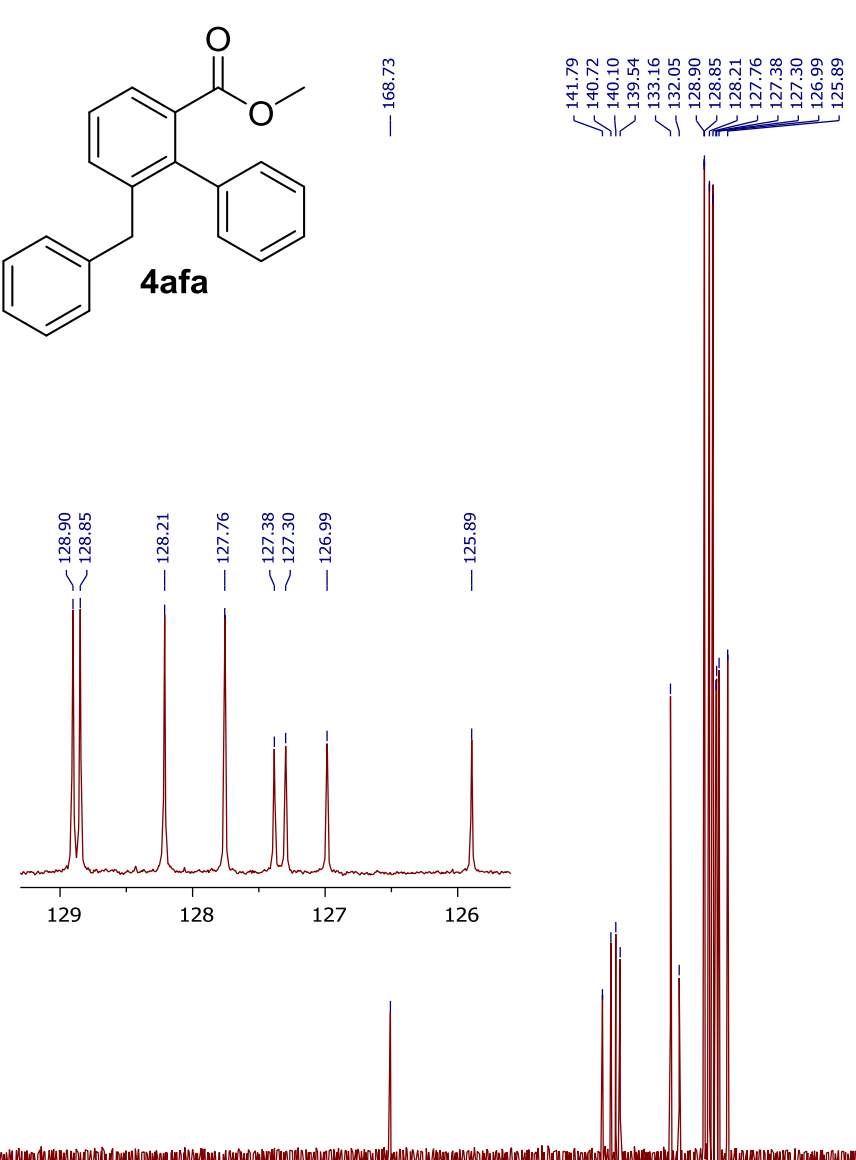

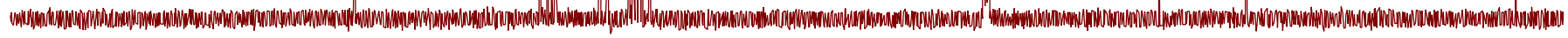

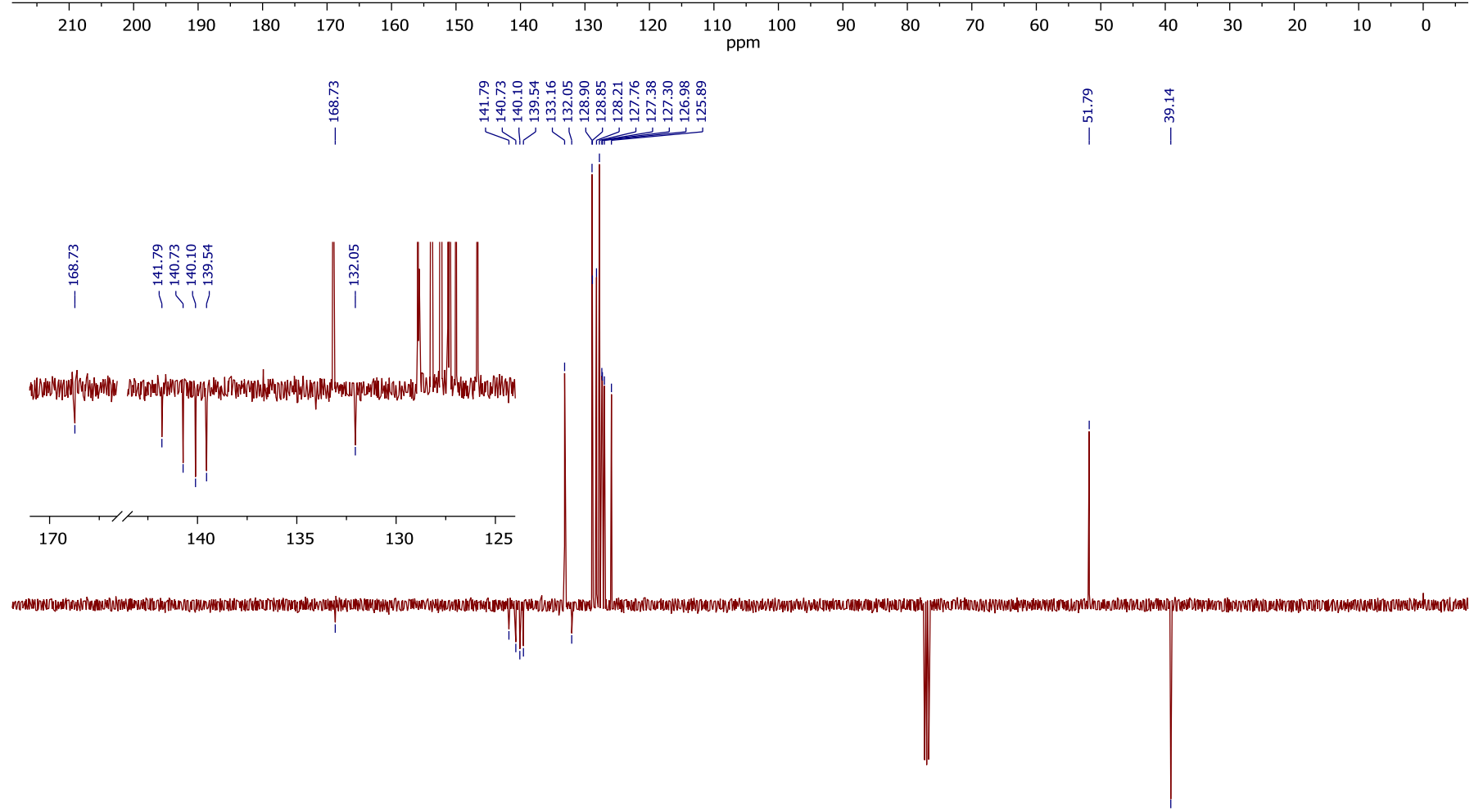

$\begin{array}{lllllllllllllllllllllll} & 210 & 200 & 190 & 180 & 170 & 160 & 150 & 140 & 130 & 120 & 110 & 100 & 90 & 80 & 70 & 60 & 50 & 40 & 30 & 20 & 10 & 0\end{array}$




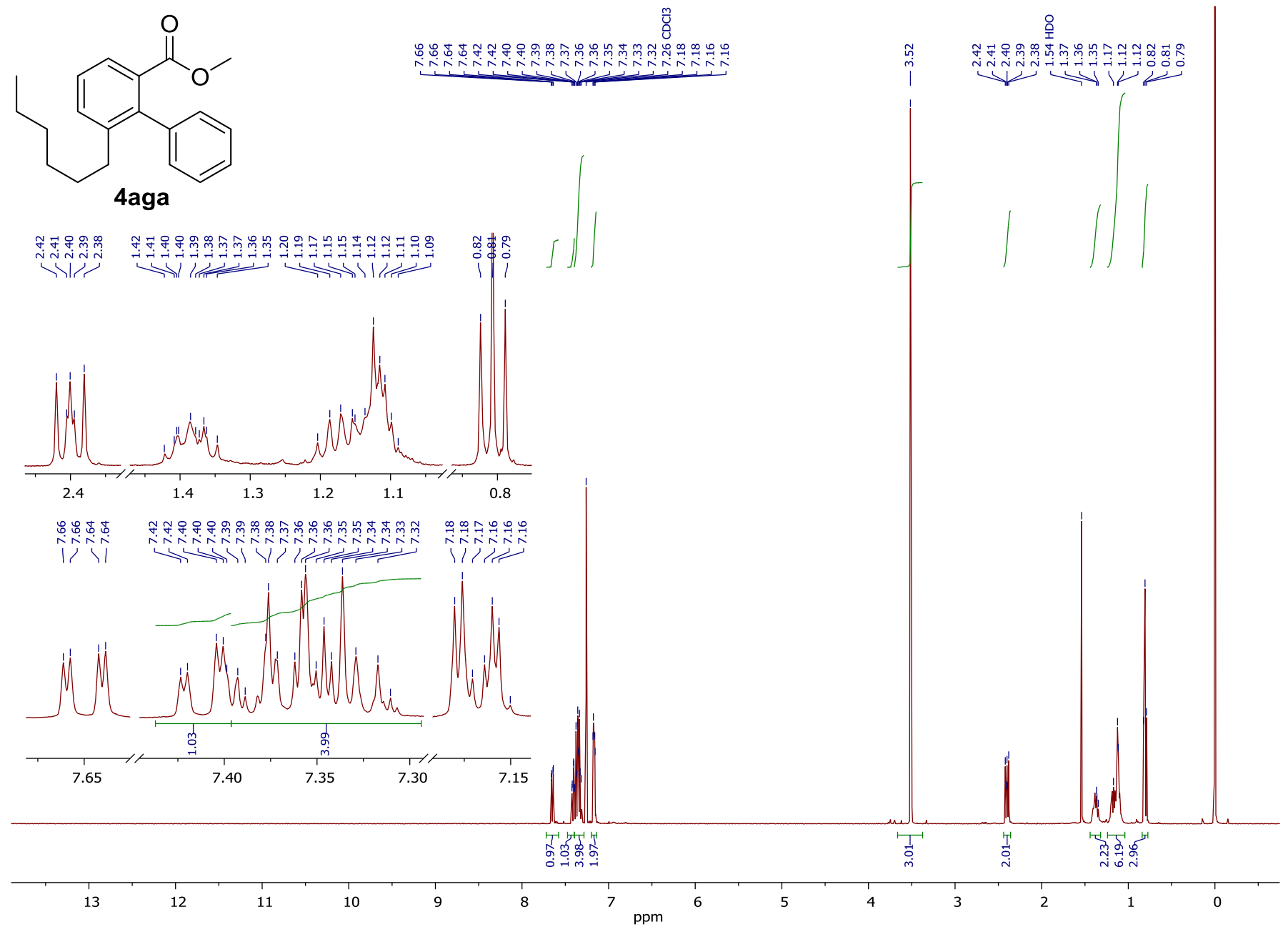



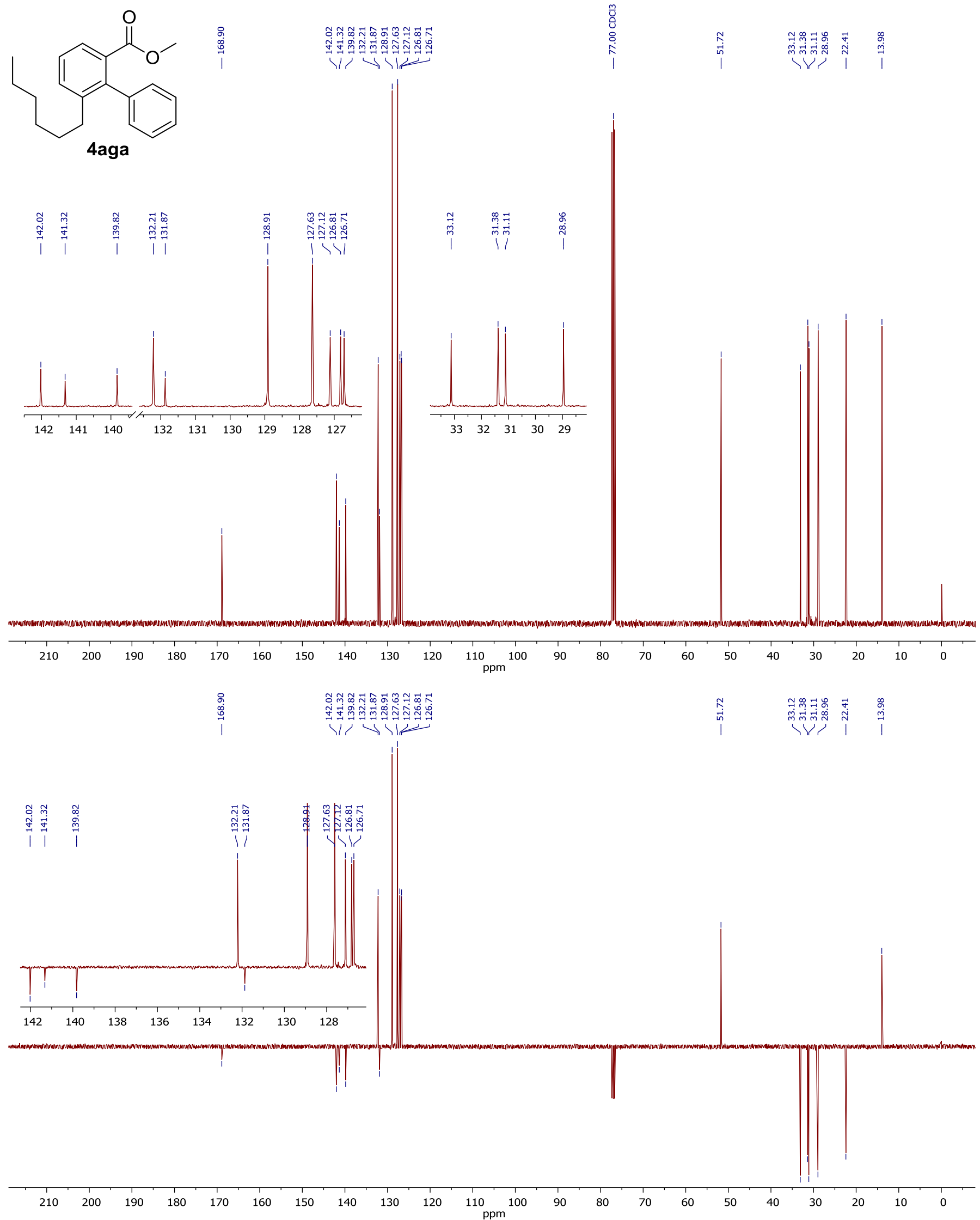
<smiles>CCCCc1cccc(C(=O)OC)c1-c1ccc(OC)cc1</smiles>

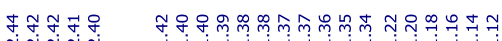

Nili

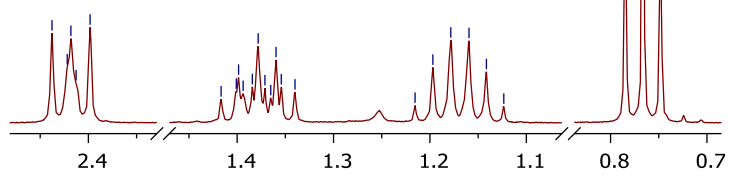

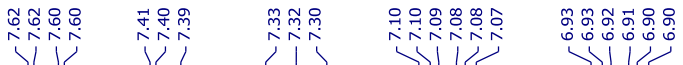

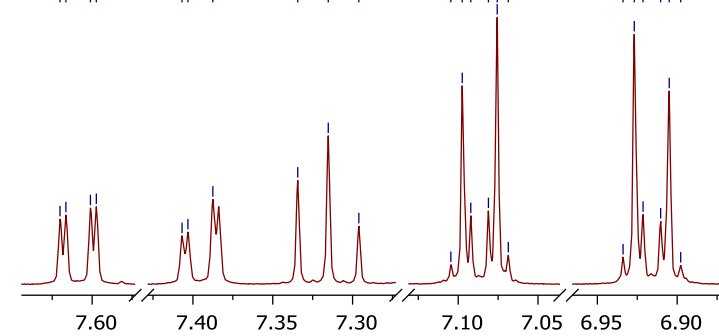

㩊

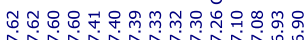

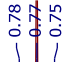

$\iiint$

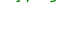

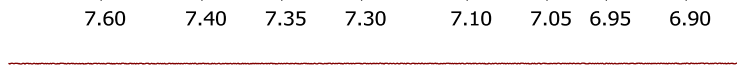

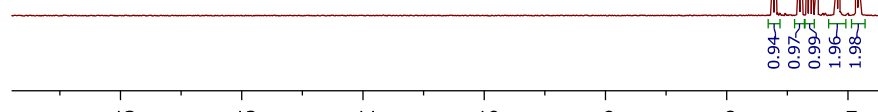

$13 \quad 12 \quad 11$

10
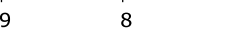

7 ppm

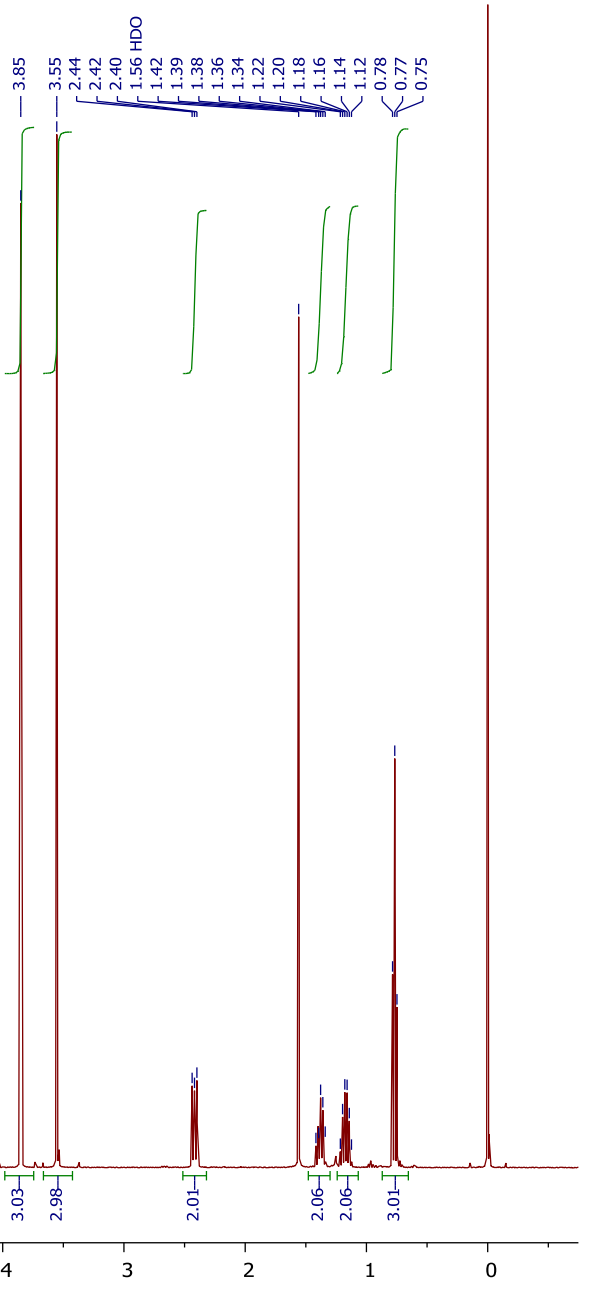




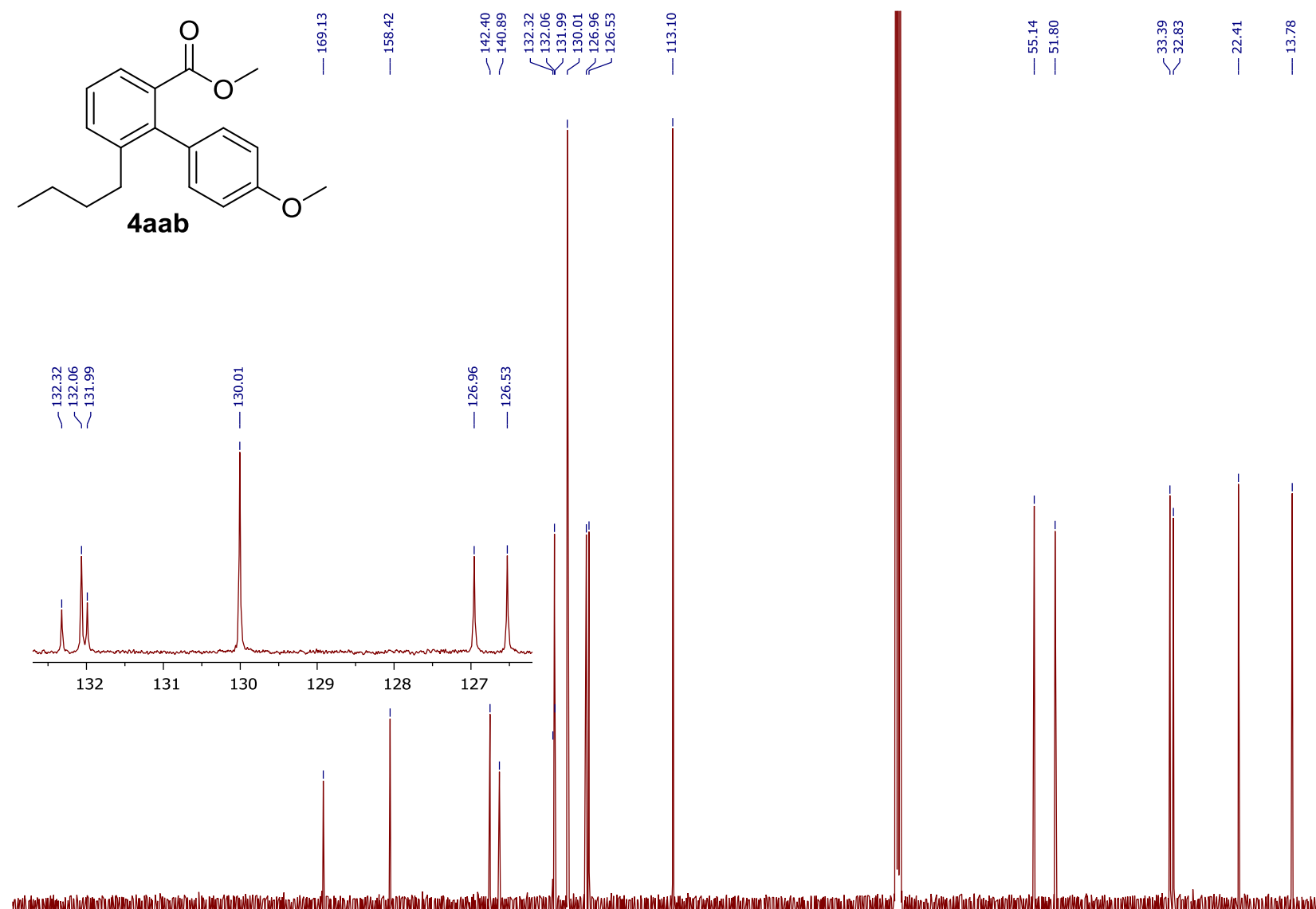

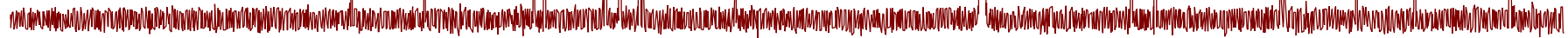

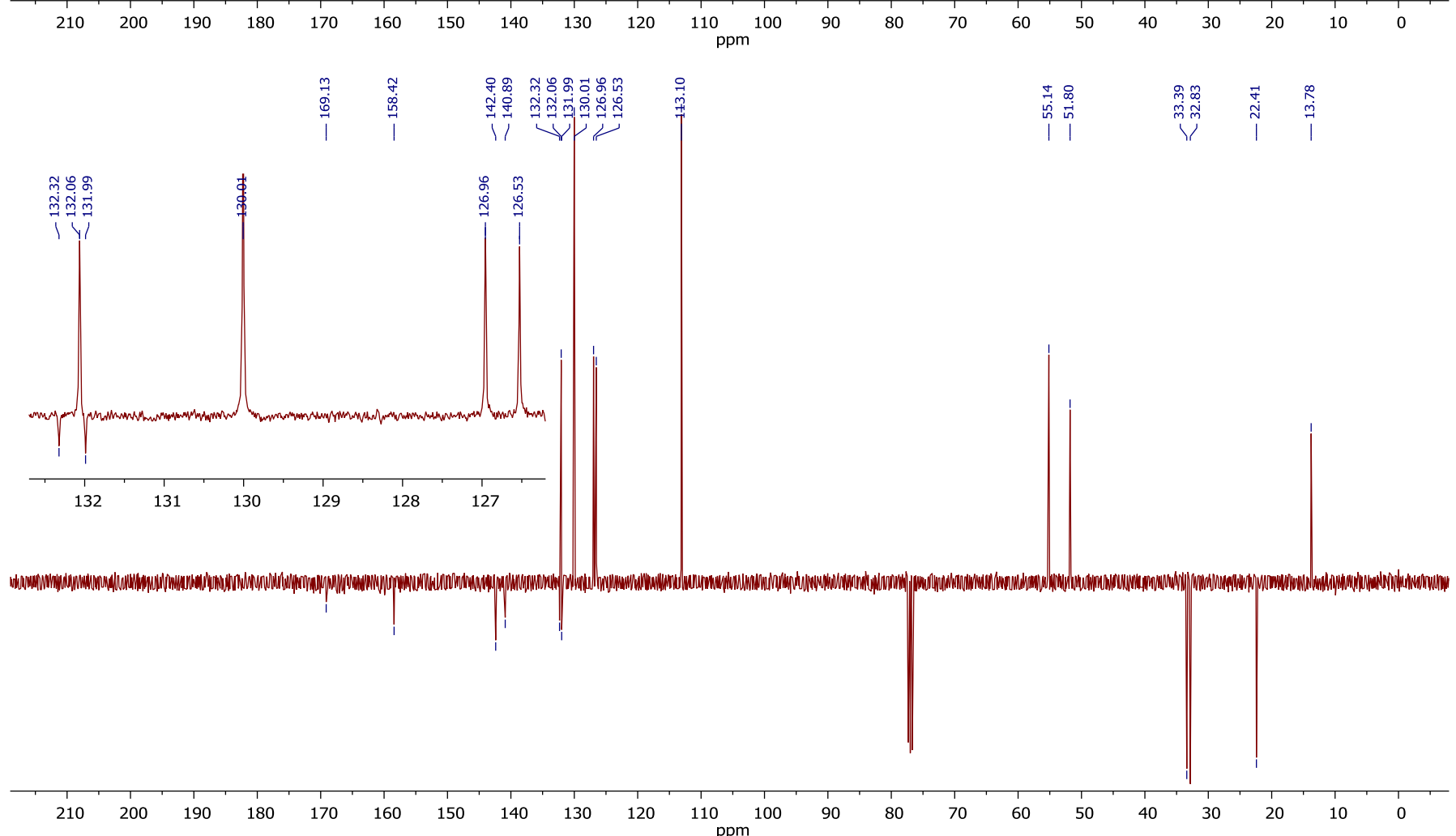



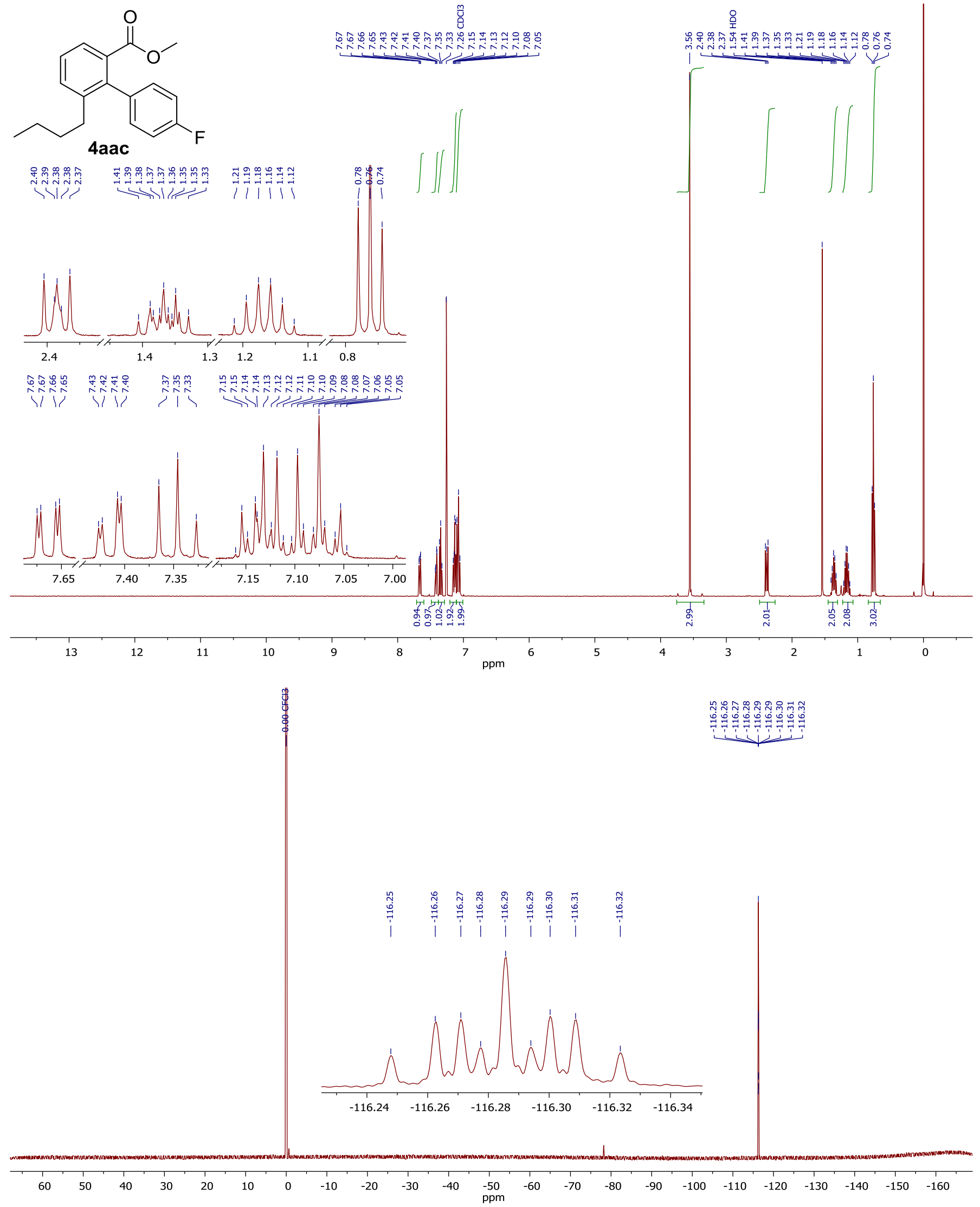

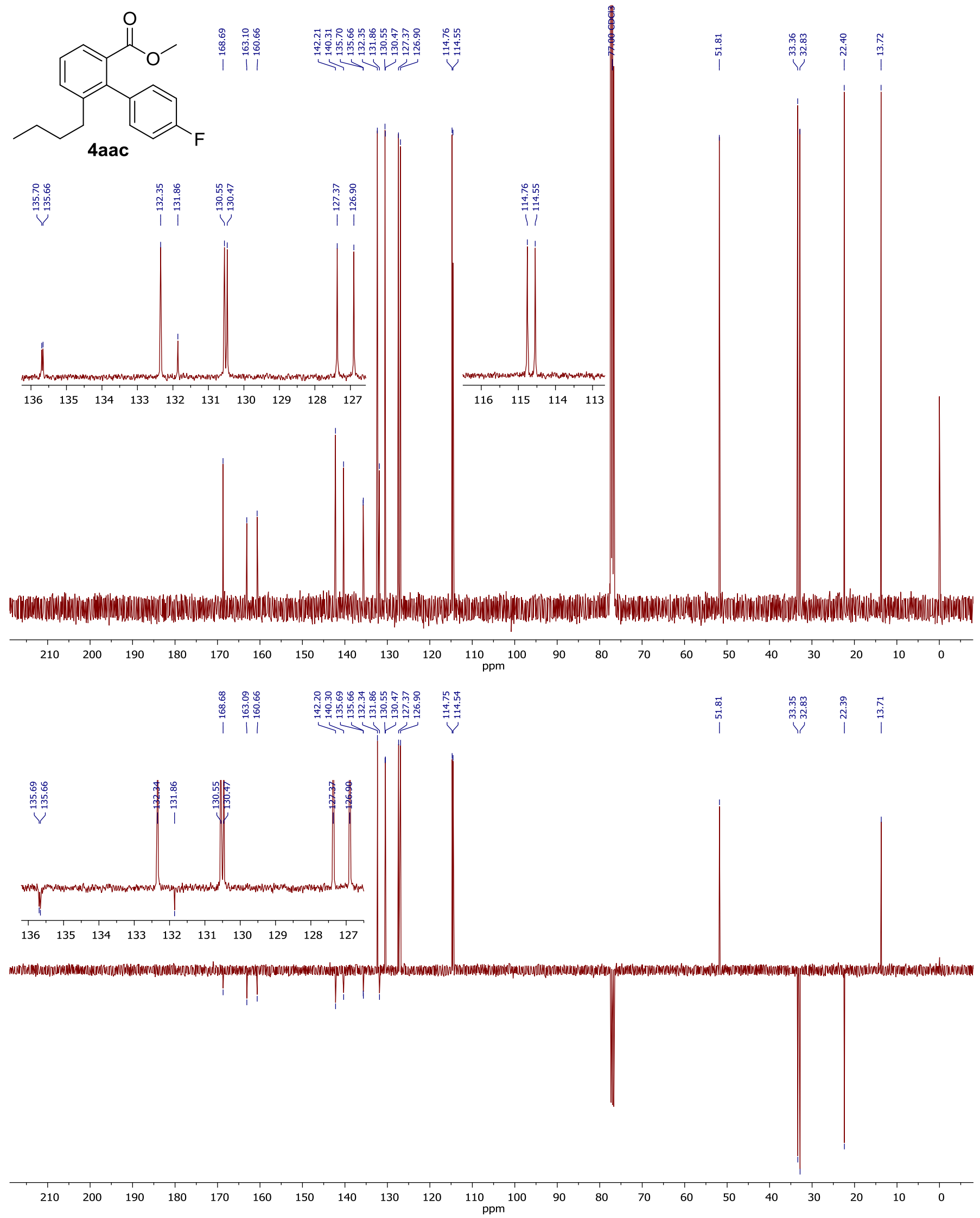


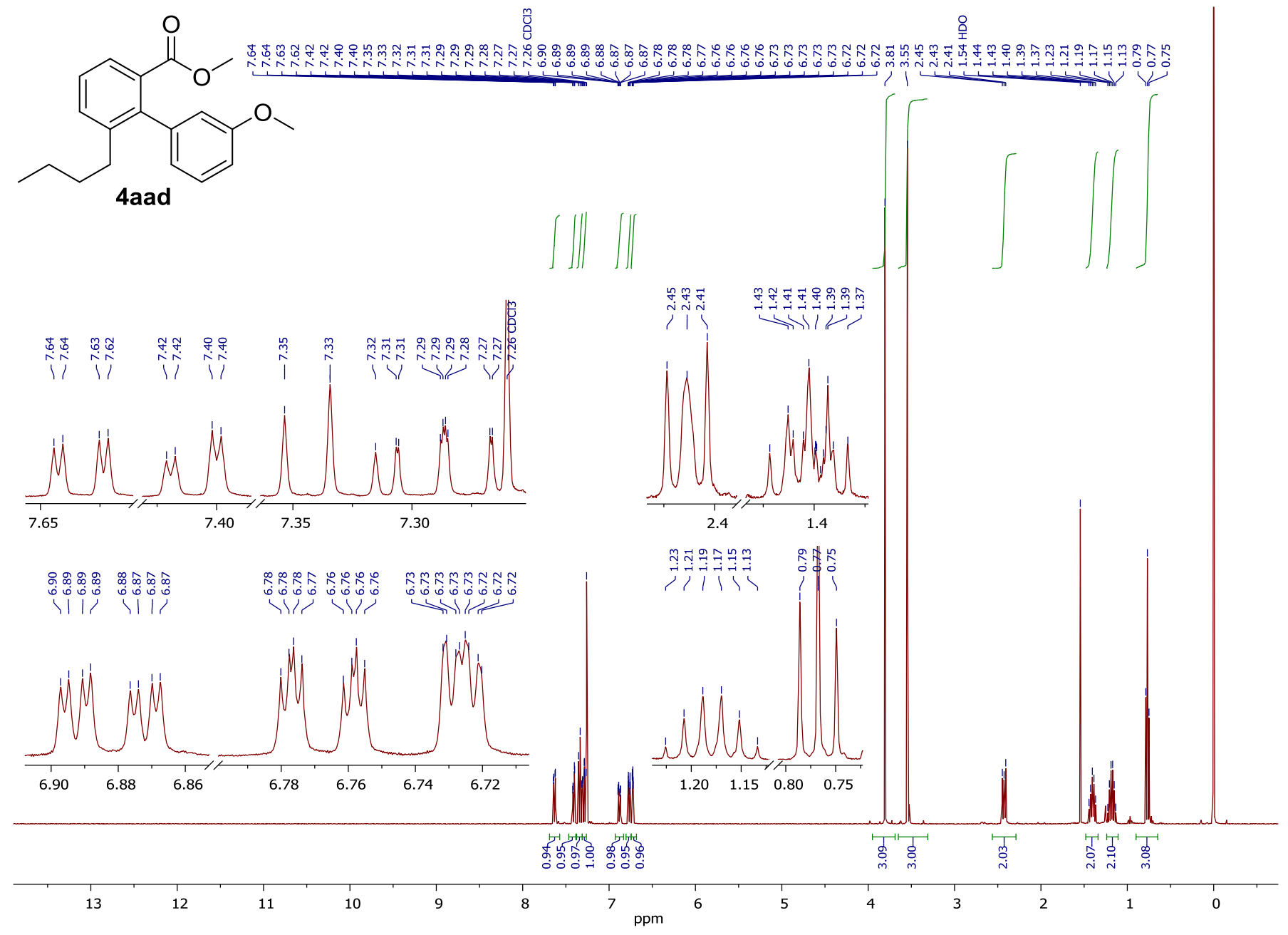



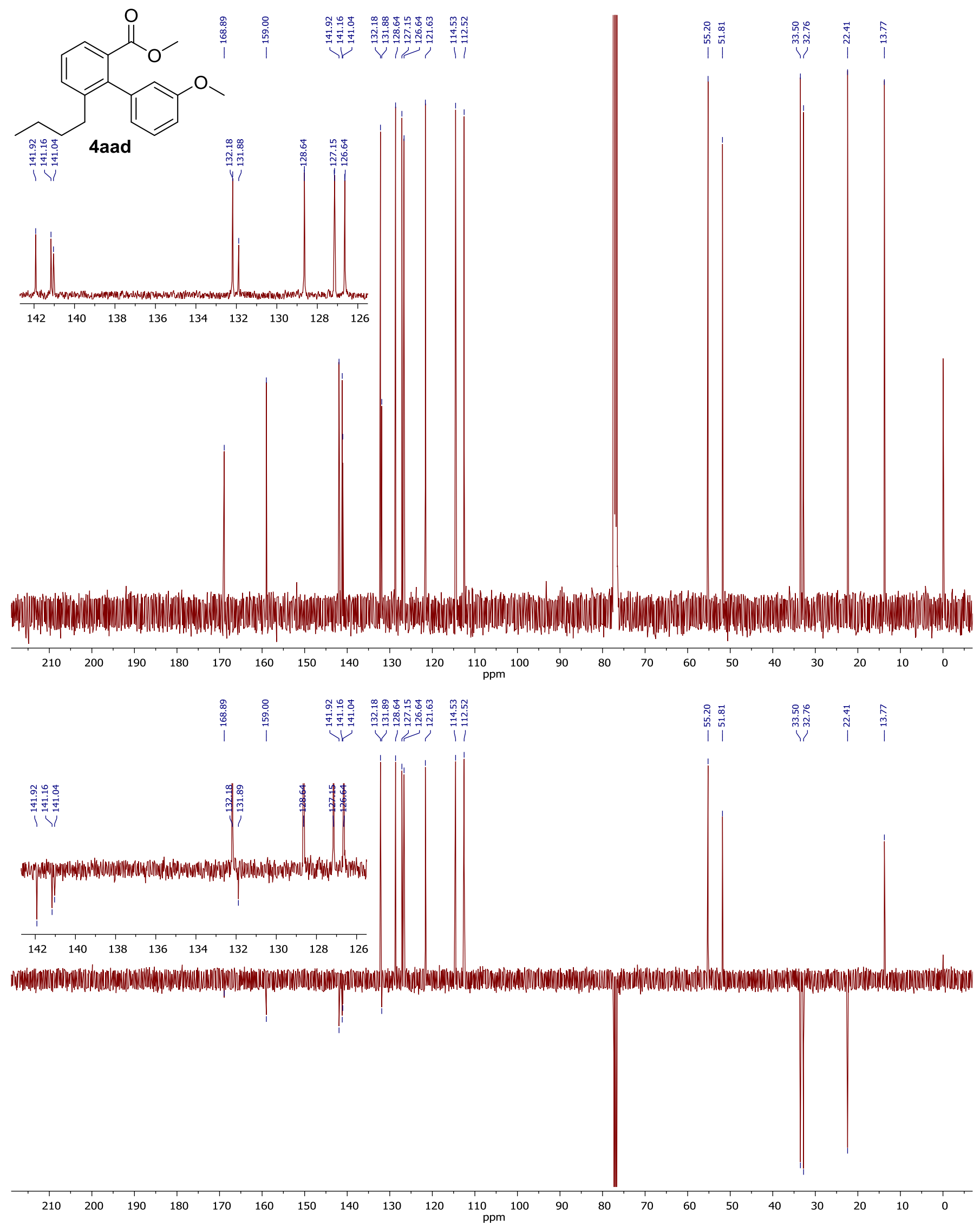

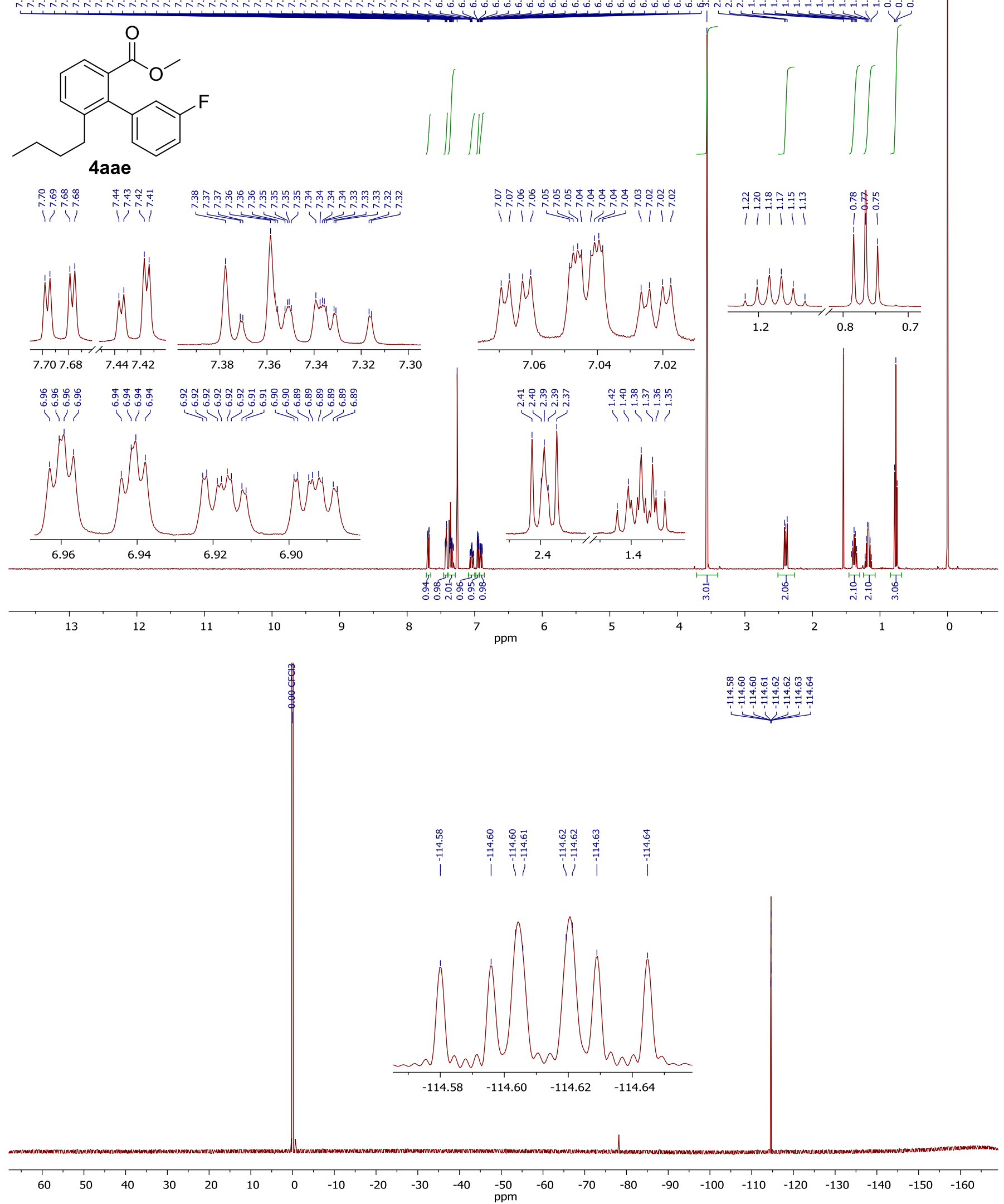

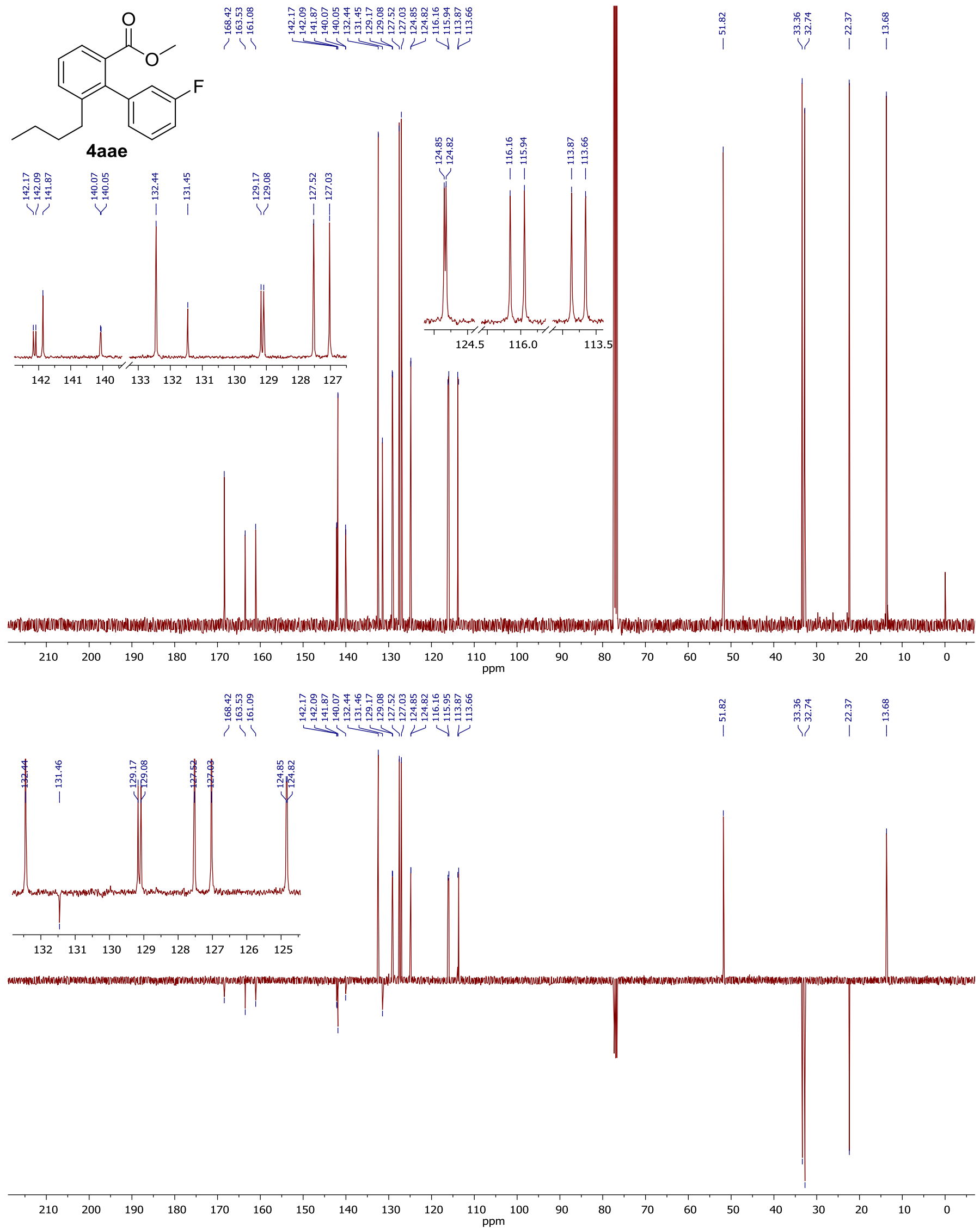
<smiles>CCCCc1cccc(C(=O)OC)c1-c1cccc(Cl)c1</smiles>

îi

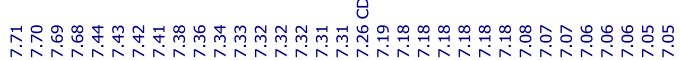

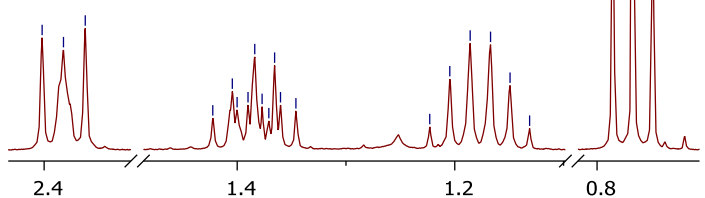

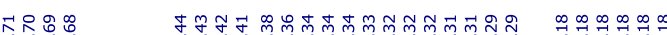

ii
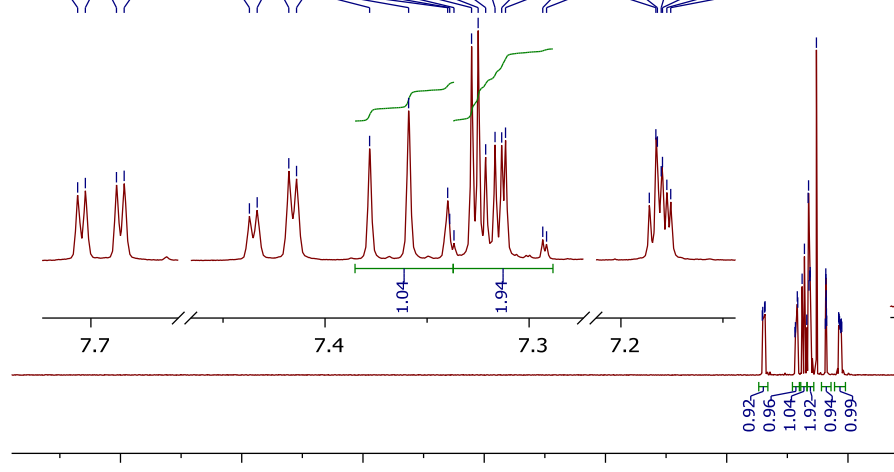

13

12

11

10

舟

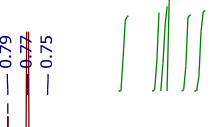

웅

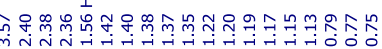

111111

$\iint$
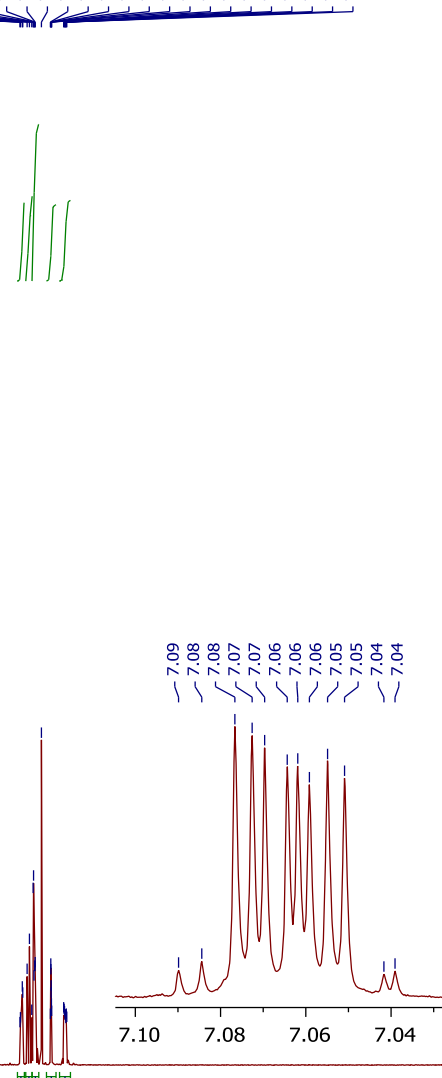

$\mathrm{ppm}$ 

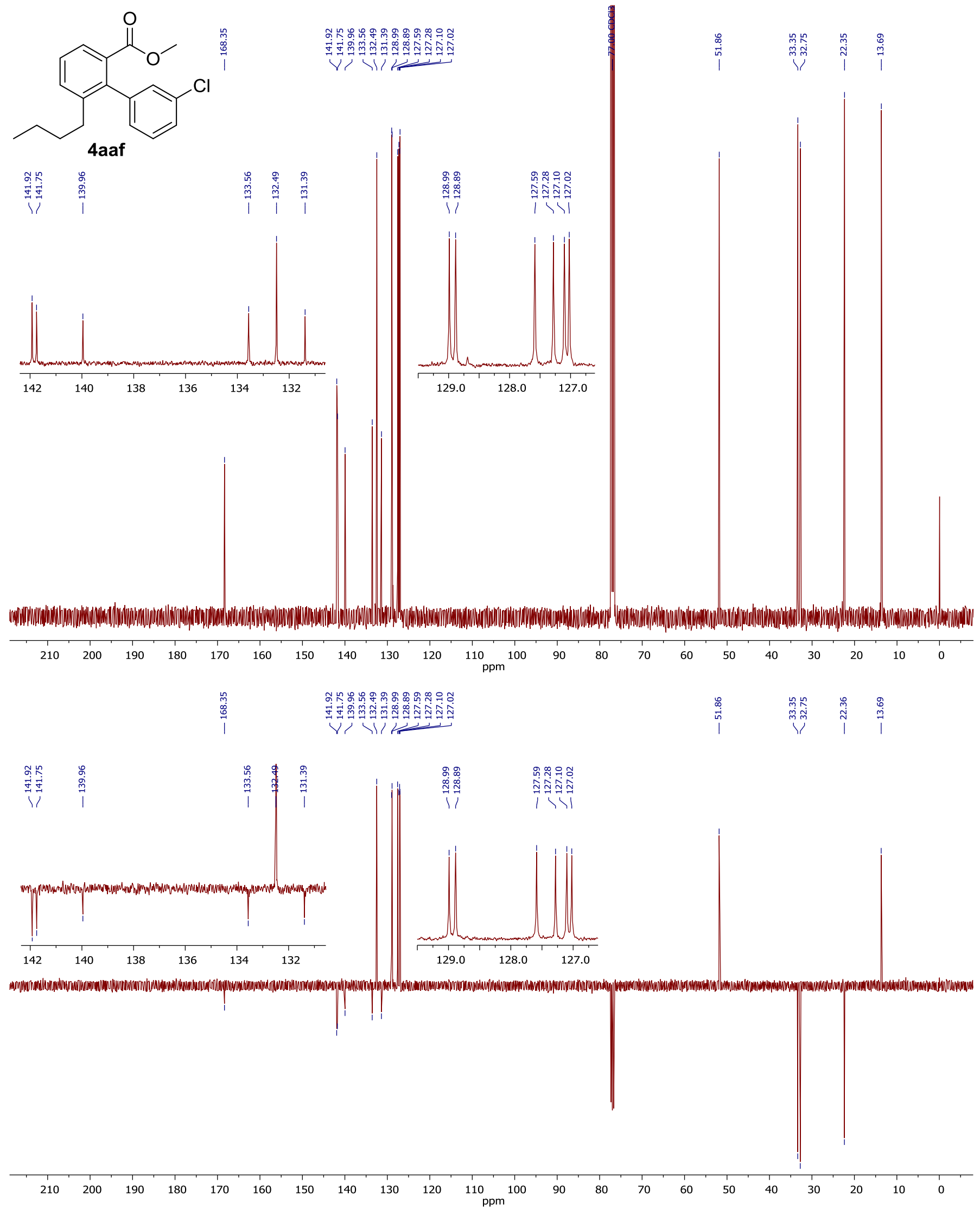


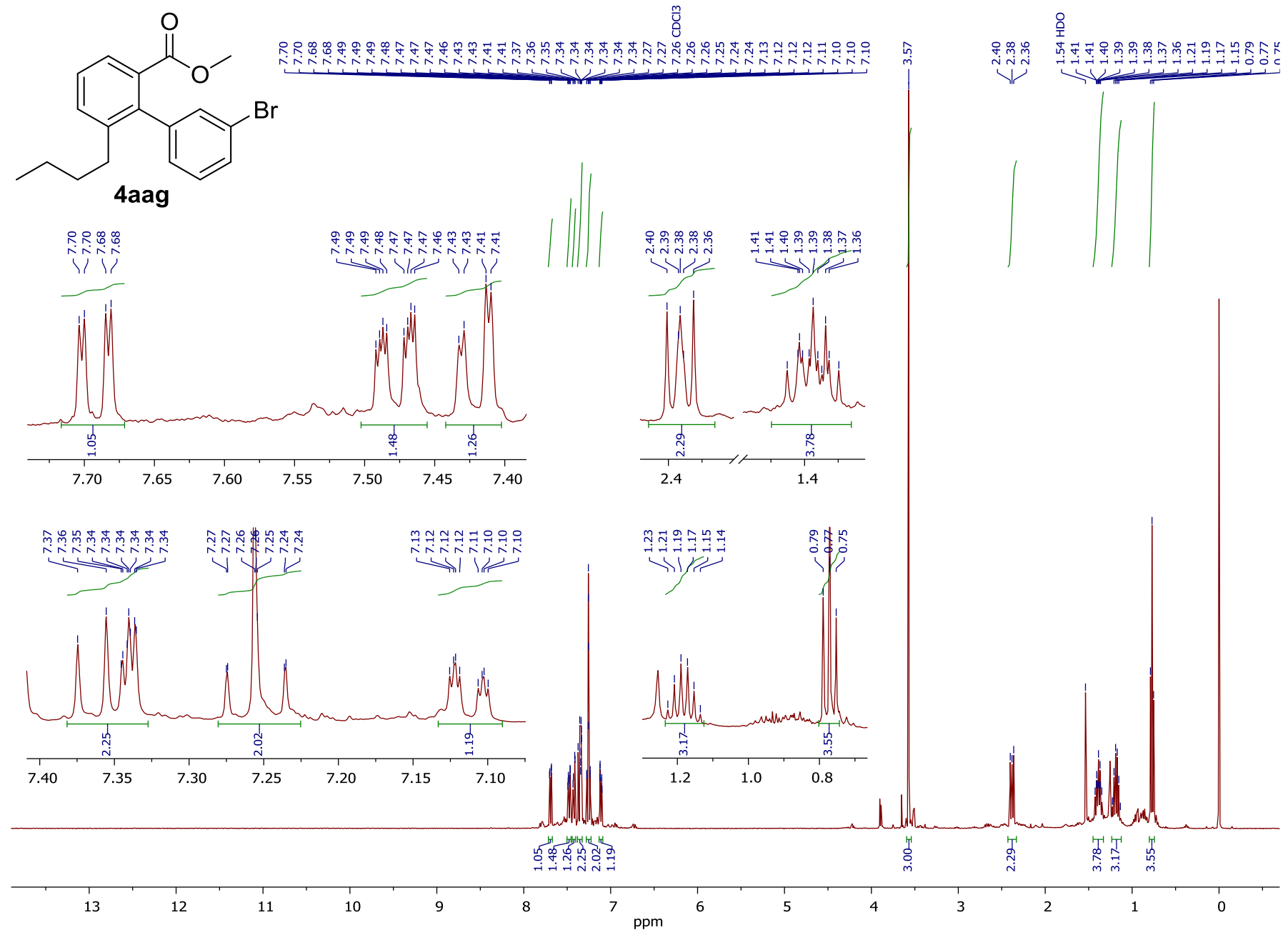



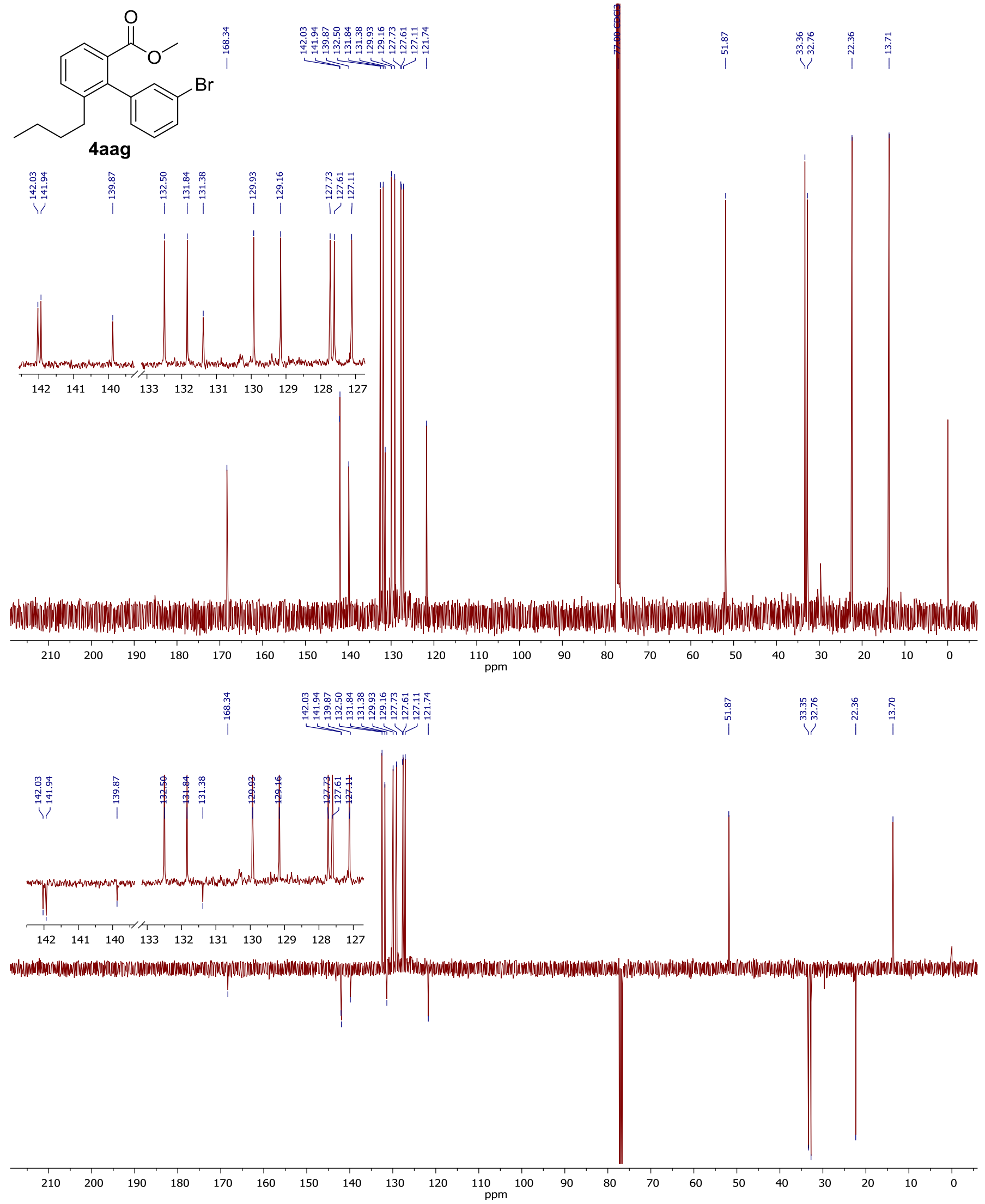


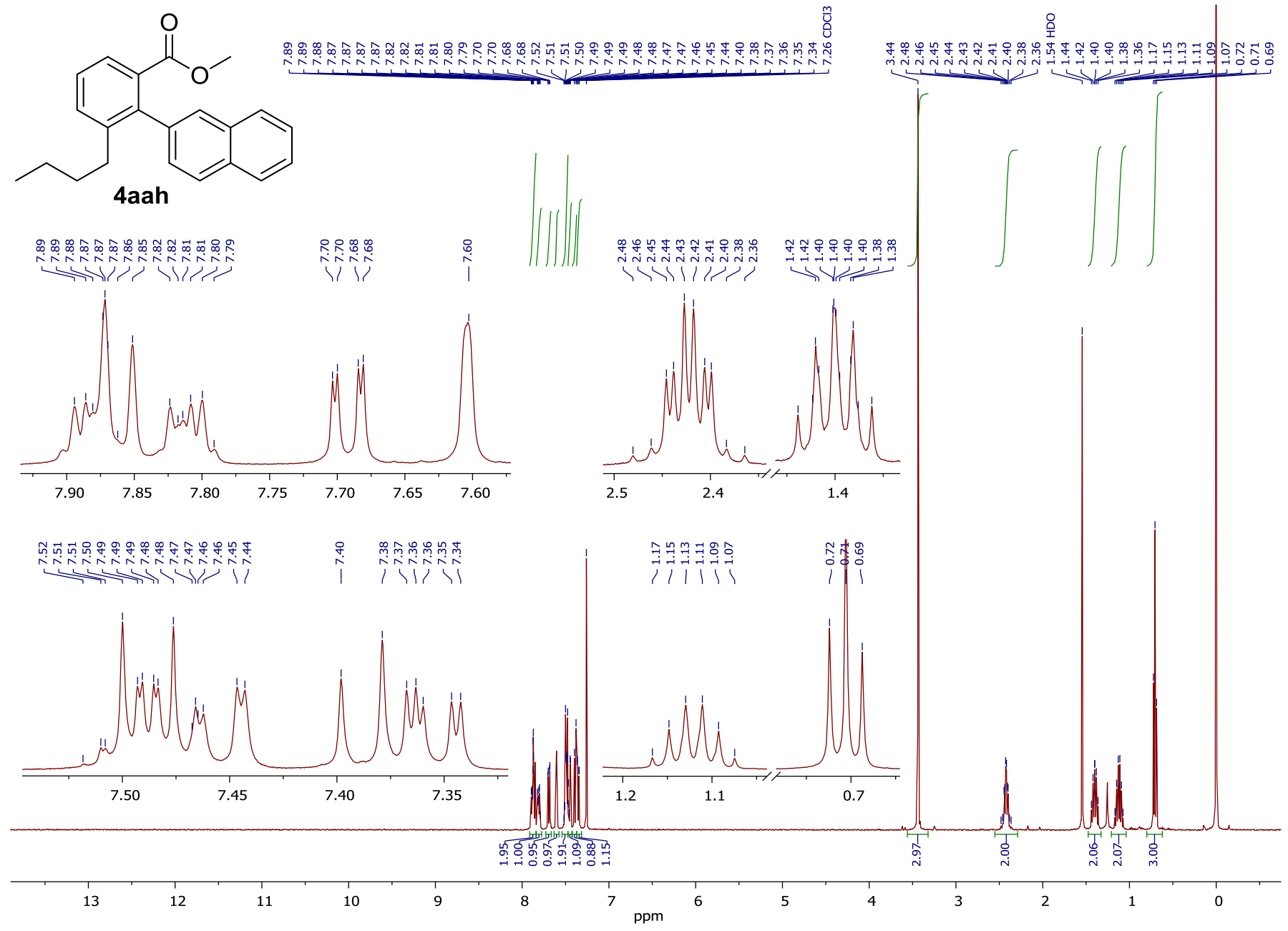



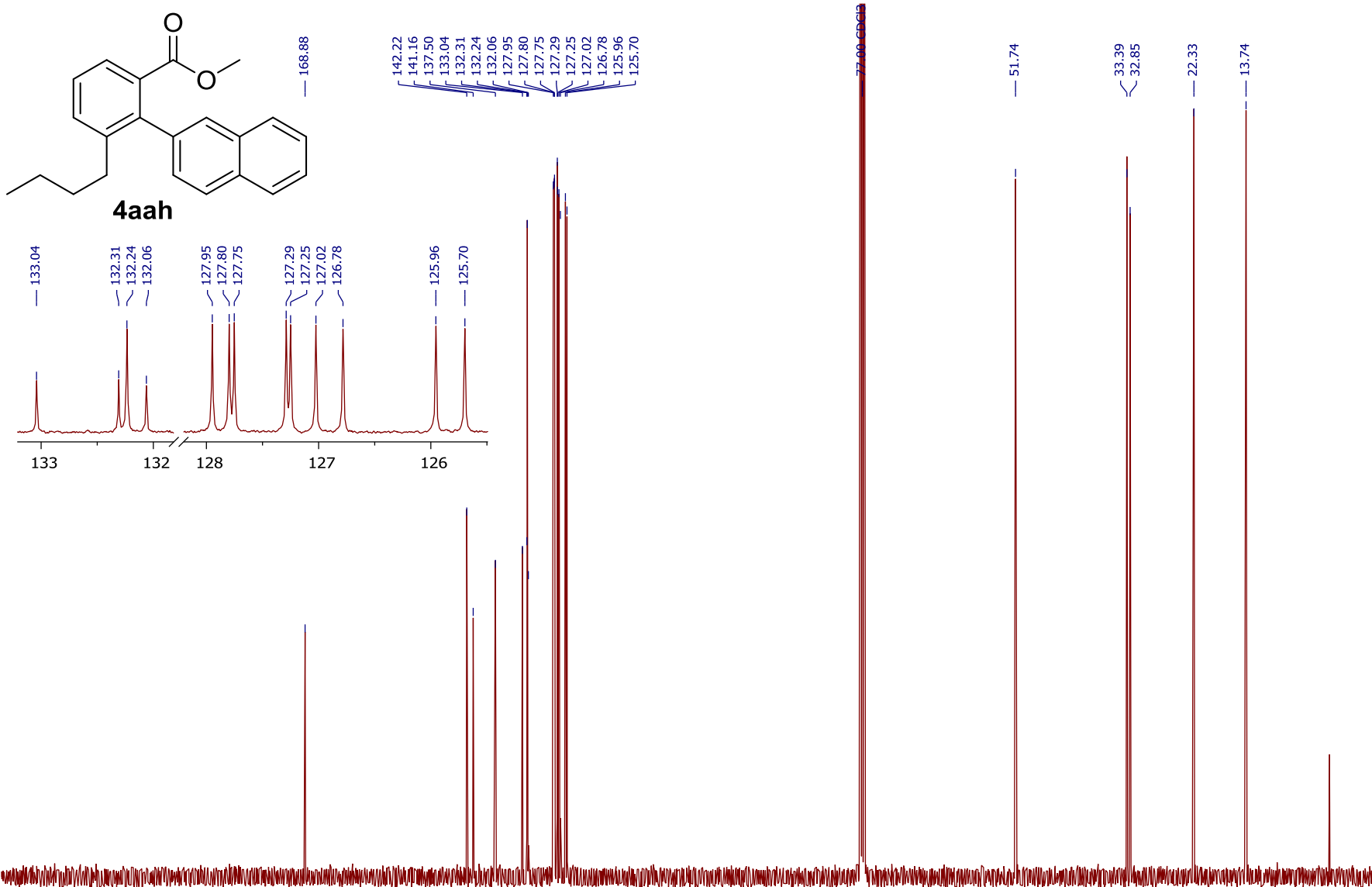

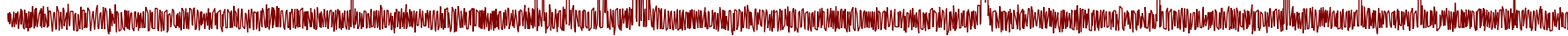

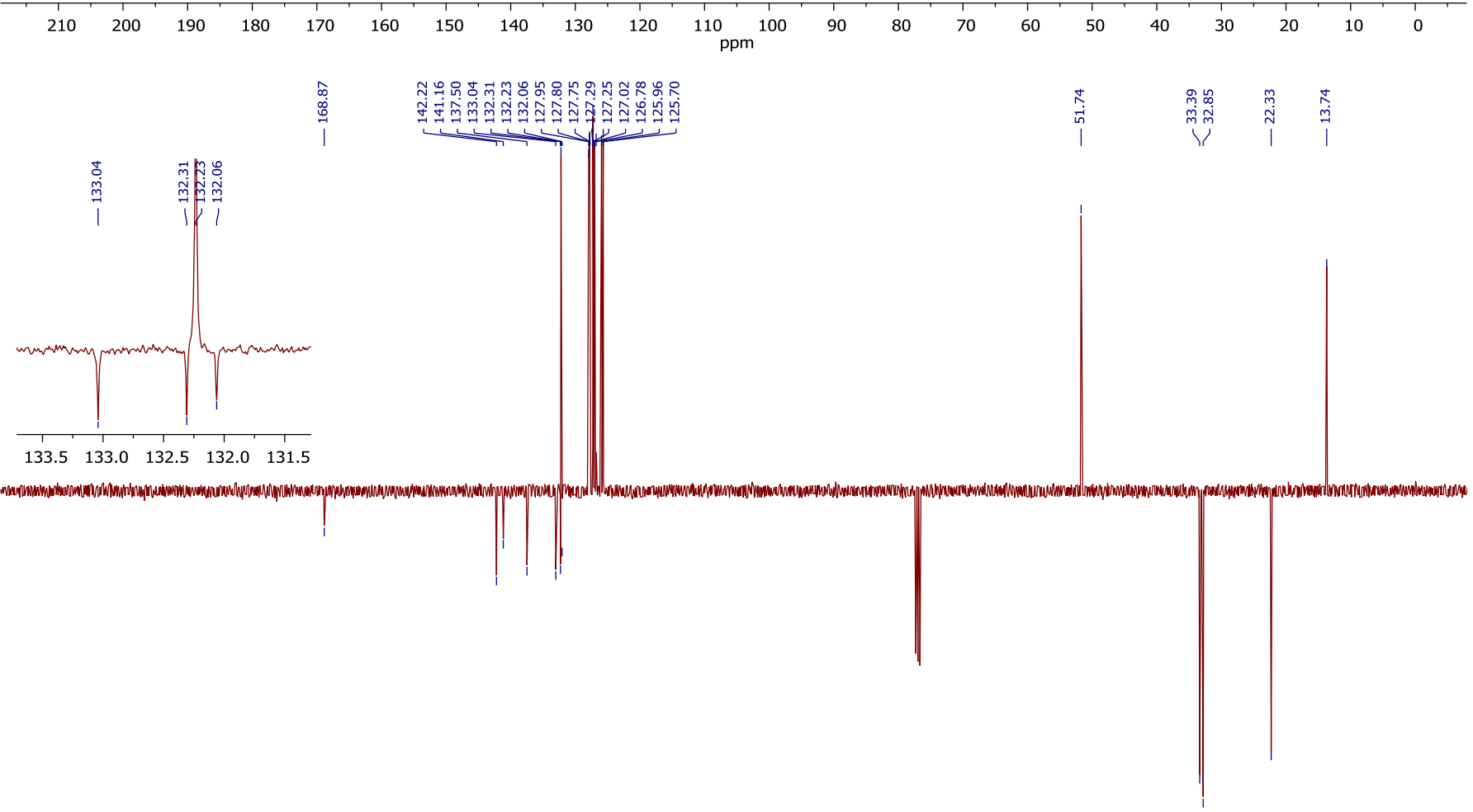

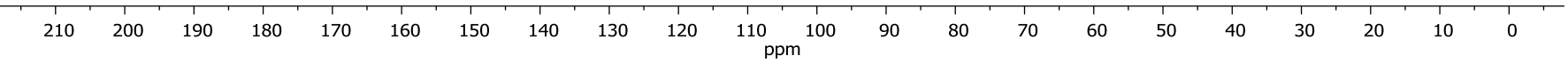




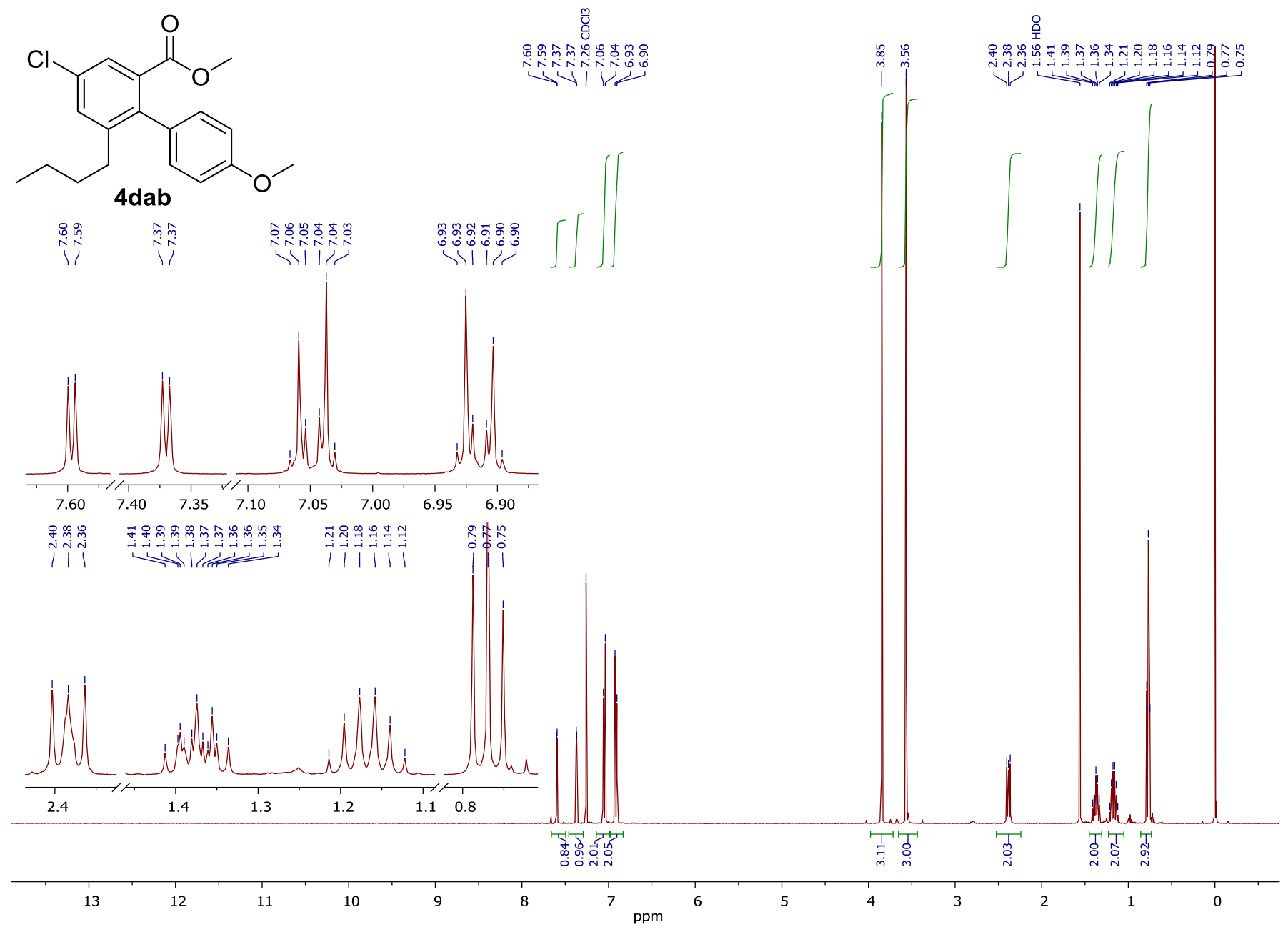



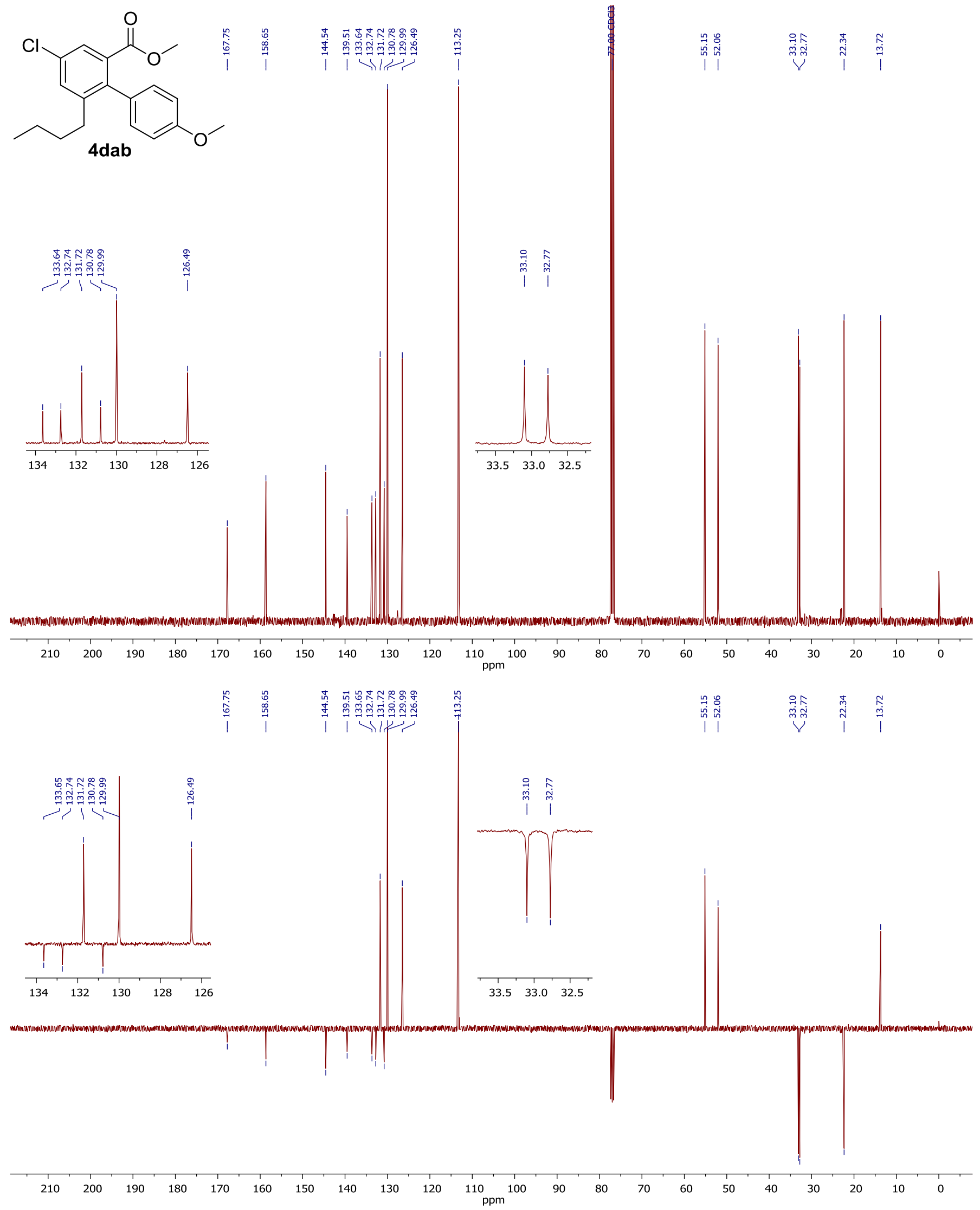
<smiles>CCCCc1cccc([N+](=O)[O-])c1-c1ccc(OC)cc1</smiles>

営

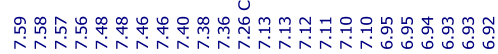

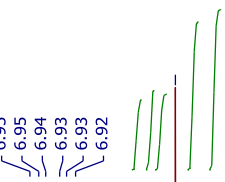

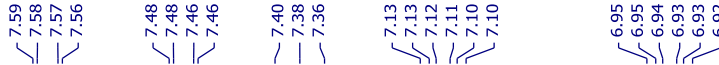

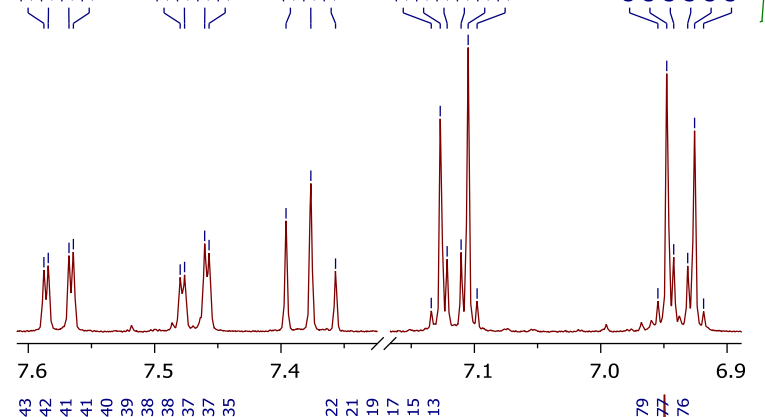

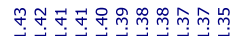

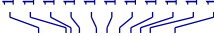

जितनें

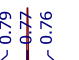

i金家

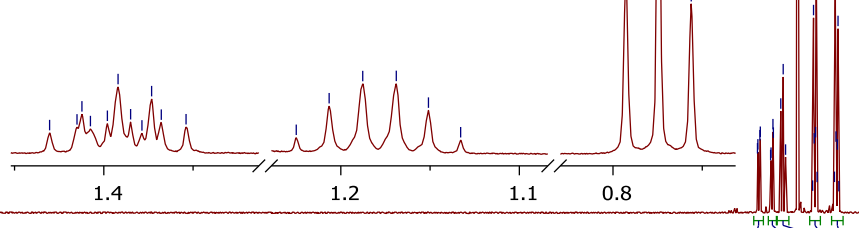

13

11

10

9

8

$7 \mathrm{ppm}$ 
<smiles>CCCCc1cccc([N+](=O)[O-])c1-c1ccc(OC)cc1</smiles>

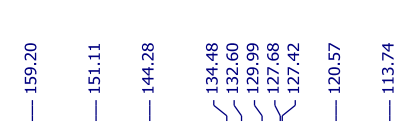

㖴
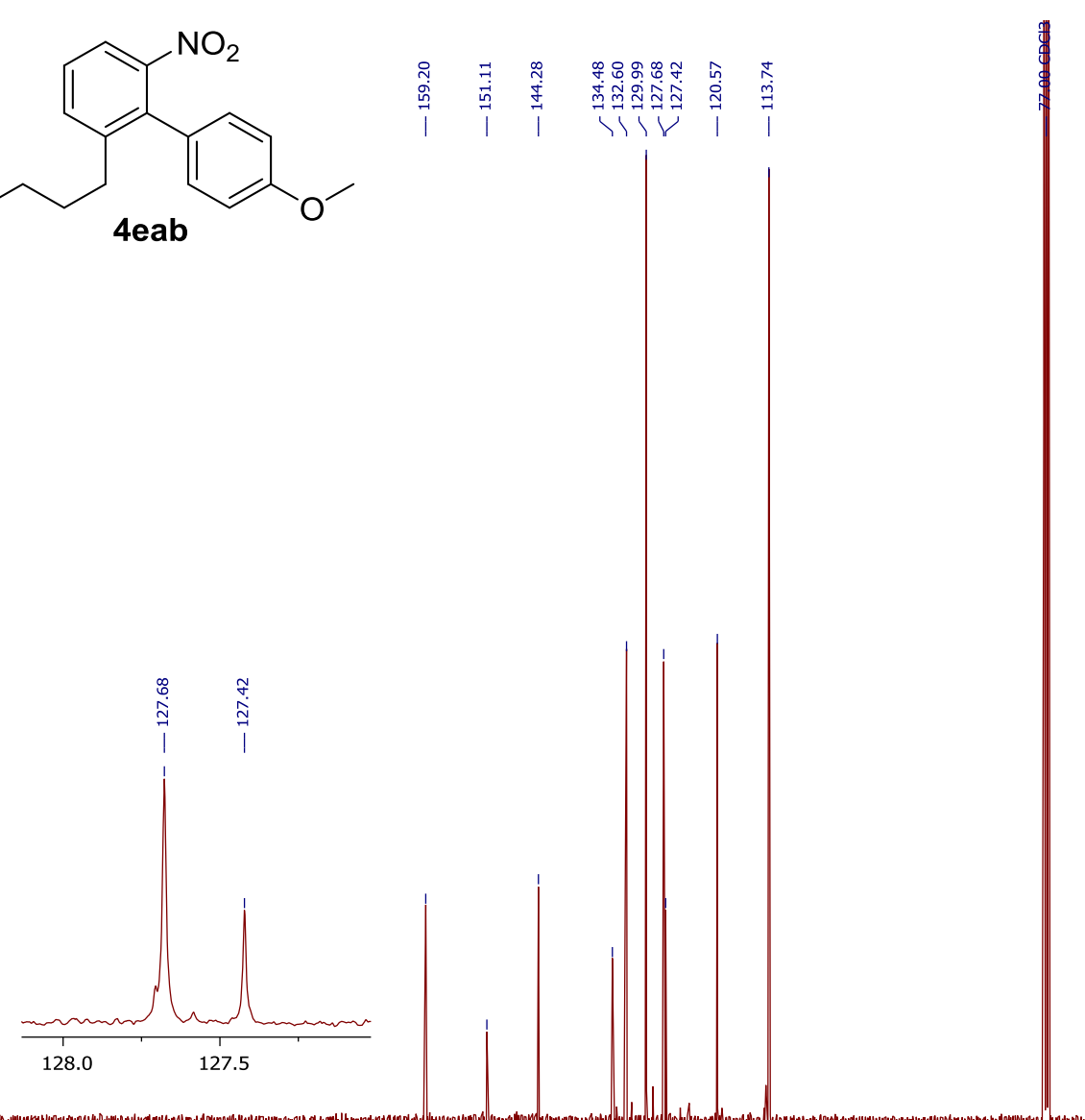

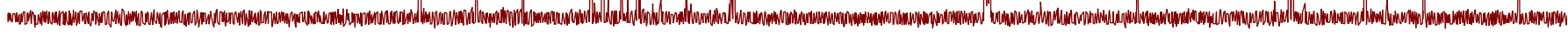
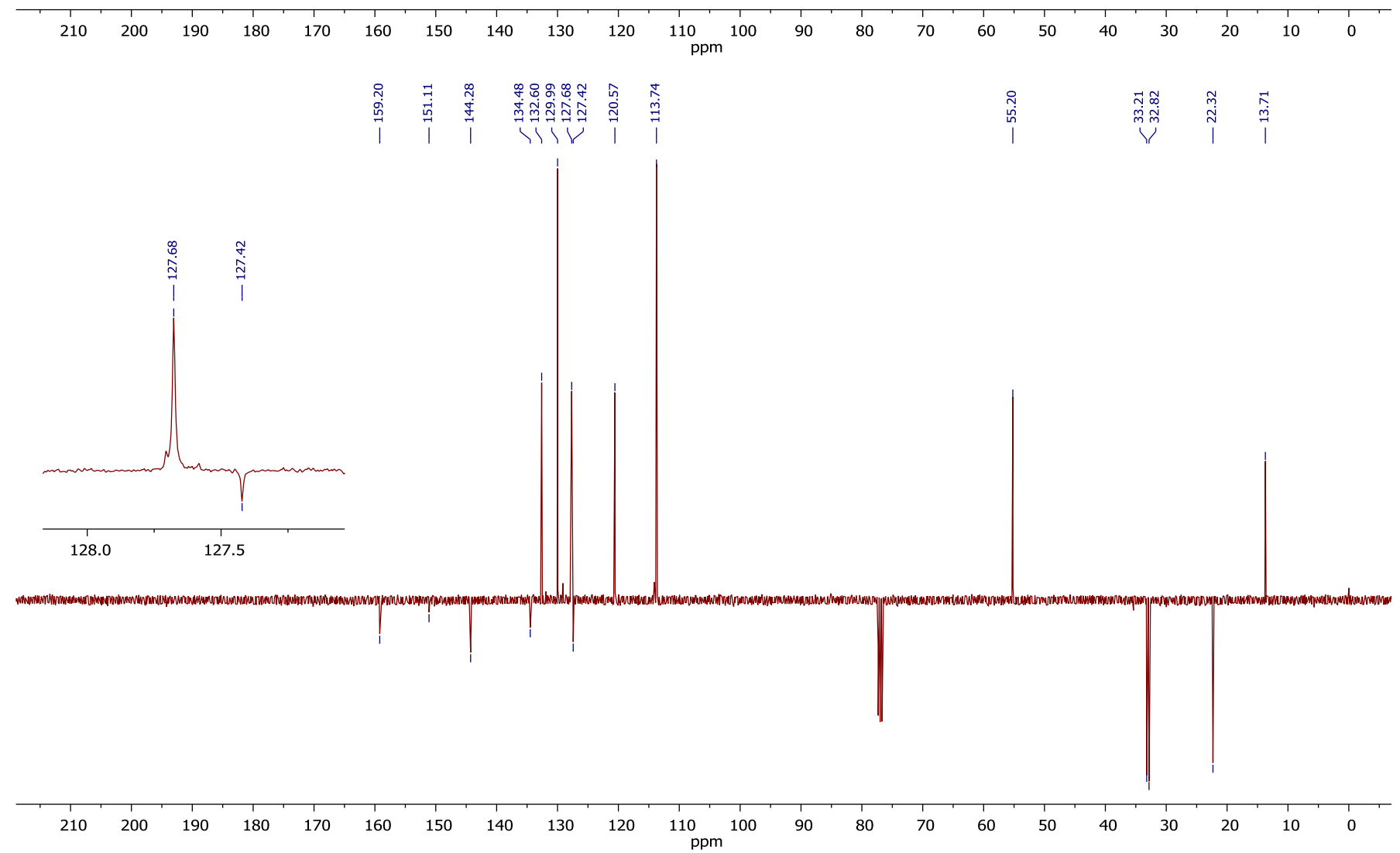


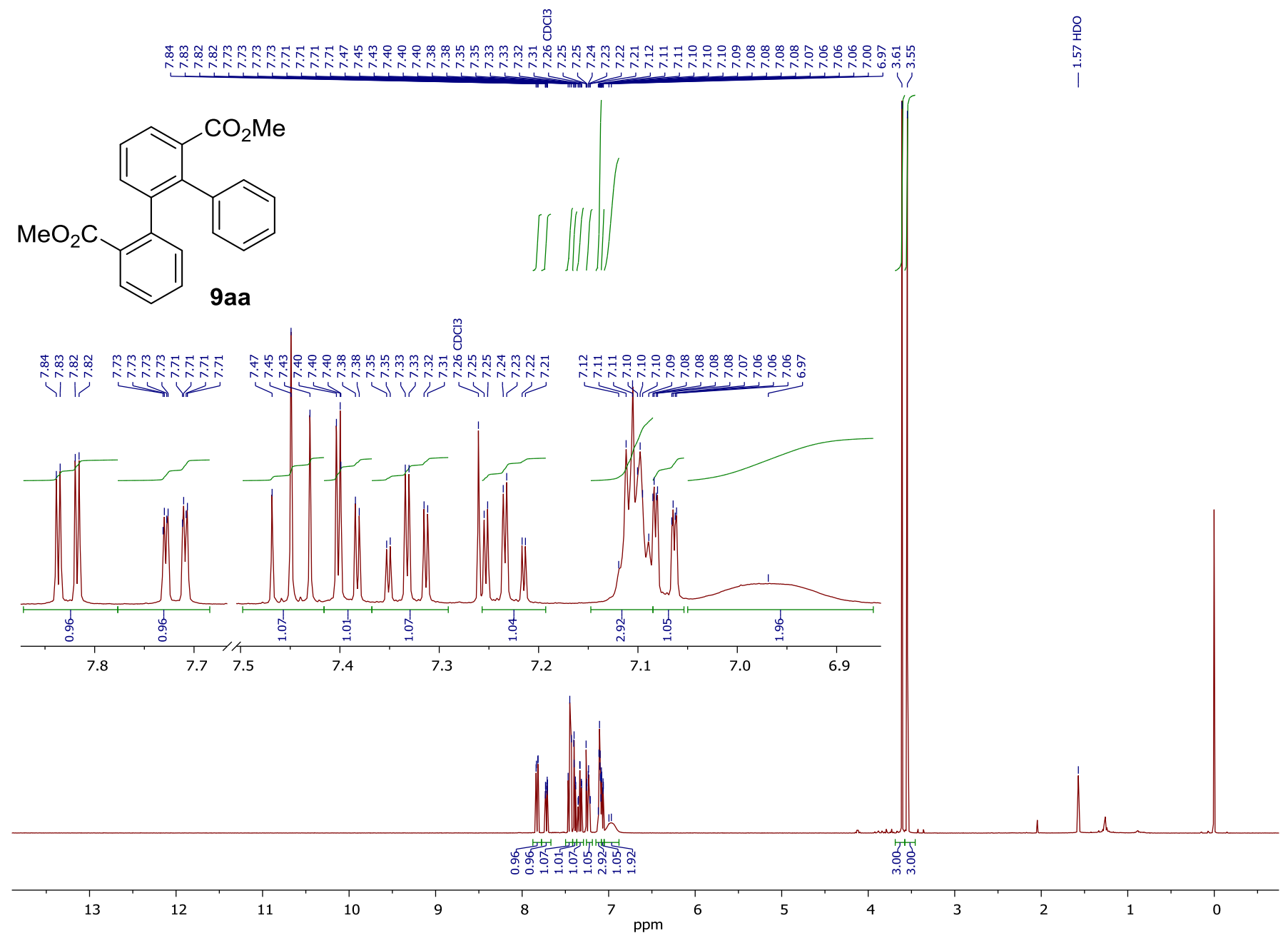



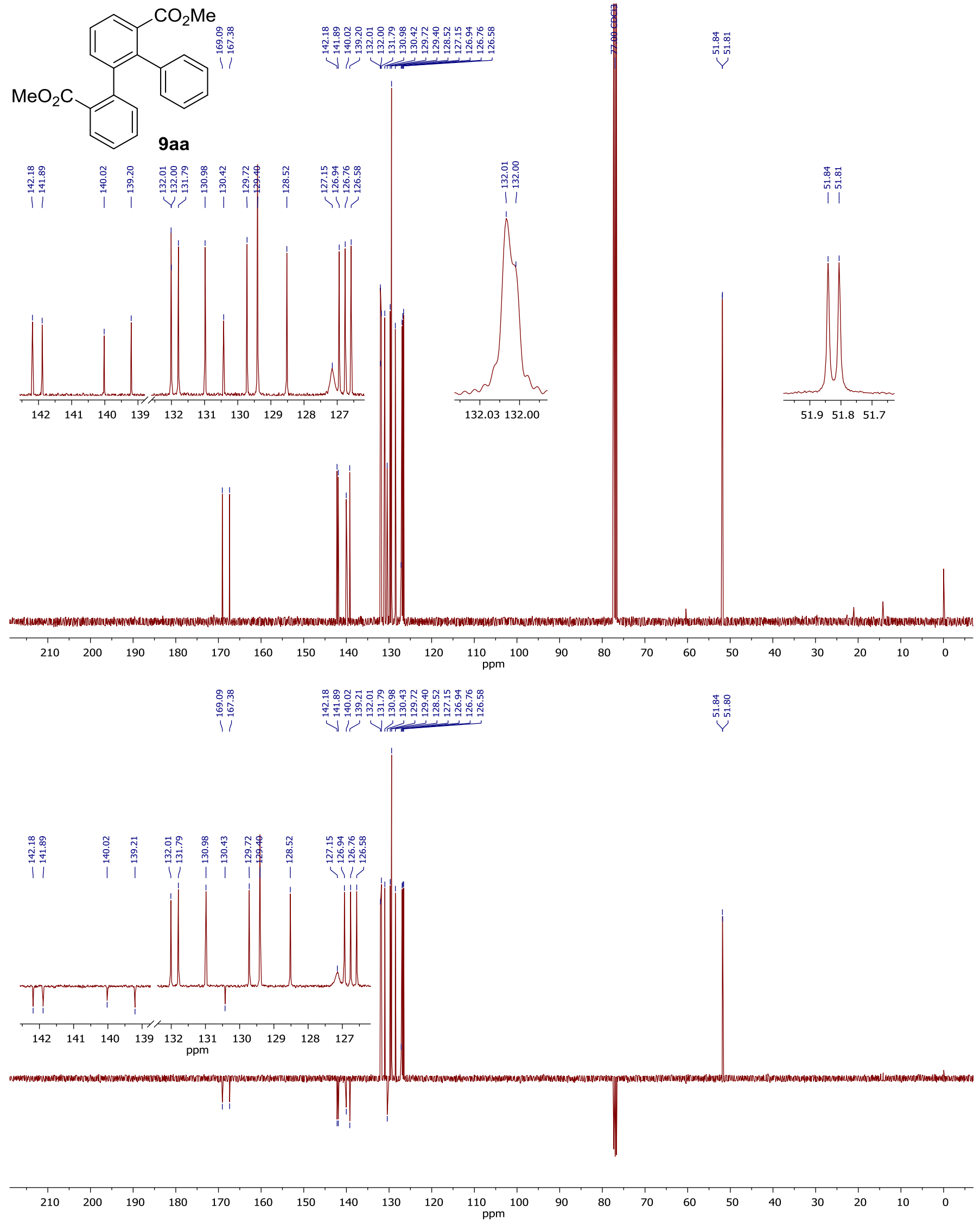
<smiles>CCCCc1cc(C(=O)OCC)cc(CCCC)c1-c1ccccc1</smiles>

10qaa

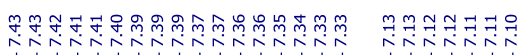

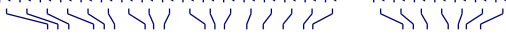
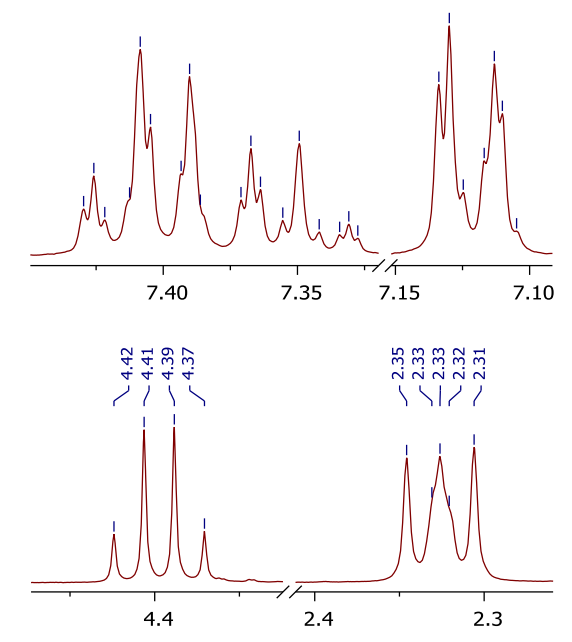

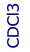

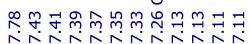

ivinisinin

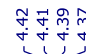

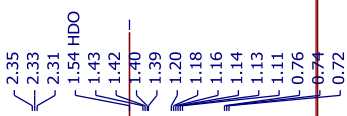

$\int d \mid$

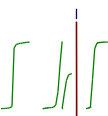

$\int$
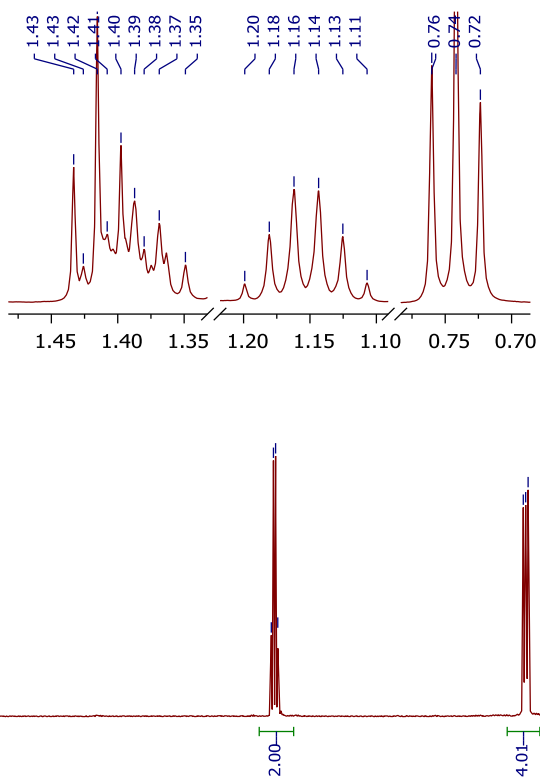


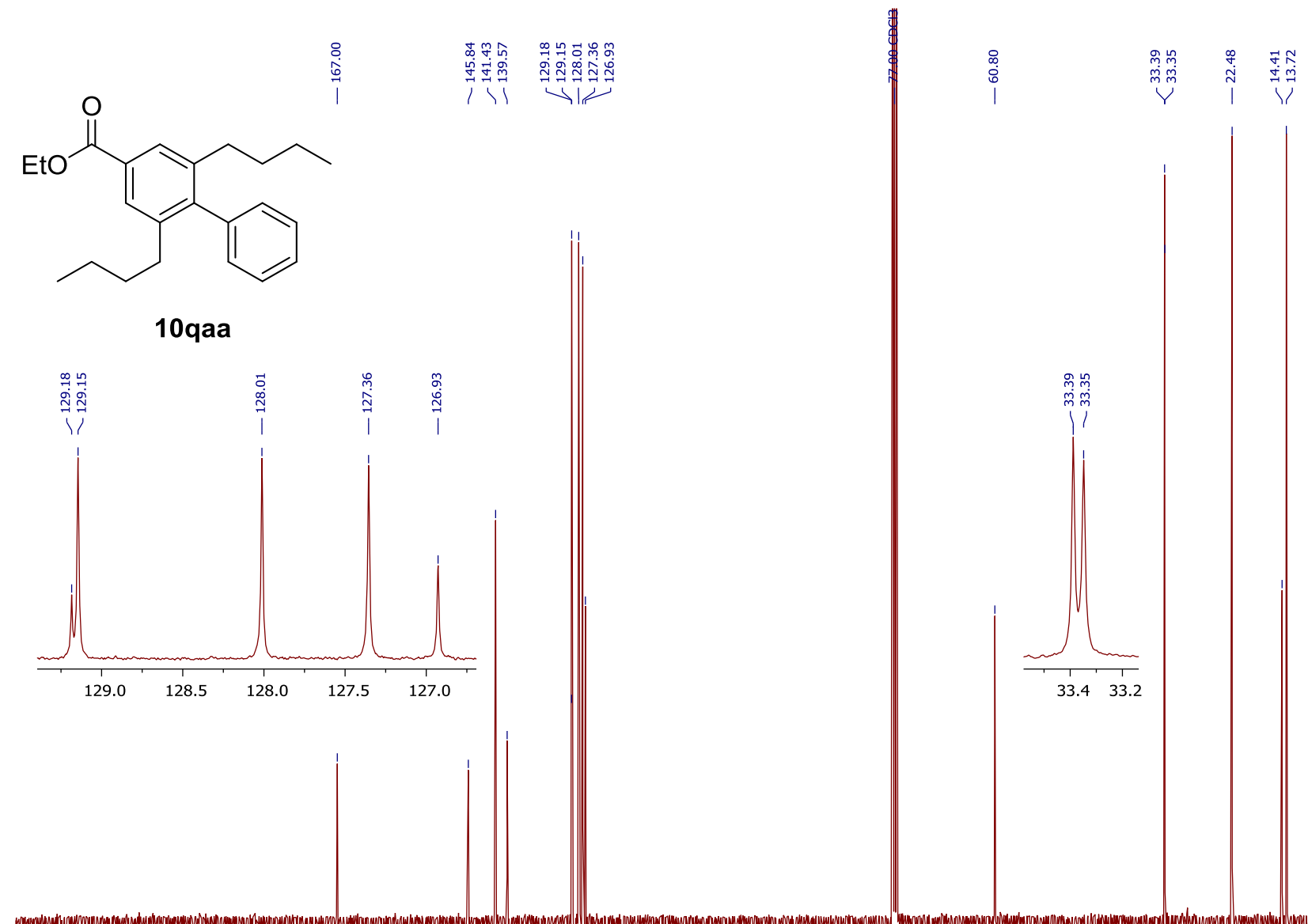

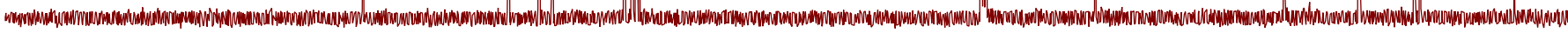
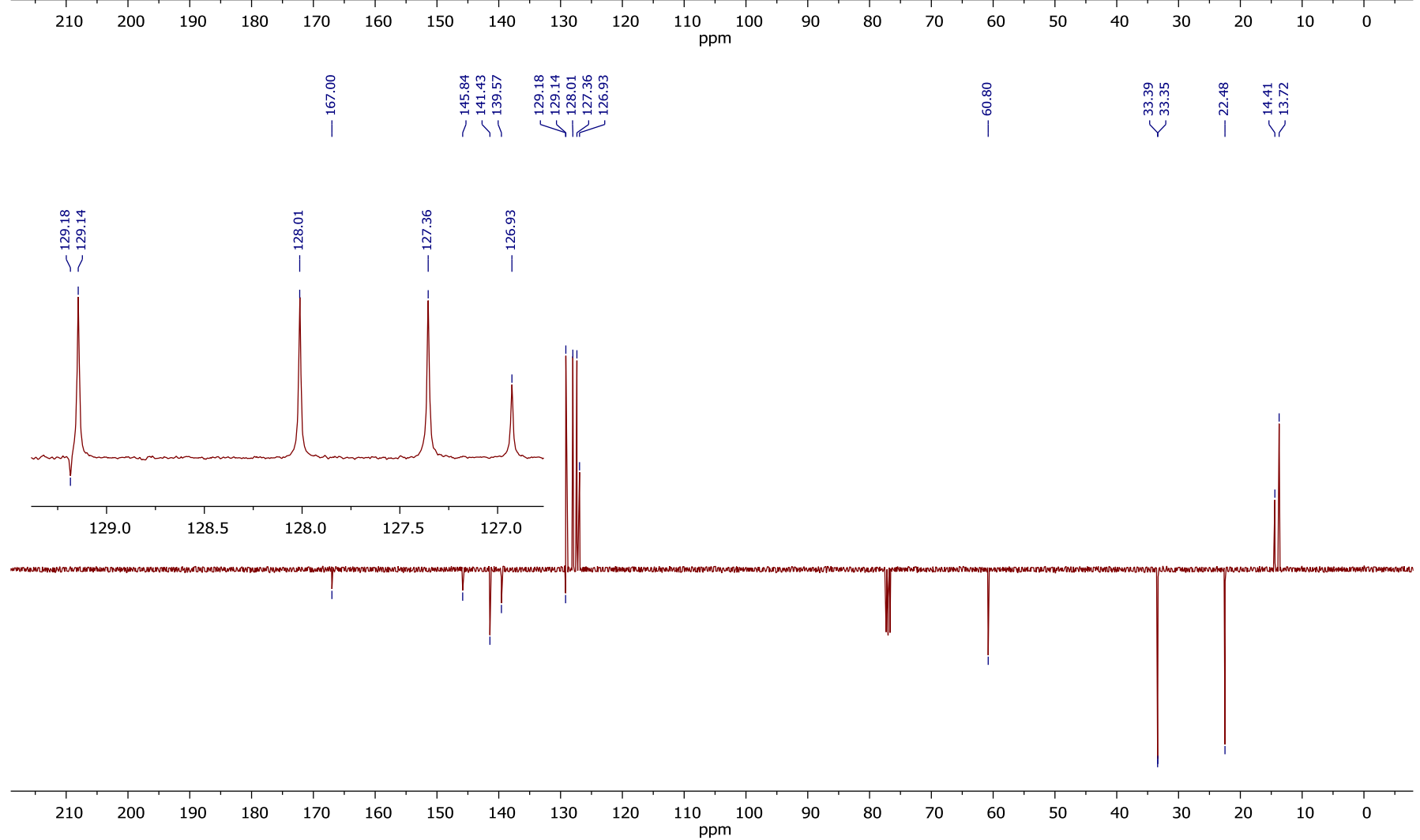